UCRI-Miz-110662-Pt.3

D293 005827

\title{
EQ3NR, A Computer Program for Geochemical Aqueous Speciation-Solubility Calculations: Theoretical Manual, User's Guide, and Related Documentation (Version 7.0)
}

Thomas J. Wolery 


\section{Preface}

This repon on the EQ3NR code is a revision of the first EQ3NR User's Guide (Wolery, 1983). It is one of a set of reports documenting version 7.0 (version 3245.1090 under the old numbering system) of the EQ3/6 software package. This set includes:

I. The EQ3/6 Package Overview and Installation Guide (Wolery 1992).

II. The EQPT User's Guide (Daveler and Wolery, 1992).

III. The EQ3NR Theoretical Manual and User`s Guide (this report).

IV. The EQ6 Theoretical Manual and User's Guide (Wolery and Daveler, 195,2).

EQ3NR, the subject of the present report, is the speciation-solubility code in the EQ3/6 incking. EQ6 is the reaction path code in EQ3/6. EQPT is the EQ3/6 data file preprocessor. The preseni report assumes that the reatder is familiar with the contents of the EQ3/6 Package Overview and Installation Guide and the EQPT User`s Guide.

The development of EQ3/6 has been supported by a number of programs concerned with alogic disposal of high level nuclear waste, including the Office of Nuclear Waste Isolation, the Salt Repository Project Office, the Waste Isolation Pilot Plant (through Sandia National Laborat $y$ ). the Nevada Nuclear Waste Storage Investigations, and the Yucca Mountain Site Characterizi." on Project. Documentation for the package is aimed at satisfying the reguirements of the U.S. Nuclear Regulatory Commission for sofiware used for this purpose (Silling, 1983).

The Litwence Livermore National Laboratory has not certified that EQ3/6 constitutes approved code for the conduct of quality affecting work for the Yucca Mountain Project.

No source codes or data files are reproduced in this report, nor are any computer media containing such items a paut of this report or any of the other reports documenting this version of EQ3/6. The software itself must be obtained as described below.

The examples presented in this series of reports correspond to version 7.0 of the software and the R10 set of supporting thermodynamic data files. As of the date of publication of this report, the most recent version of the software is version 7.1 (containing bug fixes, but no enhancements), and the most recent set of data files is R16.

Agencies of the United States Government and their contractors maly obtain copies of the softwatre and its documentation from:

Energy Science and Technology Software Center

P. O. Box 1020

Oak Ridge, TN 37831-1020

Telephone: (615) 576-2606 
Requests to obtain the software under a licensing agreement should be addressed to:

Technology Transfer Initiatives Program, L-795

Attn: Diana (Cookie) West

Lawrence Livermore National Laboratory

P.O. Box 808

Livermore, CA 94550

Telephone: $(510)$ 423-7678

Fax: $(510) 422-6416$

Secretary: (510) 422-6416

Comments and questions concerning EQ3/6 exclusive of the thermodynamic data base shouk' be addressed to the code custodian:

Thomas J. Wolery, L-219

Lawrence Livermore National Laboratory

P.O. Box 808

Livermore, CA 94550

E-mail: wolery1@1ln1.gov

Telephone: (510) $422-5789$

Fax: (510) 422-0208

Secretary: (510) 423-2970

Comments and questions which concern the EQ3/6 thermodynamic data base should be addressed to the data base custodian:

James W. Johnson, L-219

Lawrence Livermore National Laboratory

P.O. Box 808

Livermore, CA 94550

E-mail: johnson@s05.es.llnl.gov

Telephone: (510) 423-7352

Fax: (510) 422-0208

Secretary: (510) 423-2970 


\section{Contents}

Glossary of Symbols ..................................................................................................... v

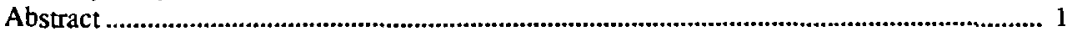

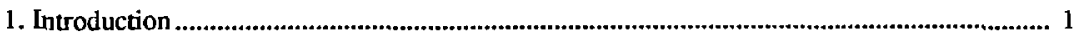

2. Speciation-Solubility Modeling of Aqueous Systems ............................................................. 9

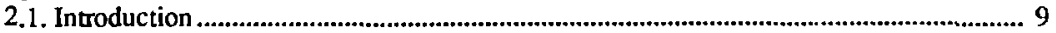

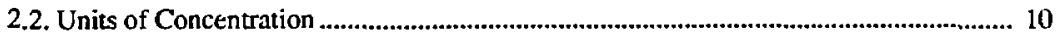

2.3. Input Constraints, Governing Equations, and Outputs................................................. 12

2.3.1. Overview .......................................................................................................... 12

2.3.2. Reference Formulation of the Aqueous Speciation Problem.............................. 13

2.3.3. Altemative Constraints....................................................................................... 15

2.3.4. $\mathrm{pH}$ in Brines: $\mathrm{pHCl}$ and Related Functions as Altemative Constraints .............. 19

2.3.5. The Carbonate System: Dealing with Alkalinity .................................................. 21

2.3.6. Redox Constraints ................................................................................................ 27

2.3.6.1. There is No "System" Eh in Most Real Systems ................................... 27

2.3.6.2. Background: Redox Couples and Half-Reactions................................ 28

2.3.6.3. Background: $E h, p e, A h$, and Equilibrium Oxygen Fugacity............... 29

2.3.6.4. Redox Options: Testing versus Assuming Equilibrium........................ 32

2.3.7. Measures of Mineral Saturation................................................................... 33

2.4. Use and Misuse of Speciation-Solubility Codes ............................................................. 34

3. Activity Coefficients of Aqueous Species ............................................................................ 36

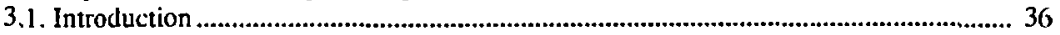

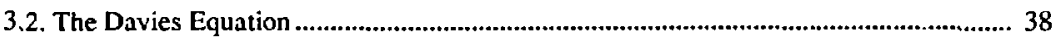

3.3. The B-dot Equation ................................................................................................. 39

3.4. Scaling of Individual Ionic Activity Coefficients: $\mathrm{pH}$ Scales ...................................... 42

3.5. Pitzer`s Equations..................................................................................................................... 44

3.5.1. Introduction ....................................................................................................... 44

3.5.2. Solutions of Electrolytes ............................................................................. 46

3.5.3. Solutions of Electrolytes and Nonelectrolytes ..................................................... 54

3.5.4. Temperature and Pressure Dependence ............................................................... 61

3.5.5. Practical Aspects ............................................................................................... 62

3.5.6. Pitzer's Equations in EQ3/6: Current Status ....................................................63

4. Activity Coefficients of Solid Solution Components................................................................ 65

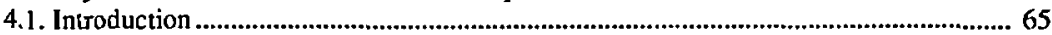

4.2. ldeal Solution, with One Optional Site-Mixing Parameter............................................ 67

4.3. Third-Order Maclaurin Model for a Binary Solution ..................................................... 68

4.4. Regular Solution Model for a Binary Solution ......................................................... 68

4.5. Cubic Maclaurin Model for a Binary Solution ................................................................. 69

4.6. Guggenheim Polynomial Model for a Binary Solution …............................................ 69

4.7. Regular Solution Model for a Ternary Solution ................................................... 70

5. Basis Species: Key Concepts ....................................................................................... 71

5.1. Basis Species

5.2. Organization and Treatment of Basis Species ............................................................. 74

5.3. Stoichiometric Conversions of Analytical Data.......................................................... 77

6. The EQ3NR Input File: Setting up the Problem ……........................................................ 78

6.1. Input File Characteristics and Contents ................................................................. 78 


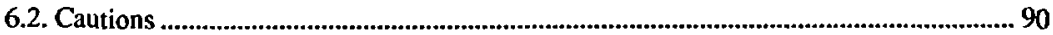

7. Sample Problems: Inputs and Outputs................................................................................. 92

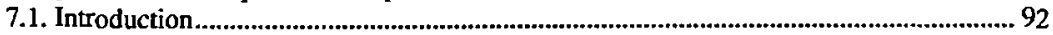

7.2. Sea Water Test Case, with Major Cations and Anions Only ........................................... 92

7.3. The Sea Water Test Case, Using Pitzer's Equations ....................................................... 103

7.4. Using Mineral Solubility Constraints: An Example ........................................................ 110

7.5. Calculating the Composition of a Custom pH Buffer, An Example ............................. 118

7.6. Computing Oxygen Fugacity from Mineral Equilibria: An Example ........................... 124

7.7. Computing Eh from a Redox Couple: An Example ........................................................ 130

7.8. The Dead Sea Brine Test Case............................................................................................... 138

7.9. Using $\mathrm{pHCl}$ as an Input: An Example ........................................................................ 146

8. The EQ3NR to EQ6 Connection: The Pickup File.................................................................. 153

9. Solving the Governing Equations ..................................................................................... 156

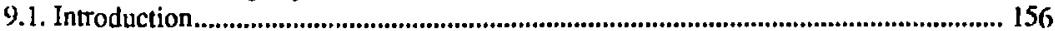

9.2. The Set of Master Iteration Variables............................................................................. 156

9.3. Expanding the System from the Set of Master Iteration Variables ................................ 358

9.4. Beginning the Process: Computing Starting Values....................................................... 159

9.5. Methods to Aid Convergence ............................................................................................ 160

9.6. The Pre-Newton-Raphson Optimization Algorithm................................................... 163

9.7. The Newton-Raphson Method................................................................................ 171

9.8. Derivation of Residual Functions and the Jacobian Matrix...................................... 173

9.8.1. Mass Balance ..................................................................................................... 173

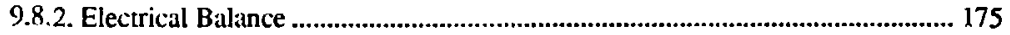

9.8.3. The Mole Fraction Of Water........................................................................ 176

9.8.4. Specified Free Concentration............................................................................. 178

9.8.5. Specified Thermodynamic Activity ................................................................ 178

9.8.6. Log Activity Combination ............................................................................ 178

9.8.7. Mean Log Activity............................................................................... 179

9.8.8. Equilibrium With A Pure Mineral .............................................................. 180

9.8.9. Equilibrium With A Solid Solution End-member Component.......................... 180

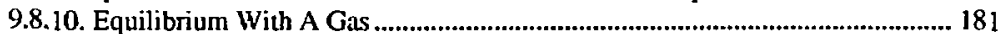

9.8.11. Concentration Fixed By Internal Equilibrium ............................................... 182

9.8.12. Specified Log Oxygen Fugacity ...................................................................... 183

9.8.13. Specified Eh ........................................................................................................ 183

9.8.14. Oxygen Fugacity Fixed by An Aqueous Redox Couple.................................... 184

9.9. Methods for Crash Diagnostics.................................................................................... 185

10. Code Architecture and Flow of Execution................................................................. 188

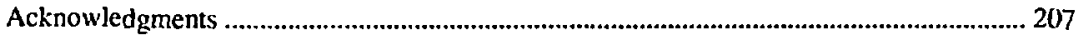

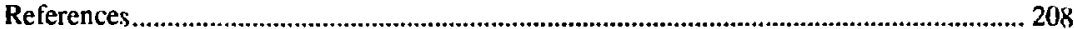

Appendix A: Glossary of Mijor Variables in EQ3NR ....................................................216

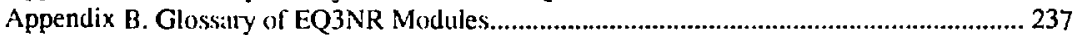

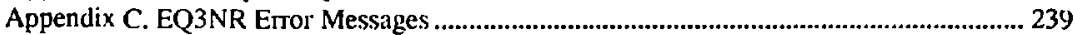

Appendix D. Notes on Known Bugs and Such............................................................... 246 


\section{Glossary of Symbols}

$a, a^{\prime} \quad$ Symbols used to represent cations in the notation of Harvic, Moller, and Weare (1984); see also $X, X^{\prime}$.

$a_{i} \quad$ Themodynamic activity of the $j$-th agueous solute species.

$a_{w} \quad$ Thermodynanic activity of water.

$a_{ \pm M X} \quad$ Mean activity of neutral electrolytc $M X ; \log a_{ \pm, M X}=\frac{v_{M} \log a_{M}+v_{X} \log a_{X}}{v_{M X}}$.

$a_{\text {or }} \quad$ Themodlynamic activity of the $\sigma$-th component of the $\psi-$ th solid solution phase.

a Gencralized hard core diancter or "ion size" in aqueous solution.

$d_{l} \quad$ Hard corc dianeter $\alpha$ "size" of the $i-t h$ aqueous solute species.

A Affinity.

$A_{j} \quad$ Themodynamic alTjully of the $j$-th rcaction. The forward direction is implicd, which in $E Q 3 / 6$ is taken to be that in which the associated species is destroyed, for example by dissolution or dissociation.

$A_{+j} \quad$ Themodynamic affinity of the $j$-th reaction (forward direction).

$A_{-j} \quad$ Themodynamic affinity of the $j$-th reaction (reverse direction).

Ah Themodynanic aftinity (per electron) of a redox couple with respect to the standard hydrogen electrode; $A h=F E h$.

$A_{k} \quad$ Titration alkalinity, in units of equivalents per kjlogram of solvent water or equivalents/L.

$A_{\gamma, e} \quad$ Debye-Hückel $A$ parameter used in writing expressions for $\ln \boldsymbol{y}_{\text {- }}$.

$A_{\gamma_{1} 10} \quad$ Debye-Hückel $A$ parameter used in writing expressions for $\log _{\ell 0} \gamma_{i} ; A_{\gamma_{\ell}, 10}=2.303 A_{\gamma_{, e}}$

$A_{Q} \quad$ Debye-Hückel $A$ parameter used in writing expressions for $\ln a_{m \text {. }}$.

$A_{t}^{*} \quad$ Titration altalinity, mg/L of equivalent $\mathrm{CaCO}_{3} ;$ also denoted as $T$.

$A_{t, H \mathrm{CO}_{3}^{-}}^{*} \quad$ Titration altalinity, $\mathrm{mg} / \mathrm{L}$ of equivalent $\mathrm{HCO}_{3}^{-}$.

$A_{\text {IfCO; }}^{*} \quad$ Bicarlsonatc alkalinity, $\mathrm{mg} / \mathrm{L}$ of equivalent $\mathrm{CaCO}_{3}$.

$A_{\mathrm{CO}_{3}^{2 *}}^{*} \quad$ Carhonate alkalinity, $\mathrm{ng} / \mathrm{L}$ of equivalent $\mathrm{CaCO}_{3}$

$A_{\text {oH }}^{*} \quad$ Hydroxide alkalinity, mg/L. of tequivalem $\mathrm{C}_{\mathrm{C}} \mathrm{CO}_{3}$.

$b_{s r} \quad$ Stoichionetric reaction coefficient, the number of moles of the $s$-th aqueous sjecics appearing in the $r$-th aqueous reaction; it is negative for reactants and positive for products. 
$b_{s \phi} \quad$ Stoichionetric reaction coefficient, the number of moles of the $s$-th aqueous species appearing in the reaction for the dissolution of the $\phi-$ th pure mineral; it is negative for reactants and positive for products.

$b_{s g} \quad$ Stoichiometric reaction coefficient, the number of moles of the s-th aqueous species appearing in the reaction for the dissolution of the $\boldsymbol{g}$-th gas species; it is negative for reactants and positive for products.

$b$

$B_{\gamma}$

$B_{A f X}(I)$

$B_{M X}^{\prime}(I)$

$B_{M X}^{\phi}(I)$

$c, c^{\prime}$

$C_{i, \text { molar }}$

$C_{i, m g / L}$

$C_{i, m g / k g}$

$C_{T S, m g / k g}$.

$C_{T S, m g} L$

$C_{\text {IIX }}^{0}$

$C_{M X}$

$\varepsilon$

$\varepsilon_{T}$

$e^{-}$

$E_{j}$

$l_{j}^{0}$

Eh

A parameter theorelically equivalent to the product $\& B_{y}$ ankl appcaring in Pitzer's equations with an fixed value of 1.2

Debyc-Hückcl $B$ parameter used in writing expressions for $\ln \gamma_{j}$ or $\log _{j 0} \gamma_{i}$.

Observable second order interaction coeficient for neulsal electrolyte $M X$ ( $M=$ cation, $X=$ antion); a function of the ionic strength.

The derivative of $B_{A I X}(I)$ with respect to ionic sIrength.

The conjpound function $B_{M X}(I)+I B^{\prime}{ }_{M X}(l)$.

Symbols used to represent cations in the notation of Harvic, Moller, and Weare (1984); see also $M$. $M$.

Concentration of the $i$-th solute species in molarity (moles/L).

Coucentration of the $i$-th solute species in $\mathrm{mg} / \mathrm{L}$.

Concentration of the $i$-th solute species in me/tg solution.

Total dissolved solutes in $\mathrm{mg} / \mathrm{kg}$ solution.

Total dissolved solutes in $\mathbf{m g} / \mathbf{L}$.

Thind order interaction coefficient for neutral elcctrolyte $M X$.

The quantity $\frac{C_{M X}^{\circ}}{2 \sqrt{z_{M}{ }^{2} X}}$.

Subscript indexing a chemical clenxut.

Total number of chemical elements in a system.

The electron. In common themodynamic fomalism, this is ustadly a hypothelical species, not a real onc.

Electrical potentjal of the $j$-1h nedox couple, volts.

Standard state electrical potential of the $j$-th redox couplc, volts.

Redox potentiai, volts. Theoretical equilibritun electrical potential of a redox couple:

$E h=\frac{2.303 R T}{4 V}\left(\log \int_{O_{2}}-4 p H-2 \log a_{1 v}-\log K_{E l_{1}}\right)$, where $\int_{O_{2}}$ is understood to lx due hypothetical equilibrium oxygen fugacity in aţucous solution. 
The quantity $f(I) / 2$.

Oxygen fugacity.

F

g

Br

$g(x)$

$g^{\prime}(x)$

$\mathrm{G}^{E X}$

$H_{s r}$

$H_{z}$

I

$I^{P}$ P

$J(x)$

$f(x)$

$J_{i j}$

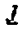

K

$K_{E h}$

$K_{\text {w }}$

$m_{i}$

$m_{T, i}$

$M, M^{\prime}$

$M_{j}$
(1) The Faraday constant, $23062.3 \mathrm{cal} / \mathrm{cquiv}$-volt; (2) Compound electrostatic function used by Harvie, Moller, and Weare (1984) to write Pitzer's equations (sce Chapter 3).

Subscript denoting a gas species.

Total number of gas species in a system.

A function used to describe the ionic strength dependence of the second order interaction coefficient in Pilzer's equations.

The derivative of $g(x)$ with respect to $x$.

Excess Gibbs energy, as of a solution.

The factor $\frac{m_{s^{\prime \prime}} u_{s^{\prime \prime} r}}{b_{s^{\prime \prime} r}}$, where $s^{\prime \prime}$ denotes the dipendent aqueous species which is associated with and destroyed by the $r$-th aqueous reaction.

The factor $\frac{m_{s^{n}} Z_{s^{n}}}{b_{s^{*}}}$ (analogous to $H_{s r}$ ).

Ionic strength.

Ion activity product; $\sec Q$.

A function used to describe the bigher order electrical interactions term in Pitzer's equations.

The derivative of $J(x)$ with respect to $x$.

An element of the Jacobian matrix $\left(\frac{\partial \alpha_{i}}{\partial z_{j}}\right)$.

The Jacobian matrix.

Tbemodynamic equilibrium constant.

Themodynanic equilibrium constant for the hali-reaction $2 \mathrm{H}_{2} \mathrm{O}_{(\mathrm{l})}=\mathrm{O}_{2(\mathrm{~B})}+4 \mathrm{H}^{-1}+4 e^{-}$.

Equilitrium constant for the reaction $\mathrm{H}_{2} \mathrm{O}_{(1)}=\mathrm{H}^{+}+\mathrm{OH}^{-}$

Molal concentration of the $i$-th aqueous solute specics (no contributions from dependent species).

Total molal concentration of die $i-$ hh aqueous species (includes contributious from dependent species).

Symbols denoting cutions (see also $c, c^{\prime}$ ).

Molecular weight of the $i$-lin substance, grans per mole; c.g. $M_{w}$ is the molecular weight of water. 


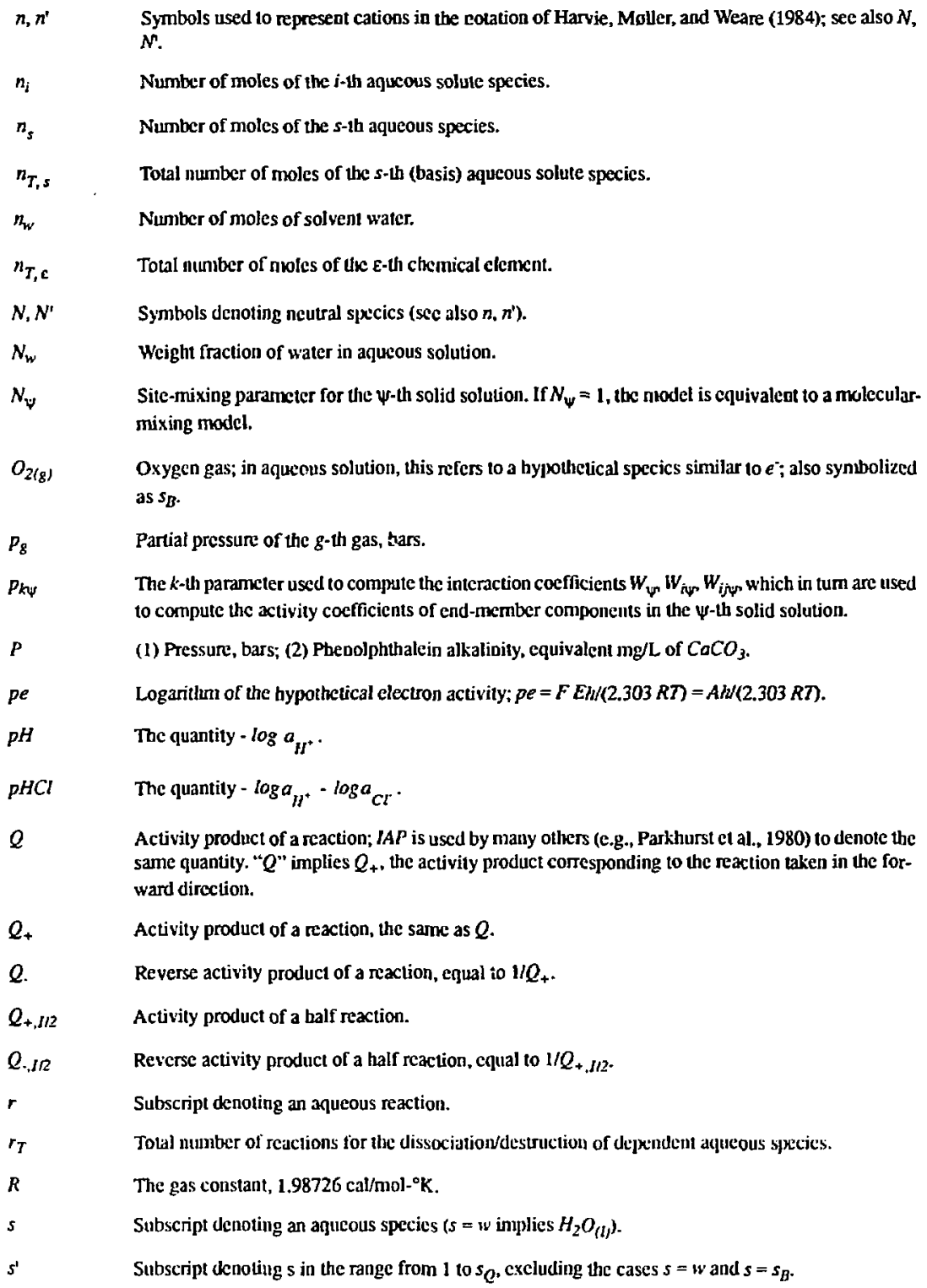
same quantity. " $Q$ " implies $Q_{+}$, the activity product corresponding to the reaction taken in the forward direction.

$Q_{+} \quad$ Activity product of a maction, the same as $Q$.

$Q$ Reverse activity product of a reaction, equal to $1 / Q_{+}$.

$Q_{+, J 2} \quad$ Activity product of a half reaction.

$Q ., I I Z \quad$ Reverse activity product of a half reaction, cqual to $1 / Q_{+, J / 2}$. 


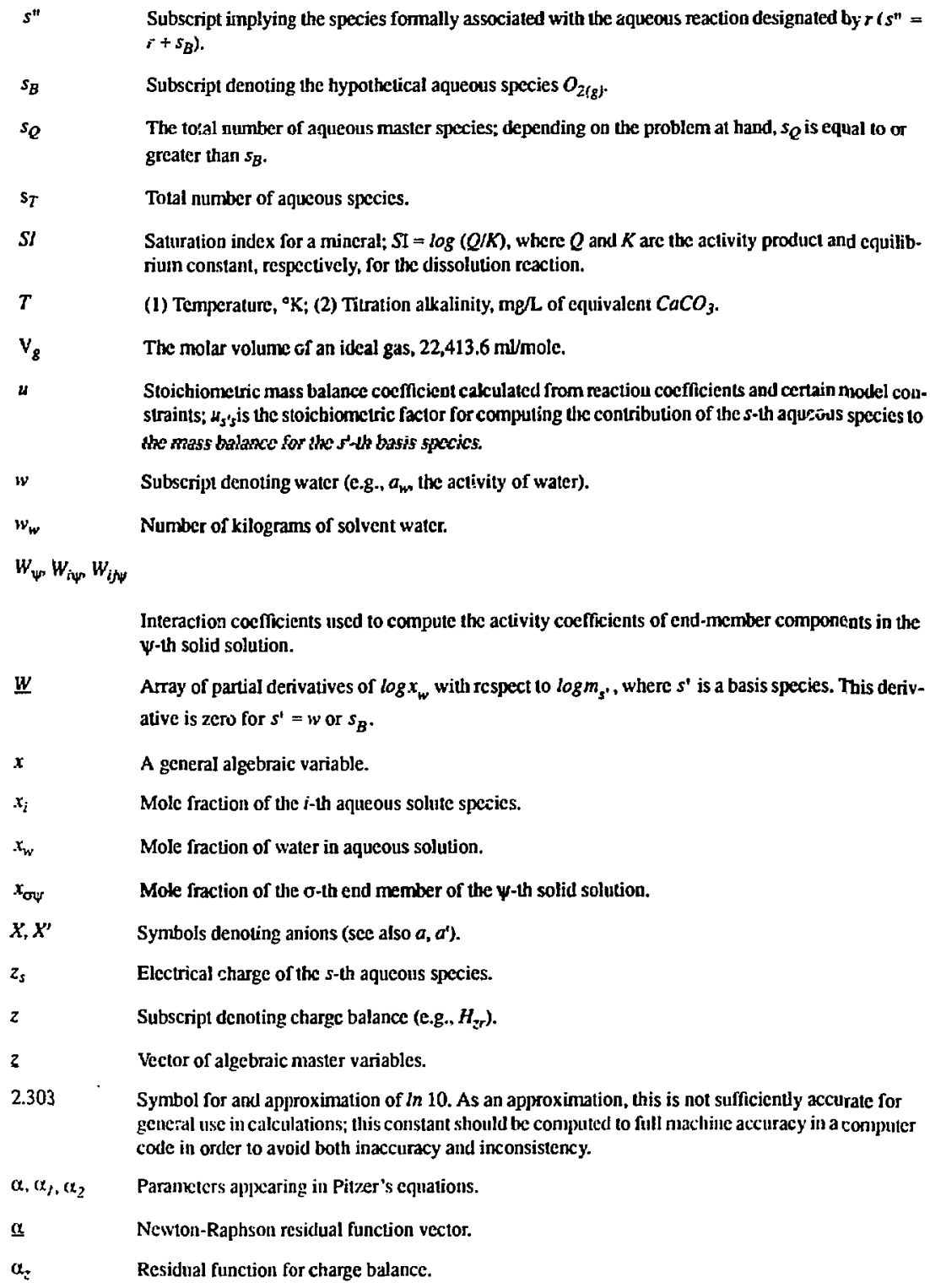

$x_{\sigma \psi} \quad$ Mote fraction of the $\sigma$-th end member of the $\boldsymbol{\psi}$-th solid solution.

$X, X^{\prime} \quad$ Symbois denoling anions (see also $a, a^{\prime}$ ).

$z_{s} \quad$ Electrical charge of the $s$-th aqueons species.

Subscript denoting the hypothetical aqueous species $O_{2(g)}$.

The total number of aqueous master species; depending on the problem at hand, $s_{Q}$ is equal to or greater than $s_{B}$.

Total number of aqueous species.

Saturation index for a mineral; $S I=\log (Q / K)$, where $Q$ and $K$ are the activity product and equilibrium constant, respectively, for the dissolution reaction.

(1) Temperature, ${ }^{\circ} \mathrm{K}$; (2) Titration alkalinity, me/L of equivalem $\mathrm{CaCO}_{3}$.

The molar volume of an ideal gas, $22,413.6 \mathrm{mLmole}$.

Stoichiometric mass balance coefficient calculated from neaction coefficients and certain model coustraints; $\mu_{s} s_{s}$ is the stoichiometric factor for computing the contribution of the $s$-th aquesins species to the mass balane for the s-lh basis species.

Subscripl denoting water (c.g., $a_{m}$ the activity of water).

Number of kilograms of solvent water.

Interaction coefficients used to compute the activity coefficients of end-member components in the $\Psi$-th solid solution.

Array of partial derivatives of $\log x_{W}$ with respect $10 \log m_{s^{\prime}}$, where $s^{\prime}$ is a basis species. This derivative is zero for $s^{\prime}=w$ or $s_{B}$.

A general algebraic variable.

Mole fraction of the $i$-th aqueous solute species.

Mole fraction of water in aqueous solution.

Subscript denoting charge balance (e.g., $H_{z r}$ ).

Vector of algebraic master variables.

2.303 Symbol for and approximation of $\ln 10$. As an approximation, this is not sufficiently accurate for general use in calculations; this constant should be conputed to full macline accuracy in a computer code in order to avoid both inaccuracy and inconsistency.

Paranctcrs appearing in Pitzer's cquations.

Newton-Raphson residual function vector.

Residual function for charge balance. 


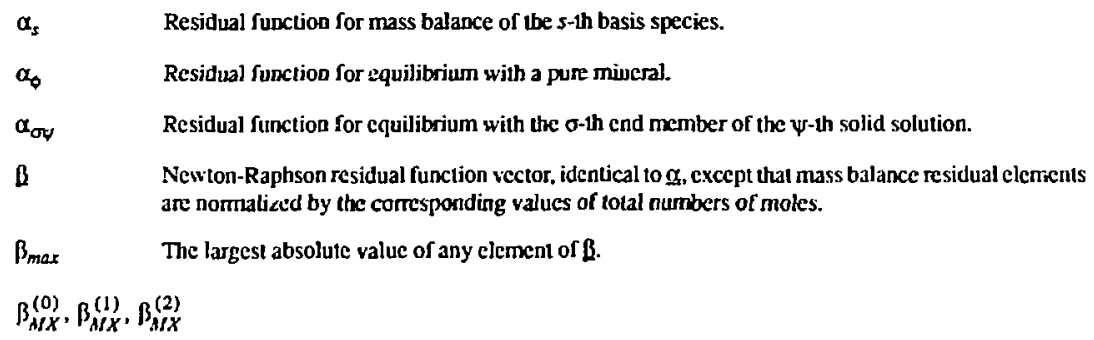

Observable second order interaction coefficient paraneters for weutral electrolyle $M X$.

$y_{i} \quad$ Molal aclivity cocficient of the $i$-th aqueous solute species.

$y_{T, 1} \quad$ Stoichiometric molal acliv:'; cocfficient of the $i$-th aqueous solute specics; generally sefined only for simple ions.

$y_{ \pm}, \mathrm{ME} \quad$ Mean molal activity coefficient of aqueous neutral clectrolyte $M X$.

$\gamma_{T, \pm}$ AE Stoichiometric mean molal activity coefficient of agueous neutral clectrolyte $M X$.

§. Newton-Raphson correction tem vector.

$\delta_{\max } \quad$ The largest absolutc value of any element of $\delta$.

$\delta_{\text {conv }} \quad$ Convergence function.

$\delta^{\prime} \quad$ Under-sclaxalion parameter.

$\Delta G_{f, i} \quad$ Gibbs energy of fomation of the $i$-th ctemical species.

$\Delta G_{f_{i} i}^{o} \quad$ Stankard state Gibbs energy of formation of the $i$-th chemieal species.

$\Delta G_{r} \quad$ Gibbs energy of reaction of the $r$-th reaction.

$\Delta G_{r}^{o} \quad$ Standard state Gibbs energy of reaction of the $r$-th reaction.

$\varepsilon \quad$ Subscript denoting a chemical clement.

$E_{T} \quad$ Total number of chemical elements in a system.

$\zeta_{\text {NMIX }}$ Observable third order interaction coefficient for neutral species $\mathrm{N}$, cation $\mathrm{M}$, and anion X.

$\theta_{M M}(l)$ Observable second order interaction coefficient for mixtures of neutral electrolytes $M X$ and $M X^{\prime}$; independent of the identity of $X$ and a function of the jonic strength.

$\theta_{\text {MM }}(I) \quad$ The derivative of $\theta_{M}(l)$ with respect to ionic strength.

$E_{\theta_{M / M}}(I) \quad$ The electrostatic part of $\theta_{M M}(I)$.

$s_{\theta_{M M^{\prime}}} \quad$ The short-range pan of $\theta_{M M^{\prime}}(l)$; treated as a coisstant. 
$\lambda_{i j}(I) \quad$ Second-order interaction coefficient for the $i$-1h and $j$-th aqueous solute species; in general, this is a function of the ionic strength.

$\lambda_{i j}^{\prime}(I) \quad$ The derivative of $\lambda_{i}(I)$ with respect to ionic strength.

$\lambda_{M X}^{(0)}, \lambda_{M X}^{(1)}, \lambda_{M X}^{(2)}$

Second order interaction coefficient parameters for cation $M$ and anion $X$.

$\lambda_{w} \quad$ Rational (mole fraction) activity coefficient of water, $a_{w}=\lambda_{w} x_{w}$.

$\lambda_{\text {ow }}$ Ratiunal (mole fraction) activity coefficient : : the o-th end member of the $\psi$-th solid solution.

$E_{\lambda_{M A I^{\prime}}}(I) \quad$ The clectrostatic part of $\lambda_{M M}(I)$.

$s_{\lambda_{M M r}} \quad$ The short-range part of $\lambda_{M M}(I)$; trealed as a constant.

$\mu_{i j} \quad$ Third-order interaction cocficient for the $i$-th, $j$-th, and $k$-th aquecus solute species.

$v_{M} \quad$ Number of cations $M$ produced by dissociatim of the achueous neutral electrolyte $M X$.

$v_{M X} \quad$ Number of cations $M$ and anions $X$ produced by dissociation of the aqueous neutral electrolyte $M X$.

$v_{X} \quad$ Number of anjons $X_{j}$ produced by dissociation of the aqueous neutral clectrolyte $M X$.

$\rho_{g / m l} \quad$ Solution deasity, $g / m l$.

$\sigma, \sigma^{\prime} \quad$ Symools denoting end-member components of a solid solution.

$\sigma_{T, \Psi} \quad$ Total number of end members in the $\psi$-th solid solution.

$7_{s} \quad$ Alkalinity factor, the number of hydrogen ion neutralizing equivalents per mole of the s-th aqueous species.

$\phi \quad$ (a) Subscript denoling a pure mineral; (b) the osmotic cocfficient of the aqueous solution.

$\Phi_{M M^{\prime}}(I) \quad$ Harvie, Mrlier, and Weare's (1984) notation for $\theta_{M M^{\prime}}(I)$.

$\Phi_{M M M}^{\prime}(I) \quad$ Harvie, Moller, and Weare's (1984) notation for $\theta_{M / S J}^{\prime}(I)$.

$\chi_{g} \quad$ Fugacity coefficient of the $g$-th gas.

$\Psi \quad$ Subscript denoting a solid solution.

$\Psi_{T} \quad$ Total number of solid solutions in a system.

WatM'X Observable third order interaction cocfficient for nevtral electuolytes $M X$ and $M^{\prime} X$.

$\Omega \quad$ Water constant; 1000 divided by the nolecular weight of walcr; about 55.51.

$\aleph_{i j} \quad$ Ionic activity combination parankter: $x_{i j}=\left|z_{j}\right| \log a_{i}-\frac{z_{j} z_{j}}{\left|z_{j}\right|} \log a_{j}$

$+\quad$ Subscript denoting a reaction proceeding in the forward sense; the convention in this report equates this with dissociation, dissolution, or destruction of the associated species. 
Subscript denoting a reaction proceeding in the backward sense; the convention in this report equates this with association, precipitation, or formation of the associaled species. 


\title{
EQ3NR, A Computer Program for Geochemical Aqueous Speciation-Solubility Calculations: Theoretical Manual, User's Guide, and Documentation \\ (Version 7.0)
}

\begin{abstract}
EQ3NR is an aqueous solution speciation-solubility modeling code. It is part of the EQ3/6 software package for geochemical modeling. It computes the thermodynamic state of an aqueous solution by determining the distribution of chemical species, including simple ions, ion pairs, and complexes, using standard state thermodynamic data and various equations which describe the thermodynamic activity coefficients of these species. The input to the code describes the aqueous solution in terms of analytical data, including total (analytical) concentrations of dissolved components and such other parameters as the $\mathrm{pH}, \mathrm{pHCl}, E h$, pe, and oxygen fugacity. The input may also include a desired electrical balancing adjustment and various constraints which impose equilibrium with specified pure minerals, solid solution end-member components (of specified mole fractions), and gases (of specified fugacities). The code evaluates the degree of disequilibrium in terms of the saturation index $(S I=\log Q / K)$ and the thermodynamic affinity $(A=-2.303 R T \log$ $Q / K)$ for various reacticns, such as mineral dissolution or oxidation-reduction in the aqueous solution itself. Individual values of $E h, p e$, oxygen fugacity, and $A h$ (redox affinity) are compuled for aqueous redox couples. Equilibrium fugacities are computed for gas species. The code is highly flexible in dealing with various parameters as either model inputs or outputs. The user can specify modification or substitution of equilibrium constants at run time by using options on the input file. The output consists of an output file and a pickup file, which can be used to initialize an EQ6 reaction path caiculation. The chief numerical method employed is a iybrid NewtonRaphson technique. This is supported by a set $c^{f}$ algorithms which creare and optimize starting values. EQ3NR reads a secondary unformatted data file (datal) that is created from a primary formatted data file (dat20) by EQPT, the EQ3/6 data file preprocessor. There is currently a set of five data (data0) files. Three of these may be used with either the Davies equation or the B-dot equation to describe the activity coefficients of the aqueous species. Their use is restricted to modeling dilute solutions. The other two of these use Pitzer's equations and are suitable for modeling soiutions to high concentrations, though with fewer chemical components. The temperature range of the thermodynamic data on the data files varies from $25^{\circ} \mathrm{C}$ only to $0-300^{\circ} \mathrm{C}$. EQ3NR may be used by itself or to initialize a reaction path calculation by EQ6, its companion code in the EQ3/6 package. EQ3NR and the other codes in the EQ3/6 package are written in FORTRAN 77 and have been developed to run under the UNIX operating system on computers ranging from workstations to supercomputers.
\end{abstract}

\section{Introduction}

EQ3NR is a speciation-solubility code for modeling the thermodynamic state of an aqueous solution. In essence, this involves a static calculation that is usually based on water chemistry an analysis. The purpose of such a calculation is usually to find the detailed distribution of chemical species and to assess the degree of equilibrium (or disequilibrium) peraining to various reactions, usually those involving other phases. EQ3NR can not be used to directly model the 
chemical evolution of such a water. However, it can be used to initialize such a calculation, which can be made by the companion code EQ6 (Wolery and Daveler, 1992).

EQ3NR is part of the EQ3/6 software package (see Wolery, 1992). This repon describes EQ3NR in version 7.0 (version 3245.1090 in the old numbering system) of this package (see the EQ3/6 Package Overview and Installation Guide, Wolery, 1992). Other codes in the package include EQPT (Daveler and Wolery, 1992), a data file preprocessor, and EQ6 (Wolery and Daveler, 1992), a reaction path code. The relationship of the EQ3NR cose to EQ6, EQPT, and the set of supporting thermodynamic data files is shown in Figure 1 . This figure depicts the flow of information involving these codes. At present, there are five distinct data files, denoted by the suffixes com, sup, nea, hmw, and pit. These are provided in formatted ASCII and are called data0 files. EQPT processes these one at a time (looking for a file named simply data0, though these files are normally stored under names which include the relevant suffixes) and writes a corresponding unformatted data file, which is called simply datal. These are also normally stured under names including the relevant suffixes. To run EQ3NR or EQG, the user must provide one of these files, which is known to each code simply as datal.

The user must select which of the five data files is most appropriate to a given problem. Each data file corresponds to a general formalism for treating the activity coefficients of the aqueous species and contains the relevant activity coeficient data as well as standard state thermodynamic data. The activity coefficient formalisms currently built into EQ3/6 are discussed in Chapter 3 . The com, sup, and nea data files are specific to a general extended Debye-Hückel formalism and can be used by EQ3NR and EQ5 with either the Davies (1962) equation or the B-dot equation (Helgeson, 1969). These equations are only valid in relatively dilute solutions. The hmw and pit data files are specific to the formalism proposed by Pitzer $(1973,1975)$ and can be used to model solutions extending to high concentrations. However, the scope of chemical components covered is smaller. The temperature limits on the data files also vary, from $25^{\circ} \mathrm{C}$ only to $0-300^{\circ} \mathrm{C}$.

Some important data file characteristics are given in Table 1. The com (for "composite") data file is the largest of the three data files specific to the extended Debye-Hückel formalism. It is a product of Lawrence Livermore National Laboratory (LLNL) drawing on many data sources, including those on which the other four data files are based. The sup data file is based entirely on SUPCRT92 (Johnson, Oelkers, and Helgeson, 1992), a data base and program for dealing with thermodynamic data based on the work of Helgeson and Kirkham (1974ab, 1976), Helgeson et al. (1978), Tanger and Helgeson (1988), Shock and Helgeson (1988, 1989, 1990), Shock, Helgeson, and Sverjensky (1989), Johnson and Noron (1991), and Shock et al. (1992). The nea data file is based entirely on Grenthe et al. (1989, draft report), a product of the Data Bank of the Nuclear Energy Agency of the Europenn Community. This report has recently been published as Grenthe et al. (1992)The hmw data file is based on Harvie, Mqller, and Weare (1984). The pit data file is based mostly on data summarized by Pitzer (1979). All five data files are maintained at LLNL in a relational data base described by Delany and Lundeen (1991). This relational data base is part of the Yucca Mountain Site Characterization Project s Technical Data Base.

The sup data file has a high level of internal consistency among the standard state thermodynamic data. In addition, the temperature-pressure dependence of these data are represented by a suite of equations of state for minerals, gases, and aqueous species that are well established in the geochemical literature (see references noted above). This data file covers a wide range of 


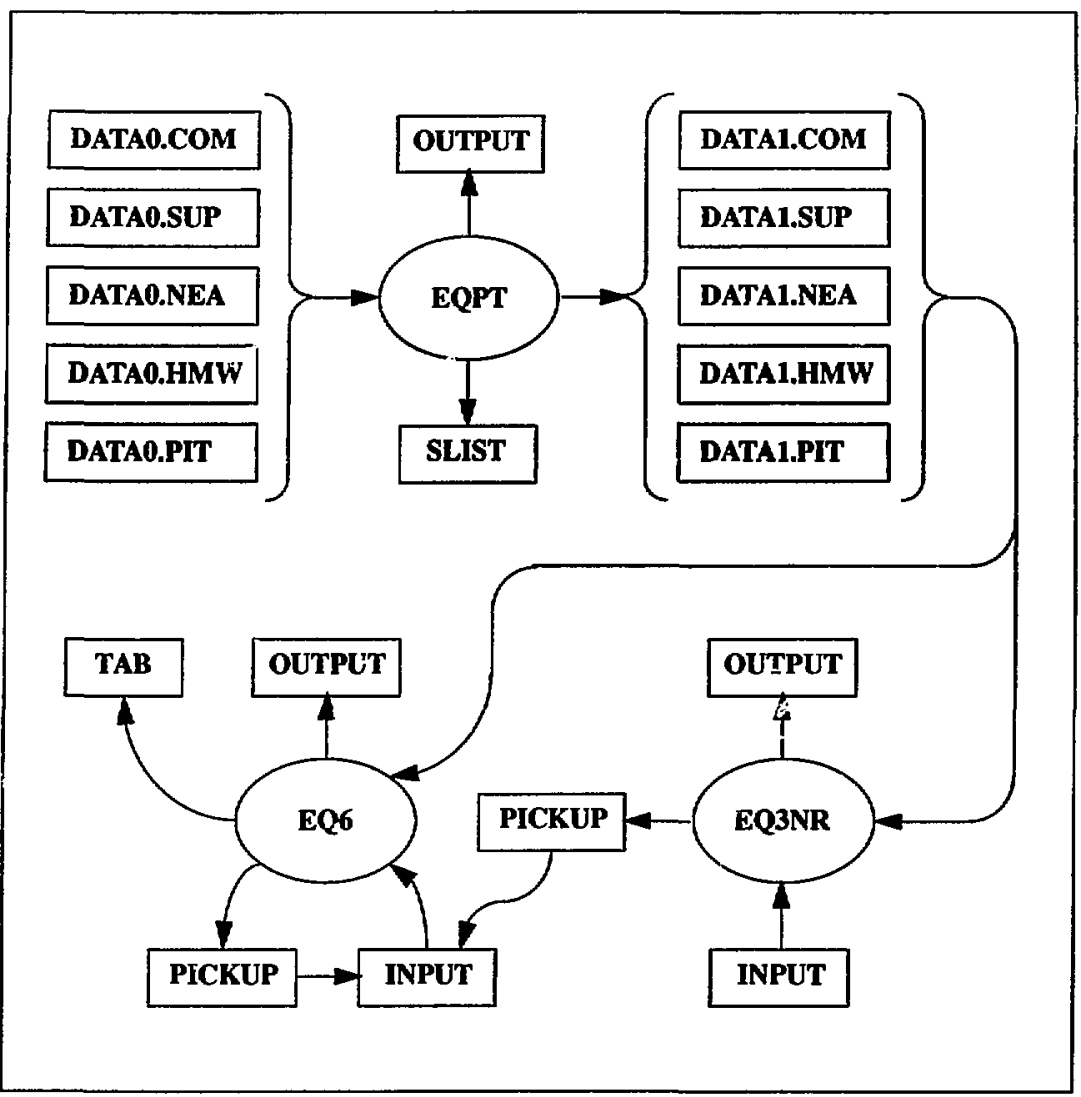

Figure 1. The flow of information among the computer codes EQPT, EQ3NR, and EQ6. Computer codes are represented by ovals, files by rectangles.

chemical elements and species of interest in the study of rock/water interactions (e.g., components which make up the major rock-forming and ore-forming minerals). It also includes a large number of organic species, mostly of small carbon number $\left(C_{2}-C_{8}\right)$. The nea data file is something of a specialty item. Its strongest point is a thorough representation of the thermodynamics of uranium species.

The com (composite) data file encompasses a much broader range of chemical elements and species. It includes the data found on the sup and nea data files, with preference given to data from 
Table 1. Major characteristies of the cument (ive EO3/6 dala files ("R10" versions).

\begin{tabular}{|c|c|c|c|c|c|c|c|c|c|}
\hline $\begin{array}{l}\text { File Nanw } \\
\text { (Suftix) }\end{array}$ & Sultec & $\begin{array}{l}\text { Aclivity } \\
\text { Coefficicnt } \\
\text { Fomalitm }\end{array}$ & $\begin{array}{l}\text { Teniperature } \\
\text { Linvits }\end{array}$ & $\begin{array}{l}\text { Number of } \\
\text { Cliemical } \\
\text { Elemet.'s }\end{array}$ & $\begin{array}{l}\text { Number of } \\
\text { Basis } \\
\text { Species }\end{array}$ & $\begin{array}{l}\text { Number of } \\
\text { Aquteous } \\
\text { Species }\end{array}$ & $\begin{array}{l}\text { Number of } \\
\text { Pure } \\
\text { Minerals }\end{array}$ & $\begin{array}{l}\text { Number of } \\
\text { Solid } \\
\text { Solutions }\end{array}$ & $\begin{array}{l}\text { Number of } \\
\text { Gas } \\
\text { Specics }\end{array}$ \\
\hline $\operatorname{con}$ & $\begin{array}{l}\text { GEMBOCHS } \\
\text { (LLNL) }\end{array}$ & $\begin{array}{l}\text { Extended } \\
\text { Debye. } \\
\text { Hackel }\end{array}$ & $0-300^{\circ} \mathrm{C}$ & 78 & 147 & $8 \$ 2$ & 886 & 12 & 76 \\
\hline sup & SUPCRTY2 & $\begin{array}{l}\text { Extended } \\
\text { Debye. } \\
\text { Holekel }\end{array}$ & $0-300^{\circ} \mathrm{C}$ & 69 & 105 & 315 & 130 & 0 & 16 \\
\hline med & $\begin{array}{l}\text { NEA draft } \\
\text { repon }\end{array}$ & $\begin{array}{l}\text { Extended } \\
\text { Debye. } \\
\text { Hirckel }\end{array}$ & $0-300^{\circ} \mathrm{C}$ & 32 & so & 158 & 188 & 0 & 76 \\
\hline loniw & $\begin{array}{l}\text { Harvie, Moller, } \\
\text { and Weare } \\
(1984)\end{array}$ & $\begin{array}{l}\text { Piver's } \\
\text { Equations }\end{array}$ & $25^{\circ} \mathrm{C}$ ouly & 9 & 13 & 17 & 51 & 0 & 3 \\
\hline pit & Pitzer (1979) & $\begin{array}{l}\text { Piker's } \\
\text { Equations }\end{array}$ & $0-100^{\circ} \mathrm{C}$ & 52 & 62 & 68 & 381 & 0 & 38 \\
\hline
\end{tabular}


the former in cases of overlap. It also includes some data found in the hmw data file, as well as other data which do not appear in any of the other data files. Some of these data are estimates based on correlations or extrapolations (as to higher temperature), and are not tied directly to experimental measurements. The com data file thus represents a melarige of data, which by its nature offers less assurance of internal consistency. However, this offers the only means presently available for modeling aqueous solutions with a high degree of compositional complexity, such as the fluids expected to be found in and about a facility for the geologic disposal of industrial or nuclear waste (e.g.: the potential repository for high-level nuclear waste at Yucca Mountain, Nevada).

The hmw data file has the highest degree of internal consistency of any of the five data files, including mutual consistency of activity coefficient data and standard state thermodynamic data. It can be applied to dilute waters or concentrated brines. However, it only treats the se! of components present in the "sea-salt" system (the major cations and anions present in seawater, including carbonate and bicarbonate). The geochemically important components aluminum and silica are not included. Also, this data file is limited to a semperature of $25^{\circ} \mathrm{C}$. The pit data file can also be applied to concentrated brines. It covers a larger set of components, tut these mostly involve other cations and anions of strong electrolytes. Examples include lithium and bromide. This data file nominally covers the temperature range of $0-100^{\circ} \mathrm{C}$. However, it represents a melange of data, not a carefully crafted intemally consistent set.

The data file preprocessor EQPT (Daveler and Wolery, 1992) performs a number of functions. It checks the composition, charge, and reaction coefficient data on a data 0 file for internal consistency and fits interpolating polynomials to various temperature dependent data which are organized on the data0 file on temperature grids. Such data include certain aqueous species activity coefficient parameters, such as Debye-Hückel $A_{\gamma, 10}$ and $B_{\gamma}$ and the equilibrium constants for the reactions represented on the data file. In addition, in the case of data files specific to the formalism of Pitzer's equations, observable interaction coefficients are mapped to a set of conventionally defined primitive interaction coefficients (see Chapter 3). EQPT then writes the datal file corresponding to the input data0 file. For details of the contents and structure of data 0 and datal files, see Daveler and Wolery (1992). Run-time alteration of the values of selected equilibrium constants can subsequently be selected by the user on the EQ3NR input file (see Chapter 6). EQPT also writes to a screen file and an output file, both of which are generally significant only if an error condition is encountered. In addition, it writes an slist (species list) file. This is very useful to the user, as it lists the species that are represented on the data file and identifies which species are in the strict and auxiliary basis sets (See Chapter 5).

A speciation-solubility problem to be run with EQ3NR is described on the EQ3NR input file. This is the subject of Chapter 6 of this report. Examples are presented in Chapter 7. The code then produces an output file describing the results of the calculation. While the code is nunning, it writes to a screen file, primarily to appijse the user of what is happening. It also writes a pickup file, which contains a compact description of the aqueous solution (see Chapter 8 ). This is required for a subsequent EQ6 calculation; it corresponds to the bottom part of the EQ6 input file. It has no other real use. EQ6 in turn writes its own output file, as well as a tab file which contains certain data in tabular form suitable for supporing local graphics postprocessing. This code also writes to the screen file while it is running. In addition, EQ6 writes its own pickup file, which 
may be used as an input file to restart a reaction path calculation from the point at which a previous run stopped.

The input to the code consists of a chemical analysis of a water and specification of various userdefined options. The input usually consists mostly of analytical yalues for concontrations of dissolved components. These represent total values that do not distinguish between contributions from simple ions, ion pairs, and aqueous complexes, species which may exist in solution in mutual equilibrium. In addition, analytical data may or may not distinguish a dissolved component by oxidation state. The $p H$ is also normally an input parameter. A new altemative parameter called $\mathrm{pHCl}$ can be input in place of $\mathrm{pH}$ to overcome the liquid junction potential problem in measuring $p H$ in concentrated solutions (see Chapter 2 ). The $E h$ (redox potential) is also a common input parameter, though its usage is somewhat problematical (see Chapter 2). One may specify the oxygen fugacity or pe instead, though this is no less problematical. It is also possible to specify a redox couple to define the redox state. For example, one might specify the ferrous-ferric couple if one had two total concentration values, one for $\mathrm{Fe}^{2+}$ and another for $\mathrm{Fe}^{3+}$. It is best to treat as many couples as possible by this method. That way, redox equilibrium can be tested instead of merely assumed.

The basic input constraints (total concentrations, $p H$, etc.) are associated on a one-to-one basis with master or basis species. Basis species (see Chapters 2 and 5) represent the chemical components of the aqueous solution. They also function as basic elements for writing chemical reactions in a standardized format that is convenient for chemical modeling. The solvent, water, is a basis species, but is an exception in a speciation-solubility problem in that no input constraint is associated with it. The basis species used to write oxidation-reduction reactions in EQ3/6 is oxygen gas, which is treated as a fictive aqueous species. An input for it is required only if the problem has a redox aspect. The other basis species consist of simple species such as $\mathrm{Na}^{+}$and $\mathrm{Cl}$ and a few more complex species such as $\mathrm{SO}_{4}{ }^{2}$. A minimum basis set has one species representing each chemical element and its associated mass balance, plus one more representing oxidation-reduction and charge balance. The minimum basis is called the strict basis. EQ3NR also has an auxiliary basis, which consists of species which are related vin associated chemical reactions to the strict basis species, but for which the user may choose to impose constraints other than equilibrium with the latter. Most auxiliary basis species represent a chemical element in a different $\mathrm{ox}_{\mathrm{x}}$ idation state.

If desired, the concentration of a specified ion may be adjusted to satisfy electrical balance. $A_{n}$ option to constrain the carbonate system by specifying the alkalinity has been deleted from the present version of EQ3NR. The reasons behind this action and suggestions for altemative medsures are discussed in Chapter 2. It is also possible to constrain various species by certain equilibrium assumptions instead of analytical data. For example, the concentration of dissolved calcium may be constrained to satisfy equilibrium with calcite. It is also possible to constrain the concentration of a species to satisfy equilibrium with a solid solution end-member component of specified mole fraction. Similarly, the concentration of a species may be constrained to satisfy equilibrium with a gas species of specified fugacity.

EQ3NR computes the distribution of chemical species present in the model. Essentially, this involves partitioning the input total concentrations. The code thus determines the concentrations, 
activity coefficients, and thermodynamic activities of all species present. This in tum permits evaluation of the saturation indices $(S I=\log Q / K$, where $Q$ is the activity product and $K$ the equilibrium constant) and thermodynamic affinities $(A=-2.303 R T \log Q / K$, where $R$ is the gas constant and $T$ the absolute temperature) of various reactions, chiefly for the dissolution of minerals. However, these functions are also evaluated for certain reactions occurring internally in the aqueous solution and which are normally only assumed to be in thuilibrium (the input file requires additional data to do this). In the case of aqueous redox reactions, the theoretical $E h, p e$, oxygen fugacity, and redox affinity $(A h)$ are computed. Differences in the values of these corresponding parameters for two redox couples are measures of the degree of disequilibrium between them. The equilibrium fugacities of various gas species are also determined.

The results of these calculations depend on the supporting data read from the data file. The use of different data files may give different results. Different results may be obtained not only because of the use of different values of standard state thermodynamic data (e.g., equilibrium constants), but also by different choices in the set of equations for the activity coefficients as well as the use of different values in the choice of parameters appearing in these equations (e.g.. the Debye-Hückel $A_{\phi}$ parameter, various kinds of interaction coefficients). The equations for calculating th: : activity coefficients of aqueous species are discussed in Chapter 3 . The equations for calculating the activity coefficients of end-member components of solid solution phases are discussed in Chapter 4. In speciation-solubility calculations, these latter equations and their supporting data normally affect only the saturation indices calculated for solid solutions. However, they do affect the computed aqueous speciation model if one of the defining model constraints assumes equilibrium with a solid solution end-member component.

In some modes, such as when the concentration of a species is adjusted to satisfy electrical balance or to satisfy an equilibrium constraint, the code actually computes part of what would normally be analytical data. In this mode, for example, the code can be used to calculate recipes for custom $p H$ buffers. An example of this is included in Chapter 7. Calculations using such constraints can be somewhat dangerous, especially when used in combination. It is not hard to construci problems that have no physical solutions. In such cases, the code can of course compute no corresponding answers, but it does a generally good job of diagnosing the problems and informing the user of the nature of the problem.

In general, the code is highly flexible in that the roles of many parameters as inputs and outputs can be reversed. There are very few restrictions on the input combinations that may be defined by the code user. The main requirement is that the problem must have a realistic answer.

EQ3NR uses a highly efficient hybrid Newton-Raphson algorithm in which the activity coefficients of the aqueous species are held constant in a Newton-Raphson step and re-adjusted between such steps. The code features both user-controlled and automatic basis-switching, a procedure for rewriting reactions and redefining the set of basis species. These features are occasionally necessary to induce the iterative calculations to converge. The code creates its own starting estimates for Newton-Raphson iteration, and uses a first order algorithm in addition to possible automatic basis switching to optimize these before beginning Newton-Raphson iteration. The numerical methods used by the code are discussed in Chapter 9 . 
EQ3NR performs a number of tests on the model constraints to see if they make sense. It first checks the data and options read from the input file for inconsistent or incomplete combinations. It will write informative error messages and terminate any further action if it detects bad input. However, not all bad input can be detected at this stage. Further analysis takes place when the code chooses starting estimates for the master iteration variables. Finally, if Newton-Raphson iteration fails to converge, EQ3NR will analyze the results to generate crash diagnostics. Most of these will point to bad input, usually input that is bad in more subtle ways than those which would have been flagged earlier.

The code architecture is described in Chapter 10. Appendix A contaits a glossary of the major code variables. The source code mociules are listed and briefly described in Appendix B (for a similar treatment of EQLIB modules, see Appendix B of the EQ3/6 Package Overview and lnstallation Guide, Wolery, 1992). Appendix C contains a list of error messages generated by EQ3NR modules, along with related notes (see Appendix C of Wolery, 1992, for a similar list for EQLIB rodules). Appendix D contains notes pertaining to known bugs and such.

EQ3NR and the other codes in the EQ3/6 software package are written in FORTRAN 77 and have been developed to run under UNIX operating systems on computers ranging from workstations to supercomputers, including Sun SPARCstations, VAXes (ULTRIX operating system), Alliants (CONCENTRIX operating system), and Crays (UNICOS operating system). They are fairly readily portable to VAX computers running the non-UNIX VMS operating system. They may be portable as well to 386 and 486 PCs. Platforms used at LLNL include Sun SPARCstations and an Alliant FX/80. For details concerning platforms, see the EQ3/6 Package Overview and Installation Guide (Wolery, 1992). 
activity coefficients, and thermodynamic activities of all species present. This in tum permits evaluation of the saturation indices $(S I=\log Q / K$, where $Q$ is the activity product and $K$ the equilibrium constant) and thermodynamic affinities $(A=-2.303 R T \log Q / K$, where $R$ is the gas constant and $T$ the absolute temperature) of various reactions, chiefly for the dissolution of ininerals. However, these functions are also evaluated for certain reactions occurring internally in the aqueous solution and which are normally only assumed to be in equilibrium (the input file requires additional data to do this). In the case of aqueous redox reactions, the theoretical $E h$, pe, oxygen fugacity, and redox affinity $(A h)$ are computed. Differences in the values of these corresponding parameters for two redox couples are measures of the degree of disequilibrium between them. The equilibrium fugacities of various gas species are also determined.

The results of these calculations depend on the supporting data read from the data file. The use of different data files may give different results. Different results may be obtained not only because of the use of different values of standard state thermodynamic data (e.g., equilibrium constants), but also by different choices in the set of equations for the activity coefficients as well as the use of different values in the choice of parameters appearing in these equations (e.g., the Debye-Hückel $A_{\phi}$ parameter, various kinds of interaction coefficients). The equations for calculating the activity coefficients of aqueous species are discussed in Chapter 3 . The equations for calculating the activity coefficients of end-member components of solid solution phases are discussed in Chapter 4. In speciation-solubility calculations, these latter equations and their supporting data normally affect only the saturation indices calculated for solid solutions. However, they do affect the computed aqueous speciation model if one of the defining model constraints assumes equilibrium vith a solid solution end-member component.

In some modes, such as when the concentration of a species is adjusted to satisfy electrical balance or to satisfy an equilibrium consuraint, the code actually computes part of what would normally be analytical data. In this mode, for example, the code can be used to calculate recipes for custom $p H$ buffers. An example of this is included in Chapter 7. Calculations using such constraints can be somewhat dangerous, especially when used in combination. It is not hard to construct problems that have no physical soidtions. In such cases, the code can of course compute no corresponding answers, but it does a generally good job of diagnosing the problems and informing the user of the nature of the problem.

In general, the code is highly flexible in that the roles of many parameters as inputs and outputs can be reversed. There are very few restrictions on the input combinations that may be defined by the code user. The main requirement is that the problem must have a realistic answer.

EQ3NR uses a highly efficient hybrid Newton-Raphson algorithm in which the activity coeffcients of the aqueous species are held constant in a Newton-Raphson step and re-adjusted between such steps. The code features both user-controlled and automatic basis-switching, a procedure for rewriting reactions and redefining the set of basis species. These features are occasionally necessary to induce the iterative calculations to converge. The code creates its own starting estimates for Newton-Raphson iteration, and uses a first order algorithm in addition to possible automatic basis switching to optimize these before beginning Newton-Raphson iteiation. The numerical methods used by the cole are discussed in Chapter 9. 
EQ3NR performs a number of tests on the model constraints to see if they make sense. It first checks the data and options read from the input file for inconsistent or incomplete combinations. It will write informative error messages and terminate any further action if it detects bad input. However, not all bad input can be detected at this stage. Further analysis takes place when the code chooses starting estimates for the master iteration variables. Finally, if Newton-Raphson iteration fails to converge, EQ3NR will analyze the results to generate crash diagnostics. Most of these will point to bad input, usually input that is bad in more subtle ways than those which would have been flagged earlier.

The code architecture is described in Chapter 10. Appendix A contains a glossary of the major code variables. The source code modules are listed and briefly described in Appendix B (for a similar treatment of EQLIB modules, see Appendix B of the EQ3/6 Package Overview and Installation Guide, Wolery, 1992). Appendix C contains a list of error messages generated by EQ3NR modules, along with related notes (see Appendix C of Wolery, 1992, for a similar list for EQLIB modules). Appendix D contains notes pertaining to known bugs and such.

EQ3NR and the other codes in the EQ3/6 software package are written in FORTRAN 77 and have been developed to run under UNIX operating systems on computers ranging from workstations to supercomputers, including Sun SPARCstations, VAXes (ULTRIX operating system), Alliants (CONCENTRIX operating system), and Crays (UNICOS operating system). They are fairly readily portable to VAX computers running the non-UNIX VMS operating system. They may be portable as well to 386 and 486 PCs. Platforms used at LLNL include Sun SPARCstations and an Alliant FX/80. For details concerning platforms, see the EQ3/6 Package Overview and Installation Guide (Wolery, 1992). 


\section{Speciation-Solubility Modeling of Aqueous Systems}

\subsection{Introduction}

EQ3NR is a speciation-solubility code for aqueous systems. As such, given sufficient data on a specific aqueous system, it computes a model of the solution which consists of two principal parts: the distribution of species in the solution and a set of saturation indices $(S I=\log Q / K)$ for various reactions of interest. The saturation indices are measures of the degree of disequilibrium of the corresponding reactions. They provide a means of searching for solubility controls on natural waters. For example, if a series of related fluids all have calcite $S I$ values close to zero, it is probable that this mineral is present and partial equilibrium with it is maintained as the solutions evolve in composition.

EQ3NR is not a computerized gcochemical model, but a code which is capable of evaluating geochemical models which are defined by the contents of a supporting data file (of which there are now five to choose from) and by other assumptions which the user sets on the EQ3NR input file. The supporing data files differ not only in terms of data values, but more importantly in terms of the identities of the coinponents and chemical species represented and in terms of the general approaches to dealing with the problem of activity coefficients. Because of various limitations, some problems may require the use of only certain data files, while others can be treated using any of the available data files. The user must choose the best data file (or files) with which to run a particular problem. The user must also understand both the particular problem and the code capabilities and limitations well enough to construrt an adequate input file.

Although speciation-solubility models are commonly used as a means of testing whether or not heterogeneous reactions are in a state of thermodynamic equilibrium, they often just assume that all reactions occurring in aqueous solution are in such a state. Such reactions most likely to be in disequilibrium are redox reactions or reactions for the formation or dissociation of lange complexes that are more like small polymers, such as $\left(\mathrm{UO}_{2}\right)_{3}(\mathrm{OH})_{7}$. Speciation-solubility models are better used when they are employed to test the degree of disequilibrium of these kinds of reactions than when they are forced to assume that such reactions are in equilibrium.

A speciation-solubility model can not by itself predict how aqueous solution composition will change in response to rock/water interactions. Nevertheless, this type of modeling can be a powerful tool for elucidating such interactions when it is applied to a family of reiated waters. Such a family might be a set of spring waters issuing from the same geologic formation, a sequence of ground water samples taken from along an underground flow path, or a sequence of water samples taken in the course of a rock/water interactions experiment in the laboratory. Jenne (1981) reviews several studies of this kind. Particularly interesting are Nordstrom and Jenne's (1977) study of fluorite solubility equilibria in geothermal waters and Nordstrom, Jenne, and Ball's (1979) study of controls on the concentration of iron in acid mine waters.

EQ3NR offers many options for the input file description of the composition of a given water. Consequently, the code can be used in at variety of ways. Many of the descriptive parameters of interest can be eitner model inputs or outputs. For example, the $\mathrm{pH}$ of a buffer solution can be calculated from the buffer recipe by adjusting the hydrogen ion concentration to satisfy charge balance. Altematively, adjusting the concentration of a buffer component to satisfy the charge 
balance is a means of computing the complete recipe for a buffer having a desired $\rho H$. Some of the possible model inputs are assumptions, as of equilibrium with specifiei minerals. The use of some types of model inputs also pose special problems, some of which occur in particular contexts. The worst of these pertain to $E h$, alkalinity, and $p H$ and will be discussed in some detail later in this chapter.

\subsection{Units of Concentration}

EQ3NR uses the molal scale as the principal unit of concentration for aqueous species. The molal concentration (molality) of a substance dissolved in water is defined as:

$$
m_{i}=\frac{n_{i}}{w_{w}}
$$

where $n_{j}$ is the number of moles of the $i-$ th solute species and $w_{w}$ is the number of kilograms of solvent water. Other common measures of aqueous solute concentration are the molarity (inoles of substance per liter of aqueous solution), the part-per-million or ppm by volume ( $\mathrm{mg} / \mathrm{L}$, milligrams of substance per (iter of solution), and the ppm by weight (mg/kg, milligrams of substance per kilogram of solution). The EQ3NR code accepts concentration parameters in any of these units (see Chapter 4), but converts non-molal concentrations to molalities before computing the aqueous speciation model. Whether or not it does this correctly depends on circumstance and data provided by the user.

The conversion equations in all three cases require a value for the total dissolved salts in $\mathrm{mg} / \mathrm{kg}$ solution $\left(C_{T S, m g / k g}\right)$. The density of the aqueous solution in $\mathrm{g} / \mathrm{ml}\left(\rho_{g / n l}\right)$ is also required to convert molarities and $\mathrm{mg} / \mathrm{L}$ concentrations to molalities. The total dissolved salts in $\mathrm{mg} / \mathrm{kg}$ may be calculated from the total dissolved salts in $\mathrm{mg} / \mathrm{L}\left(C_{T S, \mathrm{mg} / \mathrm{L}}\right)$ and the density according to:

$$
C_{T S, m g / k g}=\frac{C_{7 \mathrm{~S}, m g / L}}{\rho_{g / m l}}
$$

EQ3NR expects values of $C_{T S, ~ m g / k g}$ and $\rho_{g / m l}$ on the input file if such conversions are necessary (see Chapter 4). In place of $C_{T s, m g l k g}$, one may enter $C_{T S, m g / L}$ and $\rho_{g i m l}$ and $C_{T s, ~ m g / k g}$ is calculated from the above equation. If such values are not provided, $C_{T S}, \mathrm{mg} / \mathrm{kg}$ is assigned a default value of zero and $\rho_{g / m l}$ is assigned a default value of unity. These values are generally adequate for dilute solutions at temperatures near $25^{\circ} \mathrm{C}$. In the case of brines, these values are not adequate, and the user must provide actual values as pan of the input in order to obtain accurate conversion. The code provides no checks or warnings if these are not provided.

The weight fraction of solvent water is given by:

$$
N_{\mathrm{w}}=\frac{1,000,000-C_{\text {Ts.mg/kg }}}{1,000,000}
$$

Letting $C_{i, \text { molar }}$ be the molar concentration of the $i$-th solute species, the molality is gi: en by 


$$
m_{i}=\frac{C_{i, m o ! a r}}{\rho_{g i m i} N_{w}}
$$

Letting $C_{i, m g / L}$ be the concentration in $\mathrm{mg} / \mathrm{L}$, the conversion is:

$$
m_{i}=\frac{0.001 C_{i, m g / L}}{p_{g / m l} M_{w} N_{w}}
$$

where $M_{w}$ is the molecular weight of the solvent, vater $\left(M_{w} \approx 18.015 \mathrm{~g} /\right.$ mole $)$. Letting $C_{i, \mathrm{mg} / \mathrm{kg}}$ be the concentration in $\mathrm{mg} / \mathrm{kg}$ solution, the conversion is:

$$
m_{i}=\frac{0.001 C_{i, m g / L}}{M_{w} N_{w}}
$$

Some dissolyed gas analyses are reported i:l units of $\mathrm{ml}$ (STP)/ml solution, where STP refers to standard temperature and pressure $\left(0^{\circ} \mathrm{C}\right.$ and $\left.1 \mathrm{~atm}\right)$. The conversion equation is:

$$
m_{i}=\frac{1000 C_{i, m l(S T P X m l}}{V}
$$

where $V_{g}$ is the molar volume of an ideal gas at $\operatorname{STP}\left(V_{\mathrm{g}}=22,413.6 \mathrm{ml} / \mathrm{mole}\right)$.

The concentration of solvent water is defined as its mole fraction:

$$
x_{w}=\frac{n_{w}}{n_{w}+\sum_{i} n_{i}}
$$

where $n_{w}$ is the number of moles of water. The molality of the $i$-th solute species can also be written as:

$$
m_{i}=\frac{\Omega n_{i}}{n_{w}}
$$

where $\Omega$ is the number of moles of water comprising a mass of $1 \mathrm{~kg}\left(\Omega=55.51 ; \Omega w_{w}=n_{w}\right)$. Substituting this relation into the one above it gives:

$$
x_{w}=\frac{\Omega}{\Omega+\sum_{i} m_{i}}
$$

EQ3NR uses this relation to calculate the mole fraction of water: This is done in a self-consistent manner in the iteration process. Thus, the user is not required to input a value. 
A similar self-consistent treatment could be implemented to handle both $C_{T S}$, mg/kg and she solution densiry. However, no such treatment exists in the current version of EQ3NR, nor any other such modeling code known to the present writers. Implementation of a self-consistent treatment of the solution density would require the addition of models for partial molar volumes to the code and incorporation of the correspinding equations in the iteration process. The theoretical and practical aspects of partial molar volumes in solutions extending to high concentration have been addressed for example by Millero (1977), Helgeson, Kirkham, and Flowers (1981), Kumar (1986), Connaughton, Millero, and Pizzer (1989), and Monnin (1989).

\subsection{Input Constraints, Governing Equations, and Outputs}

\subsubsection{Overview}

Aqueous speciation models can be constructed to satisfy a wide variety of combinations of possible input constraints and governing equations. The input constraints may include total (analytical) concentrations, an electrical balance requirement, free concentrations, activities, $p H, E h, p e$, oxygen fugacity, phase equilibrium requirements, homogeneous equilibria, and run-specific values for equilibrium constants. The goveming equations are the corresponding mathematical expressions. such as the mass balance equation and the charge balance equation.

The choice of governing equations in large part depends on waich parameters are to be inputs to the model and which are to be outputs. This, in tum, is a function of what data on a given water are available, what form they are in, and what assumptions the modeler would like to use.

Chemical analysis provides mainly a set of values for the so-called total concentrations of dissolved components. The analytical value for an ion such as calcium is an example. It does not discriminate between the various calcium species in solution, but rather estimates the dissolved calcium contributed by all of them. This leads to a mass balance equation of the form:

$$
m_{T_{,} \mathrm{Ca}^{2+}}=m_{\mathrm{Ca}^{2+}}+m_{\mathrm{CaOH}_{(a q)}}+m_{\mathrm{CaCO}_{3(a q)}}+m_{\mathrm{CaHCO}_{3}^{+}}+\ldots
$$

where $m_{\mathrm{Ca}^{2+}}$ is the total or analytical concentration (on the molal scile) and $m_{i}$ is the molality of any individual chemical species contributing to the mass balance. The summations must be weighted by the appropriate stoichiometric equjvalences; e.g., in the case of $F$, one has:

$$
m_{T, F}=m_{F}+m_{H F(a q)}+2 m_{H_{2} f_{2(a q)}}+2 m_{H F_{2}}+3 m_{A \mid F_{3(a q)}}+\ldots
$$

The total concentration is the most common type of input parameler to an aqueous speciation model. The mass balance constraint, which corresponds to it, is therefore the most common goveming equation. As we shall see, there are situations in which a total concentration is replaced by another type of input. In these cuses, the mass balance constraint is replaced by a different governing equation, and the total concentration becomes something to be calculated (an output parameter).

From a purely mathematical point of view, there is no reason to discriminate among ion pairs (and ion-triplets, etc.) and complexes. For some investigators, the term "ion pair" implies at spe- 
cies in which an anion is separated from a cation by an unbroken hydration sheath about the latter, whereas the term "complex" implies direct contact and perhaps some degree of covalent bonding. Other investigators use these ie ms interchangeably. It is a general assumption in cases of geochemical interest that the concentrations of ion-pairs and complexes are governed by thermodynamic equilibrium.

Each case of this equilibrium can be represented by a mass-action equation for the dissociation of the ion-pair or complex. An example will illustrate this. The calcium sulfate ion-pair dissociates according to the reaction:

$$
\mathrm{CaSO}_{4(a q)}=\mathrm{Ca}^{2+}+\mathrm{SO}_{4}^{2-}
$$

where "=" is used as the sign for a reversible chemical reaction. The corresponding mass action equation is:

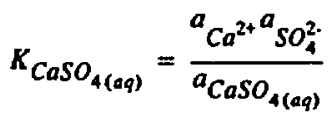

where $K$ is the equilibrium constant and $a_{i}$ represents the thermodynamic activity of each species. This may also be written in logarithmic form:

$$
\log _{\mathrm{CaSO}_{4(\mathrm{aq})}}=\log _{\mathrm{Ca}^{2+}}+\log \mathrm{SOO}_{4}^{2-}-\log a_{\mathrm{CaSO}_{4(a q)}}
$$

The thermodynamic activity is related to the molal concentration by the relation:

$$
a_{i}=m_{i} \gamma_{i}
$$

where $\gamma_{i}$ is the activity coefficient, a function of the composition of the aqueous solution. As the solution approaches infinite dilution, the value of $\gamma_{i}$ for each species approaches unity. The set of equations for computing the activity coefficients of aqueous species is chosen by the user on the EQ3/6 input file (by means of the iopgl option switch). The requisite supporting data are on the EQ3/6 data file. The various formulations presently treated by EQ3/6 are discussed in Chapter 3 .

The following subsections discuss the formulation of aqueous speciation problems in general terms. The rigorous mathematical development is presented in Chapter 9. How to implement these models in EQ3NR is the subject of Chapter 6, and examples are presented in Chapter 7.

\subsubsection{Reference Formulation of the Aqueous Speciation Problem}

In general terms, setting up an aqueous speciation model involves choosing $\boldsymbol{n}$ unknowns and $\boldsymbol{n}$ governing equations. The EQ3NR code offers a very wide range of options in this regard. In order to make sense of the different ways of setting up a model, we define a reference formulation for the aqueous speciation problem. This reference formulation serves as a springboard for discussing what goes into speciation models, what comes outh and what the options are. It is also used to compare hcw the aqueous speciation problem is formulated in EQ3NR (and other speciationsolubility codes in general) with how it is formulated in a reaction-path code like EQ6. 
In the reference formulation, we assume that the activity coefficients are known parameters (the numerical treatment of these is discussed in Chapter 9). Note that the molal concentration of the solvent is fixed as the number of moles of water in a one kilogram mass of the pure substance.

We assume that there are $\varepsilon_{T}$ chemical elements in the model. In order to further simplify the reference formulation, we assume that each element is present in only one oxidation state. Suppose that chemical analysis has given us $\varepsilon_{T}-2$ total concentration values, each for a chemical species, each of which corresponds to a chemical element other than oxygen and hydrogen (e.g.., $\mathrm{Na}^{+}$for $\mathrm{Na}, \mathrm{SO}_{4}{ }^{2-}$ for $S$ ). That gives $\varepsilon_{T}-2$ mass balance equations as goveming equations.

The charge balance equation plays the role that might have been played by a mass balance equation for hydrogen. The charge balance equation may be written in the general form:

$$
\sum_{s=1}^{s_{T}} z_{s} m_{s}=0
$$

where the summation is over all aqueous species, $z_{s}$ is the electrical charge of a species, and $m_{s}$ is its molal concentration. The hydrogen mass balance equation can not be used as a goveming equation to calculate the $\mathrm{pH}$ from the corresponding analytical data. This is due to the impracticability if not impossibility of ever measuring the total concentration of hydrogen with sufficient accuracy when nearly all of it is contributed by the solvent. As a practical matter, even the charge balance equation can be used for this purpose only in limited circumstances.

One may associate the solvent, water, with a mass balance for oxygen. However, the mass of water in a speciation-solubility calculation is fixed at $1 \mathrm{~kg}$, and the concentration of water is entirely determined by the concentrations of the other components in the solution. Therefore, no such mass balance is required.

To sum up, the reference formulation consists of $\varepsilon_{T}-2$ mass balance equations/total concentrations (one pair for every element except oxygen and hydrogen) and the charge balance equation (to calculate $p H$ ). Each element is present in only one oxidation state. Activity coefficients are treated as known parameters.

Before proceeding, we contrast this framework (common to speciation-solubility codes in general) with that employed in the EQ6 code. In the corresponding problem in that code, we would be given $\varepsilon_{T}$ masses, in moles, and the same number of mass balance equations, this time written in terms of masses instead of concentrations. There we have a mass balance equation for oxygen, and we must calculate the mass of the solvent, water. In the case where each element appeass in only one oxidation state, as we have temporarily assumed here, the charge balance equation is a linear combination of the mass balance equations, and the governing equation associated with $\mathrm{H}^{+}$can be either a hydrogen mass balance equation or the charge balance equation. The speciation-solubility problem has one fewer unknown, hence one less governing equalion, than the corresponding EQ6 problem. 
In either the EQ3NR or EQ6 type formulation of the problem, we may formally associate one aqueous species with each balance equation; e.g., $\mathrm{Na}^{+}$with sodium balance, $\mathrm{Al}^{3+}$ with aluminum balance, and $H^{+}$with charge balance. Suppose our model must consider $n$ balance equations and $k$ aqueous complexes (using the term to include ion-pairs). That gives $k$ mass action relationships which are also governing equations. We now have $n+k$ equations in $n+k$ unknowns (the masses/concentrations/activities of the $n+k$ aqueous species).

The number of aqueous complexes is usually much greater than the number of balance equations. This is especially true when the number of balance equations becomes very large. A useful approach is to reduce the number of equations and unknowns by substituting the aqueous mass action equations into the balance equations (sec Chapter 9). This leaves us with $n$ equations (modified balance equations) in $n$ unknowns (the concentrations or activities of the aqueous species that were chosen to formally correspond to the balance relationships).

This approach leads us to the concept of dealing with a set of master aqueous species. These may also be termed basis species. However, the concept does not arise purely from an attempt to reduce the number of iteration variables. The $k$ aqueous complexes give us $k$ linearly independent dissociation reactions and $k$ linearly independent logarithmic mass action equations. An efficient way to write these reactions and equations is in terms of the associated complex (the species that dissociates) and such a set of master aqueous species. The dissociation reactions are then written as overall dissociation reactions but never as stepwise reactions; e.g., one has:

$$
\mathrm{HgCl}_{3}=\mathrm{Hg}^{2+}+3 \mathrm{Cl}
$$

not:

$$
\mathrm{HgCl}_{3}=\mathrm{HgCl}_{2}^{o}+\mathrm{Cl}^{-}
$$

We will also use this format to write dissolution reactions for minerals and gases and their associated heterogeneous mass action equations.

\subsubsection{Alternative Constraints}

The reference formulation of the aqueous speciation problem consists of:

(1) $\varepsilon_{T}-2$ mass balance equations/total concentrations.

(2) the charge balance equation (to calculate $p H$ ).

We now discuss alternative constraints to the balance equations in the reference formulation. We discuss how to put oxidation-reduction problems into the formulation in the following subsection.

The alternative constraints are:

- Specifying log activity for a species (recall $\mathrm{pH}=-\log a_{\mathrm{H}^{+}}$). 
- Log activity combination functions (e.g., $\mathrm{pHCl}$; Section 2.3.4).

- Log mean activity of an ion and one of opposite charge (Section 2.3.4).

- Applying the charge balance constraint to a master species other than $H^{+}$.

- Phase equilibrium with a pure mineral.

- Phase equilibrium with an end member of a solid solution (the composition of the solid solution must be specified).

- Phase equilibrium with a gas (the fugacity of the gas must be specified).

- Equilibrium with other aqueous species, without falling under a mass balance constraint.

- Specifying the individual concentration of an aqueous basis species.

When a mass balance constraint is replaced by one of the above, we continue to reduce the number of unknowns to a master set as discussed above. The corresponding total concentrations become parameters to be calculated. We can calculate, for example, the total mass/concentration of hydrogen. This can be done with sufficient relative accuracy to permit the EQ6 code to use it as a constraint to solve for $p H$.

The log activity constraint. The first substitution that we discuss is most often applied to the hydrogen ion. In the course of chemical analysis, the $p H$ of an aqueous solution is usually determined by means of a specific-ion electrode. This gives us the activity of the hydrogen ion from the relation:

$$
p H=-\log a_{H^{+}}
$$

The activities of many other species, including $\mathrm{Na}^{+}, \mathrm{Ca}^{2+}, \mathrm{S}^{2-}, \mathrm{F}$, and $\mathrm{Cl}$, to name but a few, may also be measured by specific-ion electrodes.

EQ3NR will accept as an input the logarithm of the activit; of a species. Note that this means that the code expects to see $-p H$, not $p H$, on the input file when this option is invoked. The new governing equation is just:

$$
m_{i}=\frac{a_{i}}{\gamma_{i}}
$$

The charge balance constraint. This can be applied to one of the major ions if a charge-balanced speciation model is desired. If EQ3NR does not use the charge balance equation as a constraint, it will calculate the charge imbalance. Otherwise, it will notify the user of the change in total concentration or $p H$ that was required to generate a charge-balanced model.

We recommend routinely calculating $p H$ from electrical balance only in cases of synthetic solutions for which the ionic totals are exact with respect to charge balance. Such solutions are most likely to be $p H$ buffer solutions. In other circumstances, this practice is potentially dangerous be- 
cause the result is affected by the error in every analytical value that is put in the model and also by every analytical value that should have been put in the model but was not. In general, apart from the case of $p H$ buffer solutions, it is only safe to calculate $p H$ this way if the $p H$ is low (high concentrations of $\mathrm{H}^{+}$) or high (high concentrations of $\mathrm{OH}$ ).

Equilibrium constraint involving a non-aqueous species. A mass balance constraint may also be replaced by an equilibrium constraint involving a specified pure mineral, solid solution component species, or gas species. Suppose we wanted to know what cencentration of dissolved calcium would be required for a water to be in equilibrium with calcite (the stable polymorph of $\mathrm{CaCO}_{3(c)}$ at $\left.25^{\circ} \mathrm{C}\right)$. The dissolution reaction may be written as:

$$
\text { Calcite }+\mathrm{H}^{+}=\mathrm{Ca}^{2+}+\mathrm{HCO}_{3}^{-}
$$

and the corresponding goveming equation is then:

$$
K_{\text {Calcile }}=\frac{a_{\mathrm{Ca}^{2+}{ }^{a} \mathrm{HCO}_{3}}}{a_{\text {Calcire }}{ }^{a_{H}}}
$$

Because calcite is a pure phase, its activity is fixed at unity.

If the required equilibrium involves an end-member component of a solid solution, the goveming equation is slightly modified. Suppose we choose equilibrium with a calcite end-member of a

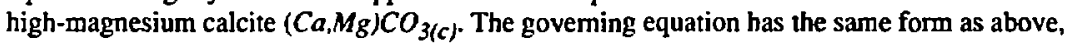
but the activity of the calcite end-member is no longer unity. Instead, one has:

$$
a_{\text {Calcite }}=\lambda_{\text {Calcile }} x_{\text {Calcite }}
$$

where $\lambda_{\text {Calcite }}$ is the activity coefficient and $x_{\text {Calcite }}$ is the mole fraction of the calcite component. The mole fraction of the $\sigma$-th component of the $\psi$-th solid solution is given by:

$$
x_{\sigma \Psi}=\frac{n_{\sigma \Psi}}{\sum_{\sigma^{*} \Psi} n_{\sigma^{\prime} \Psi}}
$$

where $n_{\sigma^{\prime} \Psi}$ is the number of moles of the $\sigma^{\prime}-$ th component and $\sigma_{T, \psi}$ is the number of such components. The current version of EQ3NR deals only with solid solutions that are composed of endmember components. The activity coefficients $\left(\lambda_{\sigma \psi}\right)$ may be computed from a variety of equations. The activity coefficient model for a given solid solution is specified on the EQ3/6 data file, which also contains the requisite supporting parameters. The formulations presently treated in EQ3/6 are discussed in Chapter 4.

Suppose we would like to know how sach dissolved carbonate would be in solution if it were in equilibrium with $\mathrm{CO}_{2(g)}$. The $\mathrm{CO}_{2(g)}$ dissolution reaction may be written as: 


$$
\mathrm{CO}_{2(g)}+\mathrm{H}_{2} \mathrm{O}_{(\mathrm{l})}=\mathrm{H}^{+}+\mathrm{HCO}_{3}^{-}
$$

The corresponding governing equation is:

$$
K_{\mathrm{CO}_{2(\mathrm{~g})}}=\frac{{ }_{\mathrm{H}^{+}} a_{\mathrm{HCO}}}{f_{\mathrm{CO}_{2}} a_{w}}
$$

Here $f_{\mathrm{CO}_{2}}$ is the fugacity of $\mathrm{CO}_{2}$. In order to use this option, the user must provide an input value for it to the speciation model.

Fugacity is a thermodynamic variable for gases that is akin to partial pressure in the same way the thermodynamic activity of an aqueous species is akin to the molal concentration. The formal relationship is given by:

$$
f_{g}=\chi_{g} p_{g}
$$

where $p_{g}$ is the parial pressure and $\chi_{g} i$ the fugacity coefficient of the $g$-th gas. The fugacity coefficient is analogous to the activity coefficient. At low pressures, it approaches unity and hence the fugacity approaches the partial pressure.

Specifying heterogeneous equilibria as inputs to an aqueous speciation model can be a bit dangerous. First, the user must choose which phases, stable or metastable, are controlling solubility equilibria. If a choice is an extremely poor one, the equilibrium concentration of a species so constrained may be very large. Furthermore, the expressions for the logarithm of the ion activity products for all such relations must be a linearly independent set in the corresponding aqueous species. (A corollary to this is that one may not constrain more than one species by the same heterogeneous equilibrium.) Such linear dependence violates the "apparent" or "mineralogic" phase rule (Wolery, 1979). This is slightly more restrictive than the phase rule of thermodynamics. Sets of equilibria that satisfy the phase rule, but only because the temperature and pressure happen to fall on a univariant curve, do not satisfy the apparent phase rule.

Equilibrium constraint involving an aqueous species. It is possible to specify equilibrium with other species in a manner in which the species so constrained does not fall under any mass balance constraints. As an example, one might treat dissolved sulfide (represented by $H S$ ) in this manner, computing it on the basis of equilibrium with sulfate and oxygen gas. The reaction relating sulfide to sulfate is:

$$
H S^{-}+2 O_{2(g)}=H^{+}+S O_{4}^{2-}
$$

The governing equation is the corresponding mass action equation:

$$
K_{H S}=\frac{{ }^{a}{ }^{+}{ }^{a} S O_{4}^{2}}{a_{H S} f_{O_{2}}^{2}}
$$


The sulfide component ( $H S$ and related species such as $\mathrm{H}_{2} \mathrm{~S}_{(\circ \mathrm{g})}$ ) does not count in the mass balance defined for sulfate. This option is similar to those involving specifying various heterogenous equilibria.

Direct specification of individual molality. EQ3NR allows input of the individual concentrations of master species. The governing equation in this case is jusi the identity:

$$
m_{i}=m_{i}
$$

It is largely appropriate only for master species that form no complexes, such as $O_{2(a q)}$ and other dissolved gases.

\subsection{4. $\mathrm{pH}$ in Brines: pHCl and Related Functions as Alternative Constraints}

Using standard methods (e.g., Bates, 1964), the pH is measured using an ion-specific electrode for the hydrogen ion in combination with a standard reference electrode (usually silver-silver chloride). The electrode pair (commonly marketed as a combination electrode) is calibrated when used by immersion in at least two standard solutions whese $p H$ values bracket the expected sample values. This method is appropriate in dilute solutions, but not in brines. The problem is the presence of a liquid junction potential in the reference electrode at the interface between the standard or sample solution and an intemal solution composed of concentrated potassium chloride. The idea behind the standard measurement is that for sufficiently dilute sample or standard solutions, the liquid junction potential will remain at an essentially constant value (which can be factored out in the calibration process). As proposed by Bates (1964), the method should be restricted to solutions of ionic strength no greater than 0.1 molal. However, it is routinely applied to more concentrated solutions, such as seawater (for which the ionic strength is nearly 0.7 molal).

The standard method fails when applied to brines because the liquid junction potential obtained with the sample is significantly changed from that obtained with the relatively dilute calibration buffers. The theory describing liquid junction potentials has been reviewed by Baes and Mesmer (1976). In general, the dependence of the liquid junction potential on the sample solution composition is complex and can not be solely related to the ionic strength. Thus, one can not simply make $p H$ measurements in the usual way using concentrated calibration standards whose ionic strengths match those of the samples. Furthermore, the theory consists of an ideal and a non-ideal part. Taking only the ideal part and making some approximations leads to the Henderson equation. This has occasionally been put forth as a means of correcting $p H$ values in concentrated solutions obtained by going through the mechanics of the standard method. This approach is highly dubious.

Recently Knauss, Wolery, and Jackson $(1990,1991)$ have proposed a method to quantify $p H$ in concentrated solutions which avoids the liquid junction potential problem by eliminating the standard reference electrode. In this method, this electrode is replaced by another specific ion electrode. If this is a chloride elecurode, what one measures is $p H C l$, which is the sum of $p H$ and $p C l$. As an input to a speciation-solubility code, this is just as adequate as the $p H$ as long as there is a separate measurement of dissolved chloride to also input. This maintains a system of $n$ equations in $n$ unknowns. The code is able to separate $p H$ from $p C l$ using an activity coefficient model 
for the dissolved species and a chosen $p H$ scale. The subject of aqueous species activity coefficients and $\mathrm{pH}$ scales is addressed in Chapter 3.

Knauss, Wolery, and Jackson (1990) used EQ3NR to compute the $\mathrm{pHCl}$ and related functions corresponding to different combinations of specific-ion electrodes of various test solutions, such as 0.01 molal $\mathrm{HCl}$ with varying concentrations of $\mathrm{NaCl}$. Pitzer's equations were used to compute the activity coefficients in these solutions, using mostly the model of Harvie, Møller, and Weare (1984) and sometimes an altemative data set given by Pitzer (1979). They then measured the corresponding electrical potentials and plotted them against the computed $\mathrm{pHCl}$ or other function. In most cases, excellent Nernstian responses were obtained, in essence identical to those one would obtain examining the standard $\mathrm{pH}$ method. This indicated that such solutions could be defined as calibration buffers. Of critical importance to constraining the $p H$ in concentrated solutions was the fact that no interference due to sodium was found in the case of the hydrogen ion electrode, even in solutions with very low hydrogen ion concentration and very high sodium ion concentration.

The only observed failures of the method involved cases in which a specific-ion electrode responded to an ion other than the one to which it was supposed to rispond. The chloride electrode was found to respond to bromide, for example. In solutions containing both bromide and chtoride, however, $\mathrm{pHBr}$ could be measured without interference by using a bromide electrode. Interferences of this type were no surprise and are in fact well known from the use of the specific ion electrodes in dilute solutions, where they are paired with a standard reference electrode.

The method appears to work, but should receive more study. There are no official recommendations or standards concerning this method, such as those which the National Institute of Standards and Technology (formerly the National Bureau of Standards) has promulgated in the case of the standard $p H$ measurement technique. One must currently make up one's own calibration buffers, which ideally should closely resemble the samples. The method has been criticized by Mesmer (1991), who prefers not to obtain $\mathrm{pH}$ by a method which requires the use of a model for the activity coefficients in the solution. He proposes alternative approaches which involve measuring the concentration of the hydrogen jon. These in turn are criticized by Knauss, Wolery, and Jackson (1991).

Values of $p H C l$ and related functions such as $p H B r$ and $p H / N a(=p H-p N a)$ can now be input to EQ3NR as alternative constraints. In the case of $p H C l$, the governing equation takes the form:

$$
\log m_{H^{+}}=-p H C l-\log \gamma_{H^{+}}-\log m_{C r}-\log \gamma_{C l}
$$

EQ3NR expects to receive input of this type in one of two general forms. The first is the activity combination parameter defined by:

$$
x_{i j}=\left|z_{j}\right| \log a_{i}-\frac{z_{i} z_{j}}{\left|z_{j}\right|} \log a_{j}
$$


This is valid for $i$ and $j$ of any charge combination. Note that ${ }_{H^{+}, C r}=-p H C l$, so it is actually $-p H C l$ that is input to the code, not $\mathrm{pHCl}$ (analogous to the input of $-\mathrm{pH}$ instead of $\mathrm{pH}$ ). The more general form of the governing equation is then:

$$
\log m_{i}=\frac{x_{i j}}{\left|z_{j}\right|}-\log \gamma_{i}+\frac{z_{i}}{z_{j}} \log m_{j}+\frac{z_{i}}{z_{j}} \log \gamma_{j}
$$

The second general form is to input the mean log activity of the electrolyte composed of ions $i$ and $j$ :

$$
\log a_{ \pm, i j}=\frac{\left|z_{j}\right| \log a_{i}+\left|z_{i}\right| \log a_{j}}{\left|z_{i}\right|+\mid z_{j}}
$$

This is not quite as general, because the two ions must have opposite signs of electrical charge. Note that $\log a_{+, H C l}=-1 / 2 p H C l$. The corresponding goveming equation is:

$$
\log m_{i}=\frac{\left|z_{i}\right|+\left|z_{j}\right|}{\left|z_{j}\right|} \log a_{ \pm, i j}-\log \gamma_{i}-\left|\frac{z_{i}}{z_{j}}\right| \log m_{j}-\left|\frac{z_{i}}{z_{j}}\right| \log \gamma_{j}
$$

\subsubsection{The Carbonate System: Dealing with Alkalinity}

To model the carbonate system, EQ3NR expects as normal input an analytical value for total dissolved bicarbonate $\left(\mathrm{CO}_{2(\mathrm{aq})}+\mathrm{HCO}_{3}{ }^{\circ}+\mathrm{CO}_{3}{ }^{2-}\right.$, where these are taken in the sense of components, including any ion pairs or complexes of the corresponding species). The appropriate measurement can be made using ion chromatography or infrared detection of carbon dioxide released from an acidified sample. The results might be expressed on a data sheet as total dissolved $\mathrm{CO}_{2}$ in $\mathrm{mg} / \mathrm{L}$. This must be converted to the equivalent concentration of $\mathrm{HCO}_{3}{ }^{-}$for input to EQ3NR, as it is defined on the supporting data files as the basis species corresponding to carbonate nass balance, This can be done by multiplying this quantity by the ratio of the molecular weight of $\mathrm{HCO}_{3}{ }^{-}(61.016 \mathrm{~g} / \mathrm{mole})$ to that of $\mathrm{CO}_{2(\mathrm{aq})}(44.009 \mathrm{~g} / \mathrm{mole})$ (the value of this ratio being 1.3864$)$. A data sheet might also list a value for "total free $\mathrm{CO}_{2}$." This represents only the $\mathrm{CO}_{2(\mathrm{aq})} \mathrm{com}$ ponent. If this is the only available measure of dissolved bicarbonate, the total dissolved bicarbonate can be computed from this and the $p H$ by inverting the relevant equations given later in this section.

Carbonate (in the form of $\mathrm{CO}_{3}{ }^{2-}$ and $\mathrm{HCO}_{3}{ }^{-}$, including any ion pairs of these species) makes up nearly all of the alkalinity of many aqueous solutions. Strictly speaking, the alkalinity is a measure of the acid neutralizing capability of an aqueous solution. However, it is also commonly used as a measure of the carbonate system. In fact, alkalinity is only an indirect measure of this system, and its usage as such a measure entails a number of assumptions which are not always valid. In this context, it is also frequently misunderstood and misused. The worst conseguence of this usage of alkalinity is that it leads people to think that a direct measurement of total dissolved bicarbonate (in the sense discussed in the above paragraph) is unnecessary. Indeed, it is common to find analytical data sheets on groundwater chemistry which lack such direct measurements. 
The purpose of this section is to discuss these problems, and to suggest means by which the total dissolved bicarbunate (in the desired sense) can be estimated, in the case in which direct measurements are lacking. These means are not always entirely satisfactory, and are here suggested mainly for the benefit of those who must work with historical data.

EQ3NR formerly allowed titration alkalinity $\left(A_{f}\right)$ to be input for bicarbonate instead of total concentration. This capability essentially matched that used in the PHREEQE code (Plummer, Parkhurst, and Thorstenson, 1980). The approach is to define an alkalinity balance equation, which is very similar to a mass balance equation. It may be written in the general form:

$$
A_{t}=\sum_{s=1}^{s_{T}} \tau_{s} m_{s}
$$

where $\tau_{s}$ is the alkalinity factor of the $s$-th species. This is the number of moles of hydrogen ion neutralized by one mole of species in the process of titrating the solution with an acid solution (usually of dilute sulfuric acid) to some end-point, usually in the range of $p H 4.0$ to 4.5 (See Standard Methods, 1976, p. 278-293; see also Plummer, Parkhurst, and Thorstenson, 1980, p. 17-18). Titration alkalinity defined in this manner is in units of equivalents per kilogram of solvent water, where "equivalent" means hydrogen ion neutralizing equivalent.

Titration alkalinity is usually not reported in these units, however. Standard Methods calls for reporting the titration alkalinity in terms of the stoichiometric equivalent of $\mathrm{mg} / \mathrm{L}$ of $\mathrm{CaCO}_{3}$. We will mark alkalinities in such units with an asterisk $\left({ }^{*}\right)$. Thus, the form of titration alkalinity usually reported must be converted according to:

$$
A_{t}=\frac{A_{t}^{*}}{50,000 \rho_{g / m l}}
$$

The " 50,000 " in the above equation is actually the product of $1000 \mathrm{mg} / \mathrm{g}$ and the molecular weight of calcium carbonate (taken as $100 \mathrm{~g} /$ mole following Standard Methods), divided by the alkalinity factor of $\mathrm{CaCO}_{3}$ (2.0 equivalents/mole). The molecular weight of $\mathrm{CaCO}_{3}$ is more accurately $100.088 \mathrm{~g} /$ mole, but the $100 \mathrm{~g} /$ mole value is used by Standard Methods in the formula for calculating $A_{t}^{*}$ from the titration data, so retaining it as above is actually more consistent with the titration measurement.

The titration alkalinity $\left(A_{t}^{*}\right)$ is referred to in Standard Methods as $T$. This quantity may appear on analytical data sheets as " $\mathrm{T}$ " or "titration alkalinity" and in units marked "mg/L" or "mg/L $\mathrm{CaCO}_{3}$." In this context, "mg/L" means "mg/L $\mathrm{CaCO}_{3}$." Users of geochemical modeling codes sometimes mistakenly interpret "mg/L" to mean that the titration alkalinity is given in units of $\mathrm{mg} / \mathrm{L} \mathrm{HCO}_{3}{ }^{-}\left(\mathrm{A}_{1, \mathrm{HCO}_{3}}\right)$. it is not unknown for analysts to report the titration alkalinity in such units as well, though this is not a standard practice. It can be obtained by multiplying $A_{t}^{*}$ by the 
molecular weight of $\mathrm{HCO}_{3}^{-}\left(61.016 \mathrm{~g} /\right.$ mole) and the alkalinity factor of $\mathrm{CaCO}_{3}$ (2.0 equivalents/mole) and dividing the result by the product of the molecular weight of $\mathrm{CaCO}_{3}$ and the alkalinity factor of $\mathrm{HCO}_{3}^{-}$(1.0 equivalents/mole). In simpler terms, one has:

$$
A_{t, \mathrm{HCO}_{3}^{-}}=1.2192 A_{t}^{*}
$$

It is very important to note that the titration alkalinity expressed in $\mathrm{mg} / \mathrm{L} \mathrm{HCO}_{3}{ }^{-}$is not equal to the total dissolved bicarbonate (in the sense required for input to EQ3NR) expressed in the same units. Recall that $\mathrm{CO}_{2(a q)}$ does not contribute to the titration alkalinity, while it does contribute to the desired total dissolved bicarbonate. Furthermore, $\mathrm{CO}_{3}{ }^{2-}$ contributes differently to the titration alkalinity (by a factor of 2 ) than it does to the desired total dissolved bicarbonate.

In the determination of alkalinity, Standard Methods calls for two end points to be determined in the titration. One of these gives $T$, the titration alkalinity, the other $P$, the phenolphthalein alkalinity. The latter corresponds to an end point of $p H$ 8.3. If the $p H$ of the sample solution is already less than or equal to this, then $P=0$. The phenolphthalein alkalinity may also appear on an analytical data sheet. Standard Methods calls for using $P$ to partition the titration alkalinity into components due to bicarbonate, carbonate, and hydroxide; thus, one may write:

$$
A_{t}^{*}=A_{\mathrm{HCO}_{3}^{-}}^{*}+A_{\mathrm{CO}_{3}^{2-}}^{*}+A_{\mathrm{OH}}^{*}
$$

This scheme implicitly assumes that no other components are present which contribute to the alkalinity. It also ignores ion pairing and complexing as it pertains to these species. Note that each of these component alkalinities is reported in units of equivalent $\mathrm{mg} / \mathrm{L} \mathrm{CaCO}$.

These three components, bicarbonate alkalinity, carbonate alkalinity, and hydroxide alkalinity, are determined from $T$ and $P$ according to the partitioning formula given in Standard Methods (1976, Table 403:I, p. 281). At least one of these three always has a value of zero. Sometimes two are zero. They are supposed to be reported in units of $\mathrm{mg} / \mathrm{L} \mathrm{CaCO}$. They are commonly found on analytical data sheets. Since they in essence determine the titration alkalinity, this quantity is sometimes omitted, and if it is desired, it must be compuled from then using the above equation.

It is not unknown for analysts to report the bicarbonate alkalinity in units of $\mathrm{mg} / \mathrm{L} \mathrm{CO}_{3}$. Users have been known to confuse the bicarbonate alkalinity expressed in such units with the total dissolved bicarbonate (the desired input to EQ3NR), which may be expressed in the same units.

The concentration of the bicarbonate component can be computed from the bicarbonate alkalinity:

$$
C_{H C O_{3}, m_{o} / L}=1.2192 \mathrm{~A}_{H C O}^{*}
$$

The numerical factor on the right hand side is the same as that appearing in eq (39). The molality of the bicarbonate component can be computed directly from the bicarbonate alkalinity: 


$$
m_{\mathrm{HCO}_{3}^{-}}=\frac{A_{\mathrm{HCO}}^{*}}{50,044}
$$

The denominator on the right hand side is the product of $1000 \mathrm{mg} / \mathrm{g}$, the molecular weight of $\mathrm{CaCO}_{3}$, and the alkalinity factor of $\mathrm{HCO}_{3}{ }^{*}$, divided by the alkalinity factor of $\mathrm{CaCO}_{3}$. The molecular weight of $\mathrm{HCO}_{3}{ }^{-}$is factored out in the derivation of this equatior.

The concentration of the carbonate component can be similarly computed from the carbonate alkalinity:

$$
C_{\mathrm{CO}_{3}^{2-}, \mathrm{mg} / \mathrm{L}}=0.5996 \mathrm{~A}_{\mathrm{CO}_{3}^{2}}^{*}
$$

The numerical factor on the right hand side is the product of the molecular weight of $\mathrm{CO}_{3}{ }^{2}$. $(60.008 \mathrm{~g} / \mathrm{mole})$ and the alkalinity factor of $\mathrm{CaCO}_{3}$, divided by the product of the molecular weight of $\mathrm{CaCO}_{3}$ and the alkalinity factor of $\mathrm{CO}_{3}^{2-}$ (also 2.0 equivalent/mole, so the alkalinity factors cancel out). The molality of the carbonate component can be computed directly from the carbonate alkalinity:

$$
m_{C O_{3}^{-}}=\frac{A_{C O_{3}^{*}}^{*}}{100,088}
$$

It is not unkiown for analysts to report the total concentration of bicarbonate as:

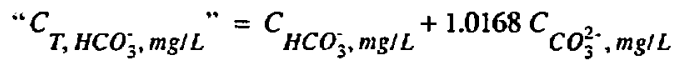

where the concentrations on the right hand side are obtained from alkalinities as above and the factor 1.0168 is the ratio of the molecular weight of $\mathrm{HCO}_{3}{ }^{-}$to that of $\mathrm{CO}_{3}{ }^{2-}$ and is used to convert the units of carbonate concentration from $\mathrm{mg} / \mathrm{L} \mathrm{CO}_{3}{ }^{2-}$ to the equivalent $\mathrm{mg} / \mathrm{L} \mathrm{HCO}_{3}{ }^{-}$. In terms of molalities, this is equivalent to taking:

$$
\text { " } m_{T_{1} \mathrm{HCO}_{3}^{-}} "=m_{\mathrm{HCO}_{3}^{-}}+m_{\mathrm{CO}_{3}^{2-}}
$$

This measure of total bicarbonate, whether repored in $\mathrm{mg} / \mathrm{L}$ or molality, is not the measure of total bicarbonate which is to be input to EQ3NR, because it does not include the contribution from the component $\mathrm{CO}_{2(a q)}$.

Above $\mathrm{pH} \mathrm{8.3,} \mathrm{the} \mathrm{contribution} \mathrm{of} \mathrm{CO}_{2(a q)}$ to total bicarbonate is negligible ( $1 \%$ or less), and estimates based on the above formulations may be input to EQ3NR with negligible error. Ai lower $p H$ values, the concentration of $C C_{2(a q)}$ can be estimated from the bicarbonate alkalinity and the pH. Standard Methods (1976, Figure 407:4, p. 297) gives a nomograph for this purpose. The nomograph ?lso takes into account the dependency on the temperature and the ionic strength, using 
the quantity "total filterable residue" as a proxy for the latter. If this procedure is followed, the total dissolved bicarbonate to be input to EQ3NR can be estimated as:

$$
C_{T, m g / L, H C O_{3}^{-}}=1.3864 C_{m g / L, C O_{2(a q)}}+C_{m g / L, H C O_{3}^{-}}+1.0168 C_{m g / L, C O}^{2-}
$$

where the factor 1.3864 is the ratio of the molecular weight of $\mathrm{HCO}_{3}^{-}$to that of $\mathrm{CO}_{2(\mathrm{aq})}(44.009$ $\mathrm{g} / \mathrm{mole}$ ). In terrss of molalitiss, this is equivalent to:

$$
m_{T, \mathrm{HCO}_{3}^{-}}={ }^{m} \mathrm{CO}_{2 a q}+m_{\mathrm{HCO}_{3}^{-}}+m_{\mathrm{CO}_{3}^{2-}}
$$

As a1: altemative to the nomograph of Standard Methods, we note that the molality of $\mathrm{CO}_{2(a q)}$ may be estimated from the molality of $\mathrm{HCO}_{3}{ }^{-}$and the $\mathrm{pH}$ by considering equilibrium for the reaction:

$$
\mathrm{CO}_{2(a g)}+\mathrm{H}_{2} \mathrm{O}_{(l)}=\mathrm{H}^{+}+\mathrm{HCO}_{3}^{-}
$$

Assuming that the activity of water differs negligibly from a value of unity, the following equation is obtained:

$$
\left.{ }^{m} \mathrm{CO}_{2(a q)}=10^{\left(-\log K_{\mathrm{CO}_{2(a q)}}+\log \gamma_{2 \mathrm{CO}_{3}^{-}}-\log \gamma_{\mathrm{CO}_{2(a q)}}-p H\right.}\right)_{\mathrm{m}_{\mathrm{HCO}}}
$$

The $\log K$ for reaction (49) has values of $-6.5804,-6.3447$, and -6.2684 at 0,25 , and $60^{\circ} \mathrm{C}$ (da ta taken from the data0.sup.R10 data file). At $25^{\circ} \mathrm{C}$, this reduces to:

$$
{ }^{m_{C O} O_{2(a q)}}=10^{6.32-p H_{m}} \mathrm{HCO}_{3}
$$

for a dilute solution of ionic strength 0.0024 molal (using the B-dot equation to calculate the activity coefficients; see Chapter 3). From this, one can see that at $\mathrm{pH} 4.33$, the molality $\mathrm{CO}_{2(\mathrm{aq})}$ is 100 times that of $\mathrm{HCO}_{3}{ }^{-}$. For seawater (ionic strength of 0.662 molal), the equation becomes:

$$
{ }^{m} \mathrm{CO}_{2(\circ q)}=10^{6.14-p H_{m}} \mathrm{HCO}_{3}
$$

One of the points that may be deduced from these equations is that alkalinity is a poor way to measure the carbonate system in waters of relatively low $\mathrm{pH}$, in which $\mathrm{CO}_{2(a q)}$ dominates the total dissolved bicarbonate (defined in the sense desired for input to EQ3NR). The propagated uncertainty in such calculations can become large owing to a contribution from the uncertainty in $\rho H$ measurement in addition to one from the uncertainty in the measurement in the titration alkalinity (which is interpreted as entirely bicarbonate alkalinity at such low $p H$ ). The propagated error is also affected to some degree by uncertainty in the values of the activity coefficients, though this is not likely to be of much significance in very dilute solutions. It is probably affected much more by contributions due to uncertainties regarding the contribution to the measured al- 
kalinity of non-carbonate species. This is a potential major problem regarding the use of alkalinity in solutions of any $p H$ value.

We have shown above how the total dissolved bicarbonate (in the sense of including aqueous carbon dioxide and carbonate) may be estimated from alkalinity measurements. These methods assume that only bicarbonate, carbonate, and hydroxide contribute to the measured alkalinity. Alkalinity can also be contributed by dissolved organic species such as acetate, by components such as borate, phosphate, silicate, and sulfide, and by some dissolved metals, such as iron and aluminum, in the form of hydroxy complexes. Of course, if one knows the concentrations of the relevant species, conections may be anempted. Such corrections cculd take the form of suburacting the estimated contributions from the measured titration alkalinity. Alternatively, one can make the corrections in a speciation-solubility calculation, using an alkalinity balance equation. It requires assigning alkalinity factors to all the relevant species. Such an approach is available in the PHREEQE code (Plummer, Parkhurst, and Thorstenson, 1980) and previous versions of EQ3NR (Wolery, 1983). Either form of correction carries various uncertainties, however, and major problems arise when the corrections are large. In using previous versions of the EQ3NR code in this way, the code has occasionally zerminated unsuccessfully because the magnitude of the corrections would have exceeded the value of the reported titration alkalinity.

Many waters of interest to geochemists have substantial amounts of alkalinity due to non-carbonate species. In oil field waters, the titration alkalinity is often heavily dominated (50-100\%) by short chain aliphatic anions, chiefly acetate, propionate, butyrate, and valerate, in order of decreasing importance (Willey et al., 1975; Carothers and Kharaka, 1978). Organic anions are also present in significant concentrations in the water in and around many landfills and other geologic waste disposal sites. These may be both products of the decomposition of organic wastes and original components of the disposed waste. Waters at disposal sites may also be rich in other components which contribute to alkalinity, such as sulfide, ammonia, phosphate, silicate, and metal hydroxy complexes. Many natural waters of interest are also high in sulfide, and a few are high in boadte.

The titration alkalinity input option was removed from EQ3NR for the following reasons:

- To avoid undue propagation of errors inherent in the method, which can be severe in cerrain cases.

- To avoid possible errors by both analysts and code users concerning the nature, interpretation, and usage of analytical data.

- To avoid the problem of having to assign alkalinity factors to new species added to the supporting data files.

- To encourage the practice of obtaining direct analytical measures of total dissolved bicarbonate.

For cases in which the code user must deal with historical data which include alkalinity measurements but no direct measures of the carbonate system, the following procedure is recommended: 
- Using the reported alkalinity and $p H$ values, estimate the total dissolved bicarbonate (total dissolved carbon dioxide expressed as bicarbonate) using the methods presented above; make rough corrections if possible for contributions of organics, sulfide, etc.

- Compute a model of the solution.

- Feed the model of the solution to the EQ6 code; simulate the titration process as described by Standard Methods (1976) and compute the corresponding value of the titration alkalinity (an example of this use of EQ6 is given in the EQ6 Theoretical Manual and User's Guide, Wolery and Daveler, 1992).

- Compare the computed value of the titration alkalinity with the reported value; if they match, stop; if not, adjust the estimate of the total dissolved bicarbonate accordingly and repeat the process until a reasonable match if obtained.

This procedure may not always work. For example, if the conuribution of bicarbonate/carbonate to the alkalinity is very small compared to that of organics, sulfide, etc., then the available data really offer no constraint on the bicarbonate/carbonate system. In such cases, the user would be wise to recognize the futility of the situation.

\subsubsection{Redox Constraints}

\subsubsection{There is No "System" Eh in Most Real Systems}

The high degree of emphasis on trying to understand the geochemistry of natural waters in terms of pure equilibrium thermodynamics has misled many people into believing that the redox state of real aqueous systems can be characterized by a single parameter, usually the $E h$ (a redox potential, given in volts). The related parameter pe, the negative of the logarithm of the hypothetical electron, is similarly incapable of describing the overall redox state of a real aqueous system.

The concept of there being such a thing as a "system" Eh or a "system" pe is based on the assumption that all redox reactions in an aqueous system are in a state of thermodynamic equilibrium. This assumption is a poor one for most real systems (Morris and Stumm, 1967; Jenne, 1981; Thorstenson, 1983; Hostetler, 1984; Lindberg and Runnells, 1984). In the rush to interpiet geochemical data by means of $E h-p H$ and pe-activity diagrams, this point is often forgotten or simply ignored. This has had the unfortunate consequence of legitimizing these variables as allencompassing redox descriptors in the minds of many students.

This misconception has no doubt been reinforced by the use of $E h$ (and sometimes pe) as inputs to speciation-solubility codes. Some of these codes require the assumption of a system Eh. Most of the better known codes, EQ3NR, WATEQ2 (Ball, Jenne, and Nordstrom, 1979), and PHREEQE (Parkhurst, Plummer, and Thorstenson, 1980) permit the use of such an input but do not require it. With sufficient analytical data, the degree of disequilibrium among various redox couples may be calculated, and the existence of a system $E h$ thus tested. Often, however, the available analytical data are insufficient to do this, and one is forced to assume a system $E h$.

Redox disequilibrium in natural aqueous systems is created by solar irradiation, radioactive decay, fluid mixing, and transfer of redox components from one phase to another. It is maintained 
primarily by the smength of covalent bonds, a major factor in the redox disequilibrium of the light elements such as carbon, hydrogen, oxygen, nitrogen, and sulfur. Biological activity literally feeds on redox disequilibrium (e.g., photosynthesis, if one counts the initial disequilibrium due to solar radiation, and chemosynthesis) and catalyzes an overall approach toward redox equilibrium.

Several well known examples of redox disequilibrium in nanural aqueous systems can be cited. One is the coexistence of dissolved oxygen and organic carbon in nearly all natural waters, implying disequilibrium between the $\mathrm{O}_{2(a q)} / \mathrm{H}_{2} \mathrm{O}_{(l)}$ couple and organic/ $\mathrm{HCO}_{3}$ couples. Another is the disequilibrium between $\mathrm{CH}_{4(\mathrm{aq})} / \mathrm{HCO}_{3}^{-}$and $\mathrm{HS}_{3} / \mathrm{SO}_{4}{ }^{2-}$ in many marine sediments (Thorstenson, 1970). A third example is the disequilibrium of $\mathrm{N}_{2(a q)} / \mathrm{NO}_{3}^{-}$with $\mathrm{O}_{2(a q} / \mathrm{H}_{2} \mathrm{O}_{(l)}$ in marine surface waters (Bemer, 1971, p. 119).

As shown below, each such couple can be treated as having have its own redox state. This can be expressed in a variety of ways, including a couple-specific $E h$ or pe. These can be calculated using the Nernst equation in conjunction with chemical analyses that are specific with respect to the two oxidation states represented in any redox couple. In the following section, we will discuss the details of this concept.

\subsubsection{Background: Redox Couples and Half-Reactions}

Oxidation-reduction in aqueous systems is commonly treated in terms of redox couples and their associated half-reactions. Common couples in aqueous solution include $\mathrm{O}_{2(a q)} / \mathrm{H}_{2} \mathrm{O}_{(\mathrm{I})}$, $\mathrm{H}_{2(\mathrm{aq})} / \mathrm{H}_{2} \mathrm{O}_{(l)}, \mathrm{Fe}^{2+} / \mathrm{Fe}^{3+}, \mathrm{HS}^{2-} / \mathrm{SO}_{4}{ }^{2-}, \mathrm{SO}_{3}{ }^{2-} / \mathrm{SO}_{4}{ }^{2-}, \mathrm{S}_{2} \mathrm{O}_{3}{ }^{2-} / \mathrm{SO}_{4}{ }^{2-}, \mathrm{NH}_{4}{ }^{+} / \mathrm{NO}_{3}{ }^{-}, \mathrm{N}_{2(\mathrm{aq})} / \mathrm{NO}_{3}{ }^{-}$, $\mathrm{CH}_{4(\mathrm{aq})} / \mathrm{HCO}_{3}$, and a host of organic/ $\mathrm{HCO}_{3}^{-}$couples. The half-reaction is illustrated in the case of the very important couple $\mathrm{O}_{2(a q)} / \mathrm{H}_{2} \mathrm{O}_{(l)}$ :

$$
2 \mathrm{H}_{2} \mathrm{O}_{(l)}=\mathrm{O}_{2(\mathrm{aq})}+4 \mathrm{H}^{+}+4 e^{-}
$$

Another very important half-seaction corresponds to the so-called hydrogen electrode:

$$
H_{2(g)}=2 H^{+}+2 e^{-}
$$

Multiplying this half-reaction by two and subtracting it from the first yields the following complete redox reaction (which has no electrons among the reactants or products):

$$
2 \mathrm{H}_{2} \mathrm{O}_{(\mathrm{l})}=\mathrm{O}_{2(\mathrm{aq})}+2 \mathrm{H}_{2(\mathrm{~g})}
$$

The thermodynamic convention used to describe the state of electrical potentials of half-reactions in terms of Eh values is to take the electrical potential of the standard hydrogen electrode as zero at all temperatures and pressures. This is consistent with the following additional thermodynamic conventions, where $\Delta G_{f, i}^{o}$ is the standard state Gibbs energy of the $i$-th species:

- $\Delta G_{f, H_{2(g)}}^{o}=0$ at all temperatures and pressures (the standard slate fugacity is 1 bar) 
- $\Delta G_{f, H^{+}}^{o}=0$ at all temperatures and pressures

- $\Delta G_{f, e^{-}}^{o}=\Delta G_{f, e^{-}}=0$ at all temperatures and pressures

The Gibbs energy $(\Delta G)$ is related to the electrical potential $(E)$ by the Nernst equation (cf. Garrels and Christ, 1965):

$$
\Delta G=+n F E
$$

where $n$ is the number of electrons in the half-reaclion and $F$ is the Faraday constant.

An alternative treatment almost equivalent to that above is to write the half-reaclions as reduction reactions, so that the electron appears on the left hand side. One then reverses the sign of the right hand side of the Nemst equation as written above. This development is equivalent to the one above, except that the signs of the Gibbs energies and corresponding equilibrium constants and activity products are reversed (Stumm and Morgan, 1981, Chapter 8).

The standard thermodynamic relation describing the Gibbs energy of reaction $\left(\Delta G_{r}\right)$,of the $r$-th reaction is:

$$
\Delta G_{r}=\Delta G_{r}^{o}+2.303 R T \operatorname{Tog} Q_{r}
$$

where $\Delta G_{r}^{o}$ is the corresponding standard state Gibbs energy of reaction and $Q_{r}$ is the corresponding activity product. Applying this to half-reactions and using the positive convention version of the Nernst equation, one obtains:

$$
E=E^{\circ}+\frac{2.303 R T}{n F} \log Q_{+, 1 / 2}
$$

where $E^{o}$ is the standard state electrical potential, and $Q_{+, / 2}$ is the activity product of the halfreaction. If one uses the negative convention version of the Nernst equation, one obtains instead:

$$
E=E^{o}-\frac{2.303 R T}{n F} \log Q_{-, 1 / 2}
$$

where $Q_{-, ~} / 2$ is the activity product of the reverse half-reaction. These relations are equivalent because $\log Q_{-, 1 / 2}=-\log Q_{+, 1 / 2}$. Because the Gibbs energy of the hypothetical electron is al. ways zero, whether it is in the standard state or not, its thermodynamic activity is fixed at unity and it need not explicitly appear in the activity product expressions for half-reactions.

\subsubsection{Background: $E h, p e, A h$, and Equilibrium Oxygen Fugacity}

One can write a modified Nernst equation for any redox couple. In the case of the ferrous-ferric couple, the corresponding half-reaction is:

$$
F e^{2+}=F e^{3+}+e^{-}
$$

The corresponding form of the Nernst equation is: 


$$
E_{\mathrm{Fe}^{2+} / \mathrm{Fe}^{3+}}=E_{\mathrm{Fe}^{2+} / \mathrm{Fe}^{3+}}^{\mathrm{o}}+\frac{2.303 R T}{F} \log \left(\frac{a_{\mathrm{Fe}^{3+}}}{a_{\mathrm{Fe}^{2+}}}\right)
$$

Under the thermodynamic conventions adopted above, the potential $E$ on the left hand side of each of the above equatior: can be taken as the $E h$ for this specific couple. This may or may not equate to the results of an " $E h$ " measurement.

If the two redox couples are in equilibrium with each other, they must have the same $E h$. Conversely, if they have the same $E h$, they must be in equilibrium. Any difference in couple-specific $E h$ values is a measure (in volts) of the degree of disequilibrium. This can be shown by relating the Gibbs energy of a combined, complete reaction to the differences in potentials. If the first half- reaction has $E h_{l}$ and $n_{l}$ electrons appear in it, and the second half- reaction has $E h_{2}$ and $n_{2}$ electrons, one can construct a complete reaction by multiplying the second half-reaction by $n_{1} / n_{2}$ and adding the result to the first half-reaction. Then $n_{l}$ electrons are transferred in the complete reaction. The Gibbs energy of this reaction is then given by:

$$
\Delta G_{r}=n_{1} F\left(E h_{1}-E h_{2}\right)
$$

The condition of zero Gibbs energy of reaction (thermodynamic equilibrium) is met if and only if $E h_{I}=E h_{2}$.

The redox parameter pe, popularized by Truesdell (1968) and Stumm and Morgan (1981), is defined to be analogous to $\mathrm{pH}$ :

$$
p e=-\log a_{e}
$$

where $e^{-}$is the hypothetical aqueous electron. It should not be confused with real aqueous electrons, which are extremely scarce in nature. Their thermodynamic properties are not the same. In fact, the hypothetical electron used to define $p e$ is not the same as the one used to define $E h$. The $E / h$ conventions require the activity of the hypothetical electron to always be unity. That convention would fix pe at a value of zero.

The relation between pe and $E h$ is:

$$
p e=\left(\frac{F}{2.303 R T}\right) E h
$$

(Thorstenson, 1970; Stumm and Morgan, 1981). One may derive that this requires the thermodynamic convention

- $\Delta G_{f, e^{-}}=\frac{1}{2} \Delta G_{H_{2(g)}}-\Delta G_{H}$ at all temperatures and pressures

This in turn requires that

- $\Delta G_{f, e^{-}}=0$ at all temperatures and pressures

whereas :he Eh convention for the hypothetical electron was 
- $\Delta G_{f, e^{-}}^{o}=\Delta G_{f, e^{-}}=0$ at all temperatures and pressures

It should be clear that $p e$ is not a perfect analog to $p H$, because $p H$ is defined with respect to $\mathrm{H}^{+}$. a real aqueous species, whereas pe is defined with retpect to a hypothetical species. Each redox couple can have its own $p e$, just as it can have its owil $E h$, the two being related by the equation given above. It follows from the previous development that thermodynamic equilibrium between two redox couples is synonymous with each having the same value of pe.

The state of an aqueous redox couple can also be expressed in terms of chemical affinity by the redox affinity, $A h$ (Wolery, 1983). This is a special case of the thermodynamic affinity function (its application to half-reactions). It is related to $E h$ by the relation

$$
A h=F E h
$$

The driving force for any kind of complete chemical reaction (meaning to exclude half-reactions) can be expressed by the thermodynamic affinity $\left(A_{r}\right)$, which is related to the equilibrium constant $K_{r}$ and the activity product $Q_{r}$ by the equation:

$$
A_{r}=-2.303 R T \log \left(\frac{Q_{r}}{K_{r}}\right)
$$

If $n_{1}$ electrons appear in one half-reaction and $n_{2}$ in another, the two half-reactions can be combined into a complete redox reaction in the manner discussed previously. The thermodynamic affinity of the complete reaction, in which $n_{I}$ electrons are transferred, is then related to the $A h$ values $\left(A h_{l}\right.$ and $A h_{2}$, respectively) of the two half reactions $b_{j}$ the equation:

$$
A_{r}=n_{1}\left(A h_{1}-A h_{2}\right)
$$

Thermodynamic equilibrium $(A=0)$ among two redox couples is the case if and only if both couples have the same value of $A h$.

Alternatively, the state of a redox couple may be expressed in terms of an equilibrium oxygen fugacity (a couple-specific oxygen fugacity). Fugacities are properties of gas species. Gas species do not exist in aqueous solution because, by definition, all species in aqueous solution are aqueous species. Therefore, we can only talk about oxygen fugacities in aqueous solution by reference to hypothetical equilibria with a gas phase. Putting it another way, $O_{2(g)}$ makes a perfectly good hypothetical aqueous species, much like the hypothetical aqqueous electron.

Consider the half-reaction:

$$
2 \mathrm{H}_{2} \mathrm{O}_{(l)}=\mathrm{O}_{2(\mathrm{~g})}+4 \mathrm{H}^{+}+4 \mathrm{e}^{-}
$$

where we now take $\mathrm{O}_{2(g)}$ to be a hypothetical acjueous species with the thermodynamic properties of the real gas species. We can calculate an equilibrium oxygen fugacity for any half-reaction by coupling it with this one to form a complete redox reaction. 
Let $K_{E h}$ be the equilibrium constant for the $\mathrm{O}_{2(g)} / \mathrm{H}_{2} \mathrm{O}_{(l)}$ half- reaction given above. Eh and oxygen fugacity are then related by the equation:

$$
\log f_{O_{2}}=\left(\frac{4 F}{2.303 R T}\right) E h-4 \log a_{H^{+}}+2 \log a_{w}+\log K_{E h}
$$

This equation can be used to relate the equilibrium oxygen fugacity of any redox couple with its own Eh. Two redox couples are in thermodynamic equilibrium with each other if and only if they have the same equilibrium oxygen fugacity.

\subsubsection{Redox Options: Testing versus Assuming Equilibrium}

A commonly used approach in aqueous speciation modeling is to input a total concentration for a dissolved element that occurs in more than one oxidation state and partition it according to a given $E h$, pe, or oxygen fugacity. This, however, requires us to assume that all redox couples in the system are in a state of thermodynamic equilibrium. The EQ3NR code offers this option.

If we constrain the thermodynamic activities of all the aqueous species appearing in a couple's half-reaction withoul resorting to an input $E h, A h, p e$, or oxygen fugacity, the equations presented above give us a means to calculate its individual redox state expressed as any of the following:

- Eh (in terms of an electrical potential).

- Ah (in terms of a chemical potential).

- pe.

- oxygen fugacity.

Analytical techniques dc not generally discriminate between a simple species and its ion-pairs and complexes. However, there are techniques in many cases to discriminate between different oxidation states. To calculate the $E h$ of the ferrous-ferric couple, for example, we must have analytical data for both $\mathrm{Fe}^{2+}$ and $\mathrm{Fe}^{3+}$ (see for example Nordstrom, Jenne, and Ball, 1979). If these data are both total concentrations (e.g, total $\mathrm{Fe}^{3+}$, total $\mathrm{Fe}^{3+}$ ), we simply have two mass balance equations for iron in the aqueous speciation model instead of one.

This is the preferred approach for treating oxidation-reduction in aqueous speciation modeling (see Nordstrom et al., 1979). One may then test whether or not various redox couples are in equilibrium with each other. EQ3NR can treat any redox couple in this fashion. Alternative constraints discussed in the previous subsection could substitute for one or both total concentrations/ mass balances in the usual way. The code will use a redox default to partition an element that appears in more than one oxidation state if insufficient data are input to calculate a couple-specific parameter. The redox default may be an input $E h$, a pe, or log oxygen fugacity. Altematively, it may be defined by a redox couple for which sufficient data are input to calculate couple-specific parameters. By constraining one or more of the species in the corresponding half-reaction by a heterogeneous equilibrium constraint, it is possible to constrain the default redox state by a heterogeneous equilibrium. 


\subsubsection{Measures of Mineral Saturation.}

EQ3NR employs two measures of the saturation state of an aquecus solution with respect to minerals. The first is the saturation index defined as:

$$
S I=\log \frac{Q}{K}
$$

where it is understood that $Q$ is the activity product and $K$ the equilibrium constant for a dissolution reaction. In the case of the dissolution of calcite, for example, if the reaction is written as:

$$
\text { Calcite }+\mathrm{H}^{+}=\mathrm{Ca}^{2+}+\mathrm{HCO}_{3}^{-}
$$

the ion activity product is then defined as:

$$
Q_{\text {Calcire }}=\frac{a_{C a^{2+} a_{H C O}}}{a_{\text {Calcite }} a_{H^{+}}}
$$

The second measure of the saturation state is the thermodynamic affinity of the precipitation reaction. The affinity of a reaction (no matter how it is written) is related to its activity product and equilibrium constant by:

$$
A=-2.303 R T \log \frac{K}{Q}=2.303 R T \log \frac{Q}{K}
$$

Because $\log Q / K$ reverses sign when the reaction is reversed, the affinity to precipitate is related to the saturation index by:

$$
\text { A. }=2.303 R T S I
$$

Following these conventions, both $S I$ and $A_{-}$are positive for supersaturated minerals, zero for saturated ones, and negative for undersaturated minerals.

In the case of solid solution minerals with end-member components, the saturation index of the $\sigma$-th end member is related to that of the corresponding pure phase $\phi$ by:

$$
S I_{\sigma \Psi}=S I_{\phi}-\log a_{\sigma \psi}=S I_{\phi}-\log x_{\alpha \Psi}-\log \lambda \sigma \psi
$$

where $a_{\sigma \psi}$ is the thermodynamic activity of the end-member, $x_{\sigma \psi}$ is its mole fraction, and $\lambda_{\sigma \psi}$ is its mole fraction activity coefficient. Consideration of an overall dissolution reaction of a solid solution of given composition suggests that the saturation index of the $\psi$-th solid solution should be defined by:

$$
S I_{\psi}=\sum_{\sigma=1}^{\sigma r_{\psi} \psi} x_{\sigma \psi} S I_{\sigma \psi}
$$


Affinity functions can be defined analogously.

The problem of defining the saturation state of a solid solution for which no composition is given is not so straightforward, because the result is composition-dependent. One way to approach this would be to find the compositions that maximize the $S I$. This is the method presently employed in EQ3/6 (see Bourcier, 1985, 1989).

\subsection{Use and Misuse of Speciation-Solubility Codes}

There is significint potential to misuse any speciation-solubility code. No such code should be used as a "black box". As Jenne (1981, p. 36) puts it, "... each application should be viewed as a partial villidation." The geochemicial model of each new scenario (e.g., a set of waters in a compositional range not previously studied) nuay have a different set of important aqueous species, and hente provide a test of some thermodynamic data that have not previously been exercised. Also, reactions controlled by equilibrium in one situation may be in disequilibrium in another, and vice versa, especially heterogeneous and aqueous redox reactions.

Geochemicial modeling with acueous speciattion-solubility codes must actively address three questions. First, are all the significant species in the model? Second, are all the important thermodynamic data sufficiently correct? Do they make sense when compared with the model outputs when working with a set of wa u amples? Do they make sense in comparison with other knowledge about in aqueous systen . ach as data on the identities of minerals with which the water is in contact? Third, would disecuilibrium constraints be more appropriate than equilibrium constraints for sone reactions (especially aqueous redox reactions)? Users should keep in mind the :admonition of Nordstrom et al. (1979) that "... no model is better than the assumptions on which it is based."

If no thermodynannic data are available for species known or suspected to be important in a given application, then such data should be estimated by empirical or semi-empirical methods. EQ3NR has an input file option which permits the user to temporarily modify equilibrium constants at run time (see Chapter 6). This makes it convenient to conduct sensitivity studies of the uncertainty in such estimated values. Langmuir (1979) summarizes approaches for estimating thermodynamic properties of agueous species and teactions. Tardy and Garrels (1974), Wolery (1978), and Helgeson et al. (1978) discuss methods for estimating the thermodynamic properties of minerals.

A common problem faced by novices at speciation-solubility modeling is that their models come out grossly supersaturated with ne:uly every aluminum and ferric iron bearing mineral in the data base. This often occurs because analysis is made of inadequately filtered samples, which commonly contain colloidal particles of these two components. These particles then "inflate" the corresponding chemical analyses. Busenbery (1978) showed that large quantities of a colloidal aluninum phase occurred in the size range $0.1-0.45 \mu \mathrm{m}$ during a set of feldspar dissolution experimer.is. Latwen and Chandler (19R2) did more detailed studies of iron particulate size distribution in fresh waters. Their work shows that a filter finer than $0.1 \mu \mathrm{m}$ is necessary to effectively remove these particulates from the chemical analysis.

The modeler should be awatre that many solubility-controlling phases, especially at low temperature, are metastable (e.g., atmorphous $\mathrm{F}_{\mathrm{e}}(\mathrm{OH})_{3}$ may control the ievel of dissolved iron, not the 
more stable hematite, $\mathrm{Fe}_{2} \mathrm{O}_{3}$ ). In addition, the stability of some controlling phases may be somewhat variable due to such factors as crystallinity (i.e., crystal size), order/disorder, ionic substitution, or, in the case of fresh precipitates, aging. Helgeson et al. (1978) discuss many of these effects.

One approach that may be helpful to users is to estimate the amount of aluminum or iron that would be in solution under the assumption of appropriate solubility equilibria. For example, one might constrain dissolved aluminum to satisfy equilibrium with gibbsite $\left.(\mathrm{Al} / \mathrm{OH})_{3(\mathrm{C})}\right)$ or constrain iron to satisfy equilibrium with amorphous $\mathrm{Fe}(\mathrm{OH})_{3}$ or a nontronite (ferric-rich smectile) clay. However, this is not a substitute for analysis of carefully filtered samples.

The state of available analytical data on water compositions is often a limiting factor in the usage of EQ3NR or any other speciation-solubility code. In general, the data must be both accurate and sufficiently complete. Inaccurate data often result when methods suitable for analyzing drinking water are applied to waters very dissimilar to this medium. This carn take the form of both positive and negative interferences. Some analytical pitrameters (c.g., $p H$, alkalinity, dissolved sulfide) must be measured immediately upon sampling to avoid changes due to mineral precipitation, ingassing, or outgassing. Water samples should be inspected after transportation and storage for the formation of precipitates. Quite often, analytical data are incomplete for geochemical modeling purposes. This may have the effect of completely inhibiting modeling work, or it may result in modeling with assumptions that could have been avoided if the right hard data had been available. In general, analytical work is most useful to modeling if there is interplay between the modeler and the analyst.

Internal consistency can provide useful tests of the quality of aqueous speciation models (see Merino, 1979). One such test is to compare the calculated electrical imbaiance with the cation/anion subtotals for charge equivalents. EQ3NR makes these calculations, which are a meaningful test if electrical balance is not used as an input constraint. Merino (1979) also recommends the technique of comparing measured and independently calculated values of titration alkalinity. In essence, his recommendation corresponds to the currently recommended procedure for dealing with alkalinity described earlier in this chapter. 


\section{Activity Coefficients of Aqueous Species}

\subsection{Introduction}

The thermodynamic activities $\left(a_{i}\right)$ of aqueous solute species are usually defined on the basis of molalities. Thus, they can be described by the product of their molal concentrations $\left(m_{i}\right)$ and their molal activity coefficients $\left(\gamma_{i}\right)$ :

$$
a_{i}=m_{i} \gamma_{i}
$$

The thermodynamic activity of the water $\left(a_{w}\right)$ is always defined on a mole fraction basis. Thus, it cun be described analogously by product of the mole fraction of water $\left(x_{w}\right)$ and its mole fraction activity coefficient $\left(\lambda_{s v}\right)$ :

$$
a_{w}=x_{w} \lambda_{s}
$$

It is also possible to describe the thermodynamic activities of aqueous solutes on a mole fraction basis. However, such mole fraction-based activities $\left(a_{i}^{(x)}\right)$ are not the same as the more familiar molality-based activities $\left(a_{i}{ }^{(m)}\right)$, as they are defined with respect to different choices of standard states. Mole fraction based activities and activity coefficients $\left(\lambda_{i}\right)$, are occasionally applied to aqueous nonelectrolyte species, such as ethanol in water. In geochemistry, the aqueous solutions of interest almost always contain electrolytes, so mole-fraction based activities and activity coefficients of solute speries are little more than theoretical curiosities. In EQ3/6, only molalitybased activities and activity coefficients are used for such species, so $a_{i}$ always implies $a_{i}^{(m)}$. Becaluse of the nature of molality, it is not possible to define the activity and activity coefficient of water on a molal basis; thus, $a_{w}$ always means $a_{w}(x)$.

Solution thermodynamics is a construct designed to approximate reality in terms of deviations from some defined ideal bchavior. The complex dependency of the activities on solution composition is thus dealt with by shifting the problem to one of describing the activity coefficients. The usual treatment of aqueous solutions is one which simultaneously employs quantities derived from, and therefore belonging to, two distinct models of ideality (Wolery, 1990). All solute activity coefficients are based on solality and have unit value in the corresponding model of ideality, called molality-based ideality. The activity and activity coefficient of water are not constant in an ideal solution of this type, though they do approach unit value at infinite dilution. These solvent properties are derived from mole fraction-based ideality, in which the mole fraction activity coefficients of all species enmponents in solution have unit value. In an ideal solution of this type, the molal activity coefficients of the solutes are not unity, though they approach it at infinite dilution (see Wolery, 1990).

Any geochemical modeling code which treats atueous solutions must provide one or more models by which to compute the activity coefficients of the solute species and the solvent. In many codes, what is computed is the set of $\gamma_{i}$ plus $a_{w}$. As many of the older such icoles were constructed to deal only with dilute solutions in which the atcivity of water is no less than about 0.98 , some of these just take the activity of water to be unity. With the advent of activity coefficient models 
of practical usage in concentrated solutions (mostly based on Pitzer's 1973, 1975 equations), there has been a movement away from this particular and severe approximation. Nevertheless, it is generally the activity of water, rather than the activity coefficient of water, which is evaluated from the model equations. This is what was previously done in EQ3/6. However, EQ3/6 now evaluates the set of $\gamma_{j}$ plus $\lambda_{1,}$. This is done to avoid possible computational singularities that may arise, for example if heterogeneous equilibria happen to fix the activity of water (e.g., when a solution is saturated with both gypsum and anhydrite).

Good models for activity coefficients must be accurate. A prerequisite for general accuracy is thermodynamic consistency. The activity coefficient of each aqueous species is not independent of that of any of the others. Each is related to a corresponding partial derivative of the excess Gibbs energy of the solution $\left(G^{E X}\right)$. The excess Gibbs energy is the difference between the complete Gibbs energy and the ideal Gibbs energy. Because there are two models of ideality, hence two models for the ideal Gibbs energy, there are two forms of the excess Gibbs energy, $G^{E X m}$ (molality-based) and $G^{E X x}$ (mole fraction-based). The consequences of this are discussed by Wolery (1990). In version 7.0 of EQ3/6, all activity coefficient models are based on ideality defined in terms of molality. Thus, the excess Gibbs energy of concern is $G^{E X m}$. The activity of water, which is based on mole-fraction idenlity, is imported into this structure as discussed by Wolery (1990). The relevant differential equations are:

$$
\begin{gathered}
\ln \gamma_{i}=\frac{1}{R T} \frac{\partial G^{E X m}}{\partial n_{i}} \\
\ln a_{w}=-\frac{\Sigma m}{\Omega}+\frac{1}{R T} \frac{\partial G^{E X m}}{\partial n_{w}}
\end{gathered}
$$

where $R$ is the gas constant, $T$ the absolute temperature, $\Omega$ the number of moles of solvent water comprising a mass of $1 \mathrm{~kg}(\Omega \approx 55.51)$, and:

$$
\Sigma m=\sum_{i} m_{i}
$$

the sum of molalities of all solute species. Given an expression for the excess Gibbs energy, such equations give a guaranteed route to thermodynamically consistent results (Pitzer, 1984; Wolery, 1990). Equations that are derived by other routes may be tested for consistency using other relations, such as the following forms of the cross-differentiation rule (Wolery, 1990):

$$
\begin{gathered}
\frac{\partial \ln \gamma_{j}}{\partial m_{i}}=\frac{\partial \ln \gamma_{i}}{\partial m_{j}} \\
\frac{\partial \ln a_{w}}{\partial n_{i}}=\frac{\partial \ln \gamma_{i}}{\partial n_{w}}-\frac{1}{n_{w}}
\end{gathered}
$$


In general, such equations are most easily used to prove that a set of model equations is not thermodynamically consistent. The issue of sufficiency in proving consistency using these and related equations (Gibbs-Duhem equations and sum nules) is addressed by Wolery (1990).

The activity coefficients in reality are complex functions of the composition of the aqueous solution. In electrolyte solutions, the activity coefficients are influenced mainly by electrical interactions. Much of their behavior can be correlated in terms of the ionic strength, defined by:

$$
I=\frac{1}{2} \sum_{i} m_{i} z_{i}^{2}
$$

where the summation is over all aquecous solute species and $z_{i}$ is the electrical charge. However, the use of the ionic strength as a means of correlating and predicting activity coefficients has been taken to unrealistic extremes (c. . , in the mean salt method of Garrels and Christ, 1965, p. 58$60)$. In general, model equations which express the dependence of activity coefficients on solution composition only in terms ot the ionic strength are restricted in applicability to dilute solutions.

The three basic options for computing the activity coefficients of aqueous species in EQ3/6 are models based respectively on the Davies (1962) equation, the "B-dot" equation of Helgeson (1969), and Pitzer 's $(1973,1975,1979,1987)$ equations. The first two models, owing to limitations on accuracy, are only usefti in dilute solutions (up to ionic strengths of 1 molal at most). The third basic model is useful ir: highly concentrated as well as dilute solutions, but is limited in terms of the components that can be treated.

With regard to temperature and p.essure dependence, all of the following models are parameterized along the $1 \mathrm{~atm} / \mathrm{steam}$ saturation curve. This corresponds to the way in which the temperature and pressure dependence of standard state thermodynamic data are also presently treated in the software. The pressure is thus a function of the temperature rather than an independent variable, being fixed at 1.013 bau fro: $0-100^{\circ} \mathrm{C}$ and the pressure for steam/liquid water equilibrium from $100-300^{\circ} \mathrm{C}$. However, somc of the data files have more limited temperature ranges.

\subsection{The Davies Equation}

The first activity coefficient mod 31 in EQ3/6 is based on the Davies (1962) equation:

$$
\log \gamma_{i}=-A_{\gamma, 10} z_{i}^{2}\left(\frac{\sqrt{I}}{1+\sqrt{I}}+0.2 I\right)
$$

(the constant 0.2 is sometimes also taken as 0.3 ). This is a simple extended Debye-Hückel model (it reduces to a simple Debye-Huickel model if the " $0.2 \Gamma$ " part is removed). The Davies equation is frejuently used in geochemice modeling (e.g., Parkhurst, Plummer, and Thorstenson, 1980; Stumm and Morgan, 1981). Note that it expresses all dependence on the solution composition through the ionic strength. Also, he activity coefficient is given in terms of the batse ten logatrithm, instead of the natual logiathm. The Debye-Hückel $A_{\gamma}$ parameter bears the additional label " 10 " to ensure consistency with this. The Davies equation is normally only used for temperatures close to $25^{\circ} \mathrm{C}$. It is only accurate up to ionic strengths of a few tenths molal in most 
solutions. In some solutions, inaccurac $y$, defined as the condition of model results differing from experimental measurements by more than the experimental error, is apparent at even lower concentrations.

In EQ3/6, the Davies equation option is selected by selting the option flag jopg1 =-1. A supparting data file consistent with the use of a simple extended Debye-Hückel model must also be supplied (e.g., datal = data1.com, data1.sup, or datal.nea). If iopg $1=-1$ and the supporting data file is not of the appropriate type, the software terminates with an error message.

The Davies equation has one great strength: the only species-specific parameter required is the electrical charge. This equation may therefore readily be applied to a wide spectrum of species, both those whose existence is well-established and those whose existence is only hypothetical.

The Davies equation predicts a unit activity coefficieat for all neutral solute species. This is known to be inaccurate. In general, the activicy coefficients of neutral species that are non-polar

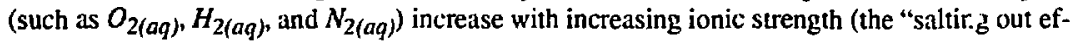
fect," so named in reference to the corresponding decreasing solubilities of such species as the salt concentration is increased; cf. Garrels and Christ, 1965, p. 67-70). In addition, Reardon and Langmuir (1976) have shown that the activity coefficienis of two polar neutral species (the ion pairs $\mathrm{CaSO}_{4(\mathrm{aq})}$ and $\mathrm{MgSO}_{4(\mathrm{ag})}$ ) decrease with increasing ionic strength, presumably as a consequence of dipole-ion interactions.

The Davies equation is thermodynamically consistent. It is easy to show, for $\mathrm{e}_{\alpha}$ umple, that it satisfies the solute-solute form of the cross-differentiation equation.

Most computer codes using the Davies equation set the activity of water to one of the following: unity, the mole fraction of water, or a limiting expression for the mole fraction of water. Usage of any of these violates thermodynamic consistency, but this is probably not of great significance as the inconsistency is numerically not significant at the relatively low concentratioris at which the Davies equation itself is accurate. For usage in EQ3/6, we have used standard thermodynamic relations to derive the following expression:

$$
\log a_{w}=\frac{1}{\Omega}\left(-\frac{\Sigma m}{2.303}+\frac{2}{3} A_{\gamma, 10^{I}} I^{\frac{3}{2}} \sigma(\sqrt{I})-2(0.2) A_{\gamma, 10} I^{2}\right)
$$

where " 2.303 " is a symbol for and approximation of $\ln 10$ (warning: this is not in general a sufficiently accurate approximation) and:

$$
\sigma(x)=\frac{3}{x^{3}}\left(1+x-\frac{1}{1+x}-2 \ln (1+x)\right)
$$

This result is thermodynamically consistent with the Davies equation.

\subsection{The B-dot Equation}

The second model for activity coefficients available in $E Q 3 / 6$ is based on the $B$-dot equation of Helgeson (1969) for eleritrically charged species: 


$$
\log \gamma_{i}=-\frac{A_{\gamma, 10} z_{i}^{2} \sqrt{I}}{1+\mathfrak{a}_{i} B_{\gamma} \sqrt{I}}+\dot{B} I
$$

Here $a_{i}$ is the hard core diameter of the species, $B_{y}$ is the Debye-Hückel $B$ parameter, and $\dot{B}$ is the characteristic B-dot parameter. Like the Davies equation, this is a simple extended DebyeHückel model, the extension being the " $\dot{B} I$ " term. The Debye-Hūckel part of this equation is equivalent to that of the Davies equation if the product " $a_{i} B_{\gamma}$ " has a value of unity. In the extended part, these equations differ in that the Davies equation has a coefficient in place of $\dot{B}$ which depends on the electrical charge of the species in question.

In EQ3/6, the B-dot equation option is selected by setting the option flag iopg $1=0$. A supporting data file consistent with the use of a simple extended Debye-Hückel model must also be supplied (e.g., datal = datal.com, datal.sup, or datal.nea). Note that these data files support the use of the Davies equation as well (the $?_{j}$ data on these files is simply ignored in that case). If iopgl = 0 and the supporting data file is n.st of the appropriate type. the software terminates with an error message.

The B-dot equation has about the same level of accuracy as the Davies equation, and almost as much universality (one needs to know $a_{j}$ in addition to $z_{j}$ ). However, it fails to satisfy the solutesolute form of the cross-differentiation nule. The first term is consistent with this rule only if all hard core diameters have the same value. The second is consistent only if all ions share the same value of the square of the electrical charge. However, the numerical significance of the inconsistency is small in the range of low concentrations in which this equation can be applied with useful accuracy. On the positive side, the B-(lot equation has been developed (Helgeson, 1969) to span a wide range of temperature (up to $300^{\circ} \mathrm{C}$ ).

For electrically neutral solute species, the B-dot equation teduces to:

$$
\log \gamma_{i}=\dot{B} l
$$

As $\dot{B}$ has positive values at all temperatures in the range of application, the equation predicts a salting out effect. However, by tradition (Helgeson et al., 1970), the B-dot equation itself is not used in the case of neutral solute species. The practice, as suggested by Garrels and Thompson (1962) and reiterated by Helgeson (1969), is to assign the value of the activity coefficient of aqueous $\mathrm{CO}_{2}$ in otherwise pure sodium chloride solutions of the same ionic strength. This function was represented in previous versions of $E Q 3 / 6$ by a power series in the ionic strength:

$$
\log \gamma_{i}=k_{1} l+k_{2} l^{2}+k_{3} I^{3}+k_{4} I^{4}
$$

The first term on the right hand side dominates the others. The first coefficient is positive, so the atrivity coefficient of $\mathrm{CO}_{2}$ increatses with increasing ionic strength (consistent with the "salting out" effect). As it was applied in EQ3/6, the coefficients for the power series themselves were represented as similar power series in temperature, and this model was fit to data taken from Ta- 
ble 2 of Helgeson (1969). These data (including extrapolations made by Helgeson) covered the range $25-300^{\circ} \mathrm{C}$ and $0-3$ molal $\mathrm{NaCl}$.

The high order power series in eq (90) was unfortunately very unstable when extrapolated outside the range of the data to which it was fit. EQ3NR and EQ6 would occasionally run into an unrecoverable problem attempting to evaluate this model for high ionic strength values generated in the process of attempting to find a numerical solution (not necessarily because the solutions in question really had high ionic strength). To eliminate this problem, the high order power series has been replaced by a new expression after Drummond (1981, p. 19):

$$
\ln \gamma_{i}=\left(C+F T+\frac{G}{T}\right) J-(E+H T)\left(\frac{J}{I+1}\right)
$$

where $T$ is the absolute temperature and $\mathrm{C}=-1.0312, \mathrm{~F}=0.0012806, \mathrm{G}=255.9, \mathrm{E}=0.4445$, and $H=-0.001606$. Note that this is presented in terms of the natural logarithm. Conversion is accomplished by using the relation:

$$
\log x=\frac{\ln x}{2.303}
$$

This expression is both much simpler (considering the dependencies on both temperature and ionic strength) and is more stable. However, in deriving it, the ionic strength was taken to be equivalent to the sodium chloride molality. In the original model (based on Helgeson, 1969), the ionic strength was based on correcting the sodium chloride molality for ion pairing. This correction is numerically insignificant at low temperature. It does become significant at high temperature. However, neither this expression nor the power series formulation it replaced is thermodynamically consistent with the B-dot equation itself, as can be shown by applying the solute-solute cross-differentiation rule.

The more recent previous versions of $\mathrm{EQ} 3 / 6$ only applied the " $\mathrm{CO}_{2}$ " approximation to species that are essentially nonpolar (e.g., $\mathrm{O}_{2(a q)}, \mathrm{H}_{2(\mathrm{aq})}, \mathrm{N}_{2(\mathrm{aq})}$ ), for which salting-out would be expected. In the case of polar neutral aqueous species, the activity coefficients were set to unity (following the recommendation of Garrels and Christ, 1965, p. 70); i.e., one has:

$$
\log \gamma_{i}=0
$$

This practice is still followed in the present version of the code.

EQ3/6 formerly complemented the B-dot equation with an approximation for the activity of water that was based on assigning values in pure sodium chloride solutions of the same "stoichiometric" ionic strength (Helgeson et al., 1970). This approximation was fairly complex and was, of course, not thermodynamically consistent with the B-dot equation itself. In order to simplify the data requir 'ments, as well as avoid the need to employ a second ionic strength function, this formulation has been replaced by a new one which depends on the $\hat{B}$ parameter and is quasi-consistent with the B-dot equation: 


$$
\log n_{w}=\frac{1}{\Omega}\left(-\frac{\sum m}{2.303}+\frac{2}{3} A_{\gamma, 10} I^{\frac{3}{2}} \sigma\left(a B_{\gamma} \sqrt{I}\right)-\dot{B} I^{2}\right)
$$

The solute hard core diameter (a) is assigned a fixed value of $4.0 \AA$ (a reasonable value). This equation is consistent with the B-dot equation if all solute species are ions, have the same fixed value of the hard core diameter, and have the same value of the square of the electrical charge.

\subsection{Scaling of Individual Ionic Activity Coefficients: pH Scales}

Before proceeding to a discussion of Pitzer's $(1973,1975)$ equations, we will address the problem of scaling associated with the activity coefficients of individual ions. It is not possible to observe (measure) any of the thermodynamic functions of such species, because any real solution must be electrically balanced. Thus, the activity coefficients of aqueous ions can only be measured in electrically neutral combinations. These are usually expressed as the mean activity coefficients of neutral electrolytes. The mean activity coefficient of neutral electrolyte $M X(M$ denoting the cation, $X$ the anion) is given by:

$$
\log \gamma_{ \pm, M X}=\frac{v_{M} \log \gamma_{M}+v_{X} \log \gamma_{X}}{v_{M X}}
$$

where $v_{M}$ is the number of moles of cation produced by dissociation of one mole of the electrolyte, $v_{X}$ is the number of moles of anion produced, and:

$$
v_{M X}=v_{M}+v_{X}
$$

Electrical neutrality requires that:

$$
z_{M}{ }^{v}=-z^{v}{ }^{v}
$$

Although the activity coefficients of ions can not be individually observed, the corresponding molal concentrations can be. The corresponding products, the thermodynamic activities of the ions, are not individually observable, precisely because of the problem with the activity coefficients. Thus, the problem of obtaining individual activity coefficients of the ions and the problem of obtaining individual activities of the same species is really the same problem.

Individual ionic activity coefficients can be defined on a conventional basis by introducing some arbitrary choice. This is can be made by adopting some expression for the activity coefficient of a single ion. The activity coefficients of all other ions then follow via electroneutrality relations. The activities for all the ions are then also determined (cf. Bates and Alfenaar, 1969). Because this applies to the hydrogen ion, such an arbitrary choice then determines the $p H$. Such conventions are usually made precisely for this purpose, and they are generally known as $p H$ scales. The NBS $p H$ scale, which is the basis of nearly all modem conventional $p H$ measurement, is based on the Bates-Guggenheim equation (Bates, 1964): 


$$
\log \gamma_{C t}=\frac{-A_{\gamma, 10} \sqrt{I}}{1+1.5 \sqrt{I}}
$$

This scale is significant not only to the measurement of $p H$, but of corresponding quantities (e.g." $p C l, p B r, p N a$ ) obtained using other specific-ion electrodes (cf. Bates and Alfenaar, 1969; Bates, 1973; Bates and Robinson, 1974).

The Bates-Guggenheim equation, like the Davies equation and the B-dot equation, is an extended Debye-Hückel formula. However, if one applies the Davies equation or the B-dot equation to the chloride ion, the result is not precisely identical. The difference approaches zero as the ionic strength approaches zero, and is not very significant quantitatively in the low range of ionic strength in which either the Davies equation or the B-dot equation has useful accuracy. Nevertheless, the use of either of these equations in uncorrected form introduces an inconsistency with measured $p H$ values, as use of the Davies equation for example would interpret the $p H$ as being on an implied "Davies" scale.

Activity coefficients (and activities) of ions can be moved from one scale to another. The general relation for converting from scale (1) to scale (2) is (Knauss, Wolery, and Jackson, 1991):

$$
\log \gamma_{i}^{(2)}=\log y_{i}^{(1)}=\frac{z_{i}}{z_{j}}\left(\log \gamma_{j}^{(2)}-\log \gamma_{j}^{(1)}\right)
$$

For example, if we evaluate the Davies equation for all ions, we may take the results as being on scale (1). To convert these to the NBS scale (here scale (2)), we take the $j$-th ion to be the chloride ion and evaluate the Bates-Guggenheim equation. We then apply the scale conversion equation to every other ion $i$.

In $E Q 3 / 6$, activity coefficients are first calculated from the "raw" single-ion equations. They are then immediately rescaled, unless no rescaling is to be done. Thus, rescaling occurs during the iteration process; it is not deferred until convergence has been achieved. The user has control over rescaling via the option switch iopg2. If iopg $2=0$, all single-ion activity coefficients and activities are put on the NBS scale. If $\operatorname{iopg} 2=-1$, no rescaling is performed. If $\operatorname{iopg} 2=1$, all $\sin$ gle-ion activity coefficients and activities are put on a scale which is defined by the relation:

$$
\log \gamma_{H^{+}}=0
$$

This has the effect of making the activity and the molality of the hydrogen ion numerically equal. This may have some advantages in comparing with experimental measurements of the hydrogen ion molality. Such measurement technịues have recently been discussed by Mesmer (1991).

The problem of scaling the atcivity coefficients of ions is more acute in concentrated solutions, and the need to discriminate among different scales in geochemical modeling codes has only been addressed as such codes have been written or modified to treat such solutions (e.g., Harvie, Maller, and Weare, 1984; Plummer et al., 1988). 


\subsection{Pitzer's Equations}

\subsubsection{Introduction}

Pitzer (1973, 1975) proposed a set of semi-empirical equations to describe activity coefficients in aqueous electrolytes. These equations have proven to be highly successful as a means of dealing with the thermodynamics of concentrated solutions (e.g.. Pitzer and Kim, 1974). Models based on these equations have been developed to describe not only solution properties, but also equilibrium between such solutions and salt minerals (e.g., Harvie and Weare, 1980; Harvie, Moller, and Weare, 1984). The utility of these models in geochemical studies has been well established. For example, such models have been shown to account for the mineral sequences produced by evaporation of seawater (Harvie et al., 1980), the process of trona deposition in Lake Magadi, Kenya (Monnin and Schott, 1984), and the formation of the borate-rich evaporite deposits at Searles Lake, California (Felmy and Weare, 1986).

Pitzer's equations are based on a semi-theoretical (sce Pitzer, 1973) interpretation of ionic interactions, and are written in terms of interaction coefficients (and parameters from which such coefficients are calculated). There are two main categories of such coefficients, "primitive" ones which appear in the original theoretical equations, but most of which are only observable in certain combinations, and others which are "observable" by virtue of corresponding to observable combinations of the primitive coefficients or by virtue of certain arbitrary conventions. Only the observable coefficients are reported in the literature.

There is a very extensive literature dealing with Pitzer's equations and their application in both interpretation of experimental data and calculational modeling. A complete review is beyond the scope of the present manual. Discussion here will be limited to the equations themselves, how to use them in $\mathrm{EQ} 3 / 6$, and certain salient points that are necessary in order to use them in an informed manner. Readers who wish to pursue the subject further are referred to reviews given by Pitzer (1979, 1987, 1992). Jackson (1988) has addressed the verification of the addition of Pitzer's equations to EQ3/6.

In EQ3/6, the Pitzer's equations option is selected by setting the option flag iopgl $=1$. A supporting data file consistent with this option must also be supplied (e.g., datal $=\mathbf{d a t a l . h m w ~ o r ~}$ datal.pit). If iopg $1=1$ and the supporting data file is not of the appropriate type, the software terminates with an error message.

Pitzer's equations are based on the following virial expansion for the excess Gibbs energy:

$$
G^{E X m}=R T\left(w_{w w} f(l)+\left(\frac{1}{w_{w}}\right) \sum_{i j} \lambda_{i j}(I) n_{i} n_{j}+\left(\frac{1}{w_{w}^{2}}\right) \sum_{i j k} \mu_{i j k} n_{i} n_{j} n_{k}\right)
$$

where $w_{w}$ is the number of kilograms of solvent water; $f(l)$ is a Debye-Hückel function describing the long-range electrical interactions to first order, the subscripts $i, j$, and $k$ denote aqueous solute species, and $n_{i}$ is the number of moles of the $i$-th solute species. The equation also contains two kinds of interaction or virial coefficients: the $\lambda_{i j}$ are second order interaction coefficients, and the $\mu_{i j k}$ are third order interaction coefficients. A key element in the success of Pitzer's equations is 
the treatment of the second order interaction coefficients as functions of ionic strength. As will be discussed later in more detail, the $\lambda_{i j}$ consist of both theoretically defined and empirical parts, while the $\mu_{i j k}$ are completely empirical. As the term is used here, "empirical" means that at least some of the parameter values required to cvaluate a quantity must be obtained by fitting experimental data.

The sums in the interaction coefficient terms are actually double and triple sums. As the number of components in a system increases, the number of interaction coefficients of the type shown above becomes very !arge. It tums out there are many more of the $\lambda_{i j}$ and $\mu_{i j k}$ than can actually be observed, other than in combination. For example, in the equation for the excess Gibbs energy, it is quickly obvious that $\lambda_{12}$ and $\lambda_{21}$ can only be seen in the combination $\left(\lambda_{12}+\lambda_{2 l}\right)$, and a similar situation holds in the case of the $\mu_{i j k}$. This leads to the first simplification in dealing with these coefficients, which is that those with the same subscripts (regardless of order or permutation) are required to be equal (Pitzer, 1973). This is not the end of the story, as other constraints (mostly related to electrical neuratity) force even more simpliffentions (which will be addressed lateri.

A set of thermodynamically consistent equations for the activity coefficients follows by application of the partial differential equations given previously. In the case of solute species, this leads to:

$$
\ln \gamma_{i}=\left(\frac{z_{i}^{2}}{2}\right) f^{\prime}(I)+2 \sum_{j} \lambda_{i j}(I) m_{j}+\sum_{j k}\left(\left(\frac{z_{j}^{2}}{2}\right) \lambda_{j k}^{\prime}(I)+3 \mu_{i j k}\right) m_{j^{\prime} k_{k}}
$$

where $f(I)$ is the derivative $d f f l I$ and $\lambda_{i j}^{\prime}(I)$ is similarly $d \lambda_{i j} f d I$. For water, the corresponding result is:

$$
\begin{gathered}
\ln a_{w}=-\frac{\sum m}{\Omega}-\frac{1}{\Omega}\left(I f^{\prime}(I)-f(I)\right) \\
+\frac{1}{\Omega}\left(\sum_{i j}\left(\lambda_{i j}(I)+\lambda_{j i}^{\prime}(I)\right) m_{i} m_{j}+2 \sum_{i j k} \mu_{i j k} m_{i} m_{j} m_{k}\right)
\end{gathered}
$$

The activity of water is closely related to the osmotic coefficient $(\phi)$ :

$$
\ln a_{w}=-\left(\frac{\Sigma m}{\Omega}\right) \phi
$$

The thermodynamic properties of water are often discussed in the physical chemistry literature in terms of the osmotic coefficient instead of the activity of water (or the mole fraction activity coefficient of water).

The Debye-Hückel model used in Pitzer's equations is not the usual Debye-Hückel-charging formulation exemplified in the Davies or B-dot equations, but a different one derived by Pitzer (1973) and called the Debye-Hückel-osmotic model. The relevant equations are: 


$$
\begin{gathered}
f(I)=-\left(\frac{4 A_{\phi} I}{b}\right) \ln (1+b \sqrt{I}) \\
f^{\prime}(I)=-2 A_{\phi}\left(\frac{2}{b} \ln (1+b \sqrt{I})+\frac{\sqrt{I}}{(1+b \sqrt{I})}\right)
\end{gathered}
$$

The Debye-Hückel parameter $A_{\phi}$ is related to the more familiar $A_{\gamma_{1} 10}$ by:

$$
A_{\phi}=\frac{2.303 A_{y, 10}}{3}
$$

The parameter $b$ is assigned a constant value of 1.2 (Pitzer, 1973). Theoretically, this is the product $\stackrel{a}{a} B_{\dot{\gamma}}$; thus the hard core diameter at $25^{\circ} \mathrm{C}$ is effectively fixed at a value of about $3.65 \AA$ (and somewhat different values at other temperatures). Differences in the hard core diameters of various ions in solution are not explicitiy accounted for (this is the case also in the Davies equation). However, the interaction coefficient terms of the equation effectively compensate for this. A very important feature of the Debye-Hückel-osmotic model is that it, like the Debye-Hückel-charging model, is consistent with the Debye-Hückel liniting law:

$$
\log \gamma_{i} \rightarrow-A_{y, 10} z_{i}^{2} \sqrt{I} \text { as } I \rightarrow 0
$$

\subsection{Solutions of Electrolytes}

In a pure solution of aqueous neutral electrolyte $M X$, the following combinations of interaction coefficients are observable:

$$
\begin{gathered}
B_{M X}(I)=\lambda_{M X}(I)+\left|\frac{z_{X}}{2 z_{M}}\right| \lambda_{M M}(I)+\left|\frac{z_{M}}{2 z_{X}}\right| \lambda_{X X}(I) \\
C_{M X}^{\phi}=3\left(\left|\frac{z_{X}}{z_{M}}\right|^{\frac{1}{2}} \mu_{M M X}+\left|\frac{z_{M}}{z_{X}}\right|^{\frac{1}{2}} \mu_{M X X}\right)
\end{gathered}
$$

For example, the osmotic coefficient for such a solution can be written in the form (Fitzer, 1973):

$$
\begin{gathered}
\phi-1=\frac{\left|z_{M} z_{X}\right|}{2}\left(I f^{\prime}(I)-f(I)\right) \\
+\left(\frac{2 v_{M} v_{X}}{v_{M X}}\right) B_{M X}^{\phi}(I) m_{M X}+\left(\frac{2\left(v_{M} v_{X}\right)^{\frac{3}{2}}}{v_{M X}}\right) C_{M X^{m^{2}}}^{2}
\end{gathered}
$$

Appearing in this equation is $B_{M X}^{\phi}$, which is given by: 


$$
B_{M X}^{\phi}(I)=B_{M X}(I)+I B^{\prime}{ }_{M X}(I)
$$

Here $B_{M X}^{\prime}(I)$ is the derivative of $B_{M X}(I)$ with respect to the ionic strength.

The ionic strength dependence of $B_{M X}^{\phi}$ was defined by Pitzer (1973) to take the following form:

$$
B_{M X}^{\phi}(I)=\beta_{M X}^{(0)}+\beta_{M X}^{(1)} e^{-\alpha \sqrt{I}}
$$

where $\alpha$ was assigned a constant value of 2.0. $\beta_{M X}^{(0)}$ and $\beta_{M X}^{(1)}$, along with $C_{M X}^{\phi}$, are parameters whose values are determined by fitting experimental data, such as for the osmotic coefficient. Corresponding to the above equation is:

$$
B_{M X}(I)=\beta_{M X}^{(0)}+\beta_{M X}^{(1)} g(\alpha \sqrt{l})
$$

where:

$$
g(x)=\left(\frac{2}{x^{2}}\right)\left(1-(1+x) e^{-x}\right)
$$

Pitzer and Mayorga (1974) proposed a description for $B_{M X}^{\phi}$ in the case of 2:2 electrolytes that is based on an additional fitting parameter:

$$
B_{M X}^{\phi}(I)=\beta_{M X}^{(0)}+\beta_{M X}^{(1)} e^{-\alpha_{1} \sqrt{I}}+\beta_{M X}^{(2)} e^{-\alpha_{2} \sqrt{I}}
$$

Here $\alpha_{J}$ is assigned a value of 1.4 and $\alpha_{2}$ one of 12.0 and $\beta_{M X}^{(2)}$ is the additional fitting parameter. Corresponding to this is:

$$
B_{M X}(I)=\beta_{M X}^{(0)}+\beta_{M X}^{(1)} g\left(\alpha_{1} \sqrt{I}\right)+\beta_{M X}^{(2)} g\left(\alpha_{2} \sqrt{I}\right)
$$

We consider first the exponential function in eqs (113) and (117). This is shown in Figure 2 for the three commonly used values of $\alpha$. At zero ionic strength, this function has a value of unity. Thus, $B_{M X}^{\phi}=\beta_{M X}^{(0)}+\beta_{M X}^{(1)}$ or $B_{M X}^{\phi}=\beta_{M X}^{(0)}+\beta_{M X}^{(1)}+\beta_{M X}^{(2)}$. The magnitude of each term containing $\beta_{M X}^{(1)}$ or $\beta_{M X}^{(2)}$ decreases exponentially as the ionic strength increases, approaching zero as the ionic strength approaches infinity (a limit which is not of physical interest). Most of the decay takes place in the very low ionic strength range. Thus, the terms in $\beta_{M X}^{(1)}$ and $\beta_{M X}^{(2)}$ are important parts of the model, even in dilute solutions. 


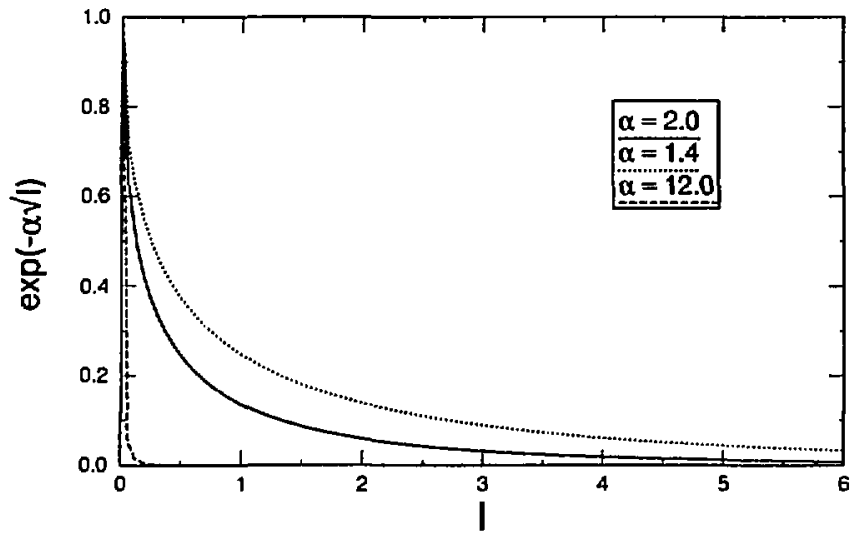

Figur 2. Behavior of the exponential function governing the ionic strength dependence of second-order interactions among cations and anions.

The function $g(x)$ is shown in Figure 3 for the three commonly used $\alpha$ values. It resembles the above exponential function, though it does not decay quite so rapidly. This function may be expanded as follows:

$$
g(x)=1-2\left(\frac{2 x}{3 !}-\frac{3 x}{4 !}+\frac{4 x^{2}}{5 !}-\frac{5 x^{3}}{6 !}+\ldots\right)
$$

This shows that $g(x)=1$ at $x=0(I=0)$. Thus, at zero ionic strength, $B_{M X}=\beta_{M X}^{(0)}+\beta_{M X}^{(1)}$ or $B_{M X}=\beta_{M X}^{(0)}+\beta_{M X}^{(1)}+\beta_{M X}^{(2)}$. It can be shown that $g(x)$ approaches zero as $x$ (and $I$ ) approach infinity.

The development thus far shows that there are two major categories of interaction coefficients. The $\lambda_{i j}$ and the $\mu_{i j k}$ in terms of which the theoretical equations were originally derived are what we will call the primitive interaction coefficients. The observable combinations of these, such as $\beta_{M X}^{(0)}, \beta_{M X}^{(1)}, \beta_{M X}^{(2)}$, and $C_{M X}^{\phi}$, are what we will call the observable interaction coefficients. This latter kind of interaction coefficient represents the model data that are reported for the various systems for which Pitzer's equations have been fit to experimental data. 


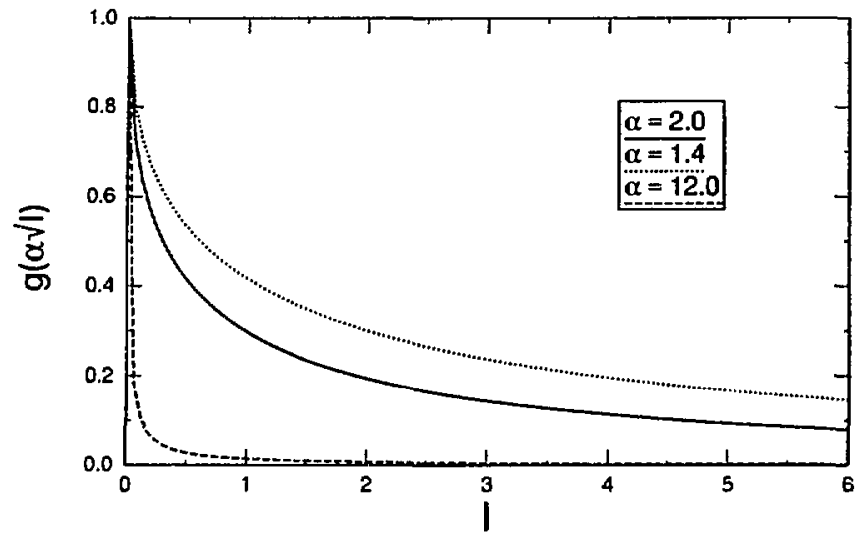

Figure 3. Behavior of the $g(x)$ function governing the ionic strength dependence of second-order irteractions among cations and anions.

It is possible to rewrite the equations for $\ln \gamma_{i}$ and $\ln a_{w}$ in complex mixtures in terms of the observable interaction coefficients. An example of such equations was suggested by Pitzer (1979) and adopted with changes in notation by Harvie, Moller, and Weare (1984). These equations are much more complex than the original form written in terms of the primitive interaction coefficients. They have been incorporated into computer codes, such as that of Harvie, Meller, and Weare (1984), PHRQPITZ (Plummer et al., 1988), and SOLMINEQ.88 (Perkins et al., 1990). As noted in the previous section, there is no unique way to construct equations for single-ion activity coefficients. Furthermore, direct usage of such equations constitutes implicit adoption of a corresponding $p H$ scale. In the case of the single-ion activity coefficient equation suggested by Pitzer, this could be termed the "Pitzer" scale.

The equations for $\ln \gamma_{i}$ and $\ln a_{v}$ which are evaluated in EQ3/6 are those written in terms of the primitive interaction coefficients. The set of these which is used is not the generalized theoretical set, which is not obtainable for the reasons discussed previously, but a practical set that is obtained by mapping the set of reported observable interaction coefficients using a set of equations that contain arbitrary conventions. These mapping equations imply a $\mathrm{pH}$ scale. We will show that the conventions chosen here match those suggested by Pitzer (1979), so this implied $p H$ scale is identical to his.

The basic guides to choosing such mapping conventions are pleasing symmetries and the desirability of minimizing the number of conventional primitive interaction coefficients with non-zero values. In the case of the second order coefficients, both of these considerations suggest the following definitions: 


$$
\begin{gathered}
\lambda_{M M}(I)=0 \\
\lambda_{X X}(I)=0 \\
\lambda_{M X}(I)=B_{M X}(I)
\end{gathered}
$$

Analogous to the formulas used to describe $B_{M X}$, one may write:

$$
\lambda_{M X}(l)=\lambda_{M X}^{(0)}+\lambda_{M X}^{(1)} g(\alpha \sqrt{I)}
$$

or:

$$
\lambda_{M X}(I)=\lambda_{M X}^{(0)}+\lambda_{M X}^{(1)} g\left(\alpha_{1} \sqrt{l}\right)+\lambda_{M X}^{(2)} g\left(\alpha_{2} \sqrt{I}\right)
$$

From the principle of corresponding terms, it follows that the corresponding mapping equations are:

$$
\begin{array}{cc}
\lambda_{M M}^{(n)}=0 & \text { for } n=0,2 \\
\lambda_{X X}^{(n)}=0 & \text { for } n=0,2 \\
\lambda_{M X}^{(n)}=\beta_{M X}^{(n)} & \text { for } n=0,2
\end{array}
$$

Evaluation of the equations for $\ln \gamma_{i}$ and $\ln a_{w}$ also requires the ionic strength derivatives of the $\lambda_{i j}$ coefficients. These are given by:

$$
\lambda_{M X}^{\prime}(I)=\lambda_{M X}^{(1)} g^{\prime}(x)\left(\frac{\alpha}{2 \sqrt{I}}\right)
$$

or:

$$
\lambda_{M X}^{\prime}(I)=\lambda_{M X}^{(1)} g^{\prime}(x)\left(\frac{\alpha_{1}}{2 \sqrt{I}}\right)+\lambda_{M X}^{(2)} g^{\prime}(x)\left(\frac{\alpha_{2}}{2 \sqrt{I}}\right)
$$

where $g^{\prime}(x)$ is the derivative of $g(x)$ (with respect to $x$, not $I$ ), given by

$$
g^{\prime}(x)=-\left(\frac{4}{x^{3}}\right)\left(1-e^{-x}\left(1+x+\frac{x^{2}}{2}\right)\right)
$$

The principle of pleasing symmetry suggests the following mapping equations for dealing with the $C_{M X}^{\phi}$ parameter: 


$$
\begin{gathered}
\mu_{M M X}=\frac{1}{6}\left|\frac{z_{M}}{z_{X}}\right|^{\frac{1}{2}} C_{M X}^{\phi} \\
\mu_{M X X}=\frac{1}{6}\left|\frac{z_{X}}{z_{M}}\right|^{\frac{1}{2}} C_{M X}^{\phi}
\end{gathered}
$$

The two $\mu$ coefficients are then related by:

$$
\frac{\mu_{M M X}}{z_{M}}=\frac{\mu_{M X X}}{\left|z_{X}\right|}
$$

These are in fact the mapping equations used in EQ3/6. However, the principle of minimizing the number of conventional primitive interaction coefficients would suggest instead mapping relations such as:

$$
\begin{gathered}
\mu_{M M X}=\frac{1}{3}\left|\frac{z_{M}}{z_{X}}\right|^{\frac{1}{2}} C_{M X}^{\phi} \\
\mu_{M X X}=0
\end{gathered}
$$

Note that with this set of mapping relations, a different $p H$ scale would be implied.

In mixtures of aqueous electrolytes with a common ion, two additional observable combinations of interaction coefficients appear (Pitzer, 1973; Pitzer and Kim, 1974):

$$
\theta_{M M^{\prime}}(I)=\lambda_{M M^{*}}(I)-\left(\frac{z_{M^{*}}}{2 z_{M}}\right) \lambda_{M M^{\prime}}(I)-\left(\frac{z_{M}}{2 z_{M^{\prime}}}\right) \lambda_{M^{\prime} M^{\prime}}(I)
$$

and:

$$
\Psi_{M M^{\prime} X}=6 \mu_{M M^{\prime} X}-\left(\frac{3 z_{M}}{z_{M}}\right) \mu_{M M X}-\left(\frac{3 z_{M}}{z_{M^{\prime}}}\right) \mu_{M^{\prime} M^{\prime} X}
$$

Here $M$ and $M$ are two cations and $X$ is the anion, or $M$ and $M^{n}$ are two anions and $X$ is the cation. From previously adopted mapping conventions, it immediately follows that the corresponding mappings are given by:

$$
\begin{gathered}
\lambda_{M M^{\prime}}(I)=\theta_{M M^{\prime}}(l) \\
\mu_{M M^{\prime} X^{\prime}}=\frac{1}{6}\left(\Psi_{M M^{\prime} X^{\prime}}+\left(\frac{3 z_{M^{\prime}}}{z_{M}}\right) \mu_{M M X}+\left(\frac{3 z_{M}}{z_{M^{\prime}}}\right) \mu_{M^{\prime} M^{\prime} X}\right)
\end{gathered}
$$


In the original formulation of Pitzer's equations (Pitzer, 1973), the $\theta_{M M}$ ' coefficient is treated as a constant. It was later modified by Pitzer (1975) to take the following form:

$$
\theta_{M M^{\prime}}(I)=s_{\theta_{M M^{\prime}}}+E_{\theta_{M M^{\prime}}(I)}
$$

$\theta_{M M^{\prime}}(I)$ corresponds to the $\Phi_{i j}$ of Harvie, Maller, and Weare (1984). The first term is a constant and accounts for short-range effects (this is the $\theta_{i j}$ of Harvie, Møller, and Weare). The second term, which is the newer part, is entirely theoretical in nature and accounts for higher-order electrostatic effects. Only the ${ }^{s} \theta_{M M^{\prime}}$ part is obtained by fitting. Corresponding to this is the equation:

$$
\lambda_{M M^{\prime}}(I)=s_{\lambda_{M M^{\prime \prime}}}+E_{\lambda_{M M^{\prime}}(I)}
$$

The relevant mapping relation is then:

$$
s_{\lambda_{M M^{\prime}}}=s_{\theta_{M M^{\prime}}}
$$

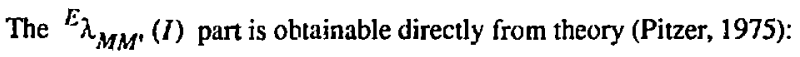

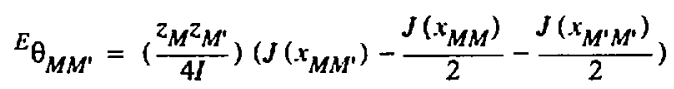

where:

$$
J(x)=\frac{1}{x} \int_{0}^{\infty}\left(1+q+\frac{q^{2}}{2}-e^{q}\right) y^{2} d y
$$

in which:

$$
q=-\left(\frac{x}{y}\right) e^{-y}
$$

and:

$$
x_{i j}=6 z_{i} z_{j} A^{\phi} \sqrt{I}
$$

The derivative of ${ }^{E_{\lambda_{M M^{\prime}}}}(I)$ is given by: 


$$
\begin{gathered}
E_{\lambda_{M M^{\prime}}^{\prime}}(I)=-\left(\frac{E_{\lambda(I)}}{I}\right) \\
+\left(\frac{z_{M^{2} M^{\prime}}}{8 I^{2}}\right)\left(x_{M M^{\prime}} J^{\prime}\left(x_{M M^{\prime}}\right)-\frac{x_{M M^{\prime}}{ }^{\prime \prime}\left(x_{M M^{\prime}}\right)}{2}-\frac{x_{M^{\prime} M^{\prime}} J^{\prime}\left(x_{M^{\prime} M^{\prime}}\right)}{2}\right)
\end{gathered}
$$

Expansion of $J(x)$ gives (Pitzer, 1975):

$$
J(x)=-\left(\frac{x^{2}}{6}\right)(\ln x+0.419711)+\ldots
$$

Application of L'Hospital's rule shows that $J(x)$ goes to zero as $x$ goes to zero (hence also as the ionic strength goes to zero). $J(x)$ is a monotonically increasing function. So is $J^{\prime}(x)$, which approaches a limiting value of 0.25 as $x$ goes to infinity. The function $J(x)$ and its derivative are approximated in EQ3/6 by a Chebyshev polynomial method suggested by Harvie and Weare (1980). This method is described in detail by Harvie (1981, Appendix $B$, in which $J(x)$ is referred to as $J_{0}(x)$ ); this method is also described in the review by $\operatorname{Pitzer}(1987$, p. 131-132).

Pitzer (1979) showed that substitution of the observable interaction coefficients into the singleion activity coefficient equation gives the following result for cation $M$ :

$$
\begin{gathered}
\ln \gamma_{M}=z_{M^{2}}^{2} f^{\gamma}+2 \sum_{a} m_{a}\left[B_{M a}+\left(\sum m z\right) C_{M a}\right]+ \\
2 \sum_{c} m_{c} \theta_{M c}+\sum_{c} \sum_{a} m_{c} m_{a}\left[z_{M}^{2} B_{c a}^{\prime}+z_{M} C_{c a}+\Psi_{M c a^{\prime}}\right]+ \\
\frac{1}{2} \sum_{a} \sum_{a^{\prime}} m_{a^{\prime}} m_{a^{\prime}}\left[z_{M^{2} \theta_{a a^{\prime}}}+\Psi_{M a a^{\prime}}\right]+\frac{z_{M}^{2}}{2} \sum_{c} \sum_{c^{\prime}} m_{c} m_{c^{\prime}} \theta_{c c^{\prime}}+ \\
z_{M}\left\{\sum_{c} \frac{m_{c} \lambda_{c c}}{z_{c}}-\sum_{a} \frac{m_{a^{\prime}} \lambda_{a a}}{\left|z_{a}\right|}+\frac{3}{2} \sum_{c} \sum_{a} m_{c} m_{a}\left(\frac{\mu_{c c a}}{z_{c}}-\frac{\mu_{c a a}}{\left|z_{a}\right|}\right)\right\}
\end{gathered}
$$

Here $a$ denotes anions, $c$ denotes cations, and:

$$
\begin{gathered}
f^{\gamma}=\frac{f^{\prime}}{2} \\
C_{M X}=\frac{C_{M X}^{\phi}}{2 \sqrt{\left|z_{M}^{2} X\right|}} \\
\Sigma m z=\sum_{c} m_{c} m_{c}
\end{gathered}
$$


(The single-ion equation for an anion is analogous). As pointed out by Pitzer, the unobservability of single-ion activity coefficients in his model lies entirely in the last term (the fourth line) of the equation and involves the primitive interaction coefficients $\lambda_{c c}, \lambda_{a a}, \mu_{c c a}$, and $\mu_{c a a}$. His suggested conventional single-ion activity coefficient equation is obtained by omitting this part. This requires the affected primitive interaction coefficients to be treated exactly as in the previously adopted mapping equations. This approach could in fact have been used to derive them.

In theory, the relevant data required to evaluate Pitzer's equations for complex mixtures of relatively strong aqueous electrolytes can all be obtained from measurements of the properties of pure aqueous electrolytes (giving the observable interaction coefficients $\beta_{M X}^{(0)}, \beta_{M X}^{(1)}, \beta_{M X}^{(2)}$, and $C_{M X}^{\phi}$ ) and mixtures of two aqueous electrolytes having a common ion ( $s_{\theta_{M M}}$ and $\Psi_{M M}$ ). There is one peculiarity in this fitting scheme in that $s_{\theta_{M M}}$ is obtainable from more than one mixture of two electrolytes having a common ion, because this parameter does not in theory depend on that ion. Thus, the value adopted may have to be arrived at by simultaneously considering the experimental data for a suite of such mixtures.

\subsubsection{Solutions of Electrolytes and Nonelectrolytes}

In general, it is necessary to consider the case of solutions containing nonelectrolyte solute species in addition to ionic species. Examples of such uncharged species include molecular species such as $\mathrm{O}_{2(a q)}, \mathrm{CO}_{2(a q)}, \mathrm{CH}_{4(a q)}, \mathrm{H}_{2} \mathrm{~S}_{(a q)}, \mathrm{C}_{2} \mathrm{H}_{5} \mathrm{OH}_{(a q)}$, and $\mathrm{SiO}_{2(a q)}$; strongly bound complexes, such as $\mathrm{HgCl}_{3(a q)}$ and $\mathrm{UO}_{2} \mathrm{CO}_{3(a q)}$; and weakly bound ion pairs such as $\mathrm{CaCO}_{3(a q)}$ and $\mathrm{CaSO}_{4(\mathrm{aq})}$. The theoretical treatment of these kinds of uncharged species is basically the same. There are practical differences, however, in fitting the models to experimental data. This is simplest for the case of molecular neutral species. In the case of complexes or ion pairs, the models are complicated by the addition of corresponding mass action equations.

The treatment of solutions of electrolytes using Pitzer's equations is quite standardized. In such solutions, there is one generally accepted relation for describing single-ion activity coefficients, though it may be expressed in various equivalent forms. Thus, in such solutions there is only one implied "Pitzer" $\rho H$ scale. Also, the set of parameters to be obtained by regressing experimental measurements is well established. Unfortunately, this is not the case for the treatment of solutions containing both electrolytes and nonelectrolytes.

Harvie, Møller, and Weare (1984) used Pitzer's equations to construct a model of all of the major components of seawater at $25^{\circ} \mathrm{C}$. They modified the equations for electrolyte systems to include some provision for neutral species-ion interactions. Additional modification was made by Felmy and Weare (1986), who extended the Harvie, Moller, and Weare model to include borate as a component. The Felmy and Weare equation for the activity of water (obtained from their equation for the osmotic coefficient) is: 


$$
\begin{aligned}
\ln a_{t v}= & -\frac{\Sigma m}{\Omega}-\frac{2}{\Omega}\left(\frac{I f^{\prime}-f}{2}+\sum_{c} \sum_{a} m_{c^{\prime}} m_{a}\left(B_{c a}^{\phi}+Z C_{c a}\right)\right. \\
& +\sum_{c} \sum_{c^{\prime}>c} m_{c} m_{c^{\prime}}\left(\Phi_{c c^{\prime}}^{\phi}+\sum_{a} m_{a} \Psi_{a a^{\prime} c}\right) \\
& +\sum_{a} \sum_{a^{\prime}>a} m_{a} m_{a^{\prime}}\left(\Phi_{a a^{\prime}}^{\phi}+\sum_{c} m_{c} \Psi_{c c^{\prime} a^{\prime}}\right) \\
& \left.+\sum_{n} \sum_{c} m_{n m_{c} \lambda_{n c}}+\sum_{n} \sum_{a} m_{n} m_{a} \lambda_{n a}+\sum_{n} \sum_{c} \sum_{a} m_{n} m_{c} m_{a} \zeta_{n c a}\right)
\end{aligned}
$$

In this equation, $c$ denotes a cation and $a$ an anion, and the following definitions are introduced:

$$
\begin{gathered}
Z=\sum_{i}\left|z_{j}\right| m_{i} \\
\Phi_{i j}^{\phi}=s_{\theta_{i j}}+E_{\theta_{i j}}(I)+I^{E} \theta_{i j}^{\prime}(I)
\end{gathered}
$$

The first three lines are equivalent to the mixture formulation given by Pitzer (1979). The fourth line (last three terms) is the new part. Here $n$ denotes a neutral species, $\lambda_{n c}$ and $\lambda_{n e}$ are second order interaction coefficients describing neutral species-ion interactions, and $\zeta_{n c a}$ is an observable third order coefficient. These new interaction coefficients are treated as constants. The terms in $\lambda_{n c}$ and $\lambda_{n a}$ were introduced by Harvie, Mbller, and Weare (1984) to treat the species $\mathrm{CO}_{2(a q) \text {. }}$ The term in $\zeta_{n c a}$ was put in by Felmy and Weare (1986) and is a third order interaction coefficient. It was necessary to include it in the equations to account for interactions involving the species $B(O H)_{3(a q)}$.

The corresponding singlc-ion equation for cation $M$ takes the following form:

$$
\begin{gathered}
\ln \gamma_{M}=z_{M}^{2} F+\sum_{a} m_{a}\left[2 B_{M a}+Z C_{M a}\right] \\
+\sum_{c} m_{c}\left(2 \Phi_{M c}+\sum_{a} m_{a} \Psi_{M c a}\right) \\
+\sum_{a} \sum_{a^{\prime}>a} m_{a^{\prime} m_{a^{\prime}}} \Psi_{M a a^{\prime}}+\left|z_{M}\right| \sum_{c} \sum_{a} m_{c} m_{a} C_{c a} \\
+2 \sum_{n} m_{n} \lambda_{n M}+\sum_{n} \sum_{a} m_{n} m_{a} \zeta_{n a M}
\end{gathered}
$$


Here $\Phi_{i j}$ is the $\theta_{i j}$ of the earlier notation and:

$$
\begin{gathered}
F=\frac{f^{\prime}}{2}+\sum_{c} \sum_{a} m_{c^{\prime}} m_{a^{\prime}} B_{c a}^{\prime}+\sum_{c} \sum_{c^{\prime}>c} m_{c^{m}} m_{c^{\prime}} \Phi_{c c^{\prime}} \\
+\sum_{a} \sum_{a^{\prime}>a} m_{a^{\prime}} m_{a^{\prime}} \Phi_{a a^{\prime}}^{\prime}
\end{gathered}
$$

The first three lines are equivalent to Pitzer's suggested single-ion activity coefficient equation. The fourth line (last two terms) is the new part. The corresponding equation for anions is analogous. The corresponding equation for the $N$-th neutral species is:

$$
\ln \gamma_{N}=2 \sum_{c} m_{c} \lambda_{N c}+2 \sum_{a} m_{a} \lambda_{N a}+\sum_{c} \sum_{a} m_{c} m_{a} \zeta_{N c a}
$$

To deal with the fact that the $\lambda_{n c}$ and $\lambda_{n a}$ are only observable in combination, Harvie, Møller, and Weare (1984) adopted the convention that:

$$
\lambda_{N, \boldsymbol{H}^{+}}=0
$$

These equations were presented for the modeling of specific systems, and are not completely general. They are missing some terms describing interactions involving neutral species. A set of complete equations is given by Clegg and Brimblecombe (1990). Their equation for the activity coefficient of a neutral solute species is:

$$
\begin{aligned}
& \ln \gamma_{N}=2 \sum_{n} m_{n} \lambda_{N n}+2 \sum_{c} m_{c} \lambda_{N c}+2 \sum_{a} m_{a} \lambda_{N a} \\
& +6 \sum_{n} \sum_{c} m_{n} m_{c} \mu_{N n c}+6 \sum_{n} \sum_{a} m_{n} m_{a} \mu_{N n a} \\
& +3 \sum_{c} m_{c}^{2} \mu_{N c c}+3 \sum_{a} m_{a}^{2} \mu_{N a a}+6 \sum_{c} \sum_{a} m_{c} m_{a} \mu_{N c a} \\
& +6 \sum_{c c^{\prime}>c} m_{c^{\prime}} m_{c^{\prime}} \mu_{N c c^{\prime}}+6 \sum_{a} \sum_{a^{\prime}>a} m_{a^{\prime} m_{a^{\prime}} \mu_{N a a^{\prime}}}
\end{aligned}
$$

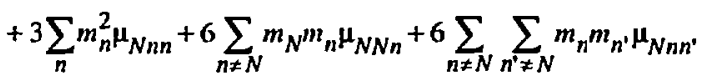

This is a complete and general representation of the activity coefficient of a neutral species in terms of all possible second order and third order primitive interaction coefficients. The first line of this equation contains the same terrs in $\lambda_{n c}$ and $\lambda_{n a}$ as appear in the Felmy-Weare equation. This line is augmented by an addition term which describes second order interactions among neutral species (and which was also pointed out by Pitzer, 1987). The third line in this equation is 
equivalent to the term in $\zeta_{N c a}$ that appears in the Felmy-Weare equation. Clegg and Brimblecombe (1990) have pointed out that this observable interaction coefficient is related to the corresponding primitive interaction coefficients by the relation:

$$
\zeta_{N M X}=6 \mu_{N M X}+\frac{3\left|z_{X}\right|}{z_{M}} \mu_{N M M}+\frac{3 z_{M}}{\left|z_{X}\right|} \mu_{N X X}
$$

The second, fourth, and fifth lines consist of terms not found in the Felmy-Weare equation.

In a solution of a pure aqueous nonelectrolyte, the activity coefficient of the neutral species takes the form:

$$
\ln \gamma_{N}=2 m_{N} \lambda_{N N}+3 m_{N}^{2} \mu_{N N N}
$$

This activity coefficient is directly observable. Hence the two interaction coefficients on the right hand side are also observable. In a study of the solubility of aqueous ammonia, Clegg and Brimblecombe (1989) found that the term including $\mu_{N N N}$ was significant only for concentrations greater than 25 molal (a solution containing more ammonia than water). They therefore dropped this term and reported model results only in terms of $\lambda_{N N}$. Similarly, Barta and Bradley (1985) found no need for a $\mu_{N N N}$ term to explain the data for pure solutions of $\mathrm{CO}_{2(a q)}, \mathrm{H}_{2} \mathrm{~S}_{(a q) \text {, and }}$ $\mathrm{CH}_{4(\mathrm{aq})}$, and no such term was apparently required by Felmy and Weare (1986) to explain the data for $B(\mathrm{OH})_{3(a q)}$. Pitzer and Silvester $(1976)$ report a significant $\mu_{N N N}$ term for undissociated phosphoric acid. This result now appears somewhat anomalous and has not been explained. The bulk of the available data, however, suggest that the $\mu_{N N N}$ term is generally insignificant in most systems of geochemical interest and can be ignored without loss of accuracy.

This result suggests that in more complex solutions, terms in $\lambda_{N N^{\prime}}, \mu_{N N N^{\prime}}, \mu_{N^{\prime} N^{\prime} N^{\prime}}$ and $\mu_{N N^{\prime} N^{n}}$ can also of ten be ignored. While there may be solutions in which the full complement of these terms are significant, one could argue that they must be so concentrated in nonelectrolyte components that they have little relevance to the study of surface waters and shallow crustal fluids (though some deep crustal fluids are rich in $\mathrm{CO}_{2}$ ). Furthermore, one could argue that to address such soJutions, it would be more appropriate to use a formalism based on a different kind of expansion than the one used in the present treatment (see Pabalan and Pitzer, 1990).

In an aqueous solution consisting of one nonelectrolyte and one electrolyte, the activity coefficient of the neutral species takes the form:

$$
\begin{gathered}
\ln \gamma_{N}=2 m_{N} \lambda_{N N}+2\left(m_{M} \lambda_{N M}+m_{X} \lambda_{N X}\right) \\
+6 m_{N}\left(m_{M} \mu_{N N M}+m_{X} \mu_{N N X}\right)+m_{M} m_{X} \zeta_{N M X}+3 m_{N}^{2} \mu_{N N N}
\end{gathered}
$$

Three new terms appear. The resemblance of the term in $\lambda_{N M}$ and $\lambda_{N X}$ to a traditional Setchenow term has been pointed out by various workers (e.g., Felmy and Weare, 1986; Pitzer, 1987). Work reported by Clegg and Brimblecombe $(1989,1990)$ for a number of such systems containing am- 
monia showed that the most important of the three new terms were the second $\left(\lambda_{N M}, \lambda_{N X}\right)$ term and the third $\left(\mu_{N N M}, \mu_{N N X}\right)$ term. They defined these using the following conventions:

$$
\begin{gathered}
\lambda_{N, C l}=0 \\
\mu_{N, N, C l}=0
\end{gathered}
$$

Note that the first of these conventions conflicts with the corresponding convention adopted by Harvie, Møller, and Weare (1984), though it matches that proposed by Pitzer and Silvester (1976) in a study of the dissociation of phosphoric acid. a weak electrolyte. Clegg and Brimblecombe found that in one system, the use of the fourth $\left(\zeta_{N M X}\right)$ term was also required, though the contribution was relatively small. No use was required of the last $\left(\mu_{N N N}\right)$ term, as was shown by fitting the data for pure aqueous ammonia.

There seems to be some disagreement in the literature regarding the above picture of the relative significance of the $\left(\mu_{N N M}, \mu_{N N X}\right)$ term versus that of the $\zeta_{N M X}$ term, although the seemingly contradictory results involve nonelectrolytes other than ammonia. We have noted above that Felmy and Weare (1986) used a $\zeta_{N M X}$ term to explain the behavior of boric acid-electrolyte mixtures. It is not clear if they considered the possibility of a $\left(\mu_{N N M}, \mu_{N N X}\right)$ term. Pitzer and Silvester (1976) found no apparent need to include a $\left(\mu_{N N M}, \mu_{N N X}\right)$ term or a $\zeta_{N M X}$ term to explain the thermodynamics of phosphoric acid dissociation in electrolyte solutions. The data on aqueous silica in electrolyte solutions of Chen and Marshall (1981), discussed by Pitzer (1987), require a $\zeta_{N M X}$ term, but no $\left(\mu_{N N M}, \mu_{N N X}\right)$ term. A similar result was obtained by Barta and Bradley (1985) for mixnures of electrolytes with $\mathrm{CO}_{2(a q)}, \mathrm{H}_{2} \mathrm{~S}_{(\mathrm{ag})}$, and $\mathrm{CH}_{4(\mathrm{aq})}$. Simonson et al. (1987) interpret data for mixtures of boric acid with sodium borate and sodium chloride and of boric acid with potassium borate and potassium chloride exclusively in terms of the first $\left(\lambda_{N N}\right)$ and second $\left(\lambda_{N M}, \lambda_{N X}\right)$ terms, using neither of the third order terms for nonelectrolyte-electrolyte interactions.

The ( $\left.\mu_{N N M}, \mu_{N N X}\right)$ term can only be observed (and hence is only significant) when the concentrations of both the nonelectrolyte and the electrolyte are sufficiently high. In contrast, evaluating the $\zeta_{N M X}$ term requires data for high concentrations of the electrolyte, but low concentrations of the nonelectrolyte will suffice. Some nonelectrolytes, such as aqueous silica, are limited to low concentrations by solubility constraints. Thus, the results of Chen and Marshall (1981) noted by Pitzer (1987) are not surprising. In the case of more soluble nonelectrolytes, the range of the available experimental data could preclude the evaluation of the $\left(\mu_{N N M}, \mu_{N N X}\right)$ term. This may be why Pitzer and Silvester (1976) reported no need for such a term to describe the data for mixtures of electrolytes with phosphoric acid and why Barta and Bradley (1985) found no need for

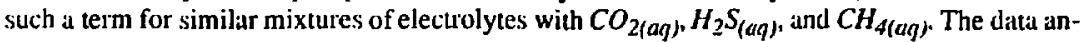
alyzed by Felmy and Weare (1986) correspond to boric acid concentrations of about one molal, which may not be high to observe this term (or reguire its use). In the case of Simonson et al. (1987), who also looked at mixtures of electrolytes and boric acid, the need for no third order terms describing nonelectrolyte-electrolyte interactions is clearly due to the fact that the concentrations of boric acid were kept low to avoid the formation of polyborate species. 
The equations for solutions containing nonelectrolytes can be considerably simplified if the model parameters are restricted to those pertaining to solutions of pure aqueous nonelectrolytes and mixtures of one nonelectrolyte and one electrolyte. This is analogous to the usual restriction in treating electrolyte solutions, in which the parameters are restricted to those pertaining to solutions of two electrolytes with a common ion. Furthermore, it seems appropriate as well to drop the terms in $\mu_{N N}$. The equation for the activity coefficient of a neutral electrolyte in electrolytenonelectrolyte mixtures then becomes:

$$
\begin{gathered}
\ln \gamma_{N}=2 m_{N} \lambda_{N N}+2\left(\sum_{c} m_{c} \lambda_{N c}+\sum_{a} m_{a} \lambda_{N a}\right) \\
+6 m_{N}\left(\sum_{c} m_{c} \mu_{N N c}+\sum_{a} m_{a} \mu_{N N a}\right)+\sum_{c} \sum_{a} m_{c} m_{a} \zeta_{N c a}
\end{gathered}
$$

The reduction in complexity is substantial. In the context of using Pitzer's equations in geochemical modeling codes, this level of complexity is probably quite adequate for dealing with nonelectrolytes in a wide range of application.

If a higher level of complexity is required, the next step is probably to add in terms in $\mu_{N N N}$ and $\lambda_{N N}$. The first of these has been discussed previously and is obtained from data on pure aqueous nonelectrolytes. The second must be obtained from mixtures of two aqueous electrolytes (one could argue that this is also analogous to the treatment of electrolytes). This higher level of complexity may suffice to deal with at least some $\mathrm{CO}_{2}$-rich deep crustal fluids and perhaps other fluids of interest in chemical engineering. However, an even higher level of complexity would probably be best addressed by a formalism based on an altermate expansion, as noted earlier.

The observability and mapping issues pertaining to the remaining parameters may be dealt with as follows. In the case of $\lambda_{N N}$, no mapping relation is required because this parameter is directly observable. The same is true of $\mu_{N N N}$ and $\lambda_{N N^{\prime}}$, if the higher level of complexity is required.

The $\lambda_{N M}$ and $\lambda_{N X}$, and $\mu_{N N M}$ and $\mu_{N N X}$, are only observable in combinations, but can be dealt with by adopting the following respective conventions:

$$
\begin{gathered}
\lambda_{N, J}=0 \\
\mu_{N, N, J}=0
\end{gathered}
$$

where $J$ is a reference ion $\left(J=H^{+}\right.$as suggested by Felmy and Weare, 1986; $J=C{ }^{\prime}$ as suggested by Pitzer and Silvester, 1976, and Clegg and Brimblecombe, 1989, 1990). In any data file used to support code calculations, the choice of reference ion must be consistent. This may require the recalculation of some published data.

The $\zeta_{N M X}$ parameter is observable and can be mapped into primitive form by adopting the following conventions:

$$
\mu_{N M M}=0
$$




$$
\begin{gathered}
\mu_{N X X}=0 \\
\mu_{N A X X}=\frac{\zeta_{N M X}}{6}
\end{gathered}
$$

These relations are analogous to those defined for the $C_{M X}^{\phi}$ parameter.

The above conventions correspond well with the current literature on the subject. However, the treatment of the $\lambda_{N M}$ and $\lambda_{N X}$, and $\mu_{N N M}$ and $\mu_{N N X}$, though valid and functional, still stands out in that it is not analogous to, or a natural extension of, the conventions which have been universally adopted in the treatment of electrolyte solutions. The logical extension, of course, is to define observable interaction coefficients to represent the primitive coefficients which can only be observed in combination, and to then follow Pitzer (1979) in determining exactly which parts of the theoretical equations constitute the non-observable part. The conventions would then be defined so as to make these parts have zero value.

The suggested process can be shown to be consistent with the above mapping conventions for all the other coefficients treated above, including $\zeta_{N M X}$. However, the process which worked so nicely for electrolytes fails to work for $\lambda_{N M^{-}}-\lambda_{N X}$, and $\mu_{N N M^{-}}-\mu_{N N X}$. We will demonstrate this for the case of the $\lambda_{N M}-\lambda_{N X}$. Application of the above equation to the case of an aqueous mixture of a neutral species $(N)$ and a neutral electrolyte $(M X)$ immediately shows that the corresponding observable combination of primitive interaction coefficients is given by:

$$
L_{N M X}=\left|z_{X}\right| \lambda_{N M}+z_{M} \lambda_{N X}
$$

In such a system, the activity coefficient of the neutral species can be written as:

$$
\ln \gamma_{N}=\frac{2}{z_{M}+\left|z_{X}\right|} v_{M X^{L}}{ }_{N M X} m_{M X}
$$

In the manner of Pitzer (1979), one can show that the relevant term in the single-ion activity coefficient for cation $M$ expands in the following manner:

$$
2 \sum_{n} m_{n} \lambda_{n M}=2 \sum_{n} m_{n} \frac{L_{n M X^{\prime}}}{\left|z_{X^{\prime}}\right|}-2 \sum_{n} m_{n} \frac{z_{M}}{\left|z_{X^{\prime}}\right|} \lambda_{n X^{\prime}}
$$

where $X^{\prime}$ is some reference anion. When $X^{\prime}$ is $C l$, we have the convention proposed by Pitzer and Silvester (1976) and followed by Clegg and Brimblecombe $(1989,1990)$. The first term on the right hand side is the relevant observable part; the second term is the non-observable part. Following the logic of Pitzer (1979), we could set the second term to zero. This would have the effect of defining the following mapping relations:

$$
\lambda_{N X^{\prime}}=0
$$




$$
\lambda_{N M}=\frac{L_{N M X}}{\left|z_{X}\right|}
$$

Although this makes the relevant non-observable part vanish in the single-ion activity coefficient equation for all cations, it forces the complementary part in the corresponding equation for anions to not vanish, as we will now show. The relevant part of the anion equation gives the following analogous result:

$$
2 \sum_{n} m_{n} \lambda_{n X}=2 \sum_{n} m_{n} \frac{L_{n M^{\prime} X}}{z_{M^{\prime}}}-2 \sum_{n} m_{n} \frac{\left|z_{X}\right|}{z_{M^{\prime}}} \lambda_{n M^{\prime}}
$$

where $M^{\prime}$ is some teference cation. As before, the second term on the right hand side is the nonobservable part. Using the above mapping equation for $\lambda_{N M}$, this can be transformed to:

$$
2 \sum_{n} m_{n} \lambda_{n X}=2 \sum_{n} m_{n} \frac{L_{n M^{\prime} X}}{z_{M^{\prime}}}-2 \sum_{n} m_{n} \frac{z_{X}}{z_{M^{\prime} X^{\prime}}} L_{n M^{\prime} X^{\prime}}
$$

Thus, under the conventions defined above, the non-observable part of the single-ion activity coefficient equation for anions does not vanish.

There are alternatives, but none are particularly outstanding. For example, one could reverse the situation and make analogous conventions so that the non-observable part of the anion equation vanishes, but then the non-observable part of the cation equation would not vanish. When $M^{n}$ is $H^{+}$, we have the convention proposed by Felmy and Weare (1986). One could also try a symmetrical mapping, based on the following relation:

$$
\lambda_{N M}=\frac{z_{M}}{\left|z_{X}\right|} \lambda_{N X}
$$

This would lead to the following mapping relations:

$$
\begin{aligned}
& \lambda_{N M}=\frac{z_{M}}{\left(z_{M}+\left|z_{X}\right|\right)} L_{N M X} \\
& \lambda_{N X}=\frac{\left|z_{X}\right|}{\left(z_{M}+\left|z_{X}\right|\right)} L_{N M X}
\end{aligned}
$$

Unfortunately, this svould lead to a non-vanishing non-observable part in the eguations for both cations and anions.

\subsubsection{Temperature and Pressure Dependence}

Pitzer's equations were originally developed and applied to conditions of $25^{\circ} \mathrm{C}$ and atmospheric pressure (e.g., Pitzer and Kim, 1974). The formalism was subsequently applied both to activity coefficients under other conditions and also to related thermodynamic properties which refiect 
the temperature and pressure dependence of the activity coefficients (see the review by Pitzer, 1987).

The first effort to extend the Pitzer formalism to high temperature was a detailed study of the properties of aqueous sodium chloride (Silvester and Pitzer, 1977). In this study, the data were fit to a complex temperature function with up to 21 parameters per observable interaction coefficient and which appears not to have been applied to any other system. In general, the early efforts concerning the temperature dependence of the activity coefficients focused mainly on estimating the first derivatives of the observable interaction coefficient parameters with respect to temperature (e.g., Silvester and Pitzer, 1978). The results of the more detailed study of sodium chloride by Silvester and Pitzer (1977; see their Figures 4, 5, and 6) suggest that these first derivatives provide an extrapolation that is reasonably accurate up to about $100^{\circ} \mathrm{C}$.

In more recent work, the temperature dependence has been expressed in various studies by a variety of different temperature functions, most of which require only 5-7 parameters per observable interaction coefficient. Pabalan and Pitzer (1987) used such equations to develop a model for the system $\mathrm{Na}-\mathrm{K}-\mathrm{Mg}-\mathrm{Cl}-\mathrm{SO}_{4}-\mathrm{OH}-\mathrm{H}_{2} \mathrm{O}$ which appears to be generally valid up to about $200^{\circ} \mathrm{C}$. Pabalan and Pitzer (1988) used equations of this type to built a model for the system $\mathrm{Na}$ $\mathrm{Cl}-\mathrm{SO}_{4}-\mathrm{OH}-\mathrm{H}_{2} \mathrm{O}$ that extends to $300^{\circ} \mathrm{C}$. Greenberg and Møller (1989), using an elaborate compound temperarure function, have constructed a model for the $\mathrm{Na}-\mathrm{K}-\mathrm{Ca}-\mathrm{Cl}-\mathrm{SO}_{4}-\mathrm{H}_{2} \mathrm{O}$ system that is valid from $0-250^{\circ} \mathrm{C}$. More recently, Spencer, Moller, and Weare (1990) have used a more compact equation to develop a model for the $\mathrm{Na}-\mathrm{K} \cdot \mathrm{Ca}-\mathrm{Mg}-\mathrm{Cl}-\mathrm{SO}_{4}-\mathrm{H}_{2} \mathrm{O}$ system at temperatures in the range -60 to $25^{\circ} \mathrm{C}$.

The pressure dependence of activity coefficients has also been looked at in the context of the Pitzer formalism. For descri tions of recent work, see Kumar (1986), Connaughton, Millero, and Pitzer (1989), and Monnin (1989).

\subsubsection{Practical Aspects}

In practice, the matter of obtaining values for the observable interaction coefficients is more complicated. Not all models based on Pitzer's equations are mutually consistent. Mixing reported data can lead to inconsistencies. For the most part, differences in reported values for the same coefficient are functions of the exact data chosen for use in the fitting process, not just whose data, but what kind or kinds of data as well. Some older reported values for the mixture parameters (e.g., Pitzer, 1979) are based on fits not employing the $E_{\theta_{M}}$, formalism, which has become firmly entrenched in more recent work.

Some differences in the values of reported Pitzer parameters are due to minor differences in the values used for the $A^{\phi}$ Debye-Hückel parameter (e.g., 0.39 versus 0.392; see Plummer et al., 1988 , p. 3, or Plummer and Parkhurst, 1990). The general problem of minor discrepancies in this and other limiting law slope parameters has been looked at in some detail by Ananthaswamy and Atkinson (1984). Recently, Archer (1990) has also looked at this problem and proposed a method for adjusting reported Pizer coefficients for minor changes in Debye-Hückel parameters without resorting to refitting the original experimental data. 
There has also been some occasional modification of the basic activity coefficient equations themselves. For example, in treating the activity coefficients of alkali sulfate salts at high temperature, Holmes and Mesmer (1986a, 1986b) changed the recommended value of the $\alpha$ parameter from 2.0 to 1.4. Also Kodytek and Dolejs (1986) have proposed a more widespread usage of the $\beta_{M X}^{(2)}$ parameter, based on the empirical grounds that better fits can be obtained for some systems. The usage of this parameter was originally restricted to the trealment of $2: 2$ electrolytes (Pitzer and Mayorga, 1973).

The formal treatment of speciation in the solutions (assumptions of which species are present) can also lead to different models. Association phenomena were first recognized in the Pitzer formalism in order to deal with phosphoric acid (Pitzer and Silvester, 1976) and sulfuric acid (Pitzer, Roy, and Silvester, 1977). In general, ion pairs have been treated formally as non-existent. An exception is in the model of Harvie, Møller, and Weare (1984), who employ three ion pair species: $\mathrm{CaCO}_{3(a q)}, \mathrm{MgCO}_{3(a q)}$, and $\mathrm{MgOH}^{+}$.

Components which form strong complexes have received relatively little attention in the Pitzer formalism, presumably because of the much greater experimental dat? requirements necessary to evaluate the greater number of parameters associated with the greater number of species. However, Millero and Byrne (1984) have used Pitzer's equations to develop a model of activity coefficients and the formation of lead chloro complexes in some concentrated electrolyte solutions. Huang (1989) has also recently looked at some examples of complex formation in the context of the Pitzer formalism. However, because strong complexing can not be represented even mathematically by the interaction coefficient formalism without taking explicit account of the associated chemical equilibria, and because such models are more difficult to develop, the practical application of the Pitzer formalism remains limited mostly to systems of relatively strong electrolytes, molecular nonelectrolytes, and a few weak nonelectrolytes.

\subsubsection{Pitzer's Equations in EQ3/6: Current Status}

The present treatment of Pitzer's equations in EQ3/6 is somewhat limited, particularly in regard to some of the advances that have been made with these equations in the past few years. These limitations have to do with the state of the existing data files which support the use of Pitzer's equations, the treatment of the temperature dependence of the interaction coefficients, and the treatment of neutral solute species.

The hmw data file is an implementation of the model of Harvie, Møller, and Weare (1984). This model is restricted to $25^{\circ} \mathrm{C}$. The pit data file is based mostly on the data summarized by Pitzer (1979). These data include the first order temperature derivatives of the interaction coefficients. The nominal temperature range of this data file is $0-100^{\circ} \mathrm{C}$. These data are not based on the currently universally accepted ${ }_{\theta} \theta$ formalism introduced by Pitzer (1975).

$\mathrm{EQ} 3 / 6$ uses or ignores the ${ }{ }_{\theta}$ formalism, depending on the value of a flag parameter on the data file. The temperature dependence, if any, is handled by using first and second order temperature derivatives of the interaction coefficients, which are expected for use at temperatures other than $25^{\circ} \mathrm{C}$. The code permits a $\beta_{M \%}^{(2)}$ parameter to be specified on the data file for any electrolyte. The 
$\alpha$ parameters are also provided on the data file for each electrolyte. Thus, non-standard values can be employed if desired.

The temperature dependence is presently limited to a representation in terms of a second-order Taylor's series in temperature. This requires the presence on the supporting data file of first and second temperature derivatives (see the EQPT User's Guide, Daveler and Wolery, 1992). No provision has yet been made for the more sophisticated representations proposed for example by $\mathrm{Pa}$ balan and Pitzer (1987) or Spencer, Møller, and Weare (1990).

EQ3/6 is presently quite limited in terms of the treatment of nonelectrolyte components by means of Pitzer's equations. This limitation is expressed in the structure of the data files and the mapping relations presently built into the EQPT data file preprocessor. These are presently set up to deal only with electrolyte parameters. However, it is possible to enter $\lambda_{N N}, \lambda_{N N}, \lambda_{N M}$, and $\lambda_{N X}$ parameters as though they were $\beta_{M X}^{(0)}$ parameters. The $\lambda_{W M}$ and $\lambda_{W X}$ parameters that are part of the model of Harvie, Møller, and Weare (1984) are included on the $h m w$ data file in this manner.

The present version of EQPT can not handle the $\zeta_{M N X}$ interaction coefficient; however.

The means of storing and representing interaction coefficient data in EQ3/6 deserves some comment. There is a natural tendency to represent $\lambda_{i j}$ by a two-dimensional array, and $\mu_{i j k}$ by a threedimensional array. However, arrays of this type would be sparse (for example, $\lambda_{i j}=0$ for many $i, j)$. and many of the entries would be duplicates of others $\left(\lambda_{i j}=\lambda_{j i}\right.$, etc.). Therefore, the $\lambda_{i j}$ are represented instead by three parallel one-dimensional arrays. The first contains the $\lambda_{i j}$ values themselves, the second contains indices identifying the $i$-th species, and the third identifies the $j$ th species. The treatment is analogous for $\mu_{i j k}$, which only requires an additional array to identify the $k$-th species. These arrays are constructed from data listed on the data0 data files. Coefficients which must be zero by virtue of the mapping relations or other conventions are not included in the constructed arrays. Also, the storage scheme treats for example $\lambda_{i j}$ and $\lambda_{j i}$ as one coefficient, not two. 


\section{Activity Coefficients of Solid Solution Components}

\subsection{Introduction}

The thermodynamic activities $\left(a_{i}\right)$ of solid solution components are always defined on the basis of mole fractions. Thus, they can be described by the product of their mole fractions $\left(x_{i}\right)$ and their rational (mole fraction) activity coefficients $\left(\lambda_{i}\right)$ :

$$
a_{i}=x_{i} \lambda_{i}
$$

The same treatment is typically applied to all components in non-aqueous liquid phases. It is also applied to water in aqueous solutions (cf. Chapter 3).

Mole fraction ideality is the reference ideality when dealing with solid solutions. Therefore, the corresponding excess Gibbs energy is $G^{E X x}$ (see Chapter 3). The relevant differential equation linking this with the mole fraction activity coefficients is:

$$
\ln \lambda_{i}=\frac{1}{R T} \frac{\partial G^{E X x}}{\partial n_{i}}
$$

where $R$ is the gas constant and $T$ the absolute temperature. Given an expression for the excess Gibbs energy, this equation gives a guaranteed route to thermodynamically consistert results (cf. Wolery, 1990).

Problems involving the thermodynamic consistency of activity coefficients in non-aqueous phases seem to be uncommon. However, consistency may be tested using various relations, such as the following form of the cross-differentiation rule (cf. Wolrry, 1990):

$$
\frac{\partial \ln \lambda_{j}}{\partial n_{i}}=\frac{\partial \ln \lambda_{i}}{\partial n_{j}}
$$

The issue of sufficiency in proving consistency using this and related equations (Gibbs-Duhem equations and sum rules) is addressed by Wolery (1990).

In most speciation-solubiiity calculations, the activity coefficients of so id solution components only affect the corresponding calculated saturation indices; they do no: change the model of the aqueous solution itself (i.e., the speciation). However, if an equilibrium relation involving a solid solution phase is used as a constraint in defining a speciation-solubility problem, all of the model results may be affected by the choice of activity coefficient model. The results may similarly affected when such a consuraint is used in mass transfer calculations. including reaction path calculations.

Mixing tends to stabilize a solid solution relative to its end-merber components. Thus, an aqueous solution may be supersaturated with respect to a solid solution, yet undersaturated with respect to each of the pure end members. Consequently, a solid solution may form in a system in which some or none of the pure end members would form. This effect is true in the ideal case, in 
which the activity coefficient has a value of unity. If the activity coefficient is less than unity, this stabilizing effect is increased. If it is greater than unity, it is lessened.

In general, the activity coefficient of a solid solution component depends on the composition of the solid solution. This is in turn is normally expressed in terms of the mole fractions of the components. In order to use an equilibrium constraint involving a solid solution component in a speciation-solubility calcuiation, the user must provide this composition in order to allow calculation of the activity coefficient of the component involved. In mass transfer calculations involving solid solutions in equilibrium with an aqueous solution, the solid solution composition is itself a subset of the unknowns to be calculated. In speciation-solubility and other kinds of equilibrium calculations, it is necessary to calculate a saturation index for a solid solution which is not presumed to be in equilibrium with the aqueous phase. This presents a problem, because no composition is defined. This is solved in EQ3/6 by finding the composition which maximizes the computed saturation index (Bourcier, 1985, 1989).

In liquid solutions, the solutes may mix over the whole volume of the solution. This type of mixing is sometimes referred to as molecular mixing. It is commonly applied to non-aqueous liquids, such as a solution composed of hydrocarbons. In the ideal case, the activity coefficient of each component is unity. In aquesus solutions and other solutions involving a solvent with a high dielectric constant, this concept is modified to account for ionic dissociation. The concept of molecular mixing has been applied many times to solid solutions (cf. the examples presented later in this chapter), and is predicated on the use of end-member components (for example, calcite $\left[\mathrm{CaCO} \mathrm{C}_{3}\right]$ and magnesite $\left[\mathrm{MgCO}_{3}\right]$ in magnesian calcite $\left.\left[(\mathrm{Ca}, \mathrm{Mg}) \mathrm{CO}_{3}\right]\right)$. The activity coefficients of these components in the non-ideal case are then described using interaction coefficients more or less resembling those used in Pitzer's equations to describe the activity coefficients of aqueous species.

In crystalline solids, mixing tends rather strongly to occur over well-defined sites in the crystal structure (see for example Wood and Fraser, 1977, or Nordstrom and Munoz, 1985). Some ions may mix over more than one kind of site. Vacancies may be involved in the mixing process. They may be created or destroyed by substitutions of one ion for ' ' niher of different elecs ical charge. Mixing which takes account of such effects is referred to as stte mixing. In site-mixing models, the concept of ideality is modified from that appropriate to molecular mixing, though still based on the mole fractions of components. It is possible to utilize as the components species such as ions, vacancies, and framework moieties instead of end members. However, the more common practice is to continue using end-member companents. This is followed in the present version of EQ3/6. However, the activity coefficient of an end-member component in an ideal site mixing model may have a value other than unity. A site-mixing model will appear to be ideal in this sense only if there is only one site, an ion substitutes for others of the same charge type, and vacancies are not present on the site. Site mixing then effectively reduces to molecular mixing.

Nearly all of the site-mixing models that have been proposed for the various solid solutions are ideal in the site-mixing sense (see for example Viani and Bruton, 1992). The only paraneters of such models are site-mixing parameters. It is possible to consider site-mixing modeis which are non-ideal sven in the site-mixing sense. These would be described by both site-mixing parameters and interaction coefficients. No models of this type are presently treated in EQ3/6. 
In EQ3/6, all solid solution models are defined on the supporting data fle (see Chapters 3 and 4 of the EQPT User's Guide, Daveler and Wolery, 1992). At present (through the R16 set of data files), only the com file contains any solid solutions. All of these are treated with ideal site-mixing models (the exception being olivine, which is treated according to a binary regular solution model). The actual types of models used on the data fils are defined by the jsol flag array. The corresponding parameters (site-mixing parameters, interaction coefficients, and parameters used to compute interaction coefficients) are stored on the data file in the spx array. The elements of

this array are represented below as $p_{k \psi}$ In EQ3NR and EQ6, solid solutions are presently ignored unless the option switch iopt4 is set to a value greater than or equal to 1 .

The various models presently treated in EQ3/6 are discussed in the following sections. To avoid confusion, we will write the activity, mole fraction, activity coefficient, and related parameters of a solid solution component with "a " $\sigma \psi$ " subscript in place of " $i$." Here $\sigma$ denotes the component itself ( $($ akes the place of " $i$ "), and $\psi$ the solid solution (in onder to be completely explicit about which solid solution is being addressed).

\subsection{Ideal Solution, with One Optional Site-Mixing Farameter}

The first activity coefficient model for solid solutions in EQ3/6 is for an ideal solution in either the molecular-mixing sense or a limited site-mixing model in which mixing is confined to one site and vacancies are ignored. The former is a special case of the latter. This model corresponds to jsol = 1 and is characterized by the equation (Wood and Fraser, 1977; Viani and Bruton, 1992):

$$
a_{\sigma \psi}=x_{\sigma \psi}^{N_{\psi}}
$$

where $N_{\psi}$ is the site mixing parameter. 1 nis formulation is equivalent to:

$$
\log \lambda_{\sigma \psi}=\left(N_{\Psi}-1\right) \log x_{\sigma \psi}
$$

If $N_{\psi}=1$, the above model is mathematically equivalent to an ideal molecular-mixing model $\left(\log \lambda_{\mathrm{\alpha \psi}}=0\right)$.

The $N_{\psi}$ parameter is stoichiometric in nature. In essence, it is the number of formula units of the site on which mixing occurs per formu'a unit of the solid solution framework. In principle, the formula for all the end-member components of a solid solution can be written so as to yield $N_{\psi}$ $=1$, hence $\log \lambda_{\sigma \psi}=0$.

In the case of heterovalent single-site solid solutions such as clays and zeolites, vacancies are involved. In order to simplify the s.? lution model and preserve the simple relationship defined by eqs (184) and (185), Viini and Bruton (1992) have chosen to treat such solid solutions according to a model in which the mixing entities are ions or ion-vacancy complexes. Thus, two sodium ion entities might mix .. ith a calcium ion-vacancy entity.

The $N_{\psi}$ parameter is oblained from the parameters read from the data file according to: 


$$
N_{\psi}=p_{7 \Psi}
$$

\subsection{Third-Order Maclaurin Model for a Binary Solution}

The third-order Maclaurin model for a binary solution corresponds to jsol = 2. It is taken from Helgeson $i$ al. (1970). The activity coefficients of the two end-member components are given by:

$$
\begin{gathered}
\log \lambda_{1 \psi}=\frac{1}{2.303 R T}\left[-\left(\frac{W_{2 \psi}}{2}\right) x_{2}^{2}-\left(\frac{W_{3 \psi}}{3}\right) x_{2}^{3}\right] \\
\log \lambda_{2 \psi}=\frac{1}{2.303 R T}\left[-\left(\frac{W_{2 \psi}+W_{3 \psi}}{2}\right) x_{1}^{2}+\left(\frac{W_{3 \psi}}{3}\right) x_{1}^{3}+\left(W_{1 \psi}+\frac{W_{2 \psi}}{2}+\frac{W_{3 \psi}}{6}\right)\right]
\end{gathered}
$$

Here $W_{I \psi}, W_{2 \psi r}$ and $W_{3 \psi}$ are interaction coefficients. There are no site-mixing parameters.

The formulation represented by eqs (187) and (188) is highly unsymmetrical. In order to satisfy the condition that $\log \lambda_{2 \psi} \rightarrow 0$ as $x_{1} \rightarrow 0$, the interaction coefficients are required to satisfy the relation:

$$
W_{1 \Psi}=-\frac{W_{2 \Psi}}{2}-\frac{W_{3 \Psi}}{6}
$$

The interaction coefficients are obtained from the parameters read from the data file according to:

$$
\begin{aligned}
& w_{1 \psi}=p_{1 \psi} \\
& w_{2 \psi}=p_{2 \psi} \\
& w_{3 \psi}=p_{3 \psi}
\end{aligned}
$$

However, $W_{I \Psi}$ is actually recalculated using eq (189).

\subsection{Regular Solution Model for a Binary Solution}

The regular solution model for a binary solution corresponds to $\mathbf{j s o l}=3$. It is also called a parabolic Maclaurin model. For a discussion of this model, see Saxena (1973, p. 11-12). The activity coefficients of the two end-member components are given by:

$$
\begin{aligned}
& \log \lambda_{1 \Psi}=\frac{1}{2.303 R T} W_{\psi} x_{2}^{2} \\
& \log \lambda_{2 \Psi}=\frac{1}{2.303 R T} W_{\psi} x_{1}^{2}
\end{aligned}
$$


Here $W_{\psi}$ is the single interaction coefficient. There are no site-mixing parameters. This formulation is symmetrical.

The interaction coefficients are obtained from the parameters read from the data file according to:

$$
W_{\psi}=p_{1 \psi}+p_{2 \Psi} T+p_{3 \psi} P
$$

Thus, the interaction coefficient in this model can be treated as a function of temperature and pressure. On the com data file in the R10 and R16 sets, there is a regular solution model for the solid solution olivine. The $p_{2 \psi}$ and $p_{3 \psi}$ parameters are set to zero, so the interaction coefficient is actually treated as a constant. A non-unit site-mixing parameter is also given in the $p_{7 \psi}$ parameter, but this is not used.

\subsection{Cubic Maclaurin Model for a Binary Solution}

The cubic Maclaurin muciel for a binary solution corresponds to jsol $=4$. For a discussion of this model, see Saxena (1973, p. 16). The activity coefficients of the two end-member components are given by:

$$
\begin{aligned}
& \log \lambda_{1 \Psi}=\frac{1}{2.303 R T}\left[\left(2 W_{2 \Psi}-W_{1 \Psi}\right) x_{2}^{2}+2\left(W_{1 \Psi}-W_{2 \Psi}\right) x_{2}^{3}\right] \\
& \log \lambda_{2 \Psi}=\frac{1}{2.303 R T}\left[\left(2 W_{1 \Psi}-W_{2 \Psi}\right) x_{1}^{2}+2\left(W_{2 \Psi}-W_{1 \psi}\right) x_{1}^{3}\right]
\end{aligned}
$$

Here $W_{I \psi r}$ and $W_{2 \psi}$ are interaction coefficients. There are no site-mixing parameters. This formulation is asymmetrical.

The interaction coefficients are obtained from the parameters read from the data file according to:

$$
\begin{aligned}
& W_{1 \Psi}=p_{1 \Psi}+p_{2 \psi} T+p_{3 \Psi} P \\
& w_{2 \Psi}=p_{4 \Psi}+p_{5 \psi} T+p_{6 \psi} P
\end{aligned}
$$

\subsection{Guggenheim Polynomial Model for a Binary Solution}

The Guggenheim polynomial model for a binary solution corresponds to jsol $=5$. For a discussion of this model, see Saxena (1973, p. 14-15). The activity coefficients of the two end-member components are given by:

$$
\begin{aligned}
& \log \lambda_{1 \psi}=\frac{1}{2.303 R T}\left[\left(W_{1 \Psi}+3 W_{2 \Psi}+5 W_{3 \Psi}\right) x_{2}^{2}+\left(-4 W_{2 \Psi}-16 W_{3 \Psi}\right) x_{2}^{3}+12 W_{3 \Psi} x_{2}^{4}\right] \\
& \log \lambda_{2 \Psi}=\frac{1}{2.303 R T}\left[\left(W_{1 \Psi}-3 W_{2 \Psi}+5 W_{3 \Psi}\right) x_{1}^{2}+\left(4 W_{2 \Psi}-16 W_{3 \Psi}\right) x_{1}^{3}+12 W_{3 \Psi} x_{1}^{4}\right]
\end{aligned}
$$


Here $W_{I \psi} W_{2 \psi n}$ and $W_{3 \psi}$ are interaction coefficients. There are no site-mixing parameters. This formulation is asymmetrical.

The interaction coefficients are obtained from the parameters read from the data file according to:

$$
\begin{aligned}
& w_{1 \psi}=p_{1 \psi}+p_{2 \psi} T+p_{3 \psi} T^{2} \\
& w_{2 \psi}=p_{4 \psi}+p_{5 \psi} T+p_{6 \psi} T^{2} \\
& W_{3 \psi}=p_{7 \psi}+p_{8 \psi} T+p_{9 \psi} T^{2}
\end{aligned}
$$

The full form of this model can be used in the present version of $\mathrm{EQ} 3 / 6$, although the parameters $p_{k \psi}$ for $k \geq 7$ are now intended to be reserved for site-mixing parameters.

\subsection{Regular Solution Model for a Temary Solution}

The regular solution model for a ternary solution corresponds to jsol $=6$. For a discussion of this model, see Prigogine and Defay (1954, p. 257). The activity coefficients of the three end-member components are given by:

$$
\begin{aligned}
& \log \lambda_{1 \psi}=\frac{1}{2.303 R T}\left[W_{12 \psi} x_{2}^{2}+W_{13 \psi} x_{3}^{2}+\left(W_{12 \psi}-W_{23 \psi}+W_{13 \psi}\right) x_{2} x_{3}\right] \\
& \log \lambda_{2 \psi}=\frac{1}{2.303 R T}\left[W_{12 \psi} x_{1}^{2}+W_{23 \psi} x_{3}^{2}+\left(W_{12 \psi}-W_{13 \psi}+W_{23 \psi}\right) x_{1} x_{3}\right] \\
& \log \lambda_{3 \psi}=\frac{1}{2.303 R T}\left[W_{13 \psi} x_{1}^{2}+W_{23 \psi} x_{2}^{2}+\left(W_{13 \psi}-W_{12 \psi}+W_{23 \psi}\right) x_{1} x_{2}\right]
\end{aligned}
$$

Here $W_{1 \psi} W_{2 \psi}$ and $W_{3 \psi}$ are interaction coefficients. There are no site-mixing parameters. This formulation is symmetrical.

The interaction coefficjents are obtained from the parameters read from the data file according to:

$$
\begin{aligned}
& W_{12 \psi}=p_{1 \psi} \\
& W_{13 \psi}=p_{2 \psi} \\
& W_{23 \psi}=p_{3 \psi}
\end{aligned}
$$




\section{Basis Species: Key Concepts}

\subsection{Basis Species}

In the EQ3/6 system, there is a set of master or basis species. In Chapter 2, this concept was introduced via the notion that one such species is associated with each chemical element and its associated mass balance (e.g., $\mathrm{Na}^{+}$for $\mathrm{Na}$ ). If oxidation-reduction is considered, one additional species such as $O_{2\{g\}}$ for $e^{-}$must be added, which is associated with charge balance. Every remaining species (aqueous, mineral, or gas) is formally associated with a reaction which destroys it. For example, an agueous complex is paired with its dissociation reaction, and a mineral with its dissolution reaction. The basis species are used as a set of generalized "building blocks" in writing chemical reactions. The reactions are then written in terms of only the single associated species and the set of basis aqueous species.

We will call a basis set as defined above a strict basis set. It is the minimal basis set required for chemical modeling. The number of species in this set, in the general case including a redox species, is given by:

$$
s_{B}=\varepsilon_{T}+1
$$

where $\varepsilon_{T}$ is the number of chemical clements in the system of interest.The redox species itself will be denoted as the $s_{B}$-th species. In the case of systems in which there is no oxidation-reduction, we will simply treat the redox species as being inactive.

A speciation-solubility problem concerning an aqueous solution deals only with mass balances involving species in one (aqueous) phase. Therefore, the basis set in EQ3NR consists entirely of aqueous species. These are defined (at least initially) on the supporting data file. One is $\mathrm{H}_{2} \mathrm{O}_{(i)}$, the solvent. The redox species used in $\mathrm{EQ} 3 / 6$ is $O_{2(g)}$, which is treated in this context as a fictive aqueous species; the conventional $e^{-}$used by some other modeling codes is another example of such. The other basis species are simple species likely to dominate their respective mass balance relationships, at least in many instances.

Basis species are usually chosen as mono-elemental species such as $\mathrm{Na}^{+}$and $\mathrm{Ca}^{2+}$. Some are also comprised of oxygen and/or hydrogen (e.g., $\mathrm{SO}_{4}{ }^{2-}$ and $\mathrm{B}(\mathrm{OH})_{3(\mathrm{aq})}$ ). No basis species on a supporting data file is permitted to be comprised of more than one chemical element other than oxygen or hydrogen. The purpose of this restriction is to avoid certain problems that would otherwise arise in defining the total concentrations of the basis species. Such problems do not arise in the case of dealing with elemental oxygen and hydrogen because no meaningful analytical values exist for the total concentrations of the associated basis species, $\mathrm{H}_{2} \mathrm{O}_{(1)}$ and $\mathrm{H}^{+}$or of these elements themselves. The concenuration of water as measured by its mole fraction is implicilly fixed by the concentrations of the solute components. The concentration of the hydrogen ion is analytically determined via the $p H$ or some other approatch not involving a total concentration.

Using a strict basis set, all mass balance relationships can be defined in terms of chemical elements and the coefficients describing the elemental compositions of all species. The charge bal- 
ance relationship can be defined in terms of the electrical charges of the species. All non-basis species appearing in these balance equations are related to the basis species via the associated chemical reactions. The concentrations of these non-basis species are then determined by the concentrations of the basis species through the associated mass action equations, assuming that the activity coefficients appearing in these equations are known. Thus, if the concentrations of the basis species are known, they may be used to span (compute) the complete speciation of the system. In mathematics, a set with such properties is usually called a basis, which is actually where the term basis set in the present context is derived.

In thermodynamic modeling, one deals in an algebraic sense with $n$ equations in $n$ unknowns. The use of a basis set which is strict requires assuming that the concentration of every non-basis species appearing in a balance equation satisfies a corresponding mass action equation. This has the effect of requiring the modeled system to be in a complete state of internal chemical equilibrium. There is simply no mechanism in this construction to deal with even one simple reaction in a state of disequilibrium. The concept of internal equilibrium as used here refers to a system which excludes any non-basis species that do not appear in the balance equations. Thus, an aqueous solution may be in a state of intemal equilibrium, but still supersaturated with respect to calcite. The mineral in this context is a non-basis species, but it does not appear in the balance equations which describe the aqueous solution. The system consisting of the same aqueous solution plus the mineral, however, is not in a state of internal equilibrium.

In EQ3NR, the modeled system consists exclusively of the aqueous solution. Systems including other phases are treated in EQ6. Nevertheless, it is apparent from the above example that in order to model systems with some intemal disequilibrium, one must expand the basis set beyond the confines of the strict basis. In the example given above, we would make the mineral a basis species. However, since the associated reaction is presumed to be in disequilibrium, the associated mass action equation is not used as a governing constraint. In order to maintain a balance of $n$ equations in $n$ unknowns, it is necessary to introduce a mass new balance equation for the new basis species. In the present example, this is just a statement of how much of the mineral is present in the system. Note that this is a new kind of mass balance relation not related to a chemical element.

The same principle holds in modeling an aqueous solution. One might wish to compute a model in which $\mathrm{Fe}^{3+}$ is not in equilibrium with $\mathrm{Fe}^{2+}$. If $\mathrm{Fe}^{2+}$ is already in the strict basis set, one must add $\mathrm{Fe}^{3+}$ to the basis set (or vice versa). In this case, the situation is more complicated, as the new basis species may have its own ion pairs and complexes appearing in the associated mass balance. This is a simple concept. However, it requires rethinking the description of mass balances, as the number of mass balance equations now exceeds the number of chemical elements. As we will show, a better concept is to associate the mass balance relations with corresponding basis species, not with the chemical elements.

We now show how to develop this more generalized concept for defining mass balance relations. Consider the following reaction:

$$
\mathrm{HgCl}_{3}=\mathrm{Hg}^{2+}+3 \mathrm{Cl}^{-}
$$


This is represented in the software by paired arrays of reaction coefficients (floating point numbers) and names of the corresponding species (character variables). If you ask the question, how many chlorides is the non-basis species on the left hand side equivalent to, a human being will invariably answer the question by looking at the subscript " 3 " in the chemical formula of the species. In the software, this is equivalent to looking up the elemental composition of the species in the appropriate data array. Thus, this mechanism produces the required coefficient for evaluating the contribution of this species to a mass balance relation based on a chemical element. This is really the answer to the question, to how many chlorines (not chlorides) is this species equivalent. This is not what is presently desired, and a different approach is required.

The original question can be more accurately answered by looking at the reaction coefficients. Since reactions must satisfy mass and charge balance to be valid, the required information must be available there. A human being would probably answer the question by looking at the coefficient on the right hand side of the reaction. However, the coefficient of the non-basis species on the left hand side must also be considered to obtain the correct answer in the general case. To emphasize this, we note that the reaction can also be written as:

$$
2 \mathrm{HgCl}_{3}=2 \mathrm{Hg}^{2+}+6 \mathrm{Cl}
$$

Although a reaction whose coefficients have not becil reduced to the lowest common denominator is unlikely to be written in any of the EQ3/6 data files, it is not prohibited. Also, some reactions written with a unit coefficient for the associated non-basis species require fractional coefficients. An example is:

$$
\mathrm{Fe}^{3+}+\frac{1}{2} \mathrm{H}_{2} \mathrm{O}_{(!)}=\mathrm{Fe}^{2+}+\mathrm{H}^{+}+\frac{1}{4} \mathrm{O}_{2(g)}
$$

One might reasonably wish to avoid the fractions and write instead:

$$
4 \mathrm{Fe}^{3+}+2 \mathrm{H}_{2} \mathrm{O}_{(l)}=4 \mathrm{Fe}^{2+}+4 \mathrm{H}^{+}+\mathrm{O}_{2(\mathrm{~g})}
$$

Furthermore, certain actions taken by the code as it executes, such as basis switching, may cause a reaction to be rewritten, and there is no general restriction requiring the new reaction to have a unit coefficient for the associated non-basis species.

In the software, the coefficients of products are defined as positive numbers and those of reactants as negative ones. For the basis species, these coefficients are symbolized by $b_{s^{\prime} r}$ where $s^{\prime}$ denotes a basis species and $r$ the reaction. The non-basis species associated with the $r$-th aqueous reaction is denoted by $s^{\prime \prime}$, and its reaction coefficient is symbolized by $b_{s " r}$. Thus, the factor giving the stoichiometric equivilence of such a species to the $s^{\prime}$-th basis species is given by:

$$
u_{s^{\prime \prime} s^{*}}=-\frac{b_{s^{*} r}}{b_{s " r}}
$$

In a speciation-solubility problem, the mass, balance equation for the $s^{\prime}$-th basis species is the: 


$$
m_{T, s^{\prime}}=m_{s^{\prime}}+\sum_{r=1}^{r_{T}} u_{s^{\prime \prime}} s^{\prime} m_{s^{\prime \prime}}
$$

where $r$ is the reaction associated with the $s^{\prime \prime}-$ th species $\left(r=s^{\prime \prime}-s_{B}\right.$; see Section 9.2) and $r_{T}$ is the number of reactions for the dissociation of non-basis aqueous species. Considerable care must be used in the application of such a formulation. Mathematically, it is quite rigorous. Physically, however, there are some potential problems. The quantity on the left hand side may or may not correspond to something that can be obtained by chemical analysis and therefore have physical as well as mathematical meaning. The formulation can be applied to any basis species. In the case of $O_{2\left(z^{\prime}\right)}$ or $e^{-}$, thesc have no physical meaning, as these are only hypothetical agueous species in the first place. In the case of $\mathrm{H}^{+}$, the total concentration has no physical significance. Its value is uniquely established only because one normally chooses to put this species in the basis set instead of $\mathrm{OH}^{\circ}$. In the case of $\mathrm{H}_{2} \mathrm{O}_{(j)}$, the computed cotal concentration is also technically nonphysical and depends on which of $\mathrm{H}^{+}$or $\mathrm{OH}^{-}$is chosen as a basis species.

It was pointed uut earlier that basis species on the EQ3/6 data files are restricted in composition to no more than one chemical element other that oxygen and hydrogen. This is done to protect the physical meaning of the total concentrations of basis species other than $\mathrm{H}_{2} \mathrm{O}_{(l)}, \mathrm{H}^{+}$, and $\mathrm{O}_{2(g)}$, for which there is no possibility of physical meanir.g, anyway. To illustrate the problem, consider the following three reactions in which $\mathrm{HgCl}_{3}{ }^{-}$is used as a basis species in place of $\mathrm{Hg}^{2+}$ :

$$
\begin{gathered}
\mathrm{Hg}^{2+}+3 \mathrm{Cl}^{-}=\mathrm{HgCl}_{3} \\
\mathrm{HgBr}_{3}^{-}+3 \mathrm{Cl}=\mathrm{HgCl}_{3}+3 \mathrm{Br}^{-}
\end{gathered}
$$

Consider the mass balance of chloride and the contribution to it from $\mathrm{Hg}^{2+}$. Applying eq (216) to the first reaction above gives a stoichiometric coefficic. $t$ of -3 . The same result is obtained for the bromide complex in the second reaction. The chloride complex itself has a stoichiometric coefficient of zero.

In EQ3NR and EQ6, the chloride complex in the above example is likely to strongly dominate the mass balance of dissolved mercury, giving an incentive to consider switching it into :he basis in place of the mercuric ion. The codes deal with this situation by continuing to define the stoichiometric factors appearing in the mass balance relations in terms the reactions as they were written prior to basis switching, modified only for stoichiometric factors relating the new basis species to the old ones.

\subsection{Organization and Treatment of Basis Species}

The set of basis species on an EQ3/6 datti file is divided into two parts: the strict basis and the anxiliary basis. The species in the strict basis set correspond one-to-one with the chemical elements, except for $O_{2(g)}$, which is used as a hypothetical aqueous species, and which corresponds to charge balance. These species appear first in the overall list of aqueous species. The solvent, 
$\mathrm{H}_{2} \mathrm{O}_{(l)}$, is the first aqueous species. The hypothetical aqueous species $\mathrm{O}_{2(g)}$ is the last. The species in the strict basis set are not associated with any reactions, as are all other species.

The auxiliary basis species follow the strict basis species. For the most part, they represent chemical elements in different oxidation states. However, they may also include any species which do not readily equilibrate with other basis species according to the associated reactions. Auxiliary basis species are used like strict basis species as "building blocks" in writing reactions for various species on the data file. In EQ3NR, an auxiliary basis species may be treated as either a basis species or a non-basis species. The choice is up to the user in each case. By default, an auxiliary basis species is eliminated from the active basis set (except for $O_{2(a q)}$ and $H_{2(a q)}$, which are special cases). Any reactions for other species written in terms of this species will be rewritten to reflect this. However, an auxiliary basis species is treated as an active basis species if the user provides an appropriate matching input on the input file, such as a total concentration. The non-basis aqueous species follow the auxiliary basis species. In the present version of $\mathrm{EQ} 3 / 6$, a species defined as a non-basis species on the data file can not be treated as an active basis species unless it is switched with an existing member of this set. This prevents defining an additional mass balance relation for this species. If it is desired to use such a species in the active basis set for the purpose of defining an additional such relation, it is necessary to modify the data file, moving the species into the auxiliary basis set.

An input model constraint, such as a total concentration, is required for each master aqueous species in order to perform a speciation-solubility calculation. However, as discussed in Chapter 6, there are limitations on the constraints that can be placed on a given basis species, depending on whether it is in the strict basis or the auxiliary basis. The user needs to be keenly aware of which species are in which set. Users should consult either the relevant data0 data file or the slist (species list) file written by EQPT when it processes this file.

The user may specify selected examples of basis-switching on the input file. This provides a means of changing the set of basis species at run time. For example, a strict basis species may be exchanged with an auxiliary basis species. This puts the latter in the strict basis, the former in the auxiliary set. A basis species may also be switched with a non-basis species. A basis switch causes reactions to be re-written in terms of the new basis set. Judicious basis switching can improve the code numerics, sometimes making the difference in whether the iteration converges or not. As a general rule, a basis species should not make up an insignificant part of the associated mass balance when a total concentration is used as the input constraint. The compositional restrictions on basis species on the EQ3/6 data file do not apply to basis switching made when running EQ3NR or EQ6.

A basis switch involving a non-basis species causes the corresponding input constraints (such as those represented by the csp and uphase parameters; see Chapter 6 ), to be reassigned to the species brought into the basis set. For exampic, if $\mathrm{A} /(\mathrm{OH})_{4}{ }^{+}$is to be switched into the basis set in place of $A l^{3+}$, for which jflag = $16(\log$ activity constant) and $\operatorname{csp}=-5.0$ (the desired value), the model actually specified is one in which the log activity of $A(l O H)_{4}-$ is -5.0 . In this case, specifying a basis switch actually changes the definition of the problem. However, in a switch involving a basis species constrained to satisfy a specified total concentration, the total concentration is 
recomputed by a stoichiometric adjustment to match the species moved into the basis. In this case, the problem itself is not really changed; it is merely expressed in different terms.

Setting the jflag $=\mathbf{3 0}$ for an auxiliary basis species causes the species to be eliminated from the active basis set. As noted above, this is the default condition for most such species. Elimination from the active basis set causes reactions originally written in terms of the eliminated species to be rewritten. For example, consider the following reaction:

$$
\mathrm{FeSO}_{4}^{+}=\mathrm{Fe}^{3+}+\mathrm{SO}_{4}^{2-}
$$

Elimination of $\mathrm{Fe}^{3+}$ from the basis set causes this to be rewritten as:

$$
\mathrm{FeSO}_{4}^{+}+\frac{1}{2} \mathrm{H}_{2} \mathrm{O}_{(\mathrm{l})}=\mathrm{Fe}^{2+}+\mathrm{H}^{+}+\frac{1}{4} \mathrm{O}_{2(g)}+\mathrm{SO}_{4}^{2-}
$$

Thus, $\mathrm{FeSO}_{4}{ }^{+}$now appears to be, and is treated as, a complex of $\mathrm{Fe}^{2+}$. Elimination thus has the effect of combining mass balances. In this case, $\mathrm{Fe}^{3+}$ and its complexes are folded into the mass balance for $\mathrm{Fe}^{2+}$. Note that the reaction for $\mathrm{FeSO}_{4}{ }^{+}$on the data file must be the first, not the second, of the two above reactions. Otherwise, $\mathrm{FeSO}_{4}{ }^{+}$would have been considered a complex of $\mathrm{Fe}^{2+}$ from the start. If one did not eliminate $\mathrm{Fe}^{3+}$ from the active basis set, $\mathrm{FeSO}_{4}{ }^{+}$would be incorrectly folded in to the mass balance for $\mathrm{Fe}^{2+}$, and missing from that of $\mathrm{Fe}^{3+}$.

In the majority of cases, there are only one or two auxiliary basis species for a given chemical element. When there is more than one such auxiliary basis species, the species can be "chained." The first such species must be related through its associated reaction to the corresponding strict basis species. This can also be done in the case of the second, third, etc., such basis species. However, the second such auxiliary basis species could be durectly related to the first such auxiliary basis species. The third could be directly related to the first or second, etc. Of course, a non-basis species can be directly related to any basis species.

Chaining is not significant in the case of most chemical elements. Carbon, however, is an exception. A large number of organic species are now present on the com and sup cata files because of the inclusion of such in SUPCRT92 from the work of Schock and Helgeson (1990). Several of these are treated as auxiliary basis species, the majority as non-basis species of which all are directly related to one of the organic species in the auxiliary basis. The basic problem with organics in a geochemical modeling code is that they may often be treated as complexes of $\mathrm{HCO}_{3}{ }^{*}$ when this is not what is desired. In the "R7" versions of the com and sup data files, each of the several organic species in the auxiliary basis set is directly related to $\mathrm{HCO}_{3}^{-}$. In order to model organic-free systems without the unexpected appearance of organics in the model, it is necessary to enter on the input file a zero concentration for each organic speries in the auxiliary basis set. In future versions of the data files, one such species (probably 'acetic acid(aq)') may be set up as a sont of master organic species. Only this organic species will be directly related to $\mathrm{HCO}_{3}^{-}$. All other organic species in the auxiliary basis set will be directly related to this master organic spe- 
cies. Then all organics can be kept out of a computed model by entering on the input file a zero concentration for just the master organic species.

\subsection{Stoichiometric Conversions of Analytical Data}

The analytical data used to define speciation-solubility problems in EQ3NR pertain to the basis species on the supporting data file employed in a given nun. To use the code correctly, one must know what the species are, and it is often necessary to correct the analytical data one is provided in order to provide a stoichiometric match.

For example, the river wa ar test case of Nordstrom et al. (1979) includes the following data:

- Si $8.52 \mathrm{mg} / \mathrm{L}$.

- B $0.050 \mathrm{mg} / \mathrm{L}$.

- $\mathrm{PO}_{4}{ }^{3-} 0.210 \mathrm{mg} / \mathrm{L}$.

The species on the data0.com data file which respectively correspond to these components are $\mathrm{SiO}_{2(\mathrm{aq})}, \mathrm{B}(\mathrm{OH})_{3(a q)}$, and $\mathrm{HPO}_{4}{ }^{2-}$. The problem is that, for example, $8.52 \mathrm{mg} / \mathrm{L}$ of $\mathrm{Si}$ is not equivalent to $8.52 \mathrm{mg} / \mathrm{L}$ of $\mathrm{SiO}_{2(\text { oq) }}$. A stoichiometric conversion, of the sort common in analytical chemistry, must be done. This is illustrated in the present case by:

$$
C_{m g / L, S i O_{2(a q)}}=\frac{M_{w, S i O}}{M_{w, ~ S i}} C_{m g / L, S i}
$$

where $M_{1, i}$ is the molecular weight of the component labeled $i$. The atomic weight of $S i$ is 28.086 $\mathrm{g} / \mathrm{mole}$ and that of $\mathrm{O}$ is $15.999 \mathrm{~g} /$ mole. The molecular weight of $\mathrm{SiO}_{2(\mathrm{q})}$ is therefore 60.0840 $\mathrm{g} / \mathrm{mole}$. The ratio of the molecular weights is therefore 2.1393 , and the $8.52 \mathrm{mg} / \mathrm{L}$ of $S i$ is therefore equivalent to $18.23 \mathrm{mg} / \mathrm{L}$ of $\mathrm{SiO}_{2(\mathrm{aq})}$. By following this method, it can be shown that the $0.050 \mathrm{mg} / \mathrm{L}$ of $B$ is equivalent to $0.286 \mathrm{mg} / \mathrm{L}$ of $B(O H)_{3(a q)}$, and that the $0.210 \mathrm{mg} / \mathrm{L} \mathrm{of} P \mathrm{O}_{4}{ }^{3-}$ is equivalent to $0.212 \mathrm{mg} / \mathrm{l}$ of $\mathrm{HPO}_{4}{ }^{2-}$. Corrections are analogous if the data are concentrations in $\mathrm{mg} / \mathrm{kg}$ of solution.

The situation is much simpler if the analytical data are reported as molalities or molarities, as no conversion is generally necessary. For example, 0.0001 molal $S i$ is equivalent to 0.0001 molal $\mathrm{SiO}_{2(a q)}$.

The code user must make any necessary stoichiometric conversions before entering the data on the input file. EQ3NR contiins no provisions for direct input of data corresponding to dissolved components other than the basis species appearing on the data file used, so it is not possible for it to make these conversions for the user. 


\section{The EQ3NR Input File: Setting up the Problem}

\subsection{Input File Characteristics and Contents}

We examine in this chapter the EQ3NR input file. This file is the means by which the user specifies a problem to be solved by the code. The user must supply a compatible datal file, and the results obtained may differ if the problem is run with more than one such data file. Some examples of EQ3NR input files are presented in this chapter, and the reader will find more examples, along with the corresponding output files, in Chapter 7. Still more examples are given in Appendix F of the EQ6 Theoretical Manual and User's Guide (Wolery and Daveler, 1992).

The EQ3NR input file presently exists in one of two formats. The first is of the type used in previous versions of the code (e.g., Wolery, 1983). This is a compact form more suitable for use by experienced users. We will refer to this as the "W" format. A newer optional form (Daveler and Bourcier, unpublished) has a menu-style format that is much less compact, but which may be easier to use for less experienced users. We will refer to this as the " $D$ " format. The present manual will focus more on the " $W$ " format, as this naturally leads into a discussion of the relevant code variables. Some examples will be given of problems presented in both formats.

The recommended way of creating a new input file is to use an old one as a template. This works best if the old one is as close as possible to the desired new one. To this end, a representative selection of sample input files is included in the distribution package for EQ3/6 (Wolery and Daveler, 1992a). The distribution pa sage also includes some software which converts input files in "W" format to " $D$ " format. Using this template approach reduces the necessary knowledge a user must have conceming input file formats, and minimizes the need to consult the related documentation.

The "W" format input file is read by the code module readx.f. The source code for this module is extensively documented intemally by of comment lines. On-line access to the source code of this module may helpful to users, serving as a kind of on-line documentation. The " $D$ " format input file is read by the module rdninp.f, which calls a number of other modules in carrying out this function (the EQ3NR modules rdtyp1.f, rdtyp2.f, rd3tds.f, rdtyp4.f, rdtyp5.f, and rdtyp9.f, and the EQLIB modules rdtyp0.f, rdtyp6.f, rdtyp7.f, and rdtyp8.). The source codes of these modules may also be helpful to users as on-line documentation.

An input file of either format contains a title field to provide space for intemal documentation. In addition, an input file may contain remarks in comment lines. These are marked by an asterisk in column one and are analogous to comment lines in FORTRAN source code. They may appear anywhere in the input file.

Regardless of which format of input files is used, EQ3NR writes an "instant echo" of the input file on the output file. That is to say, after the code has read a line or closely related group of lines, it echoes their contents. This is particularly helpful in identifying the causes of read format errors, which most commonly occur when a line is missing or out of the proper sequence. This feature also provides a record of the input file used for a given run. However, it does not include comment lines. If necessary, a lost input file may be recovered (less comment lines) by extracting the echo from the output file. 
The user is cautioned that the number of lines in an EQ3NR input file in either format is variable. Whether or not some potential lines appesir in a given file deperds upon the contents of other lines. Some items, such as the constraints applied to the basis species, appear on successive lines until a terminator appears. Certain options, if invoked, require corresponding additional lines of input. The file structure bas been chosen so that the size of the input file reflects the size of the problem.

There are no species index numbers for users to bother with. Internally, EQ3NR sets up its own internal indexing schemes at run time. Users deal with species in terms of their names, which are 24-byte character variables. These are much easier to remember and are instantly recognizable. However, the names must match perfectly with those on the data file used or EQ3NR will nct recognize them. In such a case, the code will write an error message and terminate activity on the current problem input.

EQ3NR input problems may be stacked on the input file so that the code will read one problem, solve it, read another, solve it, and so on, in one job. In most cases, if an error is caught in one problem input, the code will proceed to the next problem input, if ahy.

A short summary of the contents of the EQ3NR input tile in "W" format is given in on the following pages. Parameters for which default values are recommended are marked with an asterisk. To take the defaidt for a given parameter, leave the corresponding input field blank. Following the short suminary is a discussion of the input file parameters themselves. The user need not $\mathrm{en}$ ter values for all of these. Some may not appear on specific examples of input files, depending on other parameters. This summary is followed by an example of an EQ3NR input file in both "W" and " $D$ " formats (more examples are given in Chapter 7).

Short Summary of the EQ3NR innut file ("W" format):

Parameters

Do $n$ from 1, ctuding with the string

'endit.' in colunn 1:

utill(n)

End do

tempe

rho, tdspke, tdspl

(emter only one of tdspkg, tdspl)

fep, uredox

(enter tiuler fep or uredox)

tollut(*), toldl(*), tolsal(*)

itermsx(*)

iopt1 - iopt 10
Format

(a80)

(12x,c12.5)

(3(12x,c12.5))

$(12 x, c 12.5,12 x, a 24)$

(3(12x,et2.5))

(12x,i2)

(12x,10i5) 


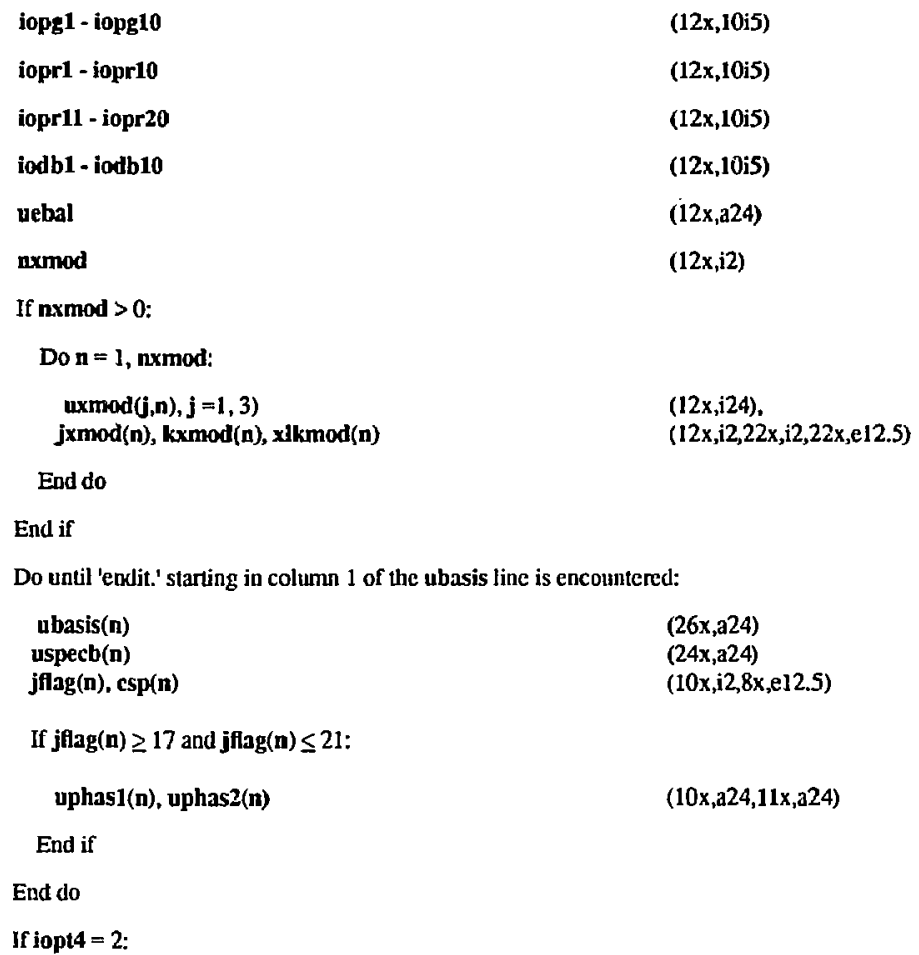

End if

Do until 'endit.' starting in column 1 of the ubasis line is encountered:
ubasis(n)
uspecb(n)
(26x,a24)
jflag(n), $\operatorname{csp}(n)$
$(24 x, a 24)$
$(10 \mathrm{x}, \mathrm{i} 2,8 \mathrm{x}, \mathrm{e} 12.5)$

If $\mathbf{j} f \operatorname{lag}(\mathbf{n}) \geq 17$ and $\mathbf{j f l a g}(\mathbf{n}) \leq 21:$

uphas1(n), uphas2(n)

$(10 x, a 24,11 \times, a 24)$

\section{End if}

End do

If iopt4 = 2:

Do until 'endit.' starting in column 4 of the usolb line is encountered:

usolb(n)

$(3 \mathrm{x}, \mathrm{a} 24)$

Do until 'endit.' starting in column 7 of the umemb line is encountered:

(umemb(i,n), xbarb(i,n) $\quad(5 x, a 24,3 x, f 10.4)$

End do

End do

End if

(*) Default values are recomnended (i.c., leave these blank on the input file) 


\section{Summary of EQ3NR input file parameters:}

utill Up to 100 lines of text that describe the ioput problem, teminated by an 'endit.' beginning in column 1.

tempc Temperature, ${ }^{\circ} \mathrm{C}$.

rho

Aqueous solution density, g/ml. 'ibe default value is 1.0 .

tdspkg Total dissolved salts, mg/:g of solution.

tdspl Total dissolved salts, $\mathrm{mg} / \mathrm{L}$ (do not enter both of tdspkg and tdspl).

Tep

Redox parameter.

$$
\begin{aligned}
& =p e \quad \text { if iopt1 }=-2 . \\
& =E h \quad \text { if iopt1 }=-1 . \\
& =\log \text { oxygen fugacity if iopt }=0 .
\end{aligned}
$$

uredox Name of an auxiiiary mas!er specics; this defines a redox couple that specifies the oxygen fugacily (iopt2 $=1$; cnter only one of fep, uredox).

tolbt Convergence toicrance in Newton-Raphson residual functions. The default value is $1 \times 10^{-6}$.

toldl Convergence tolerance, on Newton-Raphson correction terms. The default , we is $1 \times 10^{-6}$.

tolsat Saturation tolerance; this only determines whether or not a mineral is listed as "saturatea" on the output file; it bas no cffect on Newton-Raphson iteration. This value is used to test the affinity, not the saturation index. The default value is $0.5 \mathrm{kcal}$; reasonable values range from $0.1 \mathrm{kcal}$ to $1.0 \mathrm{kcal}$.

itermx Limit on the number of Newton-Raphson iterations. The default value is 30 .

iopt1 Option switch for cieternining the redox parameter input:

-3 = This option instructs the code to look for an input constraint on $O_{2 / g}$ is: a comal constraint block for a basis species (see uspecb, jflag, csp, uphasi, and uphas2 below; in this case, uspecb $\left.=^{\prime} 02(g)^{\prime}\right)$. By choosing jflag $=19,20$, or 21 , the oxygen fugacity can by fixed by a heterogenous reaction for a mineral, solid solution end-member component, or gas species, respectively (defined by the uphas1 and uphas? inputs, sec below).

$-2=$ The $p e$ is specified in fep.

$-1=$ The $E h$ is specified in fep.

$0=$ The log oxygen fugacity is specified in fep.

$1=$ An aqueous redox couple idemtified by the uredox variable constrains the oxygen fugacity.

iopt2 Option switch for automatic basis switching:

$0=$ Tunis it oft.

$1=$ Tums it on.

iopt3 Option switch for writing a pick up filc for illput to EQ6:

$-1=$ No pickup file is generated.

$0=$ A pickup file is generated. 
iopt4

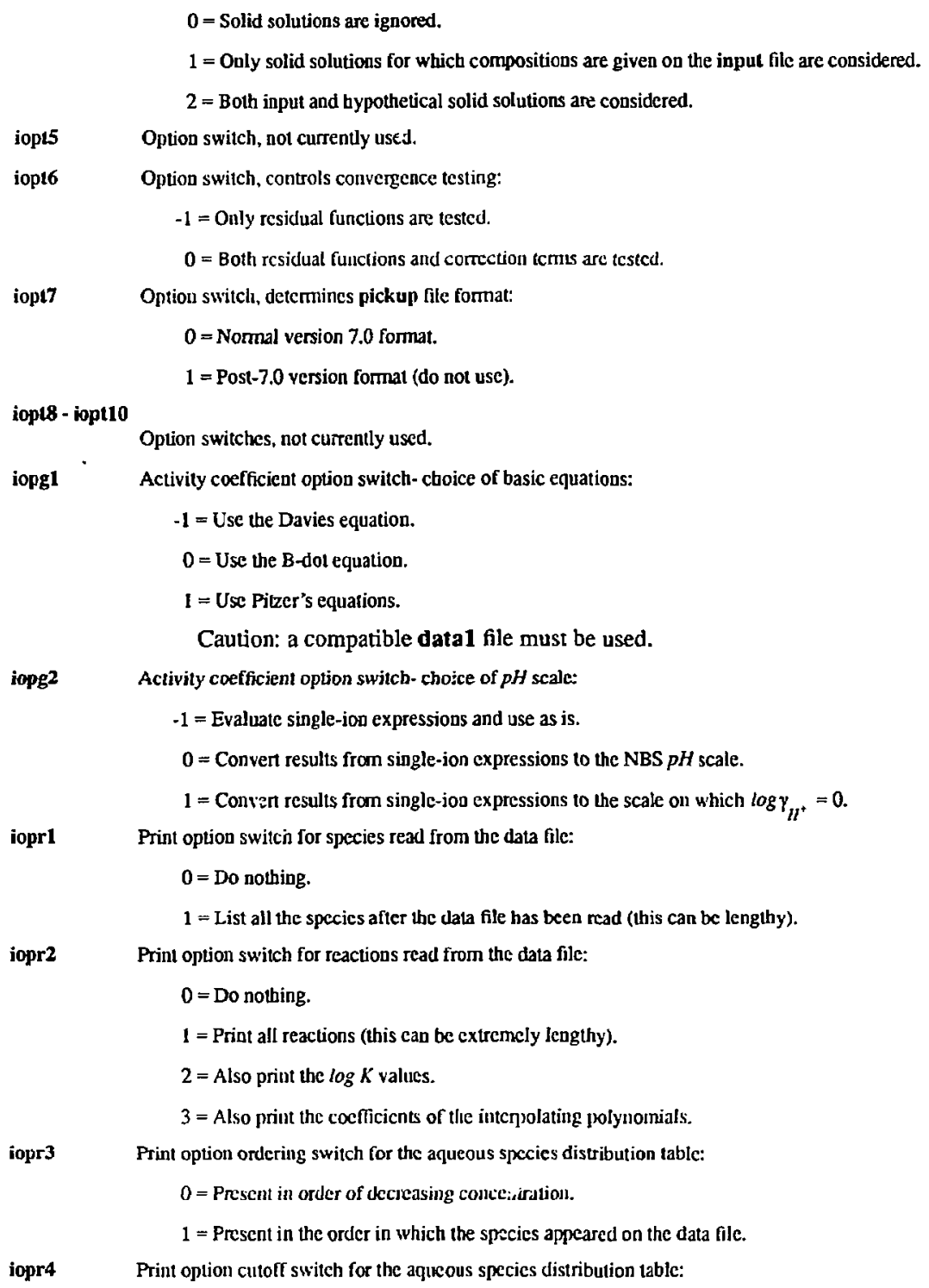


$0=$ Print all aqueous species.

$1=$ Print only those with concentrations greater than $1 \times 10^{-20}$ molal.

2 = Print only those with concentrations greater than $1 \times 10^{-12}$ molal.

$3=$ Do not print the aqueous species distribution.

iopr5 Print option cutoff switch for the mass balance percentage tables:

$-1=$ Do not print.

$0=$ Print, cutting off at $99 \%$ of the total for each table.

$1=$ Print all specics in cach table.

iopr6

Print option switch for the mean ionic properties table:

$0=$ Do nothing.

$I=$ Print the table.

iopr7 Print option for mineral affuity/saturation index tables:

$-1=$ Do not print.

$0=$ Print, cxcluding minerals whose affinities are less than $-10 \mathrm{kcal}$.

1 = Print data for all minerals.

iopr8 Print option for hard corc diameters and hydration numbers of aqueous species:

$-1=$ Do not print.

$0=$ Print, these data.

iopr9 Print option switch for Pitzer intcraction coefficients:

$0=$ Print only warnings, if any.

1 = Print the species in the model and the number of coefficients.

$2=$ Print the species and the associated pairs and triplets of species for which the coefficients are defined.

iopr10 Print option switch for the mcan ionic properties table:

$0=$ Do nothing.

$\mathrm{l}=$ Print the stoichiometric concentrations of the basis species.

iopr11 - iopr20

Print option switches, not currently used.

iodbl Debugging print option switch for general informational messages:

$0=$ Do nothing.

$1=$ Print certain messages which may be of diagnostic value.

2 = Pritt a lighler level of such messages.

iodb2

Dehugging print option switch for pre-Newton-Raphson optimization:

$0=$ Do nothing. 
$1=$ Print a summary of the optimization process.

2 = Print a more dctailed summary.

iodb3

Option switch for the iteration variable kill option:

$0=$ Do nothing.

$1=$ Invoke the killer option (this is intended for the usc of code developers only).

iodb4 Debugging print option switch for Newton-Raphson iteration:

$0=$ Print a sunumary of the process.

$1=$ Print a more detailed summary of the process.

iodb5

Debugging print option switch for stoichiometric cquivalence factors:

$0=$ Do nothing.

1 = Print the equivalence factors for oxygen and hydrogen mass balances only.

$2=$ Print the equivalence factors for all mass balances,

iodb6

Debugging print option switch for calculation of cquivalence factors:

$0=$ Do nothing.

I = Print dctails of thesc calculaticns.

iodb7 Debugging print option switen for reactions:

$0=$ Do nothing.

$1=$ Print the reactions on a file called rlist, before and after any hasis swilching operations (intended for use only by code devclopers).

indb8 - iodb10

Debugging print option switches, not currenty uscl.

uebal Name of ionic species for clectrical balancing; if uebal is 'pickl.', the codc picks a specics; if uebal is blank, no electrical balancing is done. In most instances, users should not have the code do elcctrical balarcing.

nxmod Number of alter/suppress options (number of species to be suppressed or whose $\log K$ values are to te modified).

uxmod Name of a species to be suppressed or wliose corresponding equilibrium constant is to be modified for use in the current nin.

$$
\begin{aligned}
& 0=\text { pure mineral. } \\
& 1=\text { solid solution. } \\
& 2=\text { spacial ractant. } \\
& 3=\text { acfucous species. } \\
& 4=\text { gas. }
\end{aligned}
$$

kxmod The uxnrod alter/suppress option (sec jxmod and uxmod): 


$$
\begin{aligned}
& -1=\text { Suppress. } \\
& 0=\text { Replace the } \log K \text { by xlkmod. } \\
& 1=\text { Augment the } \log K \text { by xlkmod units. } \\
& 2=\text { The } \log K \text { changed as if the corresponding Gibbs energy of reaction were } \\
& \text { decremented by xlkmod kcal/mole. }
\end{aligned}
$$

\begin{abstract}
xlkmod Equilibrium constant alteration function (see above).
ubasis The name of a nun-time basis species (if not the same as uspecb, the name of the species to switch into the basis sct in place of the specics whose name is input in uspecb).

uspecb The name of a data file basis species.

jflag The option flag which defines the type of input constraint (see below).

csp A floating point datum whose meaning is determined by the corresponding value of the jllag option flag; usually this is a concentration of some type.

uphas1 The name of an aqueous basis species or a mineral, solid solution, or gas species required to define an input constraint under the jflag $=17,18,19,20$, and 21 options.

uphas2 The name of solid solution end-member component required to defite an inpul constraint under the jllag $=20$ option.

usolb The name of a solid solution for which a composition is entered for the purpose of computing the corresponding affinity and saturation index.

umemb The name of an end-menber component of the solid-solution whose name is input in usolb.

xbarb The mole fraction of a solid solution cnd-member component whose name is input in umemb.
\end{abstract}

\title{
Table of iflag Options:
}

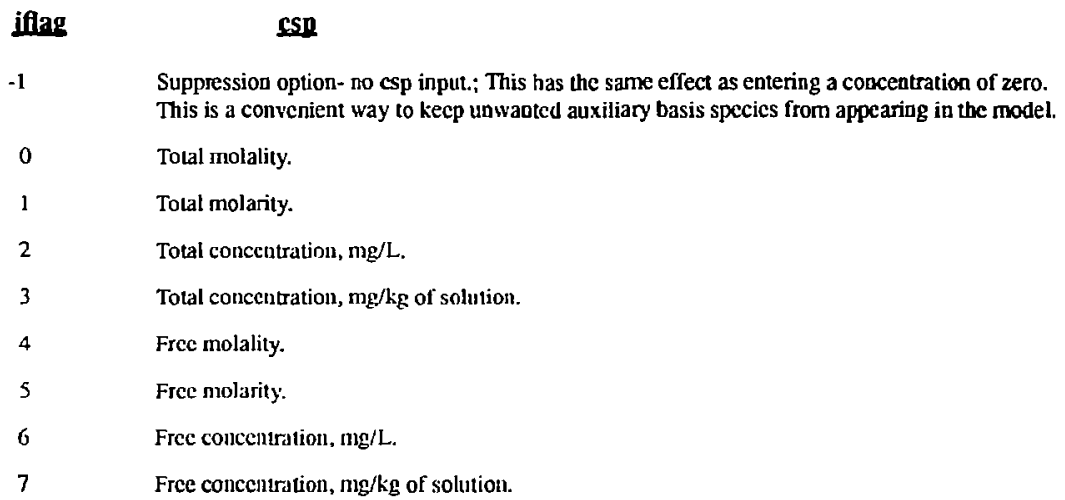


Free concentration, $\mathrm{cm}^{3}(\mathrm{STP}) / \mathrm{cm}^{3}$ solution.

The $\log$ activity. This is the means of entering $p H\left(-p H=\log a_{H^{+}}\right)$.

Combination $\log$ activity function-enter the name of the corresponding ion as

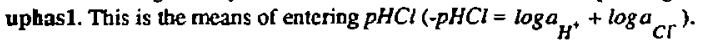

Mean log activity- enter name of corresponding ion as uphas1.

19

Equilibrium with a pere mineral- no csp input; enter name of mineral as uphas1.

Equilibrium with a solid solution end-menber component- no csp input; enter name of the solid solution as uphas1 and the name of the end member as uphasz. put; this option is available only for auxiliary basis species. This is the default for $O_{2(a q)}$ and $H_{\text {Trags }}$

\section{Example of an EQ3NR input file in "W" format.}

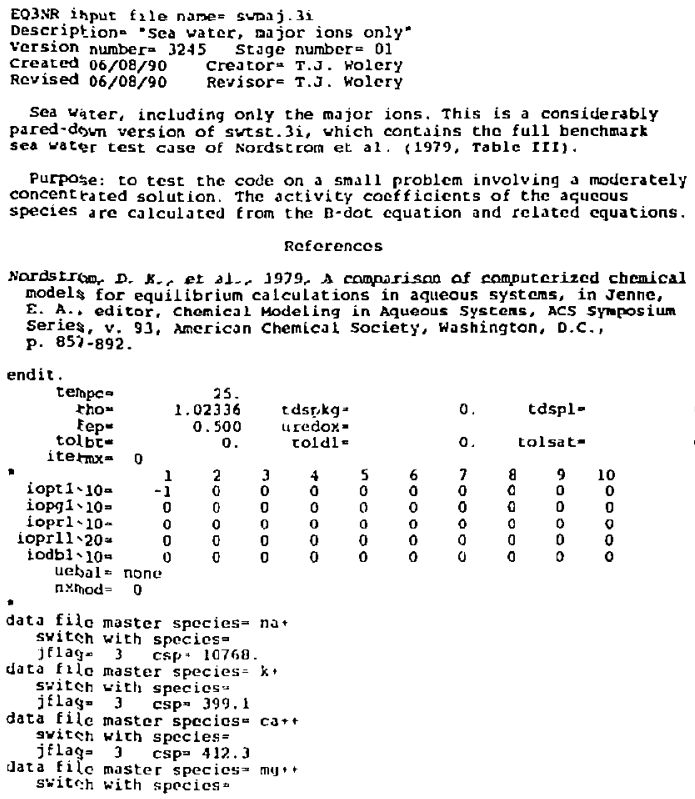




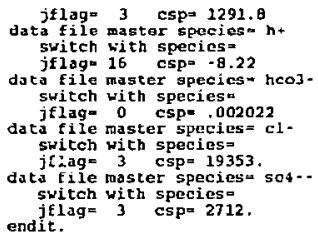

The EQ3NR input file in " $D$ " format is illustrated by the following example, which contains the same problem that was just presented in " $\mathrm{W}$ " format. The mapping between the two is largely self-evident. The most immediately obvious characteristic of an input file in " $D$ " format is its use of the " $\mid$ " character to delimit fields in which data are entered. Note that an asterisk ("*") is used to mark the choice of units for entering total dissolved salts. It may also be used to choose among two of the options for electrical balancing ("code selects" and "nat performed"). If electrical balancing is to be done on a specified ion, the name of the ion should simply be entered in the field provided. The principal option switches are set in blocks in which an asterisk is used to mark the desired choice. If more than one choice is marked for the same option, the code writes an error message and execution stops. In the case of debugging option switches, the user enters a numerical input of 0,1 , or 2 . If 2 is a valid input, a "2" appears on the line following the final "s". When $E Q 3 N R$ reads an input file in " $D$ " format, it immediately checks the range limits and notes discrepancies in the instant echo on the output file. When the code reads an input file in "W" format, such checks are made after the input file has been read.

Note that the iopt1 option is handled in a special way. 1t does not appear in the options block, but in the block of inputs for the basis species. Here it appears as a "species" called 'REDOX'. This is used in the example to input the $E h$ by entering the corresponding string 'EH'. The available options are as follows:

\begin{tabular}{|c|c|c|}
\hline iont 1 & "D" format string & Meaning \\
\hline-3 & 'REDOX COUPLE' & $\begin{array}{l}\text { A nomal hasis specics input line for } \mathrm{O}_{2 \mathrm{rg}} \text { is expected to } \\
\text { immediately follow the 'REDOX' input line. The "D" format } \\
\text { string for this option is not descriptive of the actual option; } \\
\text { scnething like 'O2 INPUT" would have been a better choice. }\end{array}$ \\
\hline-2 & 'PE' & pe. \\
\hline-1 & 'EH' & $E h$, volts. \\
\hline 0 & 'LOGFO2' & log oxygen fugacity. \\
\hline 1 & 'REDOX COUPLE' & $\begin{array}{l}\text { Use redox couple defincd by uredox input. The auxiliary basis } \\
\text { species correspondling to the uredox variable is defined by } \\
\text { the species on the species input line immediatec', following the } \\
\text { 'REDOX' input line. It is not defined in the constraint field } \\
\text { on the 'REDOX' input line itself, as one might expect. }\end{array}$ \\
\hline
\end{tabular}

Although these strings are listed here in upper case, the code does not consider case in interpreting them. Similar strings noted below are treated in the same manner. 
Instead of entering a kxmod number to define the type of an "nxmod" alter/suppress option, one uses one of the following strings:

\begin{tabular}{lll} 
Kxmod 'D" format string & \multicolumn{1}{c}{ Meaning } \\
\cline { 2 - 3 }-1 & 'SUPPRESS' & Suppress the phase/reaction. \\
0 & 'REPLACE' & Replace the $\log K$. \\
1 & 'AUGMFNTK' & Augment the $\log K$. \\
2 & 'AUGMENTG' & $\begin{array}{l}\text { The log } K \text { changed as if the corresponding Gibbs energy of reaction } \\
\text { were decremented by xlkmod kcal/mole. }\end{array}$
\end{tabular}

The jflag options are also handled in " $\mathrm{D}$ " format by character saings. These are listed in the following table.

\begin{tabular}{|c|c|}
\hline ifigg & "D" format string \\
\hline 0 & 'MOLALITY' \\
\hline 1 & 'MOLARITY' \\
\hline 2 & 'MG/L' \\
\hline 3 & 'MG/KG' \\
\hline 4 & 'FREE MOLAL' \\
\hline$s$ & 'FREE MOLAR' \\
\hline 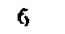 & 'FREE M:Ts/L' \\
\hline 7 & 'FREE MG/KG' \\
\hline 8 & 'FREE CM3/CM' (*) \\
\hline $1 \epsilon$ & 'LOG ACTIVITY $\left(\frac{1}{\dagger}\right)$ \\
\hline 17 & LOG ACTIVITY COMBO (††) \\
\hline 18 & 'LOG MEAN ACTIVITY' \\
\hline 19 & 'MINERAL' \\
\hline 20 & 'SOLID SOLUTION' \\
\hline 21 & 'GAS' \\
\hline 27 & 'DEPENDENT' \\
\hline 30 & 'ELIMINATED' \\
\hline
\end{tabular}

(*) This is a typographical error in the code.

(i) One may use the string 'PH' 10 enter the $p H$.

(iti) One may use the string 'PHCL' to enter the $p H C l$. 


\section{Example of the same EQ3NR input file in " $D$ " format.}

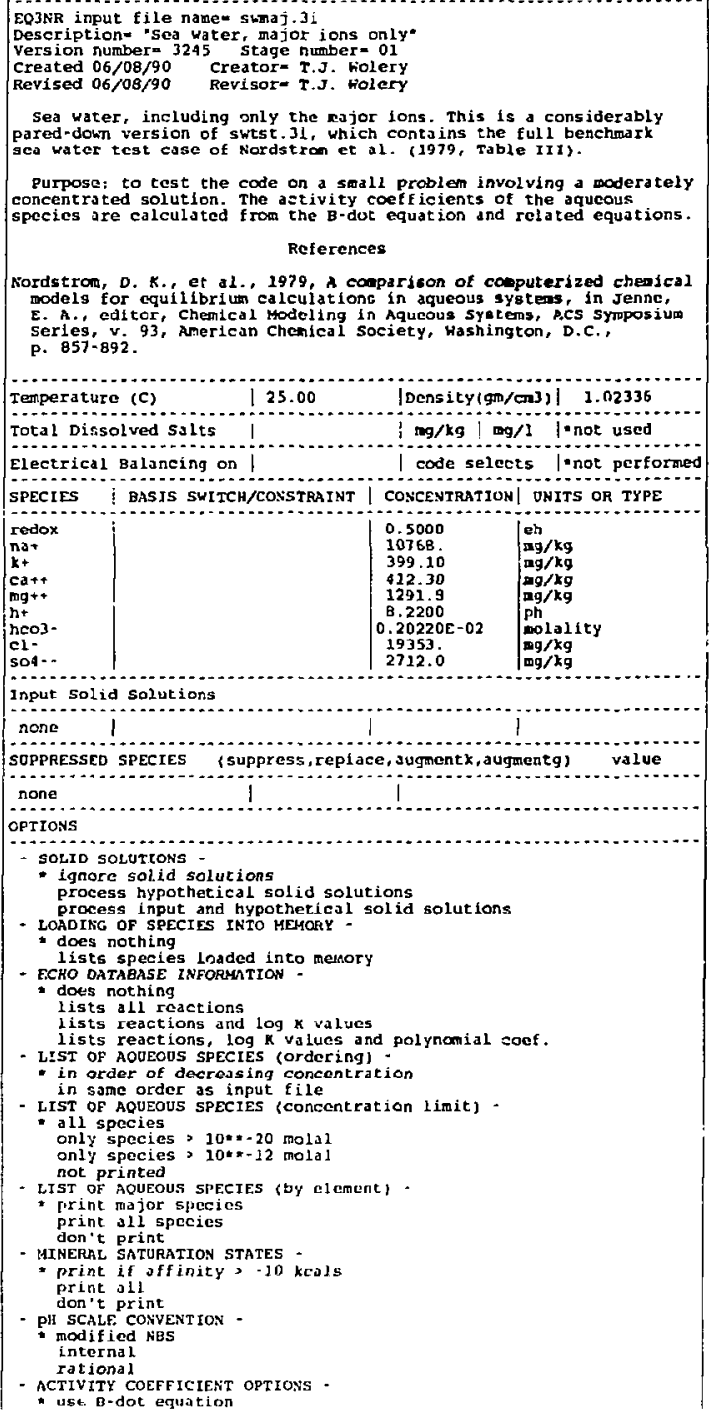

sea water, including only the eajor fons. This is d considerably pared-dohn version of swtst.31, which contains the full benchmark geo water test case of Nordstron et al. (1979, sable III).

purpose; to cest the code on a swall problem involving a moderately concentrated solution. The activity coetficients of the aqueaus species are calculated from the B-dot equation and related equations.

References

Nordstrom, D. K., et al., 1979, a conparicon of cobputerized chenjcal models for equilibriun ealculations in aqueous systens, in Jennc, E. A., editor, Chemlcal todeling in Aqueous syetems, p.cs syroposium serles, v. 93, Amerlcan Chenical society, Hashington, D.C., p. $857-892$.

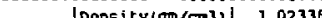

Total Dissolved salts |
Electrical Balaneing on |
SPECIES DASIS SWITCH/CONSTRAINT | COSCENTRATION| ONITS OR TYPE

\begin{tabular}{|c|c|c|}
\hline $\begin{array}{l}\text { redox } \\
\text { nat } \\
\text { ht } \\
\text { catt } \\
\text { mgt+ } \\
\text { ht } \\
\text { heos. } \\
\text { cl- } \\
\text { sot-. }\end{array}$ & \begin{tabular}{|l}
0.5000 \\
10768 \\
399.10 \\
412.30 \\
1291.9 \\
0.2200 \\
$0.20220 \mathrm{E}-02$ \\
19353. \\
2712.0
\end{tabular} & 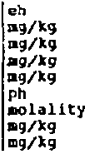 \\
\hline
\end{tabular}

Input solid solutions

none |

SOPPRESSEd SPECIES (suppress, repiace, augnentx, augmentq) value

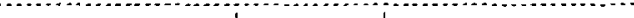

none |

OPTIONS

Solid solutrons

- ignore solid solutions

process hypothetical solid solutions

orocess input and hypotherical solid solutions

LOADIKG OF SPECIES INTO HEHOFY

does nothing

lists species Loaded into menory

- Ecko database lNFormtion

- does nothing

lists all reactions

lists reactions and log $x$ values

lists reactions, $\log K v_{a}$ lucs and polynomial soef.

LIST OP AOUEOUS SPECIES (ordering)

- in order of decreasing concentration

in same order as input $f$ ile

- LIST OF AQUEOUS SPECIES (concentration limit).

- all species

only species > 10*:20 molal

only species > 10*-12 mola

not printed

- LIST OF AOUEOUS SPECIES (by element)

* rirint major species

print all species

don't print

- uneral saturation states

- priat if offinity> - 0 kcols

print all

don't print

- pH scale convent Ion -

- modified NBS

internal

rational

- ACTIVITY COEFFICIENT OPTIONS

- ust. B-dot equation 


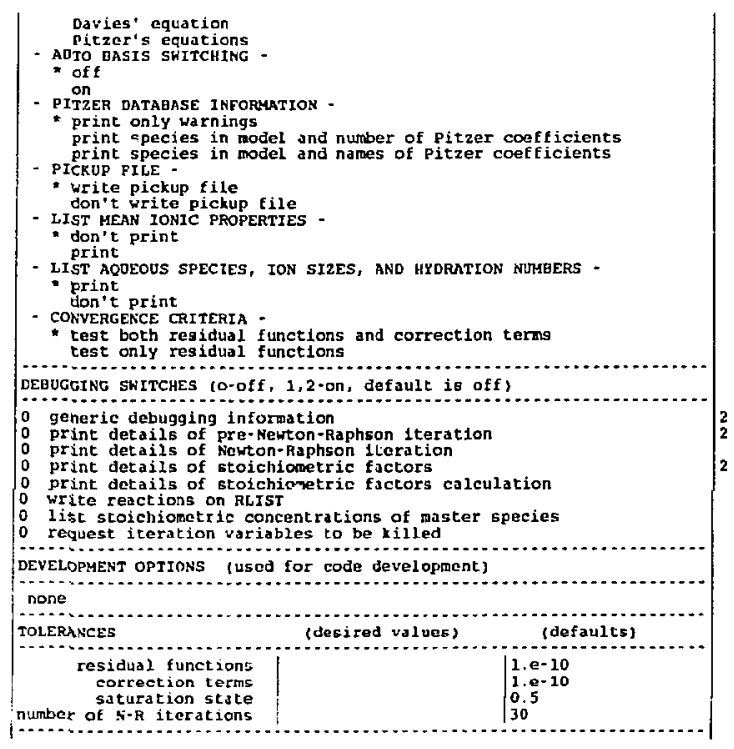

EQ3NR input files in " $D$ " format are treated differently from ones in "W" format in one important way. The input from files in " $D$ " format are checked against allowed range limits, where such exist, as soon as the relevant lines of data are read. Error or waming messages may therefore appear right after these lines in the instant echo portion of the output file. Essentially the same checks are made for input in "W" format, but after the current problem has been read from the input file. Any relevant error or waming messages therefore appear after the instant echo on the output file.

\subsection{Cautions}

In the absence of analytical data, it is recommended that users take the default condition of jfiag $=27$ for the auxiliary basis species $O_{2(a q)}$ and $H_{2(a q)}$. The reason is that one (or the other) will then ustia. y have a low but sufficiently high concentration to insure some poising of the oxygen fugacity. This has little significance if one is not passing the solution model on to EQ6. However, if one does, this is helpful in avoiding computational difficulties in that code which may arise in trying to treat very ill-poised systems. As an example, a user might be interested in the dissolution of a feldspar in $\mathrm{CO}_{2}$-charged water. This problem appears to have no redox aspect, but EQ6 expects a problem to have a redox aspect unless the user invokes special options. If some $\mathrm{O}_{2}(\mathrm{aq})$ is not present in the modeled solution and these special options are not invoked, the code will un with very small step sizes, uying to do the nearly impossible job of accurately calculating the oxygen fugacity when there is hardly anything present to define it. If on running EQ3NR one sets iopt $1=0$ and sets fep to -0.700 , the oxygen fugacity will at the atmospheric value and a concen- 
tration of $\mathrm{O}_{2(a q)}$ will be computed which is in equilibrium with this. This is encugh to poise the system in the example cited, and EQ6 can then be run quite nicely without having to invoke the above noted siecial options.

If the user creates an aqueous system model in which data are input for an auxiliary basis species but not for the corresponding strict basis species, the results from the code calculation may not be what the user intended. Suppose an analyst reports $2 \mathrm{mg} / \mathrm{L}$ of dissolved $F e$ for some water. On all of the existing EQ3/6 data files, $\mathrm{Fe}^{2+}$ is the strict basis species and $\mathrm{Fe}^{3+}$ is in the auxiliary basis. Generally speaking, the quantity reported by the analyst really means total dissolved iron of either form. To input this correctly, the user must enter $2 \mathrm{mg} / \mathrm{L}$ for $F e^{2+}$. Then letting jflag for $\mathrm{Fe}^{3+}$ default to 30 , the mass balances for the two forms are tombined and the calculation is done correctly. On the other hand, if the user inputs $2 \mathrm{mg} / \mathrm{L}$ for $F e^{3+}$, the jflag value for $F e^{2+}$ will default to -1 , an intermal value equivalent to jflag $=0$ and $\operatorname{csp}=0$. In other words, $F e^{2+}$ (and its associated ion pairs and complexes) will be absent from the model, and the input total concentration will be disurbuted only among $\mathrm{Fe}^{3+}$ and its ion pairs and complexes.

As a general rule, it is not wise to create EQ3NR models in which an auxiliary basis species is present in the absence of the corresponding strict basis species. The calculations may be valid. However, the resulting acpuenus solution model can not be input to EQ6. In situations in which an auxiliary basis species is present in the actual absence of the corresponding strict basis species, the roles of these species should be reversed by a basis switch. The instructions for the switch and the desired jflag and csp values should be entered on the input file as input for the original strict basis species. As the csp input is initially interpreted in terms of this species, and will then be recalculated by the code for stoichiometric equivalence with the species switched into the strict basis set, it may be necessary to recalculate the analytical data for stoichiometric equivalence with the original basis species. The code will then invert this same calculation when it makes the basis switch. 


\section{Sample Problems: Inputs and Outputs}

\subsection{Introduction}

This chapter presents the input and output files for several speciation-solubility modeling problems that are successfully executed by EQ3NR. Each example begins with a short discussion. The input file and output files are then presented. The reader is encouraged to compare the input file examples presented here with the input file description presented in Chapter 6 . Note that each output file begins with an "instant" echo of the input file. The examples presented here were run on a Sun SPARCstation IPC using optimized code, using the "W" format input files and the "R 10 " com and $\mathbf{h m w}$ data files. More examples of EQ3NR input files can be found in Appendix F of the EQ6 Theoretical Manual and User's Guide (Wolery and Daveler, 1992). The examples presented here are relatively simple. Two input files addressing more complex problen's are presented without external comments or computed results in Appendix E.

On the output files one will occasionally sec "+999" and "-999". These are respectively treated in EQ3/6 as the logarithms of plus infinity and zero, respectively. In the context of their appearance in these files, they generally signify a condition of "no datta.".

\subsection{Sea Water Test Case, with Major Cations and Anions Only}

The first simple problem is the sea water test case from Nordsurom et al. (1979), but in which only the major cations and anions are included. This simplified test case is a classic exampie in geochemistry (Garrels and Thompson, 1962; Garrels and Christ, 1965). The input file was presented in both " $W$ " and " $D$ " formats in the previous chapter, and will not be repeated here. We will here focus on the output $f$ le. The corresponding pickup file is presented in Chapter 8 . The data 1 file used is the com file, and the activity coefficients sue computed from the B-dot espuation (iopg1 =0).

The output file for this problen is presented in its entirety. The output begins with the name of the code and the version identification ("EQ3NR, version 3245.1090R124"). 1 his is followed by a copyright notice and a set of disclaimers. This in turn is followed by at time and data stamp ("Run 09:31 3Dec91").

The instant ccho of the input file appeas following the line, "- - reading the input file -. -". The input file contents appeat shifted one character to the right, to be consistent with the use of older canjage control conventions. If all goes well this is followed by a blank line and the message.".-- the input file has been successfully read -...".

The next alction of the code is to sead the datal file. Note thit the code prints messiages when it starts (". - reading the datal file -..") and finishes ("-.- the datal file has been successfully read -.-") this action.

This is followed by "EQ3NR"in layge block letters. The code name and version identification are given. allong with the version identification for the EQLIB library. The title from the injut tile is printed, followed by the name and version identification of the supporting dita file. Problent and data file statistics are then printed. followed by a list of the problem inputs, including any default values or truncated values taken by the code. The table appearing under the header 
"-- - input constraints - - " is part of this summary. It is illowed by a table headed by "-- - inactive aqueous species -- -". The entries in this table, if any, corre spond to species which are either lacking thermodynamic data or have been suppressed by user options on the input file.

This is followed by the rable headed by "-..- modified input constraints -..". This lists the model inputs as they have been modified by the program before Newton-Raphson iteration commences. The modified constraints may differ from the original ones in several ways. They include all conversions of concentration units to the molal scale, any defaults provided by the program, and the effects of any basis switching. This table also shows the status of any auxiliary basis species that pertain to the model but which were not listed on the input file. Users should make it a point to examine this table to ensure that the model they are getting is indeed the one they want.

At this point, the code has set up the problem and is ready to solve it. The code then sets up statting estimates and refines them somewhat according to a pre-Newton-Raphson optimization algorithm described in Chapter 9. If this is successful, the code writes, "-.- optimization ended within requested limits ---". This step is not always successîul, which leads to the appearance of a message to that effect. However, this does not mean that the code has or will fail to solve the problem. It only means that the optimization algorithm failed to satisfy a set of tolerances before handing the problem over to hybrid Newton-Raphson iteration.

Some data are then printed shc wing the progress of hybrid Newton-Raphson iteration. The user does not need to be concerned with these data as long as the iteration process succeeds. The printing of these data on the output fle is primarily done to provide an obvious break between the problem setup and the problem results. If iteration is successful, a message is printed to that effect ("Hybrid newton-raphson iteration converged in 4 steps.").

The data that follow should all be self-explanatory. Note that in the table headed by " distribution of aqueous species -..." many organic species appear. This occurs because the code is treating these species as complexes of $\mathrm{HCO}_{3}{ }^{-}$. This problem was discussed in Chapter 5 (at the end of Section 5.2). The R10 versions of the com and sup data files have a small number of organic species are in the auxiliary basis. There is a larger number of nonbasis organic species, which are related by their associated reactions to these auxiliary basis species. These in turn are each related to $\mathrm{HCO}_{3}{ }^{-}$. Looking in the table headed by " - - modified input constraints - - -", we see what the species are and that their jflag values have been assigned default values of 30 . This eliminates them from the active basis set and causes them and all related non-basis organic species to be treated as complexes of $\mathrm{HCO}_{3}$.

Knowing what these auxiliary basis species are, one could go back and enter $\mathbf{j f l a g}=\mathbf{0}$ and $\mathbf{e s p}=$ 0 for each of them, and then no organic species would appear in the computed model, which is really what one might normally desire. However, in this case, the results are not sensibly different from those that were desired anywily. Because the oxygen fugacity is high, the computed concentrations of all of these organic species are vanishingly small from a practical point of view. 
Organic species can be more easily kept out of a speciation model using the newer RI6 data files. The species 'acetic acid(aq)', one of the organics in the auxiliary basis, is now treated as the "mother" of all other organics. This was accomplished by writing the reactions of the other organic auxiliary basis species so that these species are converted to aceic acid instead of bicarbonate. Thus, entering $\mathrm{jftag}=0$ and $\operatorname{csp}=0$ for acetic acid suffices to prevent the appearance of any organic species (caution: the species $\mathrm{CH}_{4(a q)}$ is not treated as an organic; enter jflag $=0$ and $\operatorname{csp}=0$ for it as well if this species is not desired). Similarly, on the R16 data files, the species $\mathrm{S}_{2}{ }^{2-}$ is the "mother" of other polysulfide species such as $\mathrm{S}_{3}{ }^{2-}$ and $\mathrm{S}_{4}{ }^{2-}$, and $\mathrm{S}_{2} \mathrm{O}_{3}{ }^{2-}$ is the "mother" of similar partially oxidized sulfur species such as $\mathrm{S}_{2} \mathrm{O}_{4}{ }^{2-}$ and $\mathrm{S}_{2} \mathrm{O}_{5}{ }^{2-}$. The R16 data files became available close to the publication deadline for this series of reports. It was not feasible to update the examples using these data files.

In the "---- summaxy of aqueous redox reactions -----", we see that the tabulated redox parameters have the same values for all of the redox couples in the table. This indicates that all these couples are in mutual equilibrium. This is not a scientific conclusion about the chemistry of sea water. Rather, it is an example that what comes out of a model must be consistent with what went into it. The input constraints for this problem assumed this equilibrium.

In the "...-- summary of stoichiometric mineral saturation states - ...", note that low to moderate supersaturations are predicted for several carbonate minerals, including calcite and aragonite. These are to be expected for surfaces sea water at $25^{\circ} \mathrm{C}$ (see for example, Berner, 1971).

Note that when the code is done with this problem, it looks for another on the input file. Not finding any, it terminates by writing run time statistics.

The EQ3NR outout file for the seal water test case:

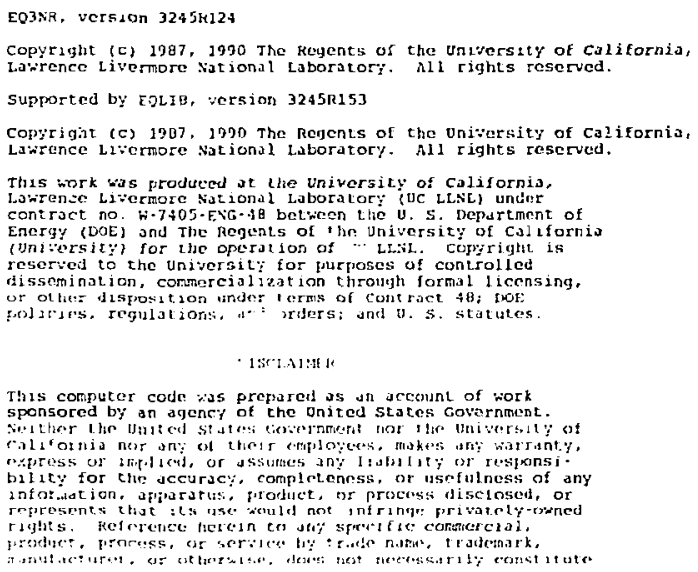


or imply its endorsetuent, recomendation, or favoring by the

united states Government or the oniversity of California

The vieus and opinions of authors expressed herein do no

necessarily state or reflect those of the onited states

government or the oniversity of Californiar and shall not

be used for advertising or product endorscent purposes.

LLNL YOCCA HOUNTAIN PROJECT DISCLAIMER

The Lawrence Livermore National Laboratory, a participant in

Che Yucca sountain Project, has not determined that this

coftware constitutes "approved code" for the conduct of

"quality affecting work" for the Yucea Mountain Project.

Fun $09: 31$ 3Dec91

-. reading the input file $\cdots$

EQ3NR input file somea simaj. 31

Deseription= "Sca water, major lons only"

Version number= 3245 stage number= 03

created $05 / 08 / 90^{32}$ Creator T.J. Wolery

Revised 06/08/90 Revisor T.J. Wolery

sca water, including only tho mojor lons. Thts is a considerably pared-down version of swtst, 31 , which cpntaing the full benchmark sea water test case of Nordstrom et al. (1979, Table III).

Purfoge: to test the code on a small problem involving a moderately concentrated solution. The activity coefficients of the aqueous species are calculated from tho p-dot equation and related equations.

ReE erences

Nordstrom, D. K., et al., 1979, A comparison of computerized chenical models for equilibrium calculations in aqueous systens, in Jenne,

E. A., editor, Chemical Modeling in Aqucous systers, ACs Symposium Series, $v, 93$. Anerican Chemical society, washington, $0 . c$.

p. $857+892$

endit.

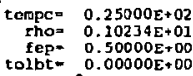

temper $0.25000 E+02$

Thos $0.20234 \mathrm{E}+01$

fep- $0.50000 \mathrm{E}+00$

tolbt- $0.00000 E+00$

tdspkg= $0.00000 \mathrm{E}+00$

uredox-

toldl= $0.00000 E+00$

cdspl- $0.00000 E+00$

itermx $=0$

1opt1-10

iopg1-10=

iopr1-10.

iopr 11-20*

iodbl-10*

uebala none

nxmod= 0

data file master speciese nat

switch with speciese

jflug= 3 esp= 0.10768E+05

data file master species" $k$ +

switeh with species

jflage 3 espa $0.39910 E+03$

data file master species. cat+

switch $j$ ith speries=
jflag= 3 csp= $0.41230 E+03$

data file master species. mgt+

switch with species"

jflag= 3 csp $0.1291 \mathrm{BE}+04$

data file master species= $h t$

switch with species=

iflaga 16 csp $=-0.82200 E+02$

data file master speciese heo3-

switch with species.

jelag $=0$ cspa $0.20220 \varepsilon-02$

data file master species- $\mathrm{cl}$ -

switch with species-

jelag= 3 csp= $0.19353 \mathrm{E}+05$

data file master spccies" sot-

switeh with species

endit

if $1 \mathrm{ag}=3 \mathrm{csp}=0.27120 \mathrm{0}+04$

-.. the input file has been successfully read -.

-. reading the daca $\downarrow$ flie $\cdots$

-. the datal file has been successfully read ...

- note - (eqlib/inbuot) The folloying anucous species

have been assigned a lofoult hard core diameter of 4.000 Angst caclo (an) 


\section{cacol (aq)}

casos(ag)

$\because=2$ (aq)

khsou (aq)

koh (aq)

$59 \cos (\mathrm{aq})$

nach 3 coo (aq)

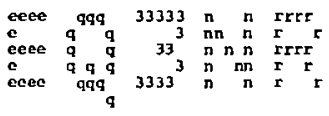

equnr, 3245k124x

supported by eq11b.3245R153

EO3NR input fllo name= shrmaj.3j

Deseription= "Sca water, tajor ions only"

Versior number- 3245 stage nunber= 01

$\begin{array}{ll}\text { Created } 06 / 08 / 90 & \text { Crcator } \\ \text { Revised } 06 \% 08 \% 90 & \text { Revisor T.J. Holery }\end{array}$

sea water, tncluding only the major ions. ThIs is a considerably pured-dotr version of gutst. $3 i$, which contains the full benchmark sea vater test case of Nordstron et al. (1979. Table III).

Purpose: to test the code on a small problen involving a moderately concentrated solution. The activicy coefficients of the aqueous species are calculated fran the B-dot equation and related equations.

\section{References}

Nordstrom, D. K. et al., 1979, n comparison of computerized chemical models for equilibrius calculations in aqueous systems, in Jenne,

E. A.. editor. Chemical Hodeling in Aqueous systems, ACS Sympasium

series, $v$. 93, Anerican Chenical Society, Washington, D.C.,

p. $657-892$.

data $0 . \cos . \mathbf{R} 10$

THERYODYNAMIC DATAEASE

generated by gemboens/INGRES $15-\mathrm{apr}-91$

geNerated by gembers/INGREs 15 -apr-91

the activity coefficients of aqueous solute species and the activity of water are calculated accorditig to the b-dot equation plus others

temperature 25.00 degrees celsius pressuree 1.0132 bars

78 elements are in the data base

100 elements can be loaded into manory

9 elements are active in this problat

852 aqueous species are in the data base

258 aqueous species were loaded into momory

800 aqueous species can be loaded into merrory

133 aqueous species are active in this problem

773 aqueous reactions are in the data base

179 aqueous reactions vere loaded into menory

699 aqueous reactions can be loaded into tremor;

886 minerals are in the data base

87 mincruis werc loaded into memor:

Q50 mineruls can be loaded into meriory

Of minerals are active in this problem

12 solid solutions are in the dutu base

so solid solutions can be loaded into memory

76 yases are in the duta base

16 plases tere loaded inco memor:

00 gases can be loaded into memory

16 gases are octive in this problen 


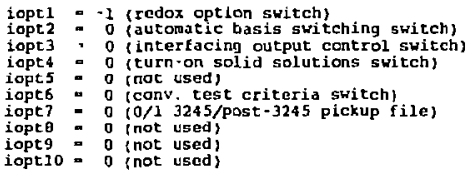

lOpr $=0$ llist losding of spectes

lopr2 - o (list reactions and $10 \mathrm{l} x$ values)

lopry a 0 (aqueous species print order control

iopra - 0 (aqueous species print cut-off control)

iopr5 - 0 (noss balance percentages print control)

iopr6 - 0 (noon ionic act coeff print control)

LOpr 7 a (mijeral affinity print control)

lopr 8 - 0 (ion size and hydr. no. print control)

ioprg a 0 (pitzer coefficients tabulation?

Loprio = 0 (Print concbs array)

lopril -0 (not used)

iopr 12 - 0 (not used)

iopr13 = 0 (not used)

iopr $4=0$ (not used)

iopr $14=0$ inot uscd

o nat uscd

o rnot used

lopr17 - 0 (not used)

iopr18 $=0$ (not used

iopr I9 $=0$ (not used)

iopr20 $\quad 0$ (not used)

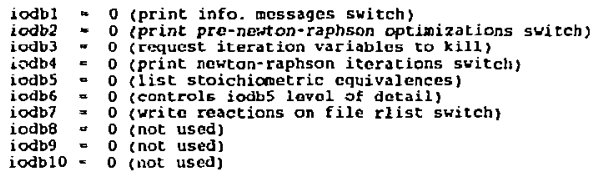

solution density $=1.02336 \mathrm{~g} / \mathrm{hl}$

total dissolved salts $=0.00 \mathrm{mg} / \mathrm{kg}$ solution

totol dissolved salts = $0.00 \mathrm{mg} / \mathrm{l}$

tolbt - 0.10000 E 05 (convergence tolerance on residual functions)

toldt a 0.10000E-05 (convergence tolerance on correction cerms)

tolsat = $0.50000 E+00$ (phase soturation tolerance, does not affect convergence

.. input constraints -.-

species

$$
\operatorname{csp}
$$

je 239

inpuc type/co-species

nu+

c.

$\mathrm{ca}$

fhit

heos-

heos-

so. -

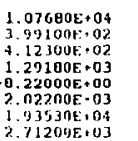

tot conc, mg/kg

tot cone, mos $k$ is

tot conc, nis/kij

tot conc, ng $/ \mathrm{kg}$

log activity

cot conc, mols

tot cane, nig/kg

tot conc, taig/kis

.. inactive acueous species ...

(o-phin)..

thenzenceads 
-.. modified input constraints ...

species

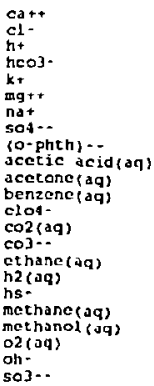

$\operatorname{csp}$

1. $02874 \mathrm{E}-02$

5.45BB2E-01

$-8.22000 E+00$

$2.02200 E-03$

$1.02076 \mathrm{E}-02$

$5.31496 \mathrm{E}-02$

4.68382E-01

$2.82313 \mathrm{E}-02$

$0.00000 E+00$

$0.00000 E+00$

$0.00000 \mathrm{E}+00$

$0.00000 \mathrm{E}+00$

$0.00000 E+00$

$0.00000 E+00$

$0.000 C O E+00$
$0.00000 E+00$

$0.00000 E+00$
$0.00000 E+00$

$0.00000 E+00$

$0.00000 E+00$

$0.00000 \mathrm{E}+00$

$0.00000 E+00$

$0.00000 \mathrm{E}+00$
$0.00000 \mathrm{E}+00$

$0.00000 \mathrm{E}+00$

$0.00000 E+00$ fflag input rype/co-spectes

cot cone, molal

tat canc, molal

log activity

tot conc, molal

tot conc, mola

tot canc, molal

tot cone, molal

tot conc, molal

eliminaced species

eliminated specics

climinated species

climinated specics

climinated specics

eliminated species

climinated species

eljminated species

dependent species

oliminated species

climinated species

climinated species

climinaeed specics

dependent spoe

climinated specios

climinated species

.. optimizdtion ended within requested limics -..

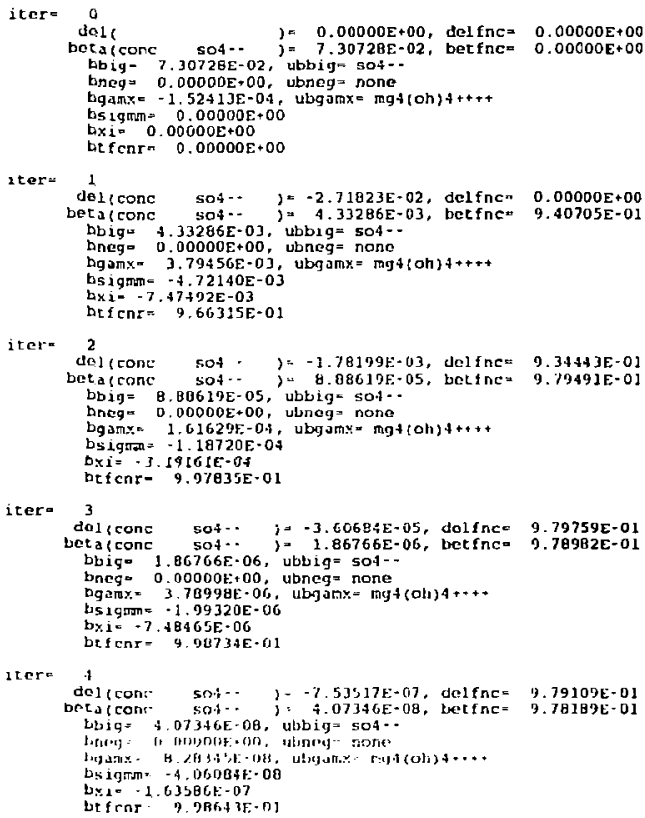


Elemental composition of the aqueous phase .....

\begin{tabular}{|c|c|c|c|}
\hline lement & $\log / 1$ & $\mathrm{mg} / \mathrm{kg}$ & moles/kg \\
\hline $\begin{array}{l}o \\
\text { ca } \\
c 1 \\
h \\
c \\
\text { m } \\
\text { mg } \\
\text { na } \\
\text { s }\end{array}$ & $\begin{array}{l}0.91080 \mathrm{E}+06 \\
121.93 \\
19805 \\
0.11451 \mathrm{E}+06 \\
24.854 \\
108.42 \\
1322.0 \\
11020 \\
926.41\end{array}$ & $\begin{array}{l}0.89001 \mathrm{E}+06 \\
412.30 \\
19353 \\
0.11190 \mathrm{E}+06 \\
24.286 \\
399.10 \\
1291.8 \\
10768 . \\
905.26\end{array}$ & $\begin{array}{l}0.5562741837 \mathrm{E}+02 \\
0.1028743958 \mathrm{E}-01 \\
0.5458822606 \mathrm{E}+00 \\
0.1110287334 \mathrm{E}+03 \\
0.2022000015 \mathrm{E}-02 \\
0.1020760495 \mathrm{E}-01 \\
0.5314955849 \mathrm{E}-01 \\
0.4683822421 \mathrm{E}+00 \\
0.2823129792 \mathrm{E}-01\end{array}$ \\
\hline
\end{tabular}

clemental composition as strict basis species ....

\begin{tabular}{|c|c|c|c|}
\hline ecles & $m g / 1$ & mg $/ \mathbf{k g}$ & molos/kg \\
\hline $\begin{array}{l}\text { hLo } \\
\text { ca+t } \\
\text { cl- } \\
\text { h+ } \\
\text { hcol- } \\
\text { h+ } \\
\text { mg+t } \\
\text { na+ } \\
\text { so4-. }\end{array}$ & $\begin{array}{l}0.10256 \mathrm{E}+07 \\
421.93 \\
19805 . \\
0.11451 \mathrm{E}+06 \\
126.26 \\
406.42 \\
1322.0 \\
11020 . \\
2775.4\end{array}$ & $\begin{array}{l}0.10021 \mathrm{E}+07 \\
412.30 \\
29353 . \\
0.11190 \mathrm{E}+06 \\
123.38 \\
399.10 \\
1291.8 \\
10768 . \\
2712.0\end{array}$ & 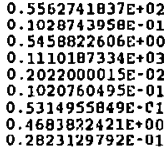 \\
\hline
\end{tabular}

-. equivalent composition of the aqueous phase (cte balances) -..

original basis

species

moles/kg h2o

existing basis

\begin{tabular}{|c|c|c|c|}
\hline 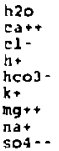 & $\begin{array}{l}0.55627 d 1837 E+02 \\
0.102 B 743958 E-01 \\
0.5458822606 E+00 \\
0.1110187334 E+03 \\
0.2022000015 E-02 \\
0.1020760495 E-01 \\
0.5314955849 E-01 \\
0.4683822421 E+00 \\
0.2823129792 E-01\end{array}$ & 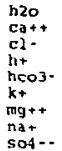 & $\begin{array}{l}0.5562741837 \mathrm{E}+02 \\
0.3028743958 \mathrm{E}+01 \\
0.5458622606 \mathrm{E}+00 \\
0.1110187334 \mathrm{E}+03 \\
0.2022000015 \mathrm{E}-02 \\
0.1020760495 \mathrm{E}-01 \\
0.5314955849 \mathrm{E}-01 \\
0.4683822421 \mathrm{E}+00 \\
0.2823129792 \mathrm{E}-01\end{array}$ \\
\hline
\end{tabular}

single ion activities and activity coefficients are here defined with respect to the rodified nbs ph seale

$\begin{array}{lccc} & \text { ph } & \text { ch } & \text { pe } \\ \text { arodified nbs ph scale } & 8.2200 & 0.5000 & 8.4522 E+00 \\ \text { rational ph scalc } & 8.1079 & 0.5066 & 8.5642 E+00\end{array}$

phel = 8.6852

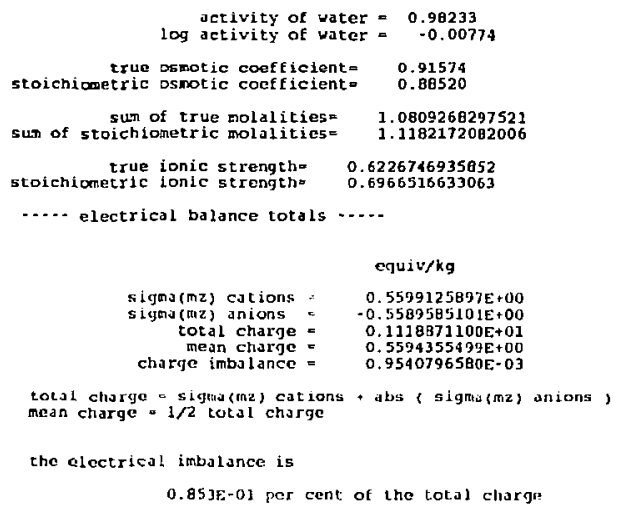


0.170 per cent of che mean charge

0,171 per cent of abs (sigma(taz) anions)

.... activity ratjos of jons .....
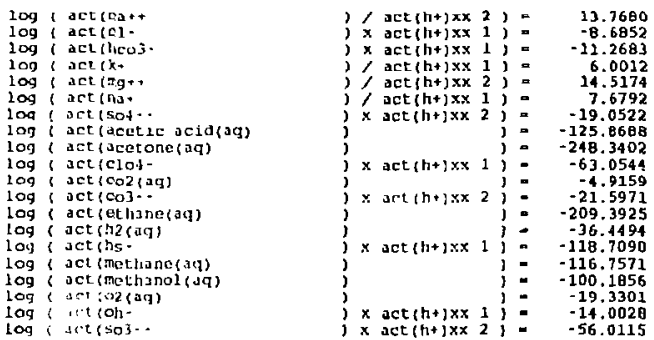

.... distribalacs of aqueous species ....

\begin{tabular}{|c|c|c|c|c|c|}
\hline species & notul cone & $\log \operatorname{con} \mathrm{c}$ & 2099 & activicy & Log act \\
\hline c1. & o. $52-14 E+00$ & -0.2804 & $-0.104 \theta$ & D. $3426 \mathrm{E}+00$ & -0.4652 \\
\hline $\mathrm{na}+$ & 6. $4-1+9 E+00$ & -0.3528 & -0.1801 & $0.2878 E+D O$ & $-0,5408$ \\
\hline $1.19+\%$ & $0.4072 E-01$ & -1.3902 & -0.5325 & $0.1195 E-01$ & $-1,9226$ \\
\hline $\operatorname{sacl}(A q)$ & $0.1648 E-01$ & -1.7830 & 0,0000 & $0.1648 E-01$ & -1.7830 \\
\hline $504-2$ & $0.1322 \mathrm{E}=01$ & -1.0788 & -0.7334 & $0.2442 \mathrm{E}-02$ & 5122 \\
\hline & $0.998] E-02$ & & -0.2100 & $0.6042 E-02$ & -2.2188 \\
\hline $\mathrm{Cu}^{++}$ & $0.9268 \mathrm{E}-02$ & -2.0330 & -0.6390 & $0.2128 E-02$ & -2.6720 \\
\hline $\operatorname{mgsct}(\mathrm{an})$ & $0.753 \mathrm{JE}-02$ & $-2,1232$ & 0.0000 & $0.7531 E=02$ & -2.1232 \\
\hline nasot: & $0.6650 \mathrm{E}-02$ & -2.1772 & -0.2559 & $0.4014 E-02$ & $-2,333 I$ \\
\hline mgc l+ & $0.4638 E-02$ & -2.3336 & -0.1891 & $0.3001 E-02$ & -2.5227 \\
\hline heo3. & $0.1281 E-02$ & -2.8924 & -1.1559 & $0.8946 \mathrm{E}-03$ & -3.0483 \\
\hline casot (aq) & $0.6712 \mathrm{E}-0 \mathrm{~J}$ & -3.1731 & 0,0000 & $0.6712 \mathrm{E}-03$ & $-3,1731$ \\
\hline nahco3 $\left(a a_{1}\right)$ & $0.3672 \mathrm{E}-0 \mathrm{~J}$ & -3.4351 & v.0000 & $0.3672 \mathrm{E}-03$ & -3.4351 \\
\hline $\begin{array}{l}\operatorname{cac} 1+ \\
\text { mghcos+ }\end{array}$ & $0.2271 \mathrm{E}-03$ & $\begin{array}{r}-3.6437 \\
-3.7462\end{array}$ & -0.1891 & $0.1470 \mathrm{E}-03$ & -3.8328 \\
\hline $\begin{array}{l}\text { mghco3+ } \\
\text { K5o4:- }\end{array}$ & $\begin{array}{l}0.1794 E-03 \\
0.1601 E=03\end{array}$ & $\begin{array}{l}-3.7462 \\
-3.7955\end{array}$ & -0.1891 & $0.1161 E+03$ & $\begin{array}{l}-3,9353 \\
-3,9514\end{array}$ \\
\hline mgco3 ( dq) & $\begin{array}{l}0.1601 E-03 \\
0.7927 E-04\end{array}$ & $\begin{array}{l}-3.7955 \\
-4.1009\end{array}$ & $\begin{array}{r}-0.1559 \\
0.0000\end{array}$ & $\begin{array}{l}0.111 E E-03 \\
0.7927 E-04\end{array}$ & $\begin{array}{l}-3.9514 \\
-4.1009\end{array}$ \\
\hline $\mathrm{kcl}(\mathrm{aq})$ & $0.6629 \mathrm{E}-04$ & -9.1786 & 0.0000 & $0.6629 E-04$ & -4.1786 \\
\hline $\operatorname{cac} 12$ (aq) & $0.5676 \mathrm{E}-04$ & -4.2460 & 0.0000 & $0.5676 E-04$ & -4.2160 \\
\hline cahcoj+ & $0.3277 \mathrm{E}-04$ & -4.4846 & -0.1891 & $0.2120 \mathrm{E}-04$ & $-4,6736$ \\
\hline $\operatorname{cacoj}(a q)$ & $0.3147 \mathrm{E}-04$ & -4.5020 & 0.0000 & $0.3147 \mathrm{E}-04$ & -4.5020 \\
\hline cos:- & $0.3068 \mathrm{E}-04$ & -4.5132 & $-0.64 J 9$ & $0.6964 E-0 S$ & $-5,1571$ \\
\hline $\cot (a q)$ & $0.1080 \mathrm{E}-04$ & -4.9667 & 0.0500 & $0.1224 E-04$ & -4.9159 \\
\hline $\begin{array}{l}\text { nacos.- } \\
\text { on- }\end{array}$ & $0.9382 \mathrm{E}-05$ & -5.0277 & -0.1559 & $0.6552 \mathrm{E}-05$ & -5.1836 \\
\hline $\begin{array}{l}\text { on- } \\
\text { nooh(ag) }\end{array}$ & $0.2523 \mathrm{E}-05$ & -5.5980 & -0.1849 & $0.1649 \mathrm{E}-05$ & 28 \\
\hline $\begin{array}{l}\text { nooh (aq) } \\
\text { cjohe }\end{array}$ & $0.3100 \mathrm{E}=06$ & -6.5086 & 0.0000 & $0.3100 E-06$ & $\begin{array}{l}-6.5086 \\
-7.3097\end{array}$ \\
\hline $\begin{array}{l}\text { cuohe } \\
\text { h+ }\end{array}$ & $0.7574 E=07$ & $\begin{array}{l}-7.1207 \\
-8.1079\end{array}$ & $=0.1891$ & $0.4901 \mathrm{E}-07$ & $\begin{array}{l}-7.3097 \\
-8.2200\end{array}$ \\
\hline koh (aq) & $\begin{array}{l}0.7799 E-08 \\
0.3426 E-08\end{array}$ & $\begin{array}{l}-8.1079 \\
-8.4655\end{array}$ & $\begin{array}{r}-0.1121 \\
0.0000\end{array}$ & $\begin{array}{l}0.6026 \mathrm{E}-08 \\
0.3416 \mathrm{E}-08\end{array}$ & $\begin{array}{l}-8.2200 \\
-8.4665\end{array}$ \\
\hline hso4. & $0.2009 \mathrm{E}-06$ & -8.6972 & $=0.1559$ & $0.1402 \mathrm{E}=08$ & -8.0531 \\
\hline hel (aq) & $0.44: \div=09$ & -9.3552 & 0.0000 & $0 .+414 E-09$ & -9.3552 \\
\hline mgt $\operatorname{con} 14$ + & $0.1287 E-11$ & -11.8904 & -2.7012 & o. 256$] \mathrm{E}-14$ & -14. \\
\hline $\begin{array}{l}\text { xhsot (uq) } \\
02(a q)\end{array}$ & $0.5789 E-12$ & -12.2374 & 0.0000 & $0.5769 E-12$ & -12.2374 \\
\hline $\begin{array}{l}02(a q) \\
\text { h2504(ag) }\end{array}$ & $0.4161 E=19$ & -19.3800 & 0.0508 & $0.4677 E-19$ & -19.3301 \\
\hline $\begin{array}{l}\text { h2504(ag) } \\
\text { c10- }\end{array}$ & $\begin{array}{l}0.0468 \mathrm{E}-20 \\
0.8399 \mathrm{E}-25\end{array}$ & $\begin{array}{l}-20.0722 \\
-25.0758\end{array}$ & $\begin{array}{r}0.0000 \\
-0.1559\end{array}$ & $\begin{array}{l}0.8468 E-20 \\
0.5866 E-25\end{array}$ & $\begin{array}{l}722 \\
317\end{array}$ \\
\hline holo(aq) & $0.1309 \mathrm{E}-25$ & -25.8832 & 0.0000 & $0.1309 E-25$ & .25 .8832 \\
\hline ho2- & $0.2519 \mathrm{E}-29$ & $-29.598 \theta$ & -0.1559 & $0.1759 E-29$ & -29.7547 \\
\hline formate & $0.9274 E-36$ & $-35.032 \theta$ & -0.1559 & $0.6476 \mathrm{E}-36$ & -36.1887 \\
\hline$h 2(a q)$ & $0.3161 E+36$ & -36.5001 & 0.0508 & $0.3553 E-36$ & -36.4494 \\
\hline hso 5 - & $0.2356 E-37$ & -37.6279 & -0.1559 & $0.1645 E-37$ & -37.7 \\
\hline so3.- & $0.1+52 \mathrm{E}-38$ & $=78.0381$ & -0.7334 & $0.2682 E-39$ & -39.5715 \\
\hline nsoj- & $0.3713 \mathrm{E}+40$ & -40.4302 & $-0,1559$ & $0.2593 \mathrm{E}-40$ & -40.5861 \\
\hline formic acidsaq) & $0.2209 E-40$ & -40.6558 & 0.0000 & $0.2209 E-40$ & -40.6558 \\
\hline c102- & $0.1789 E-42$ & $-42,7473$ & -0.1559 & $0.1250 \mathrm{E}-42$ & -42.9032 \\
\hline clo3. & $0.2721 \mathrm{E}-46$ & -46.5652 & -0.1559 & $0.1900 \mathrm{E}-46$ & -46.7211 \\
\hline $503(\mathrm{aq})$ & $0.1592 \mathrm{E}-46$ & -46.79 & 0.00 & $0.1592 E-46$ & $-46,7980$ \\
\hline $\operatorname{son}(0 \pi)$ & $0.1153 \vec{b}-40$ & -46.9362 & 0.0000 & $0.1153 E-46$ & $-46,9382$ \\
\hline$n e l e 2(04)$ & $0.111+1-47$ & -47.5532 & 0.0000 & $0.1114 \mathrm{E}-47$ & -47.4312 \\
\hline $5208-$ & $0,8163 \mathrm{E}-53$ & $-53,0082$ & -0.7314 & $0.1508 E-53$ & $-53,8216$ \\
\hline clo4- & $0.2241 E-54$ & -54.6496 & .0 .1846 & $0.1464 E-54$ & $-54,8344$ \\
\hline $5206 \cdots$ & $0.2098 \mathrm{~g}=62$ & -62.6782 & $-0,7334$ & $0.3876 E-63$ & +67.4116 \\
\hline $5205 \cdots$ & a. $x r_{1} 4,3 \mathrm{E}_{1}-0 \mathrm{~S}$ & $-85.2+485$ & -0.7134 & ก. $104 J E-6 G$ & .85 .9819 \\
\hline ncilisnol tayt & $0.6522-100$ & -100.1650 & 0. vo00 & $0.6522-100$ & -100.1856 \\
\hline tis - & $0.4064-110$ & $-110.30+2$ & -0.1848 & $0.3244-110$ & $-110,4890$ \\
\hline $5204 \cdots$ & $0.4126-110$ & -110.3844 & -0.7334 & $0.7624-151$ & -111.1178 \\
\hline$h 25(\Delta q)$ & $0.1900-111$ & .111 .7213 & 0.0000 & $0.2000-111$ & -111.7213 \\
\hline s-. & $0.3183-114$ & $-114 .+707$ & $=0.7334$ & $0.6251-115$ & -115.2041 \\
\hline $5203 \cdots$ & $0.1576-115$ & -115.002 & -0.7334 & $0.2911-116$ & $-116,5359$ \\
\hline mutlane & $0.2 \% 00-110$ & -116.7571 & 0.0000 & $0.1750-116$ & -126.7571 \\
\hline
\end{tabular}




\begin{tabular}{|c|c|c|c|c|c|}
\hline 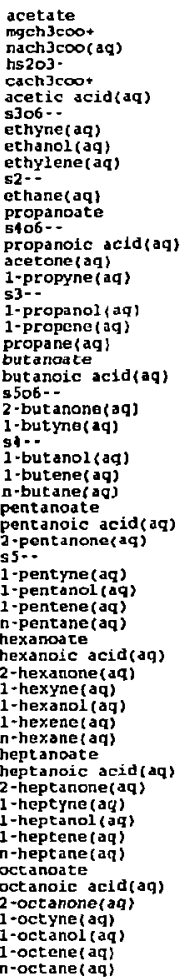 & $\begin{array}{l}0.5623-122 \\
0.1350-122 \\
0.7468-123 \\
0.2595-123 \\
0.1955-123 \\
0.1353-125 \\
0.3869-147 \\
0.2502-183 \\
0.2015-188 \\
0.7020-193 \\
0.2320-196 \\
0.4050-209 \\
0.1592-212 \\
0.5749-716 \\
0.5105-216 \\
0.4569-248 \\
0.7997-270 \\
0.1329-278 \\
0.6533-279 \\
0.3364-281 \\
0.5617-300 \\
0.1402-303 \\
0.4147-307 \\
0.1125+313 \\
0.0000 E+00 \\
0.0000 E+00 \\
0.0000 E+00 \\
0.0000 E+00 \\
0.0000 E+00 \\
0.0000 E+00 \\
0.0000 E+00 \\
0.0000 E+00 \\
0.0000 E+00 \\
0.0000 E+00 \\
0.0000 E+00 \\
0.0000 E+00 \\
0.0000 E+00 \\
0.0000 E+00 \\
0.0000 E+00 \\
0.0000 E+00 \\
0.0000 E+00 \\
0.0000 E+00 \\
0.0000 E+00 \\
0.0000 E+00 \\
0.0000 E+00 \\
0.0000 E+00 \\
0.0000 E+00 \\
0.0000 E+00 \\
0.0000 E+00 \\
0.0000 E+00 \\
0.0000 E+00 \\
0.0000 E+00 \\
0.0000 E+00 \\
0.0000 E+00 \\
0.0000 E+00 \\
0.0000 E+00 \\
0.0000 E+00 \\
0.0000 E+00 \\
0.0000 E+00\end{array}$ & $\begin{array}{l}-122.2500 \\
-122.8695 \\
-123.1268 \\
-123.5959 \\
-123.7089 \\
-125.8688 \\
-147.4124 \\
-183.5890 \\
-189.6957 \\
-193.1537 \\
-196.6346 \\
-209.3925 \\
-212.7979 \\
-216.2404 \\
-216.2920 \\
-248.3402 \\
-270.0971 \\
-278.8766 \\
-279.1849 \\
-281.4731 \\
-300.2505 \\
-303.8295 \\
-307.3822 \\
-313.9468 \\
-339.0859 \\
-361.1213 \\
-361.3385 \\
-370.8836 \\
-372.6808 \\
-391.1640 \\
-394.7512 \\
-396.2820 \\
-430.2495 \\
-444.0203 \\
-452.1309 \\
-460.6032 \\
-463.7123 \\
-482.1474 \\
-485.7755 \\
-489.2916 \\
-521.0979 \\
-543.2359 \\
-552.2359 \\
-554.5535 \\
-573.2742 \\
-736.6386 \\
-755.2202\end{array}$ & $\begin{array}{r}-0.1559 \\
-0.1891 \\
0.0000 \\
-0.1559 \\
-0.1891 \\
0.0000 \\
-0.7334 \\
0.0000 \\
0.0000 \\
0.0000 \\
-0.7334 \\
0.0000 \\
-0.1559 \\
-0.7334 \\
0.0000 \\
0.0000 \\
0.0000 \\
-0.7334 \\
0.0000 \\
0.0000 \\
0.0000 \\
-0.1559 \\
0.0000 \\
-0.7334 \\
0.0000 \\
0.0000 \\
-0.7334 \\
0.0000 \\
0.0000 \\
0.0000 \\
-0.1559 \\
0.0000 \\
0.0000 \\
-0.7334 \\
0.0000 \\
0.0000 \\
0.0000 \\
0.0000 \\
-0.1559 \\
0.0000 \\
0.0000 \\
0.0000 \\
0.0000 \\
0.0000 \\
0.0000 \\
-0.1559 \\
0.0000 \\
0.0000 \\
0.0000 \\
0.0000 \\
0.0000 \\
0.0000 \\
-0.1559 \\
0.0000 \\
0.0000 \\
0.0000 \\
0.0000 \\
0.0000 \\
0.0000\end{array}$ & $\begin{array}{l}0.3927-122 \\
0.073 \mathrm{-123} \\
0.7468-123 \\
0.1812-123 \\
0.1265-123 \\
0.1353-125 \\
0.7148-148 \\
0.2582-183 \\
0.2015-188 \\
0.7020-193 \\
0.4286-197 \\
0.4050-209 \\
0.1112-212 \\
0.1062-216 \\
0.5105-216 \\
0.4569+248 \\
0.7997-270 \\
0.2455-279 \\
0.6533-279 \\
0.3364-281 \\
0.5617-300 \\
0.1034-303 \\
0.4147-307 \\
0.2078-314 \\
0.0000 E+00 \\
0.0000 E+00 \\
0.0000 E+00 \\
0.0000 E+00 \\
0.0000 E+00 \\
0.0000 E+00 \\
0.0000 E+00 \\
0.0900 E+00 \\
0.0000 E+00 \\
0.0000 E+00 \\
0.0000 E+00 \\
0.0000 E+00 \\
0.0000 E+00 \\
0.0000 E+00 \\
0.0000 E+00 \\
0.0000 E+00 \\
0.0000 E+00 \\
0.0000 E+00 \\
0.0000 E+00 \\
0.0000 E+00 \\
0.0000 E+00 \\
0.0000 E+00 \\
0.0000 E+00 \\
0.0000 E+00 \\
0.0000 E+00 \\
0.0000 E+00 \\
0.0000 E+00 \\
0.0000 E+00 \\
0.0000 E+00 \\
0.0000 E+00 \\
0.0000 E+00 \\
0.0000 E+00 \\
0.0000 E+00 \\
0.0000 E+00 \\
0.0000 E+00\end{array}$ & 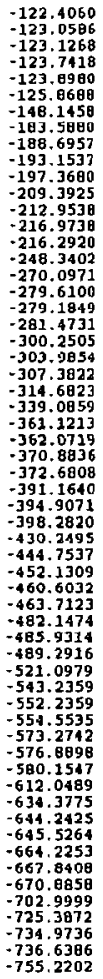 \\
\hline
\end{tabular}

\footnotetext{
.... major aqueous species contributing to mass balances ....
}

aqueous species accounting for 998 or more of ca+t

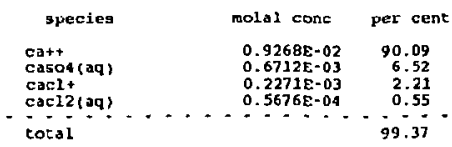

aqueous species accounting for 991 or store of el-

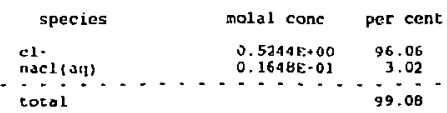

aquerous species Jccounting for 491 or more of hco3-

species

hcos.

nahcoj (dq)

mighcoj+ solal cone

$0.12 B 1 E-02$ $0.3672 \mathrm{E}-03$ per cent

63.36

18.16
8.67 


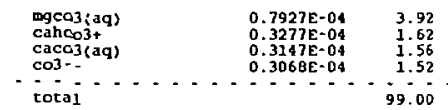

aqueous species accounting for 996 or more of $\mathrm{x}+$

\begin{tabular}{lcc} 
species & molal conc & per cent \\
k+ & $0.9981 \mathrm{E}-02$ & 97.78 \\
kso4- & $0.1601 \mathrm{E}-03$ & 1.57 \\
\hline $\operatorname{totaj}$ & $-\ldots .35$
\end{tabular}

aqueous species accounting for 991 or more of $\mathrm{mg}++$

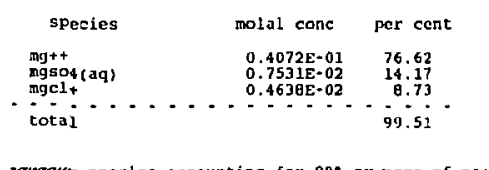

squerests sperter decoumting for gst or more of ndt

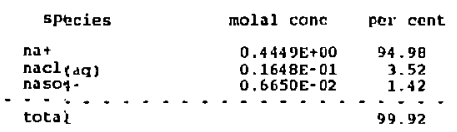

aqueouts species accounting for 991 or moro of so4--

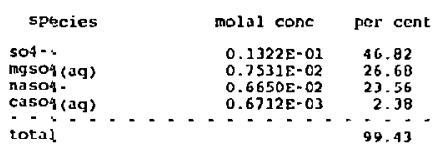

... summary of aqueous redox reactions .....

\begin{tabular}{|c|c|c|c|c|}
\hline Couple & ch, volts & pe: & $\log 102$ & $a h, k 001$ \\
\hline 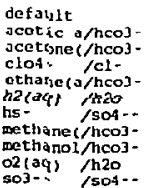 & $\begin{array}{l}0.500 \\
0.500 \\
0.500 \\
0.500 \\
0.500 \\
0.500 \\
0.500 \\
0.500 \\
0.500 \\
0.500 \\
0.500\end{array}$ & $\begin{array}{l}0.8452 E+01 \\
0.8452 E+01 \\
0.8452 E+01 \\
0.8452 E+01 \\
0.0452 E+01 \\
0.8452 E+01 \\
0.8452 E+01 \\
0.8452 E+01 \\
0.8452 E+01 \\
0.8452 E+01 \\
0.8452 E+01\end{array}$ & $\begin{array}{l}-16.432 \\
-16.432 \\
-16.432 \\
-16.432 \\
-16.432 \\
-14.432 \\
-16.432 \\
-16.432 \\
-16.432 \\
-16.432 \\
-16.432\end{array}$ & $\begin{array}{l}11.531 \\
11.531 \\
11.531 \\
11.531 \\
11.531 \\
21.531 \\
11.531 \\
11.531 \\
11.531 \\
11.531 \\
11.531\end{array}$ \\
\hline
\end{tabular}

-... summary of aqueous non-equilibrium non-redox redctions -...

couple affinity, keal

none

.... sumary of stoichiometric mincral saturacion states ....

(minerals with affinicies. 15 . - 10 kcol are not listed)

\begin{tabular}{|c|c|c|c|c|c|c|c|}
\hline mincral & $\mathrm{loq} \pi / k$ & act, kcal & state & tneral & $\log q / k$ & aft, $x \operatorname{cal} l$ & state \\
\hline $\begin{array}{l}\text { onhydrite } \\
\text { arcasite } \\
\text { bassantee } \\
\text { bloedite } \\
\text { calcite } \\
\text { dolomite } \\
\text { dolomite-ord } \\
\text { gaylussice } \\
\text { gypsum }\end{array}$ & $\begin{array}{r}-0.978 \\
-5.271 \\
-1.021 \\
-5.762 \\
0.051 \\
3.235 \\
3.235 \\
-5.613 \\
-0.817\end{array}$ & $\begin{array}{r}-1.334 \\
-7.161 \\
-2.213 \\
-7.898 \\
0.808 \\
7.414 \\
4.414 \\
-6.294 \\
-1.115\end{array}$ & $\begin{array}{l}\text { ssjed } \\
\text { ssatd } \\
\text { ssatil }\end{array}$ & $\begin{array}{l}\text { Jragonite } \\
\text { arcinice } \\
\text { bischofite } \\
\text { brucite } \\
\text { caso4:0.5hzo(beta) } \\
\text { dolomite-dis } \\
\text { epsomite } \\
\text { glauberice } \\
\text { malite }\end{array}$ & $\begin{array}{r}0.507 \\
-1.920 \\
-7.2 \times 2 \\
-1.796 \\
-1.795 \\
1.691 \\
-2.627 \\
=3.509 \\
-2.592\end{array}$ & $\begin{array}{r}0.691 \\
-2.611 \\
-9.948 \\
-2.450 \\
-2.448 \\
2.307 \\
-3.584 \\
-4.797 \\
-3.536\end{array}$ & ssatd \\
\hline
\end{tabular}




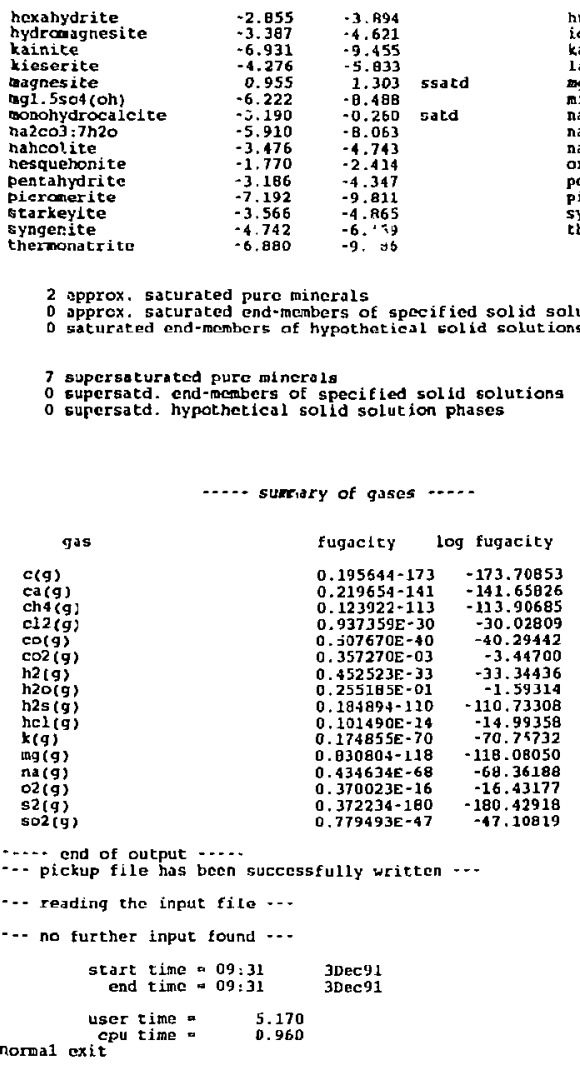

\subsection{The Sea Water Test Case, Using Pitzer's Equations}

The preceding test case can also be run using Pitzer's equations. The results presented here were obtained using the $\mathbf{h m w}$ data file, which is based on Harvie, Moller, and Weare (1984). The only functional difference between the input file used here and that in the previous case is that iopg1 $=1$ in the present case. The input files are shown in both " $W$ " and " $D$ " formats. The output file is reproduced here beginning with the message announcing the end of Newton-Raphson iteration.

The results differ from those in the previous section because the underlying models are different. In facl, some guantities, including individual species molalities and activity coefficients, can not be compared in a meaningful way. This is due to the fact that the models utilize different sets of species to represent the systems. Note that far fewer species appear in the present case. The only 
ion pairs represented are $\mathrm{CaCO}_{3(a q)}, \mathrm{MgCO}_{3(a q)}$, and $\mathrm{MgOH}^{+}$. Also note that no organics appear on the present output file, as there are none in this model.

Some parameters which can be compared are the activities and activity coefficients of the electrically neutral species common to both models (mostly basis species). For the ions, electrically neutral combinations of these parameters can also be compared. The single-ion activities and activity coefficients themselves can be compared, but not in a truly exact sense. Note that in both the present case and the previous one, the iopgl option switch is set to 0 , causing all single-ion activities and activity coefficients to be normalized to the NBS pH scale. However, the exact definition of this scale in a solution as concentrated as sea water has some ionic strength dependence, and the ionic strength of this water is slightly different in the two models $(0.6964$ versus 0.6227 molal). The saturaticil indices of the various minerals common to both models can be compared with no problem. In the present case, the $S I$ for calcite is +0.645 ; in the previous case, it was +0.651 . This is reasonably good agreement, though the results in the present case are without doubt more acurate.

The EQ3NR input file (swmain.3i), the sea water benchmark test case using Pitzer's equations ("W" format):

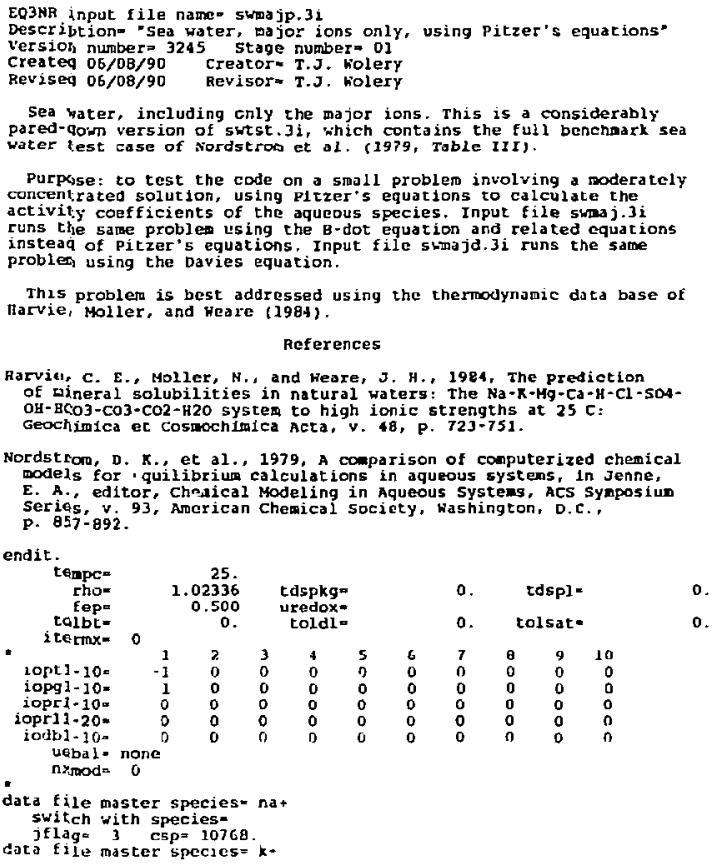




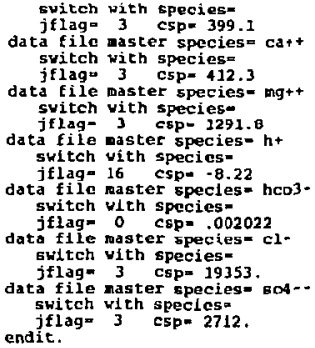

The EO3NR input file (swmain.3i), sea water benchmark test vase using Pitzer's equations ("D" format):

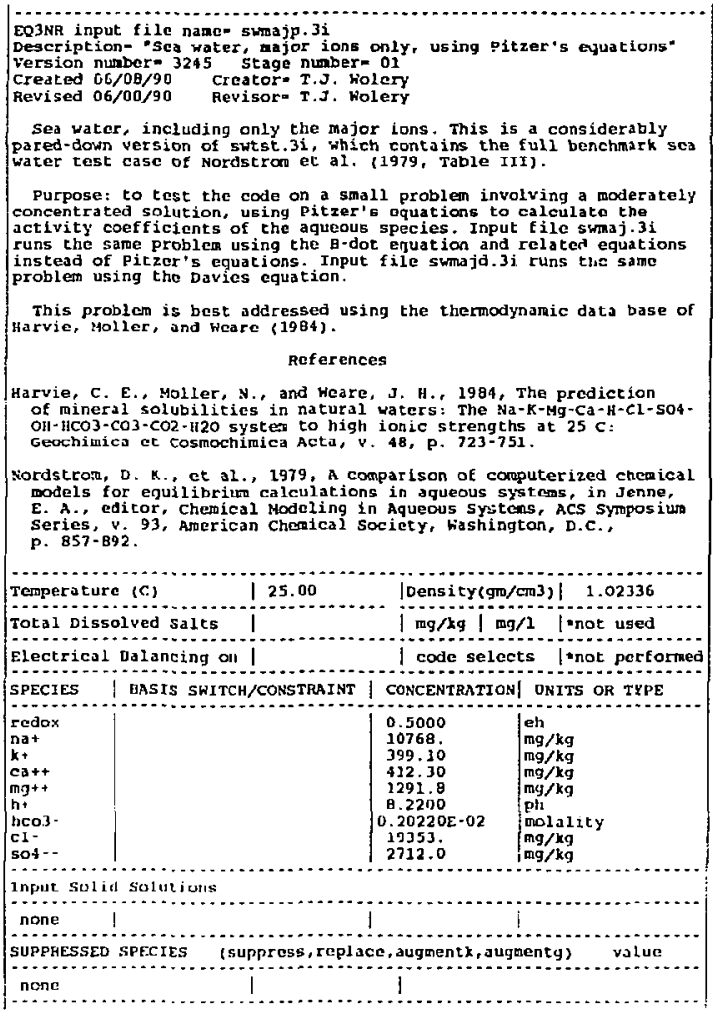




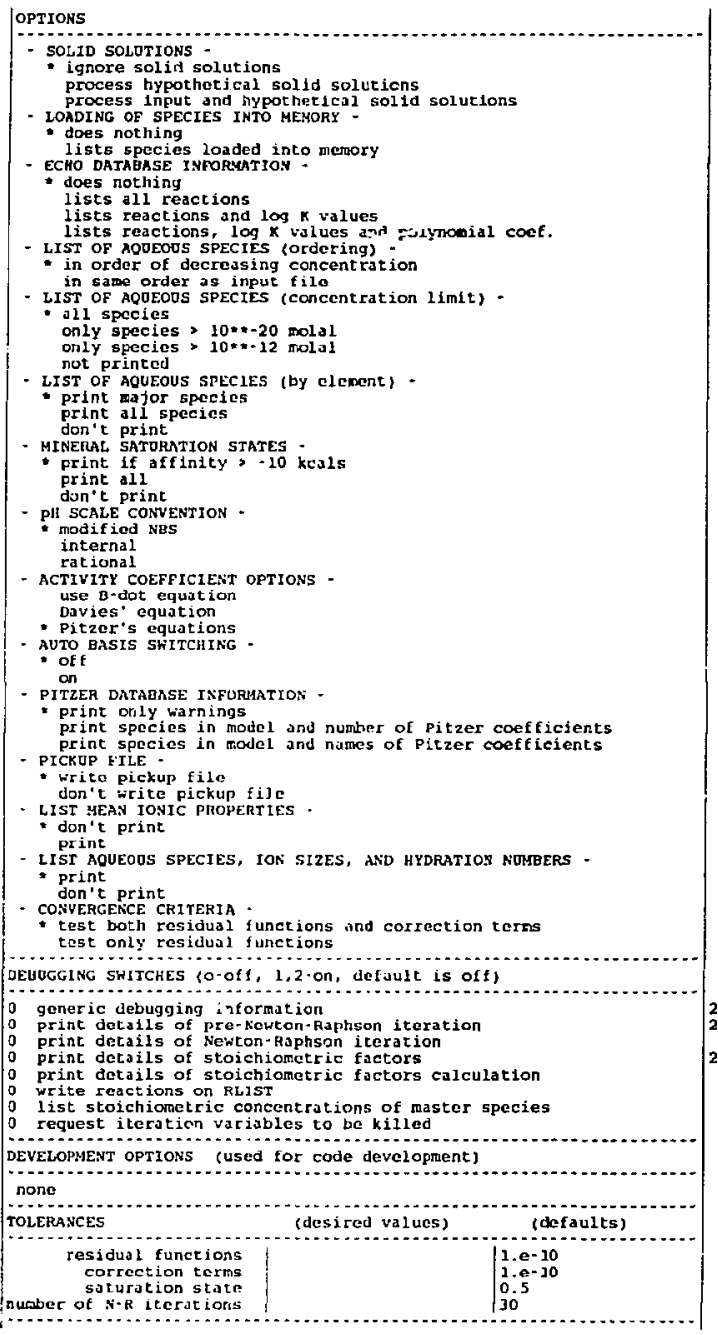


The EQ3NR outout file (swmain.30), the sea water benchmark test case using Pitzer's equations (beginning with the message announcing the end of Newton-Raphson iteration):

(Material delefed)

...- Elemental composition of the aquoous phase .....

\begin{tabular}{|c|c|c|c|}
\hline Lement & $09 / 1$ & $\mathrm{mg} / \mathrm{kg}$ & moles/kg \\
\hline $\begin{array}{l}\text { D } \\
\text { ca } \\
\mathrm{cL} \\
\mathrm{h} \\
\mathrm{c} \\
\mathrm{k} \\
\mathrm{mg} \\
\mathrm{na} \\
\mathrm{s}\end{array}$ & $\begin{array}{l}0.210805+06 \\
421.93 \\
19805 \\
0.114512+06 \\
24.054 \\
408.42 \\
1322.0 \\
11020 . \\
926.41\end{array}$ & $\begin{array}{l}0.890012+06 \\
412.30 \\
19353 \\
0.11190 \mathrm{z}+06 \\
24.286 \\
399.10 \\
1291.8 \\
10768 . \\
905.26\end{array}$ & $\begin{array}{l}0.5562742039 E+02 \\
0.1028743949 E-01 \\
0.5458822510 E+00 \\
0.1110145924 k+03 \\
0.2 \\
0.1020760493 E-01 \\
0.531495770 E-01 \\
0.4683822413 E+00 \\
0.2823129677 E-01\end{array}$ \\
\hline
\end{tabular}

...- elemental composition as strict basis species .....

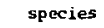

\begin{tabular}{|c|c|}
\hline $\begin{array}{l}\text { h2o } \\
\text { cat+ } \\
c 1+ \\
h+ \\
\text { heos- } \\
k+ \\
\text { ng+t } \\
\text { nat } \\
\text { sot-. }\end{array}$ & $\begin{array}{l}0.10256 \mathrm{E}+07 \\
421.92 \\
19805 \\
0.12451 \mathrm{E}+06 \\
126.26 \\
408.42 \\
1322.0 \\
11020 . \\
2775.4\end{array}$ \\
\hline
\end{tabular}

$$
\mathbf{n g} \mathbf{k g}
$$

$0.10001 E+07$

412.30
19353.

$0.11190 \mathrm{E}+06$

123.39

399.10

3291.8

10768. moles/kg

$0.5562742039 \mathrm{E}+02$

$0.1028743949 E-01$

$0.5458022600 E+00$

$0.1110186924 E+03$

0.2022000000E-02

0.1020760493E-01

$0.5314955770 \mathrm{E}-01$

$0 .\{683822413 E+00$
$0.2823129677 E-01$

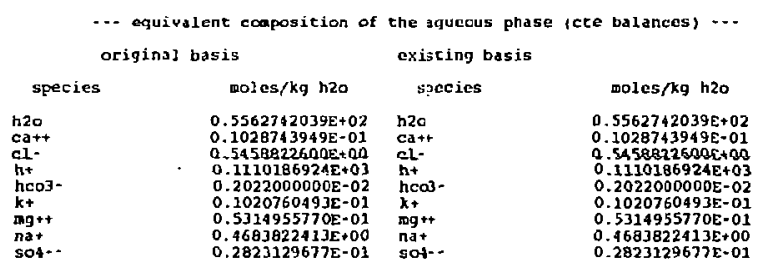

single ion activities and activity coefficients are here defined vith respect to the modified nbs ph scale

$\begin{array}{lccc} & \text { ph } & \text { el } & \text { Po } \\ \text { modified nbs ph scale } & 8.2200 & 0.5000 & 8.4522 E+00 \\ \text { rational ph scole } & 8.1132 & 0.5053 & \text { B.5590E+00 }\end{array}$

phel $=8.6722$

$$
\begin{aligned}
\text { activity of uate }= & 0.98198 \\
\text { loy activity of nute: }= & -0.00790
\end{aligned}
$$

true osmotic coefficient $=0.90273$

stoichionatric osmotic coefficient $=0.90255$

sum of true molalities- 1.1180579526987

sum of stoichiometric molalitiese 1.1182763340013

true lonic strength $=0.6964346415728$ 


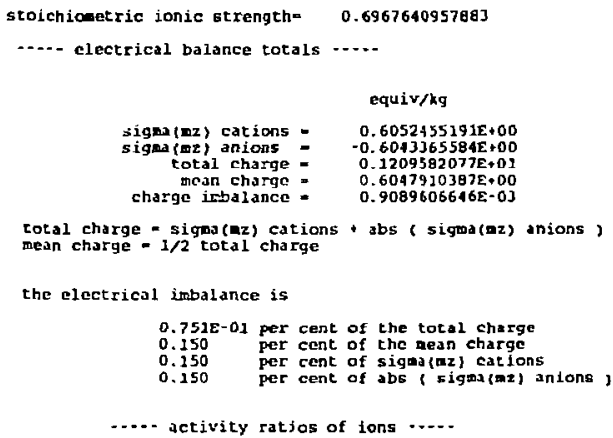

the electricol imbalance is

$0.751 E-01$ per cent of the total charge

0.150 per cent of the nean charge

0.150 per cent of sigaa \{mz\} cations

0,150 per cent of abs (figma(mz) anions,

...- activity ratjos of lons ....

log (acticat.

$\log$ actict.

$\log$ (actict:-

$\log (\operatorname{act}(h \mathrm{cos})$

$\log$ ( act $(x+$

109 i act $(\mathrm{mg}+$

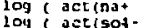

200 (act (coz)

$\log \{$ act $(\cos -$

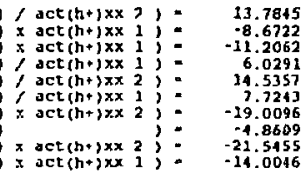

.... distribution of aqueous species ....

\begin{tabular}{|c|c|c|c|c|c|}
\hline species & malal cone & $\log$ canc & $\log 9$ & activity & $\log$ aet \\
\hline 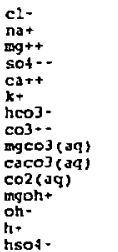 & $\begin{array}{l}0.5459 E+00 \\
0.4694 E+00 \\
0.5306 E-01 \\
0.282 J E-01 \\
0.1026 E-01 \\
0.1021 E-01 \\
0.1816 E-02 \\
0.8641 E-04 \\
0.8292 E-04 \\
0.2456 E-04 \\
0.1224 E-04 \\
0.3362 E-05 \\
0.3029 E-05 \\
0.7706 E-08 \\
0.2332 E-08\end{array}$ & $\begin{array}{l}-0.2629 \\
-0.3294 \\
-1.2752 \\
-1.5493 \\
-1.9887 \\
-3.9911 \\
-2.7409 \\
-4.0635 \\
-4.0313 \\
-4.6097 \\
-4.9121 \\
-5.4735 \\
-5.5168 \\
-6.1132 \\
-6.6322\end{array}$ & $\begin{array}{r}-0.1893 \\
-0.1653 \\
-0.6291 \\
-1.0204 \\
-0.6668 \\
-0.1998 \\
-0.2452 \\
-1.0420 \\
0.0000 \\
0.0000 \\
0.0512 \\
-0.0279 \\
-0.2658 \\
-0.1068 \\
-0.1788\end{array}$ & $\begin{array}{l}0.3530 E+00 \\
0.3194 E+00 \\
0.1247 E-01 \\
0.2694 E-02 \\
0.2210 E-02 \\
0.6444 E-02 \\
0.1032 E-02 \\
0.7644 E-05 \\
0.8292 E-04 \\
0.2456 E-04 \\
0.1378 E-04 \\
0.3152 E-05 \\
0.1642 E-05 \\
0.6026 E-08 \\
0.1545 E-08\end{array}$ & $\begin{array}{l}-0.4522 \\
-0.4957 \\
-1.9043 \\
-2.5696 \\
-2.6555 \\
-2.1909 \\
-2.9862 \\
-5.1055 \\
-4.0813 \\
-4.6097 \\
-4.8609 \\
-5.5014 \\
-5.7846 \\
-8.2200 \\
-8.8110\end{array}$ \\
\hline
\end{tabular}

.... mujor aqueous species contributing to mass balances .....

agueous species accounting for 991 or more of ca+t

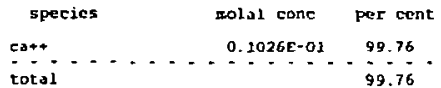

aqueous species accounting for $99 \mathrm{t}$ or more of $\mathrm{cl}$ -

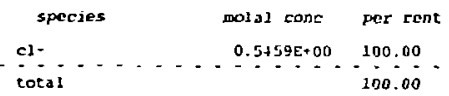

aqueaus species aecounting for jov or tiofe of heos-

species molal cote per cent.

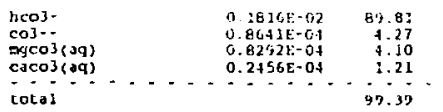


equeous species accounting for 998 or core of $\mathrm{kt}$

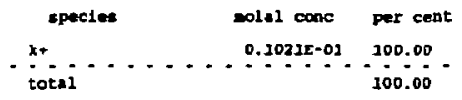

uquecus species accounting for 991 or more of $\mathrm{wg}++$

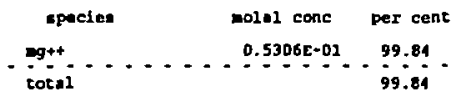

equeous spectes ecounting for 998 or more of nat

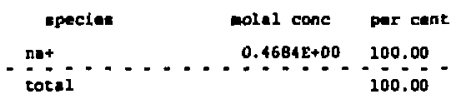

aquerus spetes accounting for 994 or wore of tor--

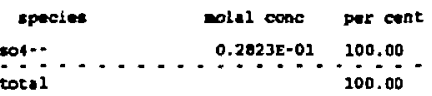

-... dunary of aqueous redox reactions -...-

couple eh, volts pe- $\log$ fo2 ah, kcal

$\begin{array}{lllll}\text { defaule } & 0.500 & 0.8452 E+01 & -16.432 & 11.531\end{array}$

.... subary of aqueous non-equilibriu non-redox reactions .....

couple affintty, kal

none

.... turery of stolchicaetric sineral saturation states -...

(ainerale vith affinicies .1t. - I0 keal are not 11sted)

\begin{tabular}{|c|c|c|c|c|c|c|c|}
\hline nineral & $\log g / k$ & aff, teal & Etate & nineral & $\log q / k$ & eff, kad & state \\
\hline 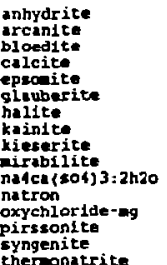 & $\begin{array}{l}-0.863 \\
-5.175 \\
-5.720 \\
0.645 \\
-2.648 \\
-3.542 \\
-2.519 \\
-6.948 \\
-1.359 \\
-2.412 \\
-6.691 \\
-5.351 \\
-5.686 \\
-4.630 \\
-4.736 \\
-6.587\end{array}$ & $\begin{array}{r}-1.177 \\
-7.060 \\
-7.803 \\
0.080 \\
-3.613 \\
-4.032 \\
-3.436 \\
-9.479 \\
-5.947 \\
-3.291 \\
-9.128 \\
-7.300 \\
-7.757 \\
-6.317 \\
-6.461 \\
-6.986\end{array}$ & stat & 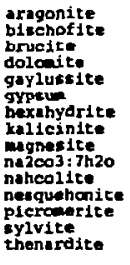 & $\begin{array}{r}0.458 \\
-7.311 \\
-2.589 \\
2.312 \\
-4.476 \\
-0.560 \\
-2.886 \\
-5.458 \\
0.824 \\
-5.692 \\
-3.079 \\
-1.866 \\
-7.145 \\
-3.543 \\
-3.274\end{array}$ & $\begin{array}{r}0.626 \\
-9.975 \\
-3.532 \\
3.154 \\
-6.107 \\
-0.901 \\
-3.938 \\
-7.447 \\
1.124 \\
-7.765 \\
-4.200 \\
-2.546 \\
-9.748 \\
-4.834 \\
-4.466\end{array}$ & $\begin{array}{l}\text { osatd } \\
\text { anatd }\end{array}$ \\
\hline
\end{tabular}

- approx. seturated pure minerals

0 approx. Saturated end-reabers of sperified solid solutions

o saturated end-nembers of hypothetical eolin solutions

4 superseturated pure minerals

0 supersetd. ent-mbers of spectifted colid soluctons

o oupereatd. hypothetical oolid colution phases 


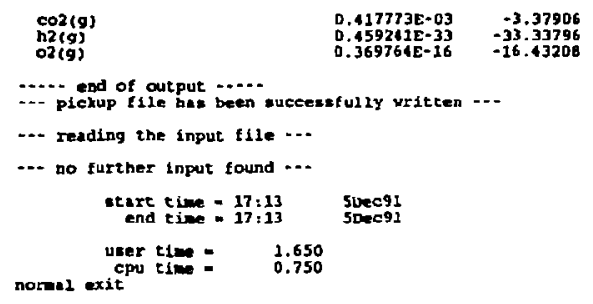

\subsection{Using Mineral Solubility Constraints: An Example}

This test case is taken from INTERA (1983), which used it to compare EQ3/6 with PHREEQE (Parkhurst, Plummer, and Thorstenson, 1980). In it, dissolved calcium is constrained to satisfy equilibrium with calcite and dissolved iron is constrained to satisfy equilibrium with hematite. The bicarbonate is constrained to satisfy an equilibrium $\mathrm{CO}_{2}$ fugacity of $10^{-2}$ bar. On top of that. the $\mathrm{pH}$ is adjusted to satisfy electrical balance. The input files are presented here in both formats. The output file is presented beginning with the message announcing the end of Newton-Raphson iteration. The results shown were obtained using the com data file. The activity coefficients were computed from the B-dot equation. Note the appearance of the organic species, which all have negligible concentrations at the relatively high oxygen fugacity.

Note that the calculated saturation indices for calcite and hematite written on the output file are indeed zero. Similarly, the calculated equilibrium $\mathrm{CO}_{2}$ fugacity is $10^{-2}$ bar. The $\mathrm{pH}$ adjustment is relatively minor. In problems such as this, if the input $p H$ value is too far off the mark (usually more than 1-3 pH units), convergence problems are likely to occur. More than that, problems using some of the kind of constraints used here, especially in combination, may be ill-defined and have no realistic solution.

The EO3NR input file (oxcalhem 3i). mineral solubility equilibrium test case ("W" format):

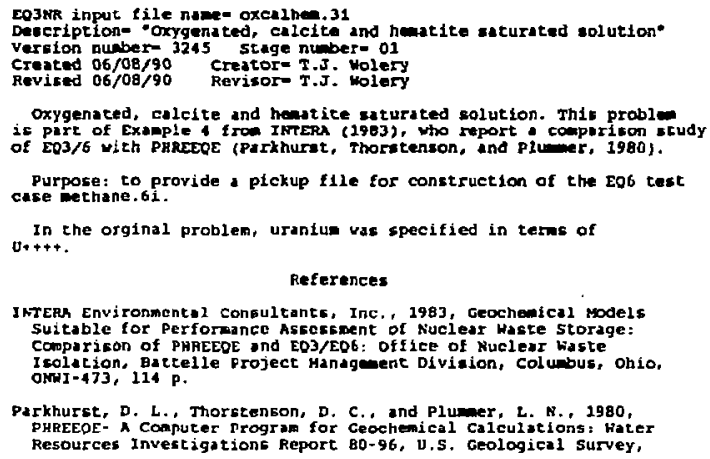


Reston, virginia, $210 \mathrm{p}$.

endit.

rho

Eep=

tolbt

iterm $x=$

iopt $1-10=$

iopg $1-10$ *

iopr $1-10$

iop $11-20=$

1OPI $11-20$

$\alpha i b 1-10=$

uebal $=n+$
nxmod $=$

data file master species* nat

switch with speciess

data file muster species $\approx \mathrm{Ca}+$ fwitch with species"

jflag= 19 csp= 0 .

- culcite

data file master species fet Gwitch with species= jflag= 19 cspa 0.

mineral- homatite

data file master species" uo2,t switch vith species.

data file moster species heo3. switch with species.

jflug=21 csp=-2,0

mineral- $\operatorname{coz}(g)$

data file master species" soa-5witch with spccies"

data file master species- clswitch with species= jflagm 0 csp= 5.0e-3

data file master species- $h$ t switch with species=

jf $l a g=16 \quad \operatorname{csp}=-7.40$

The EQ3NR input file (oxcalhem.3i), mineral solubility equilibrium test case ("D" format):

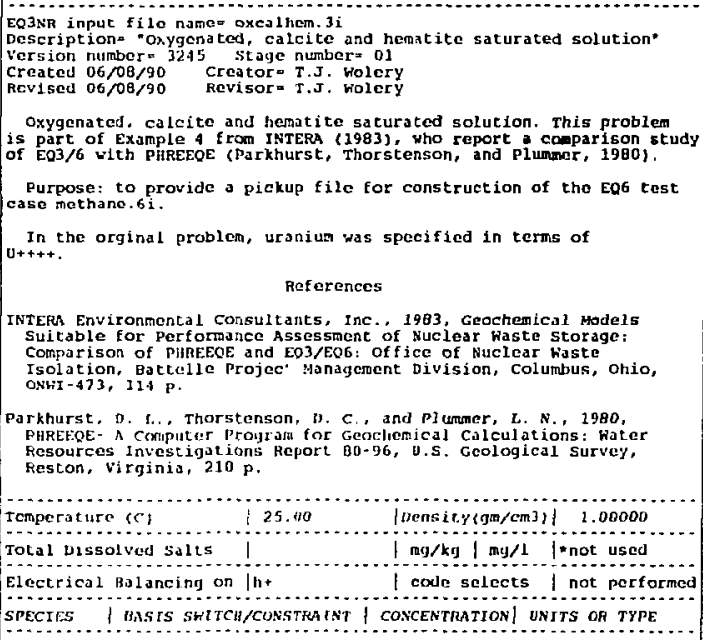




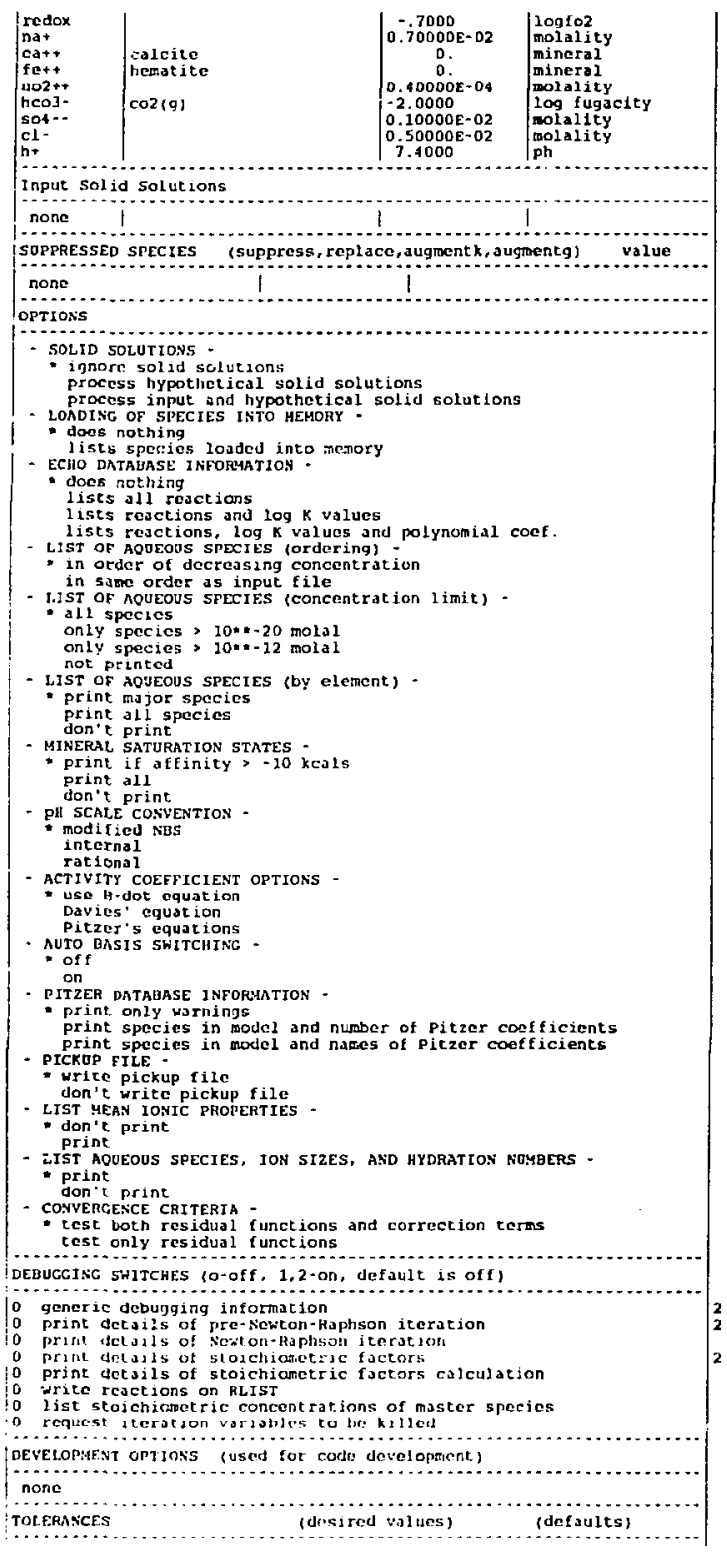




\begin{tabular}{|c|c|}
\hline $\begin{array}{r}\text { residual functions } \\
\text { correction terms } \\
\text { gaturation state } \\
\text { number of } \mathrm{N}-\mathrm{R} \text { iterations }\end{array}$ & $\mid \begin{array}{l}1 . e-10 \\
1 . e-10 \\
0.5 \\
30\end{array}$ \\
\hline
\end{tabular}

The EQ3NR output file (oxcalhem, 3 ), mineral solubility equilibrium test case (beginning with the message announcing the end of Newton-Raphson iteration):

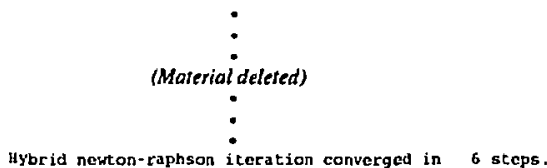

....- Surmary of the aqueous phase .....

.... Elemental composition of the aqueous phase ....

$\begin{array}{cccc}\text { clenent } & \text { mg/l } & \text { mg/kg } & \text { moles } / \mathrm{kg} \\ \text { o } & 0.88836 E+05 & \mathbf{0 . 8 8 8 3 6 E + 0 6} & 0.5552474398 E+02 \\ \text { ca } & \mathbf{7 4 . 2 4 8} & 74.248 & 0.1852584728 E-02 \\ \text { cl } & 177.26 & 377.26 & 0.500000000 E-02 \\ \text { fe } & 0.50317 E-07 & 0.50317 E-07 & 0.900975098 B E-12 \\ \text { h } & 0.11100 E+06 & 0.11190 E+06 & 0.1110204565 E+03 \\ \text { c } & \$ 1.284 & 48.284 & 0.4019986372 E-02 \\ \text { na } & 160.93 & 160.93 & 0.7000000000 E-02 \\ \text { s } & 32.066 & 32.066 & 0.1000000001 E-02 \\ \text { u } & 9.5212 & 9.5212 & 0.399999998 E-04\end{array}$

.... elemental composition as strict busis species .....

\begin{tabular}{|c|c|c|c|}
\hline species & $m g / 1$ & $\mathrm{mg} / \mathrm{kg}$ & moles/kg \\
\hline$+t$ & $\begin{array}{l}0.10003 E+07 \\
74.246 \\
177.26 \\
0.50317 E-07 \\
0.11190 \mathrm{E}+06 \\
245.29 \\
160.93 \\
96.064 \\
10.801\end{array}$ & $\begin{array}{l}0.10003 E+07 \\
74.248 \\
177.26 \\
0.50317 E-07 \\
0.11190 E+06 \\
245.29 \\
160.93 \\
96.064 \\
10.801\end{array}$ & $\begin{array}{l}0.555247439 \mathrm{EE}+02 \\
0.1852584728 E-02 \\
0.5000000000 E-02 \\
0.9009750988 E-12 \\
0.1110204565 E+03 \\
0.4019986372 E-02 \\
0.7000000000 E-02 \\
0.1000000001 E-02 \\
0.3999999985 E-04\end{array}$ \\
\hline
\end{tabular}

-. equivalent composition of the aqueous phase (ete balanees) -..

original basis

existing basis

species moles/kg h2o species moles/kg hzo

\begin{tabular}{|c|c|c|c|}
\hline 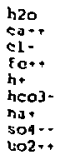 & $\begin{array}{l}0.555247439 \mathrm{BE}+02 \\
0.185258472 \mathrm{EE}-02 \\
0.5000000000 \mathrm{0}+02 \\
0.900975098 \mathrm{BE}-12 \\
0.1110204565 \mathrm{0}+03 \\
0.4019986372 \mathrm{E}-02 \\
0.7000000000 \mathrm{0}-02 \\
0.1090000001 \mathrm{E}-02 \\
0.399909995 \mathrm{E}-04\end{array}$ & $\begin{array}{l}\text { h2o } \\
\mathrm{cat+} \\
\mathrm{cl}- \\
\mathrm{fe+t} \\
\mathrm{h}+ \\
\text { heo3- } \\
\text { not } \\
\text { so4-- } \\
\text { vo2++ }\end{array}$ & $\begin{array}{l}0.555247439 \mathrm{BE}+02 \\
0.1652584728 \mathrm{E}-02 \\
0.5000000000 \mathrm{E}-02 \\
0.9009750988 \mathrm{E}-12 \\
0.1110204565 \mathrm{E}+03 \\
0.4019996372 \mathrm{E}-02 \\
0.7000000000 \mathrm{E}-02 \\
0.300000000 \mathrm{E}-02 \\
0.3999999985 \mathrm{E}-04\end{array}$ \\
\hline
\end{tabular}

single ton activities and sct.tuity coefficionts are bere defunet with respect to the modified nbs ph sedie

modified nos ph scole

rational ph scalo

Plicl $=9.6624$ th

nll

7.2655
0.7852
$0.7 \mathrm{BBg}$ me

1. $3290 \mathrm{D}+02$ 1. 33365.01 


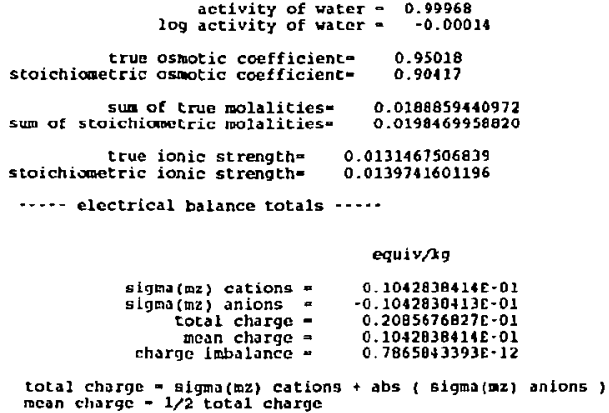

$\begin{array}{ll}\text { input } & -7.4000 \\ \text { Einal } & -7.3108 \\ \text { jdj } & 0.89175 E-01\end{array}$

.... activity ratios of ions -...
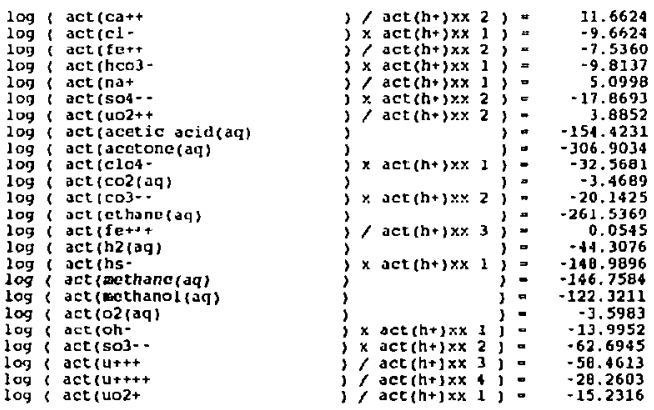

.... distribucion of aqueous specics ....

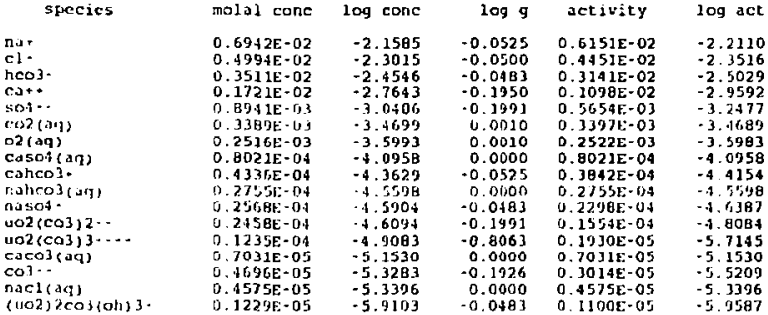




\begin{tabular}{|c|c|c|c|c|c|}
\hline 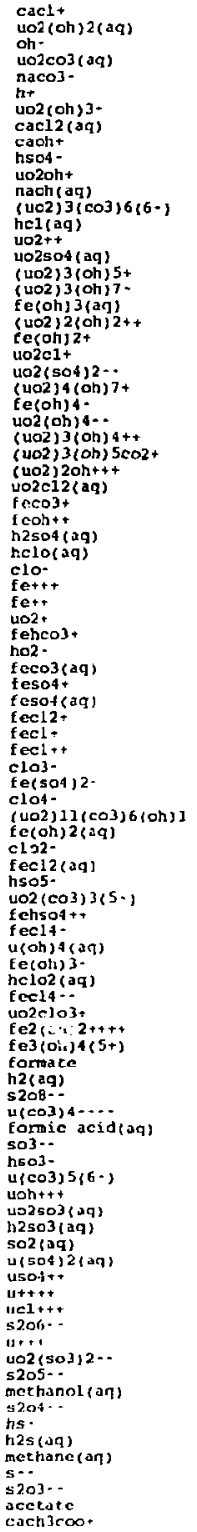 & 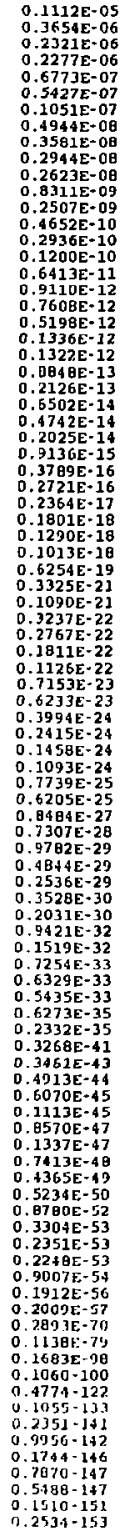 & 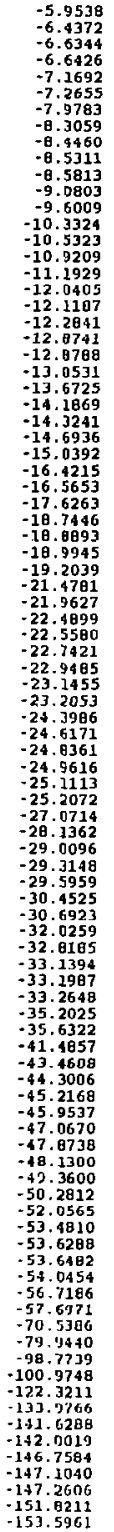 & 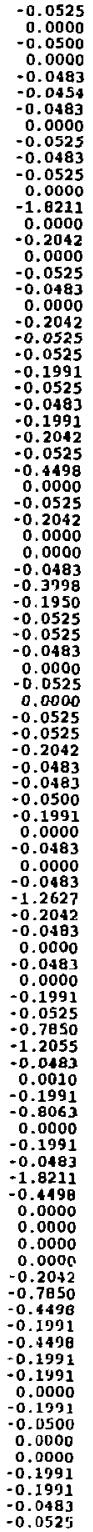 & 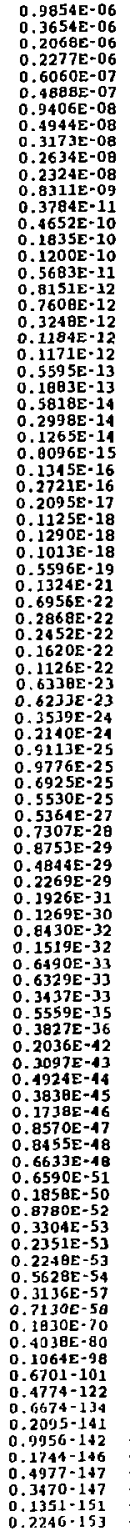 & 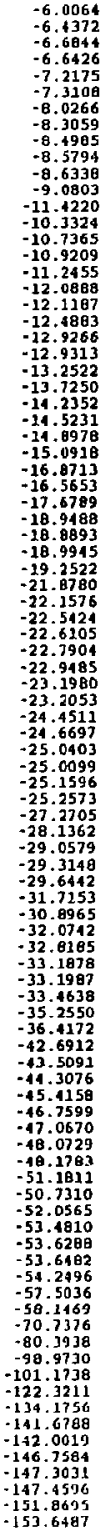 \\
\hline
\end{tabular}




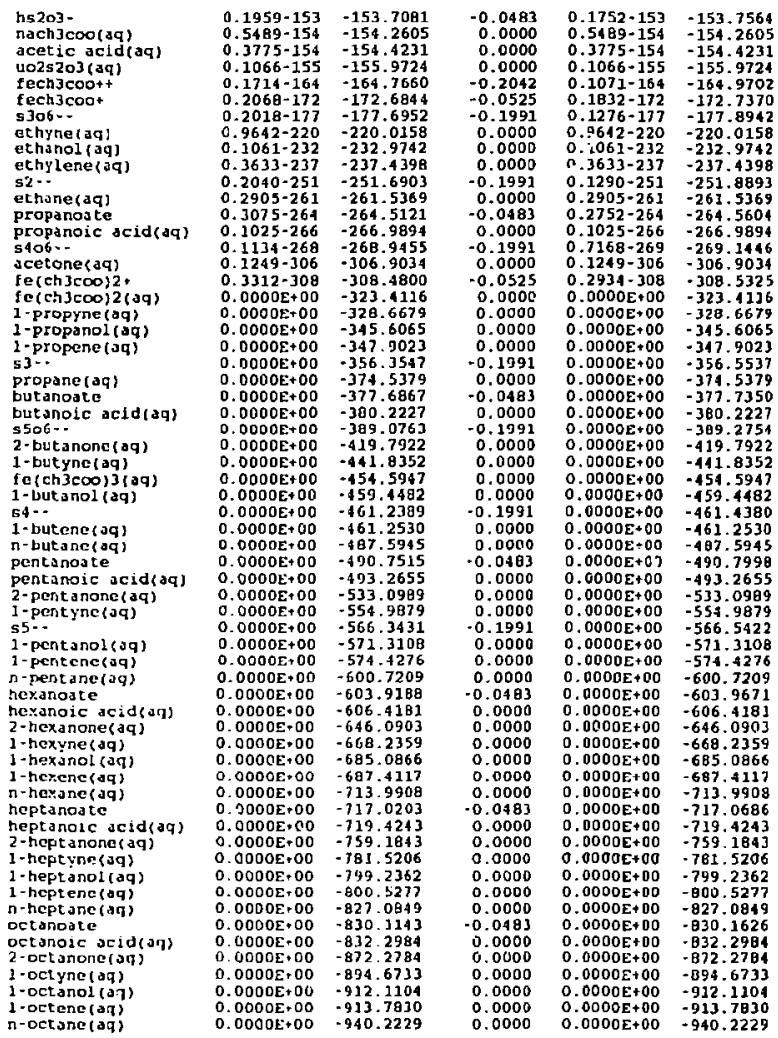

.... major aquepus species contributing to mass balances ....

aqueous species Jccounting for 992 or moro of ca*t

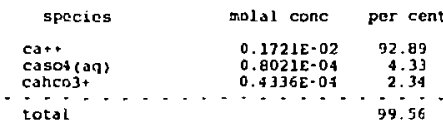

aquoous spectes decouneing for 998 or more of cl-

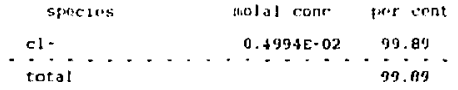

squeous species uccouneing for jog of more of $\mathrm{fot}$

species rolat conc por cont

fecoh) 3 (aq) $\quad 0.7 f_{1} 0$ HE- 12 BH. 45 


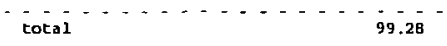

aqueous species accounting for 99 or more of hco3-

\begin{tabular}{|c|c|c|}
\hline species & molal conc & per cent \\
\hline 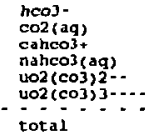 & $\begin{array}{r}0.3511 E-02 \\
0.3389 E-03 \\
0.4336 E-04 \\
0.2755 E-04 \\
0.2458 E-04 \\
0.1235 E-04\end{array}$ & $\begin{array}{r}87.33 \\
6.43 \\
1.08 \\
0.69 \\
1.22 \\
0.92 \\
-.0 . \\
99.67\end{array}$ \\
\hline $\begin{array}{c}\text { aqueous species } \\
\text { species }\end{array}$ & $\begin{array}{l}\text { accounting for } 998 \\
\text { molal cone }\end{array}$ & $\begin{array}{l}\text { more of } \\
\text { per cent }\end{array}$ \\
\hline$=\frac{n a+}{\operatorname{tocal}}=$ & $=-.+0.6942 \mathrm{E}-02$ & $\begin{array}{l}99.17 \\
99.17\end{array}$ \\
\hline
\end{tabular}

agueous species accounting for 991 or more of so4

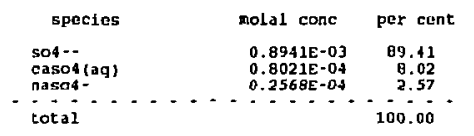

aqueous species accounting for 99 or more of us2t+

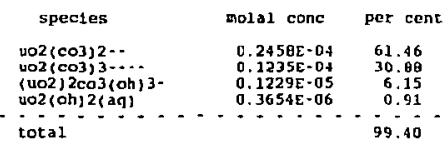

sumary of aqueous rcdox reactions .....

\begin{tabular}{|c|c|c|c|c|}
\hline couple & eh, volts & $p=-$ & 109 fod & ah, kcal \\
\hline 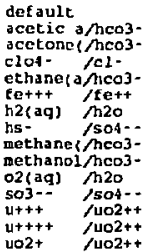 & $\begin{array}{l}0.786 \\
0.786 \\
0.786 \\
0.786 \\
0.786 \\
0.786 \\
0.786 \\
0.786 \\
0.786 \\
0.786 \\
0.786 \\
0.786 \\
0.786 \\
0.786 \\
0.786\end{array}$ & $\begin{array}{l}0.1329 \mathrm{E}+02 \\
0.1329 \mathrm{E}+02 \\
0.1329 \mathrm{E}+02 \\
0.1329 \mathrm{E}+02 \\
0.1329 \mathrm{E}+02 \\
0.1329 \mathrm{E}+02 \\
0.1329 \mathrm{E}+02 \\
0.1329 \mathrm{E}+02 \\
0.1329 \mathrm{E}+02 \\
0.1329 \mathrm{E}+02 \\
0.1329 \mathrm{E}+02 \\
0.1329 \mathrm{E}+02 \\
0.1329 \mathrm{E}+02 \\
0.1329 \mathrm{E}+02 \\
0.1329 \mathrm{E}+02\end{array}$ & $\begin{array}{l}-0.700 \\
=0.700 \\
=0.700 \\
-0.700 \\
=0.700 \\
-0.700 \\
-0.700 \\
-0.700 \\
-0.700 \\
-0.700 \\
-0.700 \\
=0.700 \\
=0.700 \\
-0.700 \\
-0.700\end{array}$ & $\begin{array}{l}18.132 \\
18.132 \\
10.132 \\
18.132 \\
18.132 \\
18.132 \\
18.132 \\
18.132 \\
18.132 \\
18.132 \\
18.132 \\
18.132 \\
18.132 \\
18.132 \\
18.132\end{array}$ \\
\hline
\end{tabular}

.... sumbary of aqueous non-equilibrium non-redox reactions ..... couple affinity, keal

none

.... sumary of stoichionetric mineral saturation states .....

\{minerals with iffinteies . . t , - $10 \mathrm{kcul}$ are not listed\}

\begin{tabular}{|c|c|c|c|c|c|c|c|}
\hline minera 1 & $\log q / x$ & aff, kcal & state & mineral & $\log q / k$ & aff $f, k \operatorname{col}$ & btate \\
\hline $\begin{array}{l}\text { anhydrite } \\
\text { bassanite } \\
\text { caso :0, } 5 \text { h2o(beta) } \\
\text { fefoh) } 3 \\
\text { Jypsun } \\
\text { herdtice } \\
\text { mirabilite }\end{array}$ & $\begin{array}{r}-1.900 \\
-2.545 \\
-2.714 \\
-5.602 \\
-1.725 \\
0.000 \\
-6.531\end{array}$ & $\begin{array}{r}.3 .593 \\
.3 .473 \\
-3.702 \\
-7.642 \\
-2.353 \\
0.000 \\
-8.911\end{array}$ & $5 a<d$ & $\begin{array}{l}\text { aragonite } \\
\text { calcite } \\
\text { caust } \\
\text { goethite } \\
\text { halite } \\
\text { ict } \\
\text { monohydrocolcite }\end{array}$ & $\begin{array}{r}-0.144 \\
0.000 \\
-0.499 \\
-0.480 \\
-6.248 \\
-0.139 \\
-0.834\end{array}$ & $\begin{array}{r}-0.197 \\
0.000 \\
-0.601 \\
-0.655 \\
-0.388 \\
-0.189 \\
-1.138\end{array}$ & $\begin{array}{l}\text { satd } \\
\text { satd }\end{array}$ \\
\hline
\end{tabular}




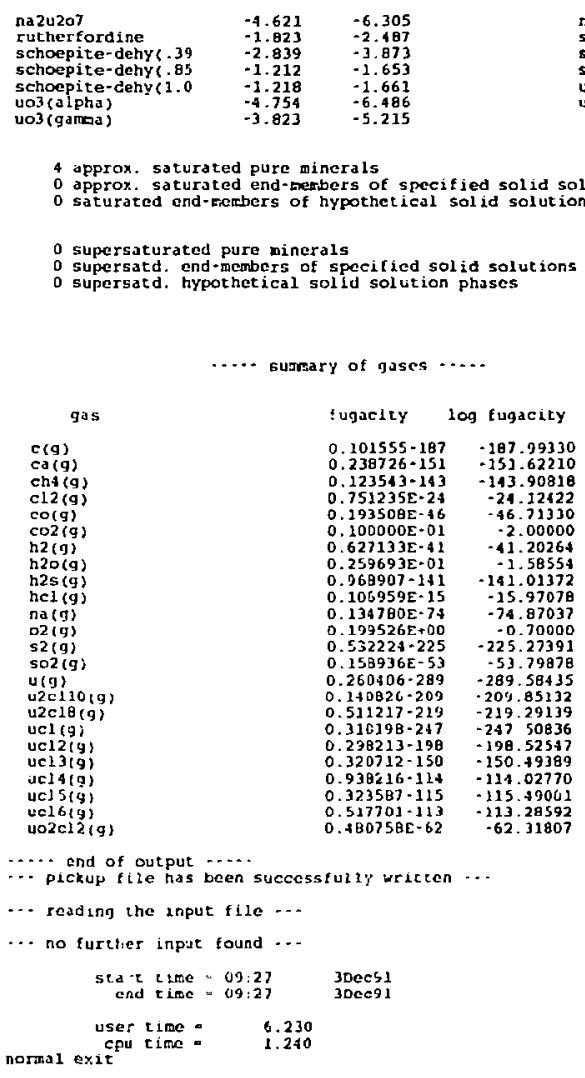

\subsection{Calculating the Composition of a Custom $p H$ Buffer: An Example}

This short example illustrates the use of EQ3NR to calculate the composition of a custom $p H$ buffer solution. Such buffers are highly useful in laboratory experiments, for example in determining the $p H$ dependence of mineral dissolution kinetics (e.g., Knauss and Wolery, 1986). The model is defined by choosing the desired $p H$ at the given temperature and the concentration of the buffering component. Electrical balancing is used to determine how much acid or base to include in the buffer recipe. The details are explained in the title on the input file, which is presented in both formats. This example was computed using the com data file. The activity coefficients were computed from the B-dot ejuation.

In this case, the buffer recipe is to consist of 0.05 molal boric and some unknown concentration of $\mathrm{NaOH}$. The $\mathrm{pH}$ is to be 8.00 at $70^{\circ} \mathrm{C}$. The desired concentration of $\mathrm{NaOH}$ is determined by 
electrical balancing on the sodium ion. The calculated concentration of $\mathrm{Na}^{+}$is 0.005691 molal. Hence the buffer should contain 0.005691 molal of $\mathrm{NaOH}$.

By doing very similar calculations with electrical balancing on the hydrogen ion, it is possible to confirm consistency between code calculations and standard $p H$ buffer recipes. The $p H$ of such solutions at other temperatures can then be found simply by changing the temperature on the data file. Some examples of this are given by Knauss and Wolery (1986).

When doing calculations involving buffers intended for use in the laboratory, it is always wise to check the saturation index results to ensure that the desired buffer composition is not supersaturated with respect to some solid phase. If it is, the buffer solution may be impossible to make up, or if it can be made up, it may not be stable owing to eventual precipitation of the supersaturated phase. The buffer solution may also be hard to make up if the solid form of the buffer component (such as boric acid) is undersaturated, but fairly close to saturation.

\section{The EQ3NR innut file (custombuf 3i) custom pH buffer test case ("W" format):}

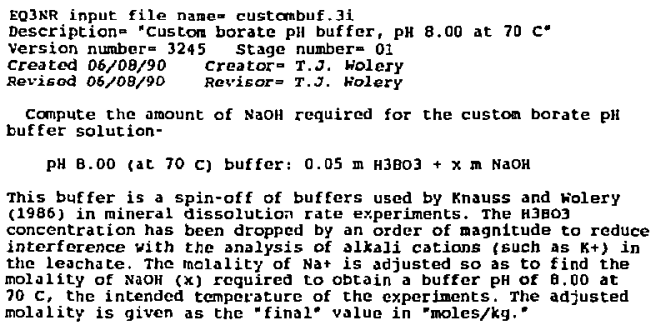

Purpose: to test electrical bslancing on a solute other than Ht and to demonstrate the coiln's ubilty to calculate the composition of a custom pil buffer.

Referenees

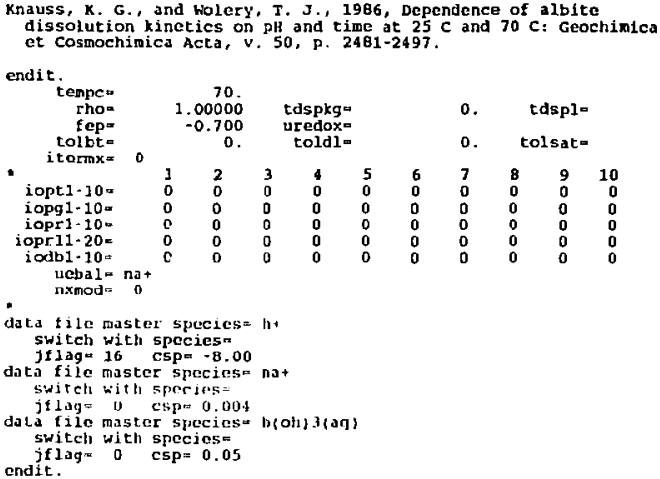




\section{The EQ3NR input file (custombut.3i),custom pH buffer test case (" $\mathrm{D}$ " format):}

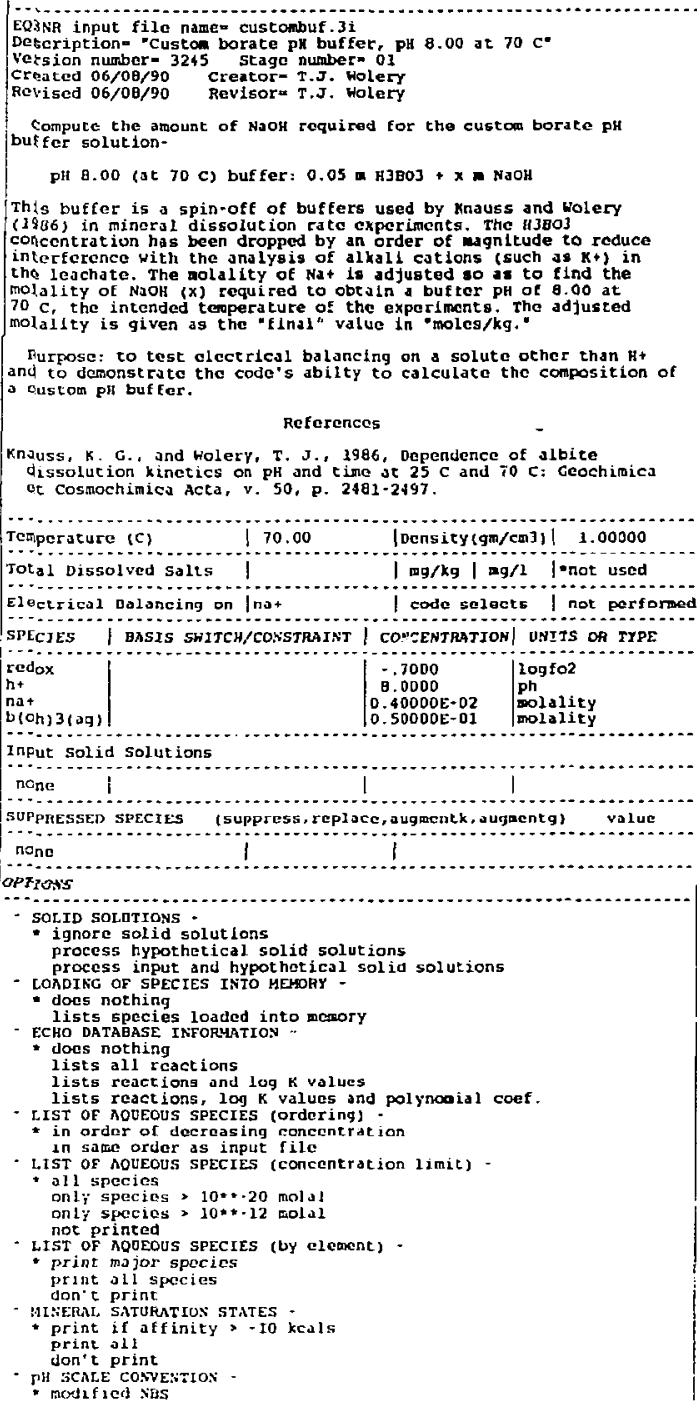


internal

rational

ACTIVITY Coefficient options -

* use B-dot equation

Davies' equation

Pitzcr's equations

AOTO BASIS SHITCHIYG

- ofe

- pitzer database inforhation -

- print only varnings

print species in model and number of pitzer coefficients

print species in wodel and nanes of pitzer coeffictents

PICXDP FILE

- write pickup Eile

don't write pickup file

- LIST MEAN IONIC PROPERTIES -

- don't print

print

ITST AQDEODS SDECIES, ION SIZES, AND HYDRATIOH MUHEERS -

print

don't print

ERITERIA

test both residual functions and correction toras

test only res iv'ul functions

DEBdGGING SWITCHES (0.off, 1,2-on, default lo off)

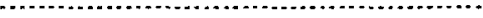

generlc debugging intolnacion

print details of pre-Neuton-Rophson 1 teration

- print details of verton-Raphson iteration

print detals of stoichlometric factors

0 Print details of stoichicetrio factors calculation
0 urite reactions on RLIST

o list stoichionetric coneentrations of master species

- request iteration variables to be killed

DEVELOPAENT OPTIONS (used for code development)

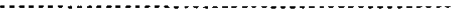

none TOLERAsces
(acsirgd values) (defaules)

residual functions correction terms saturation stato

number of $N-R$ iterations

The EQ3NR output file (custombuf:30), custom pH buffer test case (beginning with the message announcing the end of Newton-Raphson iteration):

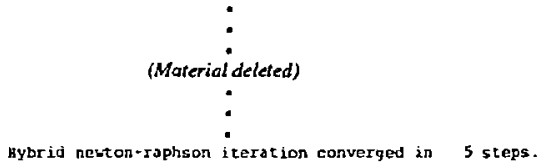

5 steps.

Summary of the Aqueous phase .....

Elenertal composition of the aqueous phase....
element
mg $/ 1$
mg/kg
noles/kg

$\begin{array}{ll}\text { o } & 0.09042 E+06 \\ b & 540.55 \\ \text { h } & 0.112035+06 \\ \text { na } & j 30.83\end{array}$

$0.89042 \mathrm{E}+O Q$

$\mathrm{ind} 5 \mathrm{~s}$

$0.11203 \mathrm{E}+\mathrm{OO}$

0.11114996372 .03

$0.5690539400 \mathrm{E}-02$

... eromertal composition as stracl basis species -...

species

h2o

bioh; $\geq: a q\}$ mig/l

$0.10026 \mathrm{E}+07$
3091.7

o. $11203 \mathrm{E}+0 \mathrm{G}$ mg/hg

$0.10026 E+07$

3091.7
$0.11203 \mathrm{E}+06$ moles/kg

$0.55653155 B 3 E+02$

$0.4999999993 \mathrm{E}-01$ 
... equivalent cosposition of the aqueous phase (cta balances) ... original basis existing basis

species

h2o
bioh) 3 (dq)

hat moles/kg h2o

$\begin{array}{ll}0.5565315583 \mathrm{E}+\mathrm{C2} & \mathrm{h} 20 \\ 0.4999999993 \mathrm{O}+01 & \mathrm{~b}(\mathrm{oh}) 3(\mathrm{aq}) \\ 0.111149963 \mathrm{E}+03 & \mathrm{ht} \\ 0.5690579408 \mathrm{H}-02 & \mathrm{nat}\end{array}$

noles/kg häo

$0.5565] 15583 \mathrm{E}+02$

$0.4999999993 \mathrm{E}-01$

$0.1111499637 \mathrm{E}+0 \mathrm{~J}$

$0.5690579408 \mathrm{E}-02$

single ion activities and activity coefficients are here defined with respect to tho modified nbs ph scale

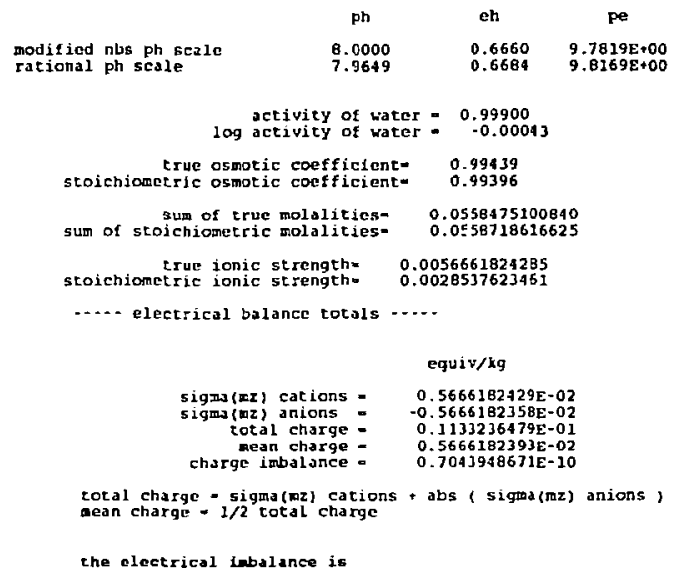

$0.622 E-06$ per cent of the total charge

$0.124 E-05$ per cent of the mean charge

$0.1245-05$ per cent of sigma(ta2) cations

$0.124 \mathrm{E}-05$ per cent of abs (sigan(mz) anions)

-. electrical balancing on na*

mg/kg

moles/kg

$\begin{array}{llll}\text { inout } & 91.959 & 91.959 & 0.4000000000 \mathrm{E}+02 \\ \text { final } & 130.83 & 130.83 & 0.5690579408 \mathrm{0}-02 \\ \text { atj } & 38.866 & 38.866 & 0.1690579408 \mathrm{0}-02\end{array}$

.... activity ratics of ions .....

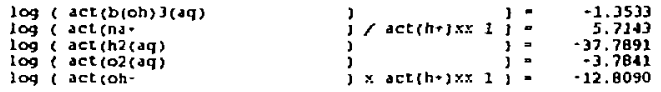

.... distrihution of aqueous species $\ldots$.

spmeles

b(oh) 3 (aq)

$\mathrm{nat}$

$\operatorname{tro} 2$.

02 (ง)

nabion $i(a q)$

oh-

naoh (aq)

h.

b2oions.

b) $303($ oh $) 4$. mols! conc Jay canc

$-1.3533$

-2. 2467

$0.4433 E-01$

0. $5666 \mathrm{E}-02$

o $56+9 \mathrm{~T}=02$

ง. $1643 \mathrm{E} \cdot 03$

$0.2435 \mathrm{E}-04$

$0.1693 \mathrm{E}-04$

o. $5624 E \cdot 07$

o. $10 \mathrm{e} 4 \mathrm{E} \cdot 0$ ?

o. $6786 \mathrm{E}-12$

2. 2400

$-3.7843$

$-4.6135$

$-4.7712$

$-7.2499$

$-7.9659$

$-12.1684$

$-15.5200$ loy $y$

0.0000

$-0.0390$

$-0.0369$

0.0001

0.0000

$-0.0378$

0.0000

$-0.0351$

$-0.0369$

-0.0369
-0.0369

act ivity

lay jet

$0.4433 E-01$ $0.5180 E-02$

TIRUE-0?

$0.16+4 E-03$

$0.2 \$ 35 E \cdot 04$

$0.1552 \mathrm{E}+04$

$0.5624 \mathrm{E}-07$

$0.1000 \mathrm{E}-07$

$0.6234 \mathrm{E}-12$

$0.6234 E-12$
$0.2769 E-15$

$-1.353$

$-2.2857$

$-2.2643$

$-3.2841$

$-4.6135$

$-4.8090$

$-7.2499$

$-8.0000$

$-12.2052$

$-15.5577$ 


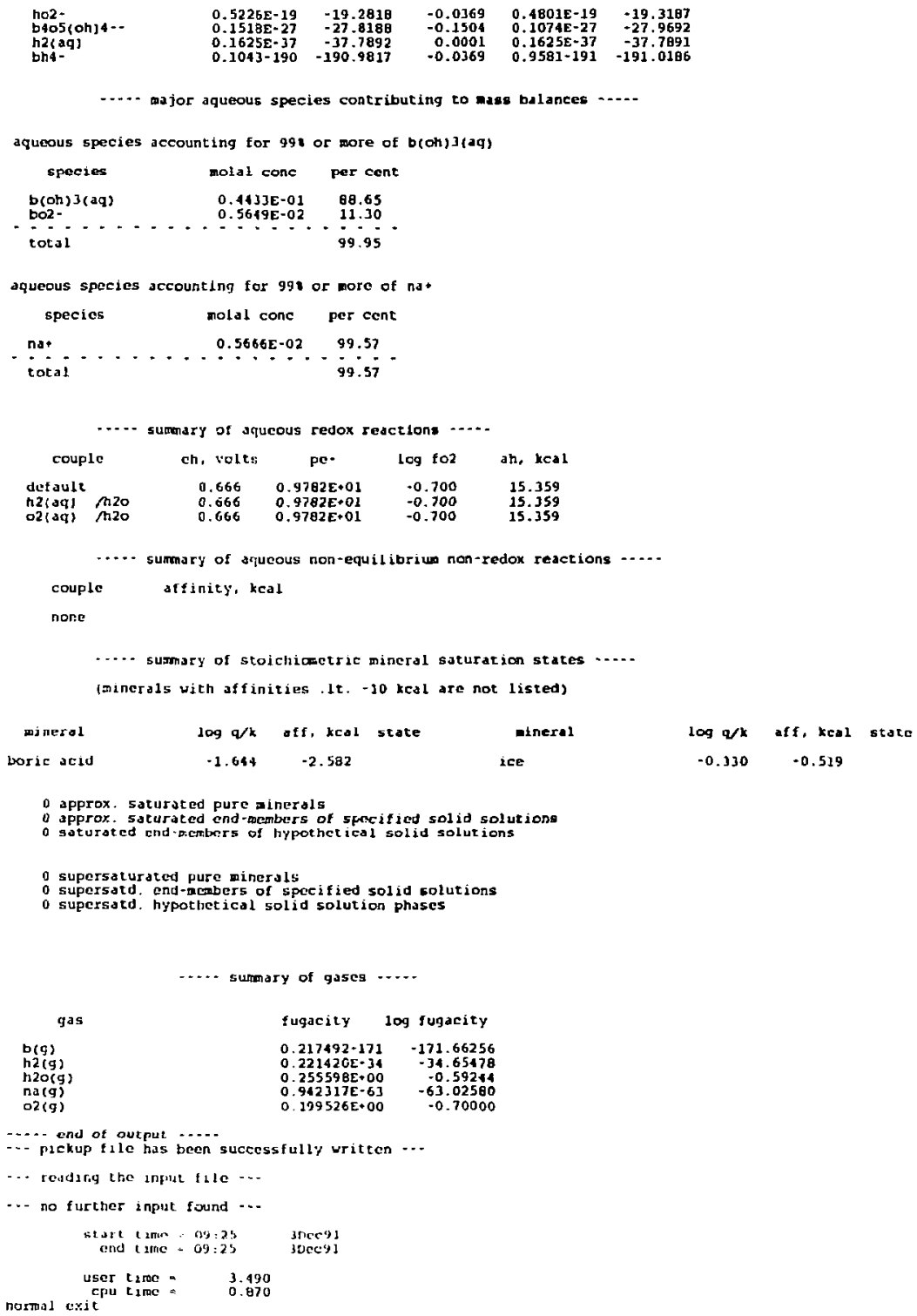




\subsection{Computing Oxygen Fugacity from Mineral Equilibria: An Example}

In this example, the oxygen fugacity of a hydrothermal solution at $250^{\circ} \mathrm{C}$ is estimated by assuming equilibrium between the aqueous solution, hematite, and magnetite. Note the use of the option iopt1 $=-3$, which opens up a species input block for $O_{2}(\mathrm{~g})$. Note that equilibrium with magnetite is the constraint assigned to $\mathrm{Fe}^{2+}$. Equilibrium with hematite is assigned to $\mathrm{O}_{2(\mathrm{~g})}$. This problem also determines the concentration of dissolved iron. This test case was adapted from one given by Henley et al. (1984), which also involves equilibrium with quartz, albite, K-feldspar, and muscovite, and electrical balancing to determine the $p H$. The results shown here were obtained using the com data file. The activity coefficients were computed from the B-dot equation. The input file is presented in both formats. The output file is presented beginning with the message announcing the end of Newton-Raphson iteration.

Equilibrium between hematite $\left(\mathrm{Fe}_{2} \mathrm{O}_{3(\mathrm{c})}\right)$ and magnetite $\left(\mathrm{Fe}_{3} \mathrm{O}_{4(\mathrm{c})}\right)$ fixes the oxygen fugacity because the reaction between the two can be written as:

$$
6 \mathrm{Fe}_{2} \mathrm{O}_{3(\mathrm{c})}=4 \mathrm{Fe}_{3} \mathrm{O}_{4(\mathrm{c})}+\mathrm{O}_{2(g)}
$$

Because the thermodynamic activities of the two minerals are each unity, the corresponding mass action equation reduces to:

$$
\log f_{\mathrm{O}_{2}}=\log K
$$

where $K$ is the equilibrium constant for the stated reaction.

Note that on the output file that the calculated saturation indices for hematite and magnetite are indeed zero. The $p H$ is -7.1045 , the $\log$ oxygen fugacity is -35.301 , and the concentration of dissolved iron is a very low $0.624 \times 10^{-9}$ molal.

The EQ3NR inout file (fo 2 minea.3i), the oxygen fugacity from mineral equilibria test case ("W" format):

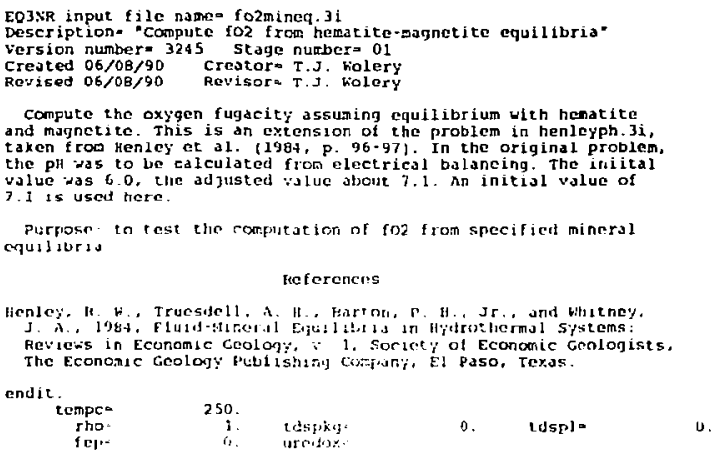




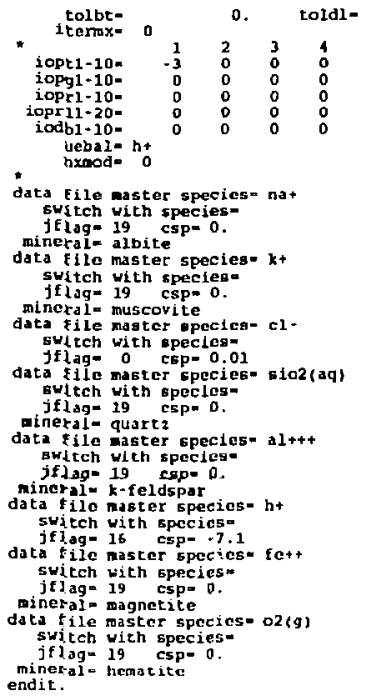

The EO3NR input file (fo2minea.3i), the oxygen fugacity from mineral equilibria test case ("D" format):

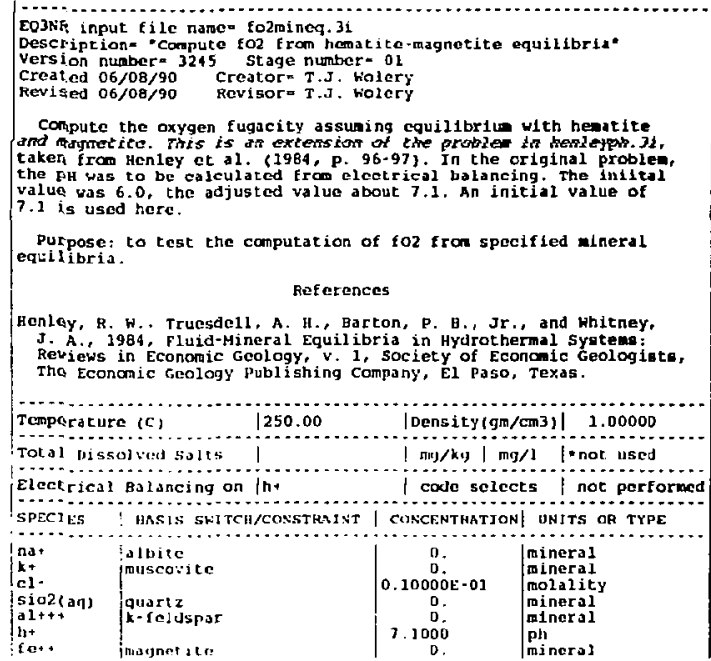

\begin{tabular}{|c|c|c|c|}
\hline $\begin{array}{l}\text { nat } \\
\text { kt } \\
\text { cl- } \\
\text { siog (arl) } \\
\text { alt+t } \\
\text { h+ } \\
\text { fo+t }\end{array}$ & 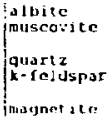 & $\begin{array}{c}0 . \\
D \\
0.10000 E-0] \\
0 \\
D \\
7.1000 \\
D .\end{array}$ & $\begin{array}{l}\text { mineral } \\
\text { wineral } \\
\text { molality } \\
\text { mineral } \\
\text { minoral } \\
\text { ph } \\
\text { mincras }\end{array}$ \\
\hline
\end{tabular}




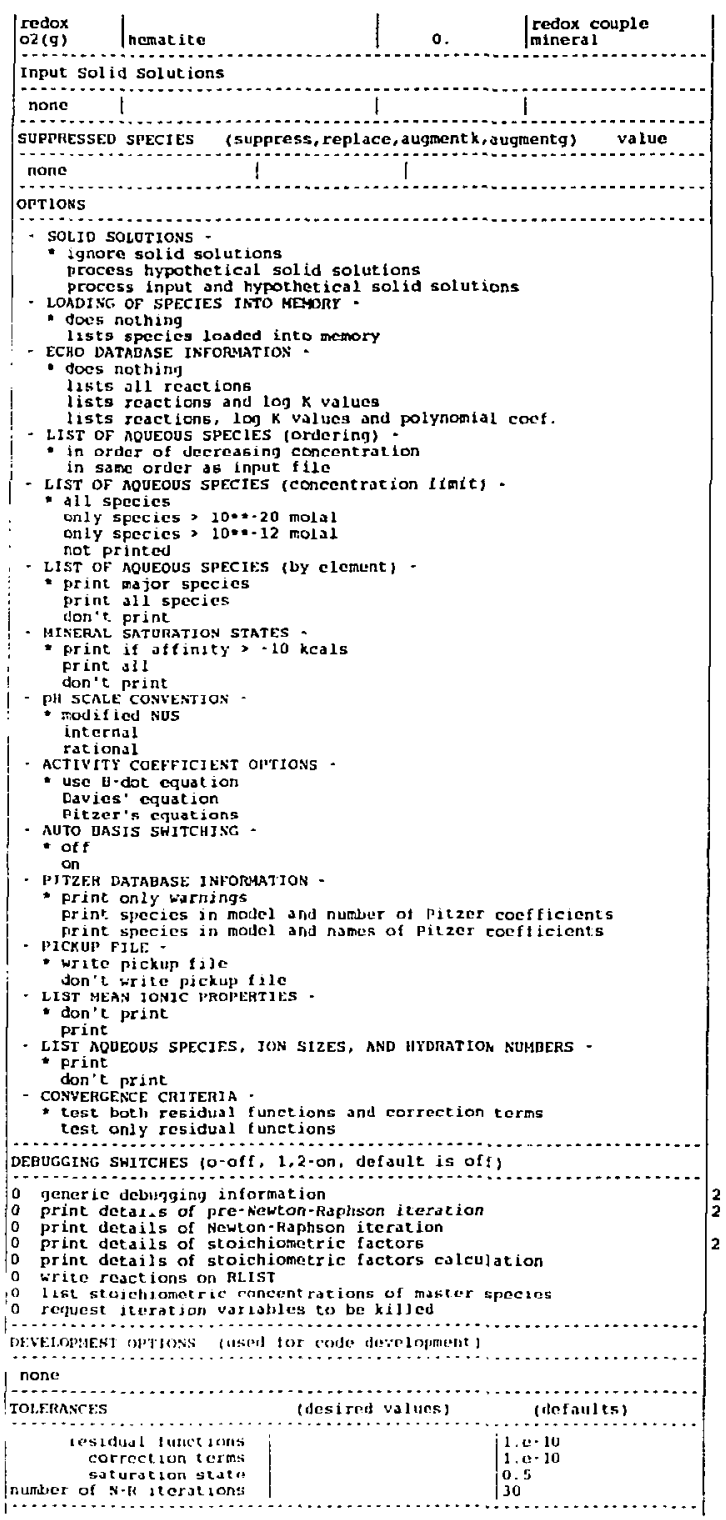


The EQ3NR ontput file ( 102 minen 30 ), the oxygen fugacity from mineral equilibria test case (beginning with the message announcing the end of Newton-Raphson iteration):

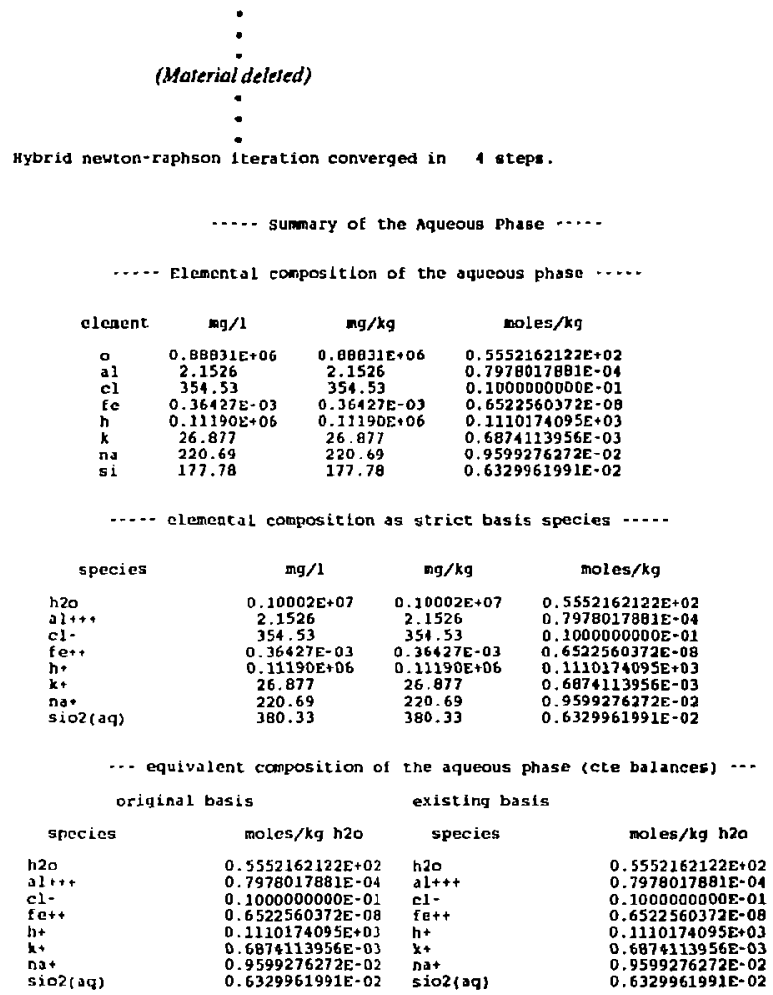

single ion activities and activity coeficients are here defined with respect to the modified nbs ph scale

$\begin{array}{cccc} & \text { ph } & \text { ch } & \text { pe } \\ \text { rodified nbs ph scale } & 7.1043 & -0.4109 & -4.2479 E+00 \\ \text { rational ph scale } & 7.0303 & -0.4333 & -4.1739 E+00\end{array}$

phe1 = 9.1965

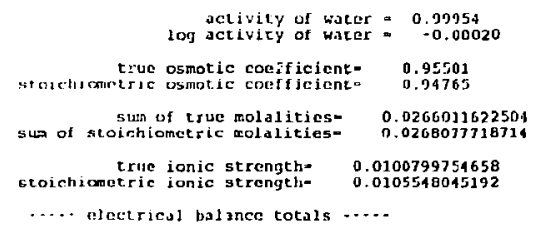




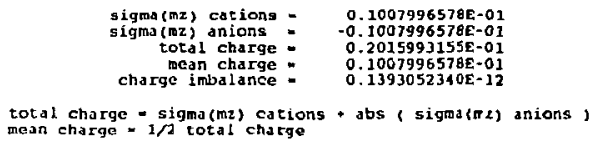

mean charge $=1 / 2$ total charge

the electrical Labalance is

$0.691 \mathrm{E}-09$ per cent of the total charge

$0.136 \mathrm{E}-08$ por cent of the mean chargo

$0.138 \mathrm{E}-08$ per cent of abs (

.. electricjl balaneing on $\mathrm{ht}$

$\log$ activity

$\begin{array}{ll}\text { inpue } & -7.1000 \\ \text { final } & -7.1043 \\ \text { adj } & -0.42640 \mathrm{E}-02\end{array}$

.... activity ratios of ions ....
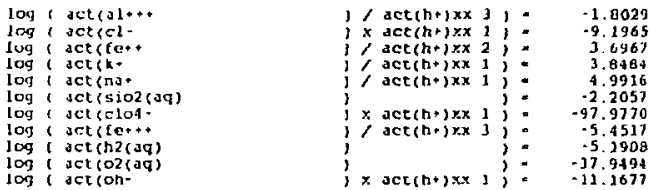

.... distribution of aqueous species -...

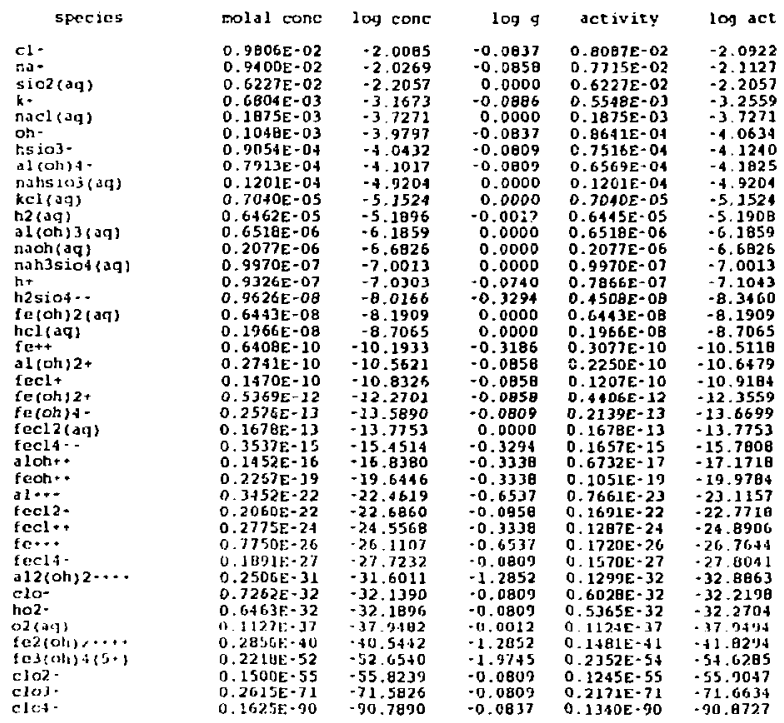


aqueous species accounting for 991 or more of alt++

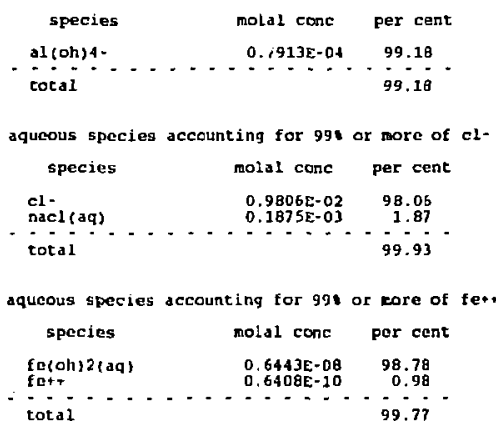

aqueous species accounting for 99 ar more of $k$ *

\begin{tabular}{lrr} 
species & molal conc & per cent \\
k+ & $0.6804 E-03$ & 98.98 \\
kel (aq) & $0.7040 E-05$ & 1.02 \\
\hline total &...+ & -.00
\end{tabular}

squeous species accounting for 99 or more of nat

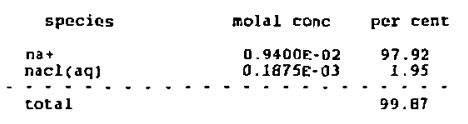

aqueous species accounting for 998 or nore of sio2(aq)

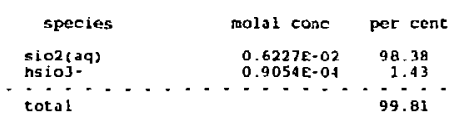

-... summary of aqueous sedox reactions -...

\begin{tabular}{|c|c|c|c|c|c|}
\hline coupl & & en, volts & pe- & $\log \mathrm{for}$ & $a h$, xeal \\
\hline $\begin{array}{l}\text { default } \\
\text { clot- } \\
\text { fet+t } \\
\text { h2(aq) } \\
\text { o2(aq) }\end{array}$ & $\begin{array}{l}/ c 1- \\
/ 1 \mathrm{c}+t \\
\operatorname{h2o} \\
\operatorname{h} 20\end{array}$ & $\begin{array}{l}-0.441 \\
-0.441 \\
-0.441 \\
-0.441 \\
-0.441\end{array}$ & $\begin{array}{l}-0.4248 E+01 \\
=0.4248 E+01 \\
-0.4248 E+01 \\
-0.4248 E+01 \\
-0.4248 E+01\end{array}$ & $\begin{array}{l}-35.301 \\
-35.301 \\
-35.301 \\
-35.301 \\
-35.301\end{array}$ & $\begin{array}{l}-10.169 \\
-10.169 \\
-10.169 \\
-10.169 \\
-10.169\end{array}$ \\
\hline
\end{tabular}

.... sumnary of aqueous non-equilibriun non-redox reactions .... couple affinity, kcal

nane

.... sumariry of stoichicmetric mineral saturation states ....

(minerals with affinities .2t. - 10 kcol are not listed)

mineral

albite

albite low analeime-dehy onnite
beldellite-k

\begin{tabular}{|c|c|c|}
\hline $\log 9 / k$ & $J \in E, k, \mathbb{J} L$ & StJie \\
\hline $\begin{array}{r}0.000 \\
0.000 \\
-3.475 \\
-0.767 \\
-1.277\end{array}$ & $\begin{array}{r}0.000 \\
-0.001 \\
-8.318 \\
-1.837 \\
-3.057\end{array}$ & $\begin{array}{l}\text { satd } \\
\text { satd }\end{array}$ \\
\hline
\end{tabular}

mineral

albite high and leime andalusite beidellite-h
beidell te-no $\log 9 / x$

$-0.490$

$-0.388$

$-2.190$

$-1.606$

$-0.951$ aff. keal stato

-1. 173

$-0.929$

$-5.241$

-3.644
-2.277 


\begin{tabular}{|c|c|c|c|c|c|c|c|}
\hline $\begin{array}{l}\text { boehmite } \\
\text { chamosite-7a } \\
\text { coesite } \\
\text { cristobulite } \\
\text { cristobalite-b } \\
\text { daphnite- } 4 \text { a } \\
\text { fayalite } \\
\text { fea } \\
\text { gibbsite } \\
\text { greenalite } \\
\text { hercynite } \\
\text { jadeite } \\
\text { kalsilite } \\
\text { kyanite } \\
\text { maximum microcline } \\
\text { muscovite } \\
\text { nepheline } \\
\text { nontronite-k } \\
\text { paragenite } \\
\text { quatz } \\
\text { sillmanite } \\
\text { fridyite }\end{array}$ & $\begin{array}{l}-1.095 \\
-2.151 \\
-0.489 \\
-0.266 \\
-0.441 \\
-1.927 \\
-1.606 \\
-1.738 \\
-1.501 \\
-2.242 \\
-1.541 \\
-1.643 \\
-1.730 \\
-2.243 \\
0.015 \\
0.000 \\
-1.685 \\
-0.660 \\
-0.641 \\
0.000 \\
-2.319 \\
-0.371\end{array}$ & $\begin{array}{r}-2.621 \\
-5.148 \\
-1.170 \\
-0.636 \\
-1.056 \\
-4.614 \\
-3.845 \\
-4.160 \\
-3.594 \\
-5.367 \\
-3.689 \\
-3.934 \\
-4.141 \\
-5.370 \\
0.035 \\
0.000 \\
-4.033 \\
-1.580 \\
-1.535 \\
0.000 \\
-5.551 \\
-0.889\end{array}$ & $\begin{array}{l}\text { sucd } \\
\text { satd }\end{array}$ & 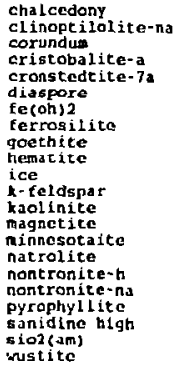 & $\begin{array}{r}-0.155 \\
-3.443 \\
-2.664 \\
-0.266 \\
-1.650 \\
-0.909 \\
-3.085 \\
-0.819 \\
-1.435 \\
0.000 \\
-1.122 \\
0.000 \\
-1.313 \\
0.000 \\
-0.877 \\
-2.493 \\
-0.985 \\
-0.336 \\
-1.490 \\
-0.349 \\
-0.51 .7 \\
-1.992\end{array}$ & $\begin{array}{r}-0.370 \\
-0.241 \\
-6.378 \\
-0.636 \\
-3.949 \\
-2.176 \\
-7.385 \\
-1.962 \\
-3.434 \\
0.000 \\
-2.686 \\
0.000 \\
-3.144 \\
0.000 \\
-2.100 \\
-5.968 \\
-2.359 \\
-0.805 \\
-3.568 \\
-0.036 \\
-1.228 \\
-4.768\end{array}$ & $\begin{array}{l}\text { satd } \\
\text { satd } \\
\text { satd }\end{array}$ \\
\hline
\end{tabular}

9 approx. soturated pure minerals

0 approx. saturated end-meahers of specified solid solutions

0 saturated end-members of hypothetical solid solutions

O supersaturated pure minerals

0 supersatd. end-merbers of specified solid colutions

o supersatd. hypothetical solid solution pliases

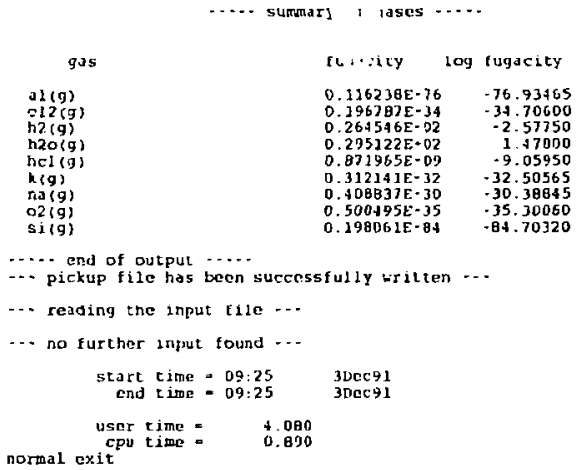

\subsection{Computing Eh from a Redox Couple: An Example}

This test case illustrates the computation of Eh (or pe, oxygen fugacity, or $A h$ ) from data for both members of a redox couple. The fluid is an acid $(p H=1.1)$ mine water whose composition is taken from Nordstrom, Jenne, and Ball (1979). The redox state is calculated for the $\mathrm{Fe}^{2+}-\mathrm{F} e^{3+}$ couple. This is possible because the concentration of each form of dissolved iron is sufficiently high to be measured. The objective is to compare the Eh calculated for this couple with the measured $E h$ of $+622 \mathrm{mV}$. This problem was run using the com data file, and the activity coefficients are calculated from the B-dot equation. The input file in both formats is given below, followed by the output file, beginning with the message announcing the completion of Newton-Raphson iteration. 
In this particular case, the measured $E h$ of $+622 \mathrm{mV}$ was used as the default redox parameter. This was accomplished by setting iopt $1=-1$ and $f e p=0.622$. This was used to constrain all the redox couples in the solution, except that for $\mathrm{Fe}^{2+}-\mathrm{Fe}^{3+}$. For the latter couple, a separate analytical concentration was entered for each member, and the corresponding redox state then calculated. Of particular interest is the following table:

\begin{tabular}{|c|c|c|c|c|}
\hline couple & eh, volts & pe- & $\log 102$ & ah, kcal \\
\hline $\begin{array}{l}\text { default } \\
\text { fe+t+ } / \text { fo+* } \\
\text { h2(aq) /h2o } \\
\text { hs- } / 804= \\
\text { o2(aq) //20 } \\
\text { so3-- /so4-. }\end{array}$ & $\begin{array}{l}0.622 \\
0.718 \\
0.622 \\
0.622 \\
0.622 \\
0.622\end{array}$ & $\begin{array}{l}0.1050 E+02 \\
0.1212 \mathrm{E}+02 \\
0.1050 \mathrm{0}+02 \\
0.1050 \mathrm{0}+02 \\
0.1050 \mathrm{0}+02 \\
0.1050 \mathrm{0}+02\end{array}$ & $\begin{array}{l}-36.590 \\
-30.115 \\
-36.590 \\
-36.590 \\
-36.590 \\
-36.590\end{array}$ & $\begin{array}{l}14.345 \\
16.557 \\
14.345 \\
14.345 \\
14.345 \\
14.345\end{array}$ \\
\hline
\end{tabular}

Here we see that the redox state of the ferrous-ferric couple expressed as $E h$ is $+718 \mathrm{mV}$, higher than the measured value of $+622 \mathrm{mV}$. The $E h$ of all other redox couples matches the default value. If we had set iopgl $=1$ and uredox $=$ 'fe +++ ', the default redox state would have been determined by that for the ferrous-ferric couple instead.

Is the difference between the computed $E h$ for the ferrous-ferric couple and the measured $E h$ significant? This is not immediately obvious. To pursue this question, one might like to know the likely error in the measured $E h$. One might also like to estimate the uncertainty in the calculated $E h$ of the ferrous-ferric couple due to the analytical uncertainties in the measurement of the concentration of the two forms of dissolved iron. One might also like to estimate the component of uncertainty in this quantity arising from uncertainty in the measurement of the $p H$. Recall that the reported $p H$ value was 1.1. Calibration buffers are generally available for $p H$ values of about $4.0,7.0$, and 10.0. Thus it is likely that the measurement involved considerable extrapolation, and the true uncertainty is probably at least a few tenths of a $\mathrm{pH}$ unit. Note also that the calculated charge imbalance for the total water analysis on the high side ( $-16 \%$ of the total ionic charge). In addition to those considerations, uncertainty in the calculated $E h$ also arises from uncertainties in the thermodynamic data and the activity coefficients. Nordstrom, Jenne, and Ball (1979) were partly able to get around such problems by plotting the ferrous-ferric $E \boldsymbol{h}$ versus the measured $E \boldsymbol{h}$ for a suite of such waters.

Although this example involves the additional data required to evaluate the redox state of only one couple, data may be specified to allow the determination of the redox states of any number of redox couples. Generally speaking, two analytical data inputs are required per couple. However, if water is one of the members of a couple, only an analytical data input for the other member is required.

The EO3NR innut file (acidmwh,3i), the redox couple test case ("W" format):

EO3NR input file name= acidmill. $3 \mathrm{i}$

Descript ion" "Acid mine watur. Hornet Effluent"

version number $=3245$ stage numiber $=01$

created $06108 / 90$ creatora num. Holery

Revised 06/00/90 Revisor $=$ T.J. Holery

Acid nine water, Hornet Effluent. Analys is from Nordstrom, Jenne,

and Ball 11979 , Table $[F$, colunn $B$ ). Note that separate analyses are

present for Fet+ and Fett+, permitting the cal-ulation of the Eh

specific to this couple. This may then be exmpared with the measured 
Eh.

Purpose: to test the code in the case of an acid mine water.

.

Feferences

Wardstram, D. K., Jerne, E. A., and ba11, J. H., 1979, Redox

cqullibria of iron in acid aine waters, in Jenne, E. A. editor, Chemical Modeling in Aqueous systens, Acs Symposiua Series,

v. 93, Averican Clemical Society, Hashington, D.C., p. 51-79.

endit.

ierope.

rho:

fepa

talbe:

iterax:-0

iopt -10.

lopgl-10.

ioprd-10.

iopr $12-20$.

iopr $11-20=$
iodbl-10-

vebal- none

nxmode 0

data file master species- ho

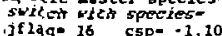

data fije master species: $\mathrm{co+t}$ suitech vith species=

$j f 1 \mathrm{ag}=2$ csp $=173$.

data file master species- nagt+ switch with species-

jflag: 2 csp= 605 .

daca file master species $=$ na+

suikch with species:

jf $10 g^{\circ} 2$ csp 92.5

dab file auster species= $k+$

sitich with species=

jflaq= 2 csp= 128.

data filc master species $=$ fett

switch with species=

data file mister species= fet+4

switch with species=

jflag= 2 csp- 2650 .

data file muster species* al + .

stiteh with species:

jflag 2 cSt $=1400$.

data file rostier species= sioz(aq)

switch with species:

jflag- 2 , csp $=130$

data file master species= so4-.

switch with species:

endit.

jflag- 2 csp= 60000 .

he EQ3NR input file (acidmwh.3i), the redox couple test case ("D" format):

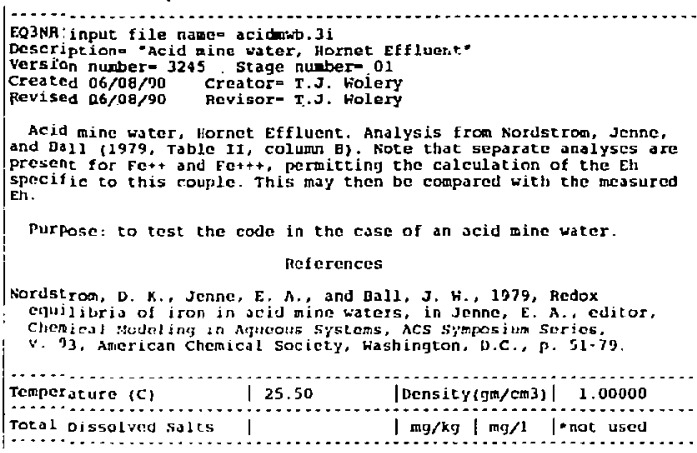




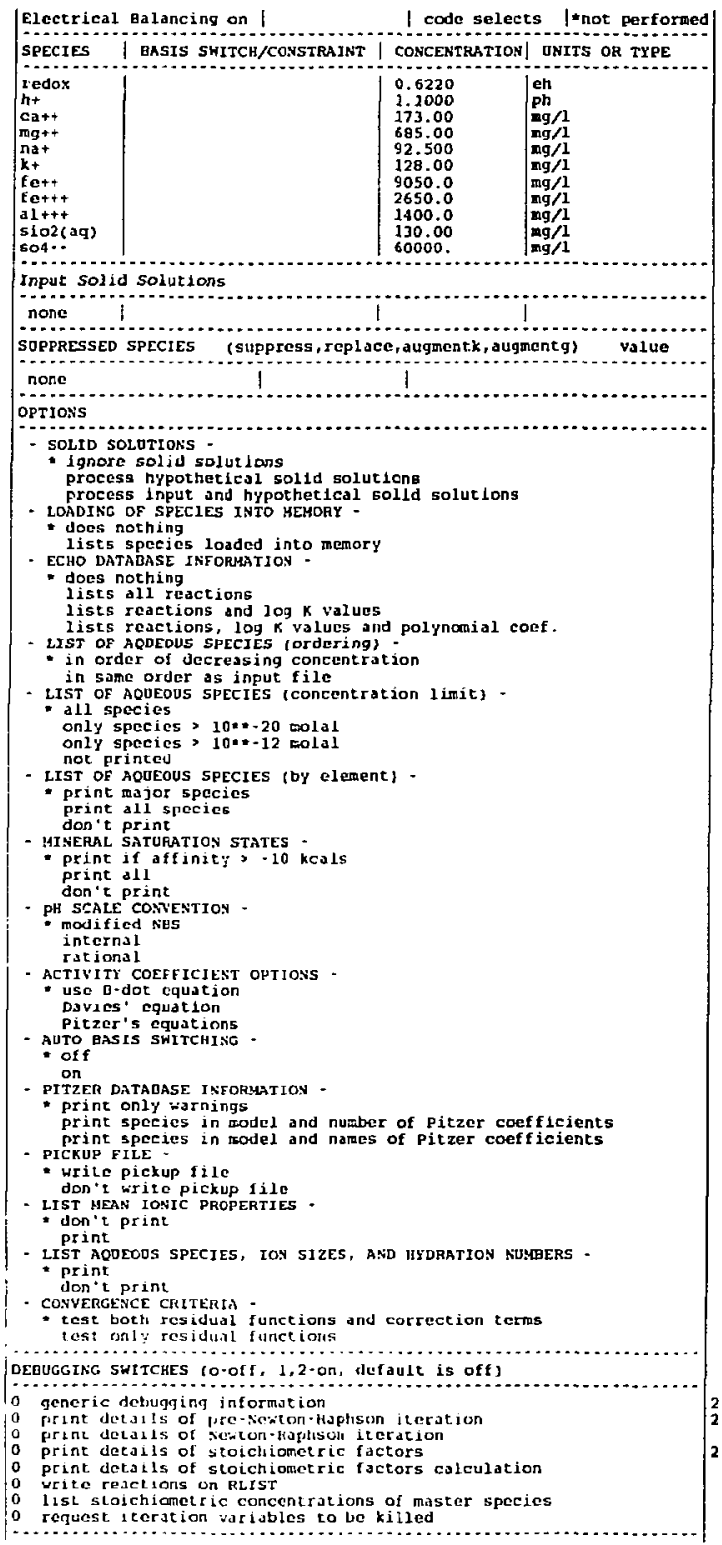




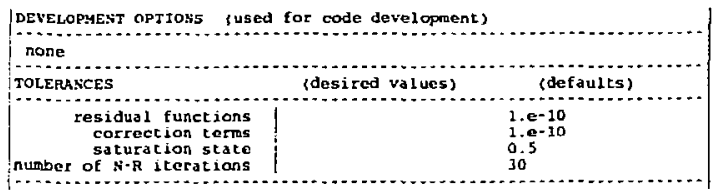

The EQ3NR output file (acidmwb.30) the redox couple test case (beginning with the message announcing the end of Newton-Raphson iteration):

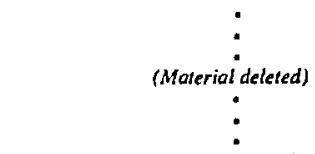

Hybrid neiton-raphson iteration converged in 7 :eps.

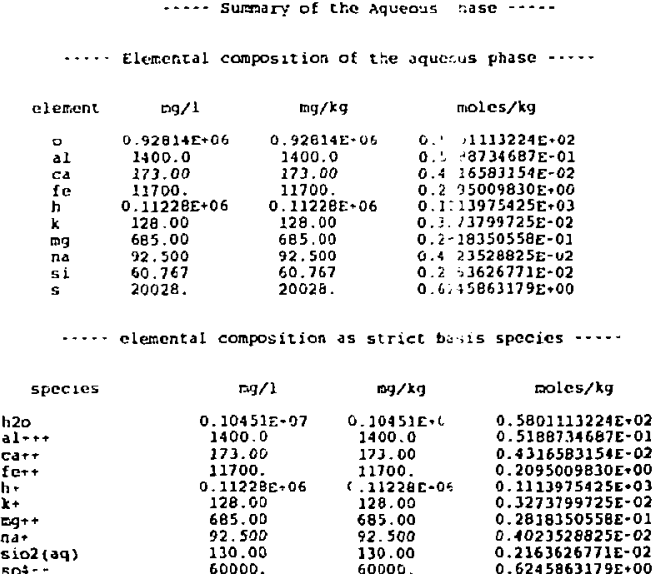

-. equivalent composicion of the aqueoss phase (cte balances) -.. original basis

extsting basis

species

noles/kg h2o

$0.5801113224 E+02$

$0.5188734687 \mathrm{E}-01$

$0.4316593154 \mathrm{~F}=02$

$0.43165831545=02$

1.. $15204930691-00$

$0.1115975425 E-03$

0. $3273799725 \mathrm{E}-02$

$0.2818350558 \mathrm{E}-01$

$0.4023528825 E \cdot 02$

0. $211,31,20771 \mathrm{E}-63$

6. $5245653175 \mathrm{~F}+00$

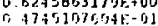

specif

n2o

a $1+\cdots$

cat

h.

my.

mi *

$5.02(\mathrm{n} /)$.

. 04. moles/kg h2o

$0.5801113224 E+02$

$0.5188734687 \mathrm{TE}-02$

$0.43165831546-02$

$0.16204990696+06$

$0.1620459069 E+06$
$0.1113975+25 E+01$

$0.3273799725 E-02$

$0.2818350558 E-01$

$0.5123528825 \mathrm{SE} .02$

$0.2163626771 \mathrm{E} .02$

$6.6245863179 \mathrm{BE}+00$

1. $474520760.5+0$ ?

single ion actiojics and activity coefficients are here defined

iIth respect to enn nodified nbs ph seale 


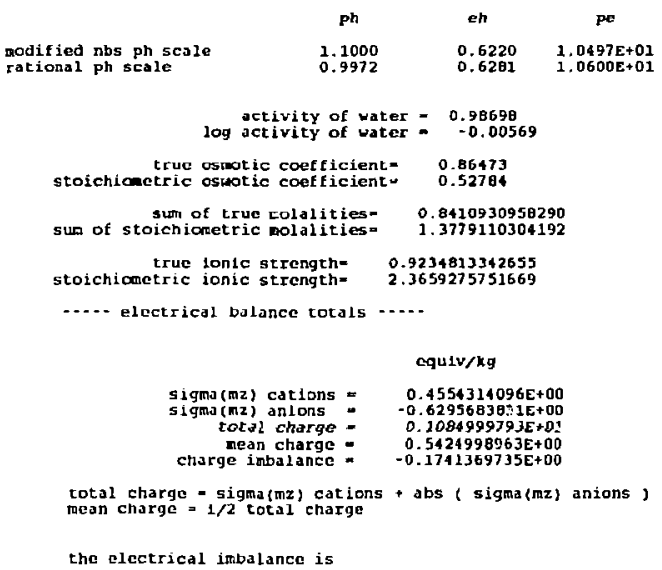

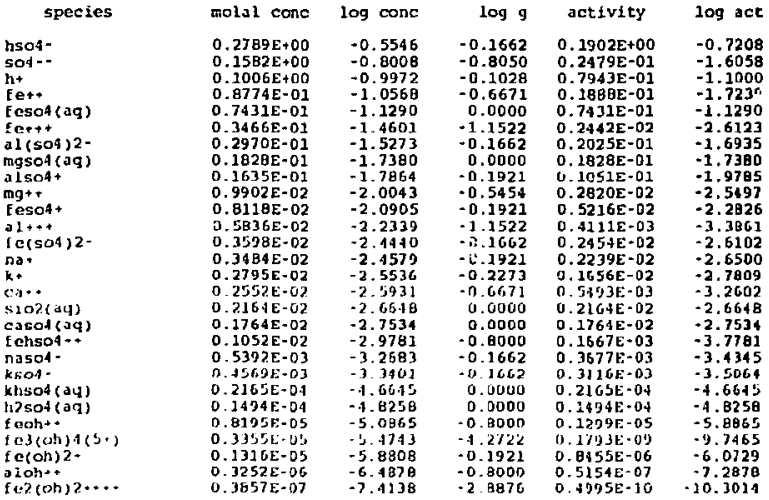




\begin{tabular}{|c|c|c|c|c|c|}
\hline 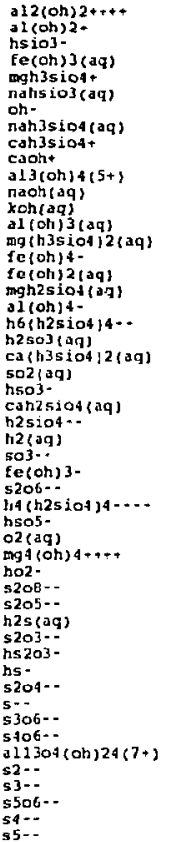 & 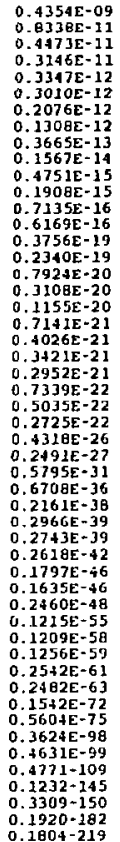 & $\begin{array}{l}-9.3611 \\
-11.0789 \\
-11.3494 \\
-11.5022 \\
-12.4754 \\
-12.5214 \\
-12.6928 \\
-12.8633 \\
-13.4359 \\
-14.0049 \\
-15.3232 \\
-15.7193 \\
-16.1466 \\
-16.2098 \\
-19.4253 \\
-19.6308 \\
-20.1010 \\
-20.5075 \\
-20.9375 \\
-21.1462 \\
-21.3951 \\
-21.4659 \\
-21.5298 \\
-22.1344 \\
-22.2980 \\
-22.5647 \\
-26.3647 \\
-27.6036 \\
-31.2369 \\
-36.1734 \\
-38.6654 \\
-39.5279 \\
-39.5617 \\
-42.5821 \\
-46.7454 \\
-46.7665 \\
-48.6090 \\
-55.9154 \\
-58.9177 \\
-59.9011 \\
-61.5949 \\
-63.6051 \\
-72.8120 \\
-75.2515 \\
-98.4408 \\
-99.3344 \\
-39.3224 \\
-145.9092 \\
-150.4803 \\
-182.7167 \\
-219.7437\end{array}$ & $\begin{array}{r}-2.8876 \\
-0.1921 \\
-0.1662 \\
0.0000 \\
-0.1921 \\
0.0000 \\
-0.2015 \\
0.0000 \\
-0.1921 \\
-0.1921 \\
-4.2722 \\
0.0000 \\
0.0000 \\
0.0000 \\
0.0000 \\
-0.1662 \\
0.0000 \\
0.0000 \\
-0.1662 \\
-0.8050 \\
0.0000 \\
0.0000 \\
0.0000 \\
-0.1652 \\
0.0000 \\
-0.8050 \\
0.0761 \\
-0.8050 \\
-0.1652 \\
-0.809 \\
-3.3611 \\
-0.1611 \\
0.0761 \\
-2.8876 \\
-0.1652 \\
-0.8050 \\
-0.8050 \\
0.0000 \\
-0.800 \\
-0.16 .2 \\
-0.2015 \\
-0.8050 \\
-0.8050 \\
-0.6050 \\
-0.8050 \\
-0.3730 \\
-0.005 i \\
-0.8750 \\
-0.6050 \\
-0.8050 \\
-0.9050\end{array}$ & $\begin{array}{l}0.5640 E-12 \\
0.5357 E-11 \\
0.3050 E-11 \\
0.3146 E-11 \\
0.2150 E-12 \\
0.3010 E-12 \\
0.1305 E-12 \\
0.1308 E-12 \\
0.2355 E-13 \\
0.1007 E-11 \\
0.2539 E-19 \\
0.1900 E-15 \\
0.7135 E-16 \\
0.6169 E-16 \\
0.3756 E-19 \\
0.1536 E-19 \\
0.7924 E-20 \\
0.3108 E-20 \\
0.7875 E-21 \\
0.1119 E-21 \\
0.4026 E-21 \\
0.3421 E-21 \\
0.2952 E-21 \\
0.5005 E-22 \\
0.50] 5 E-22 \\
0.4269 E-23 \\
0.5145 E-26 \\
0.3903 E-28 \\
0.3952 E-31 \\
0.1051 E-36 \\
0.888 B E-42 \\
0.2023 E-39 \\
0.3269 E-39 \\
0.3190 E-45 \\
0.1226 E-46 \\
0.2562 E-47 \\
0.3655 E-49 \\
0.1215 E-55 \\
0.1894 E-59 \\
0.8565 E-60 \\
0.1598 E-61 \\
0.3839 E-64 \\
0.2416 E-73 \\
0.8760 E-76 \\
0.5679 E-99 \\
0.1958-107 \\
0.7476-110 \\
0.1931-146 \\
0.5165-151 \\
0.3009-183 \\
0.2827-220\end{array}$ & $\begin{array}{r}-12.2497 \\
-11.2710 \\
-11.5156 \\
-11.5022 \\
-12.6675 \\
-12.5214 \\
-12.0843 \\
-12.0833 \\
-13.6280 \\
-14.9970 \\
-19.5954 \\
-15.7193 \\
-16.1466 \\
-16.2098 \\
-19.4253 \\
-19.7970 \\
-20.1010 \\
-20.5075 \\
-21.1037 \\
-21.9512 \\
-21.3951 \\
-21.4658 \\
-21.5298 \\
-22.3006 \\
-22.2980 \\
-23.3696 \\
-26.2866 \\
-28.4086 \\
-31.4032 \\
-36.9784 \\
-42.0512 \\
-39.6941 \\
-39.4856 \\
-45.4697 \\
-46.9117 \\
-47.5915 \\
-49.4140 \\
-55.9154 \\
-59.7226 \\
-60.0673 \\
-61.7963 \\
-64.4101 \\
-73.6170 \\
-76.0565 \\
-99.2457 \\
-107.7091 \\
-110.1263 \\
-146.3142 \\
-151.2852 \\
-183.5217 \\
-220.5466\end{array}$ \\
\hline
\end{tabular}

.... rajor aquaous spocios contributing to mass balances ....

aqueous species accounting for 998 or more of al+*+

species molal cone per cent

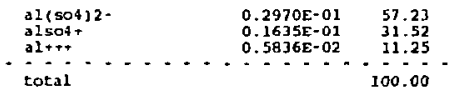

aqueous species accounting for 991 or more of ca+*

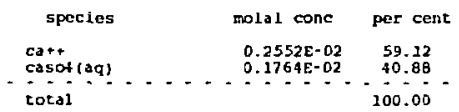

aqueous specics accounting for $39 t$ or more of fet+

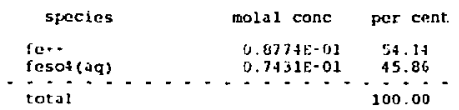

aqueaus species accounting for 992 or more of $k+$

$\begin{array}{lcc}\text { species molal conc } & \text { per cant } \\ \text { k. } & 0.2795 \mathrm{E}-02 & \text { B5. 38 } \\ \text { ksat- } & 0.4569-03 & 13.96\end{array}$




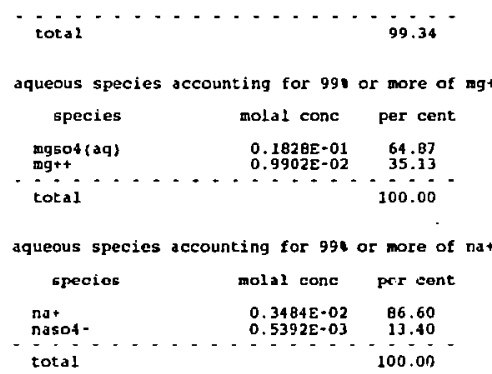

aqueous species accounting for 991 or hore of alo2(aq)

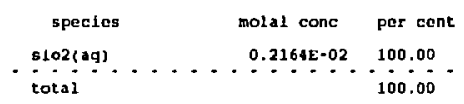

aqueous species accounting for 994 or nore of so4--

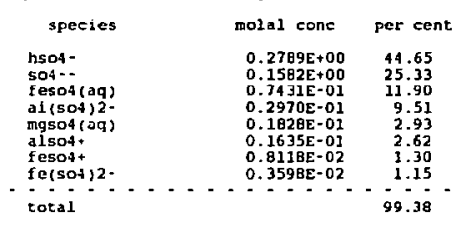

arjueous species dccounting for 998 or more of fet+

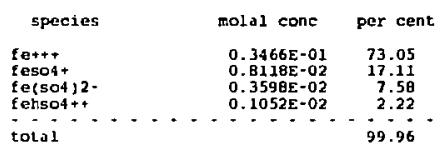

.... summary of aqueous redox reactions .....

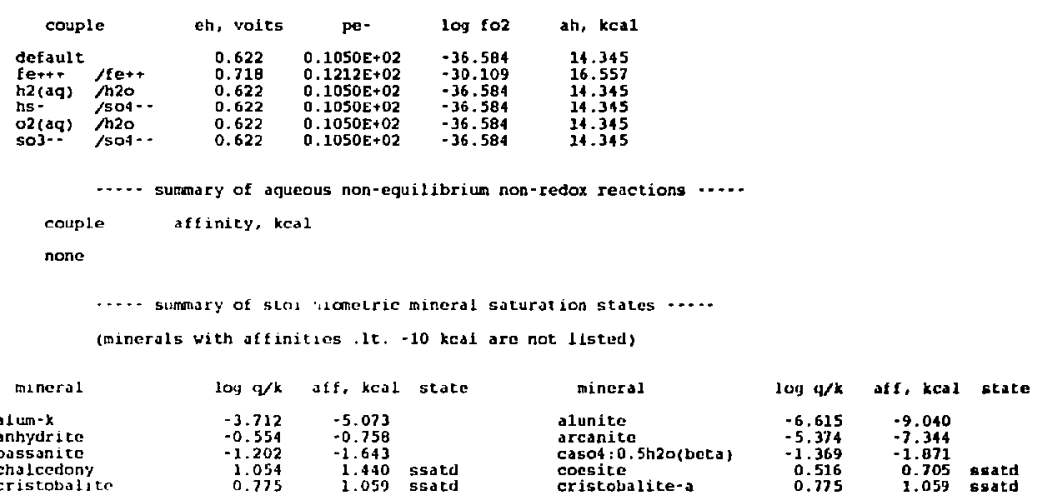




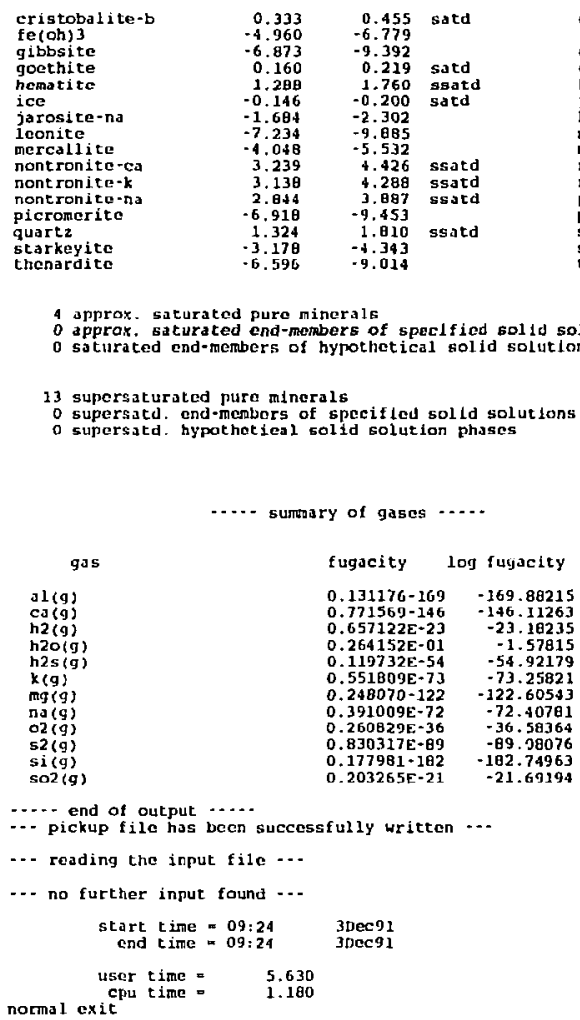

\subsection{The Dead Sea Brine Test Case}

Marcus (1977) attempted to calculate the activity of water and the mean activity of potassium chloride in Dead Sea brine. He was forced to use less accurate means than are presently available. Here, we repeat his work using Pitzer's equations and the hmw data file (based on Harvie, Møller, and Weare, 1984). As no meaningful $\mathrm{pH}$ measurement can be made for such a concentrated solution, we have here attempted to obtain an estimate of the $\mathrm{pH}$ and the $\mathrm{pHCl}$ function proposed by Knauss, Jackson, and Wolery (1990) by constraining the hydrogen ion to satisfy equilibrium with carbon dioxide in the atmosphere (log fugacity of $\mathrm{CO}_{2}=-3.5$ ). The dissolution reaction for $\mathrm{CO}_{2}$ gas can be written as:

$$
\mathrm{CO}_{2(g)}+\mathrm{H}_{2} \mathrm{O}_{(l)}=\mathrm{H}^{+}+\mathrm{HCO}_{3}^{-}
$$




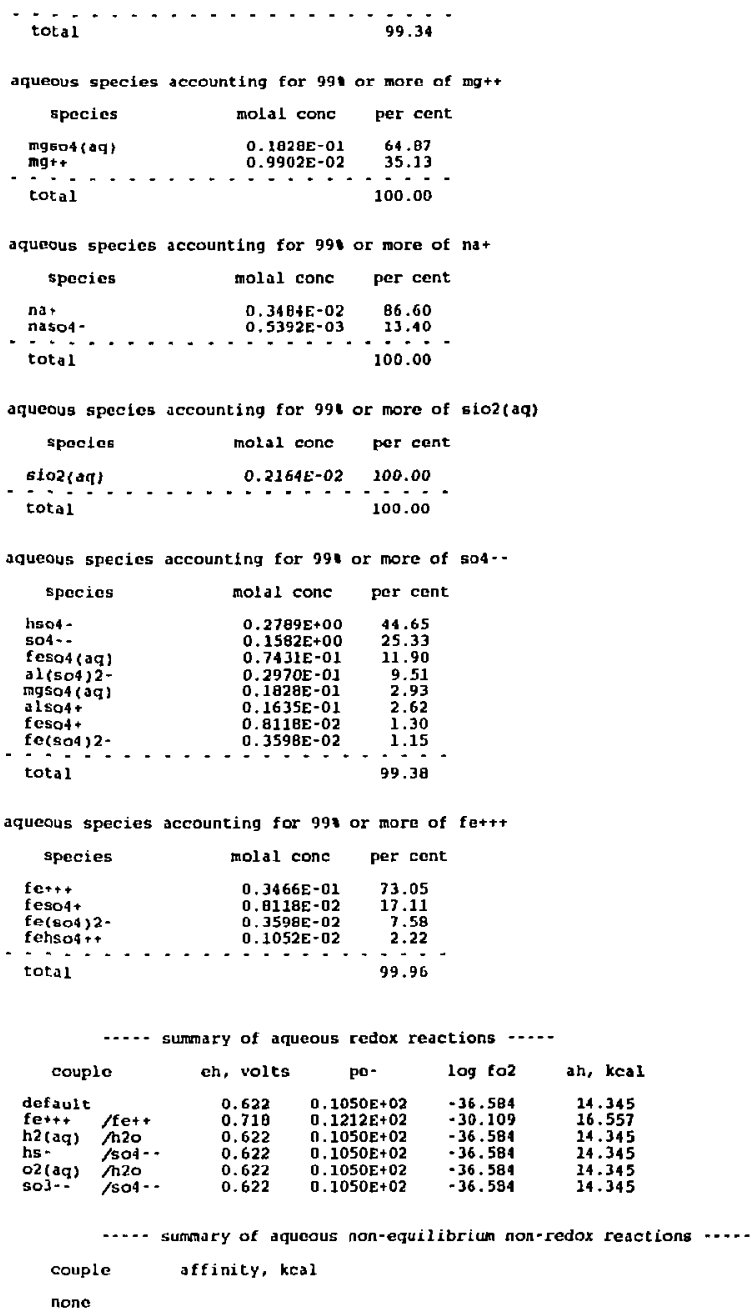




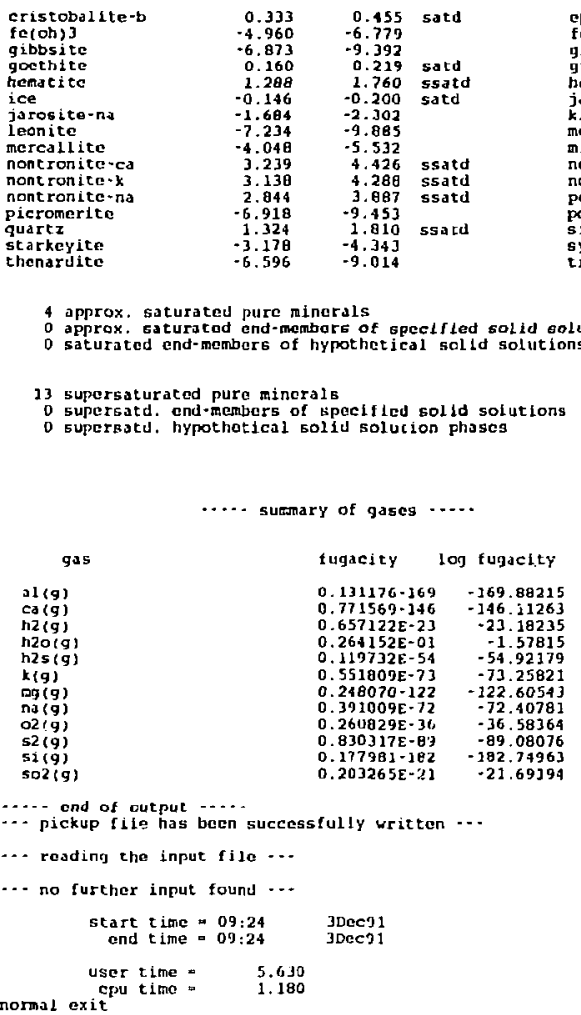

\subsection{The Dead Sea Brine Test Case}

Marcus (1977) attempted to calculate the activity of water and the mean activity of potassium chloride in Dead Sea brine. He was forced to use less accurate means than are presently available. Here, we repeat his work using Pitzer's equations and the hmw data file (based on Harvie, Møller, and Weare, 1984). As no meaningful $\mathrm{pH}$ measurement can be made for such a concentrated solution, we have here attempted to obtain an estimate of the $\mathrm{pH}$ and the $\mathrm{pHCl}$ function proposed by Knauss, Jackson, and Wolery (1990) by constraining the hydrogen ion to satisfy equilibrium with carbon dioxide in the atmosphere (log fugacity of $\mathrm{CO}_{2}=-3.5$ ). The dissolution reaction for $\mathrm{CO}_{2}$ gas can be written as:

$$
\mathrm{CO}_{2(g)}+\mathrm{H}_{2} \mathrm{O}_{(l)}=\mathrm{H}^{+}+\mathrm{HCO}_{3}^{-}
$$


The analytical data include a measurement of bicarbonate, which frees this reaction to be used as a constraint on the hydrogen ion. The input file is presented in both formats, and the output file is given starting with the message announcing the end of Newton-Raphson iteration.

Marcus (1977) concluded that the activity of water in Dead Sea brine was $0.754 \pm 0.004$. The EQ3NR calculation gives a value of 0.750 , in good agreement. Marcus also concluded that the mean ionic activity of $\mathrm{KCl}$ was in the range $0.876-1.199$. The value calculated by EQ3NR is somewhat lower, 0.827 . This is taken from the table of mean ionic properties, which was written on the output file because the option switch iopr6 was set to 1 on the input file. The $p H$ calculated by EQ3NR is 7.43 on the NBS scale and 8.50 on the scale on which $\log \gamma_{H^{+}}=0$. The $\mathrm{pHCl}$ is 6.94. Although the $p H$ of Dead Sea brine can not be measured in the usual way, it should be possible to measure the $\mathrm{pHCl}$ using the method proposed by Knauss, Jackson, and Wolery (1990).

Ben-Yaakov and Sass (1977) attempted to measure the $p H$ of artificial Dead Sea brine using a

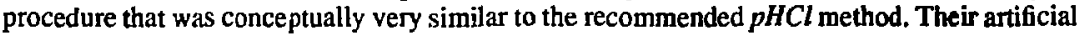
brine is very similar to that of Marcus (1977), but not identical. Using an electrochemical cell that in theory should respond linearly to $\mathrm{pHCl}$, they took emf measurements on the artificial brine and three $\mathrm{HCl}$ solutions. In order to obtain the $p H$ from their results, they had to estimate the activity coefficient of the chloride ion. They did this by first calculating the mean activity coefficient of potassium chloride in the brine using the Hamed rule (Hanned and Owen, 1958). This is an older approach to estimating activity coefficients in electrolyte mixtures which does not possess the accuracy of Pitzer's equations. They then estimated the activity coefficient of the chloride ion using the MacInnes (1919) convention:

$$
\gamma_{C l}=\gamma_{K^{+}}=\gamma_{ \pm, K C l}
$$

Using this approach, they determined that the $\mathrm{pH}$ of the artificial brine was 5.86. They compared this to the result of a conventional $p H$ measurement, which gave a value of 6.22. Their value of 5.86 certainly differs from the value of 7.43 that we obtained by assuming equilibrium with atmospheric carbon dioxide. What does this mean?

Ben-Yaakov and Sass (1977) obtained a value of 0.757 for the mean activity coefficient of $K C l$. The value obtained in our test (see the output file) is 0.823 . The corresponding values for the logarithm of this quantity are -0.1209 and -0.0846 , respectively. This means that their estimate of $\mathrm{pH}$ should be lower than ours by only 0.036 unit. A more significant problem is that Ben-Yaakov and Sass' use of the MacInnes convention has put their result on a "MacInnes" $p H$ scale. On the NBS scale used in our example, we obtained the following results:

$$
\begin{aligned}
& \underline{\text { Species }} \quad \underline{\log \gamma_{i}} \\
& K^{+} \quad 0.1055 \\
& \mathrm{Cl} \quad-0.2750 \\
& -0.0846 \text { (on the "Maclnnes" scale) } \\
& \text { Cl }-0.1209 \text { (Ben-Yaakov and Sass, 1977) }
\end{aligned}
$$


If we were to correct our result to the "MacInnes" scale, we would have to add $0.190 \mathrm{pH}$ unit to our result, which would give us a $\mathrm{pH}$ of 7.62. This moves us even farther away from agreement with Ben-Yaakov and Sass.

It seems most likely that the $p H$ of Dead Sea water must be closer to the value estimated by BenYaakov and Sass (1977). Therefore, the equilibrium fugacity of carbon dioxide must be higher than the atmospheric value used in our test case. Without conducting new measurements, the best approach to estimating the $\mathrm{pH}$ of Dead Sea brine is probably to update Ben-Yaakov and Sass' calculation using Pitzer's equations and expressing the results on the NBS scale. This can be done by finding the $\mathrm{pHCl}$ corresponding to Ben-Yaakov and Sass' emf measurements and using this as an input to EQ3NR (how to use $\mathrm{pHCl}$ as an input is demonstrated in the following example in this chapter). In order to find this $\mathrm{pHCl}$, one cotild use EQ3NR to calculate the $\mathrm{pHCl}$ of the three $\mathrm{HCl}$ solutions that Ben-Yaakov and Sass used as standards. One could then plot their measured emf results against these $\mathrm{pHCl}$ values. This plot could then be used as a calibration plot to determine the $\mathrm{pHCl}$ of the artificial brine from Ben-Yaakov and Sass' eñf measurement. The resulting calculation would give the equilibrium fugacity of carbon dioxide, which could be compared against the atmospheric value. This would be a good exercise for the user who is particularly interested in brine chemistry.

The EQ3NR input file (deadseabr.3i), the Dead Sea brine test case ("W" format):

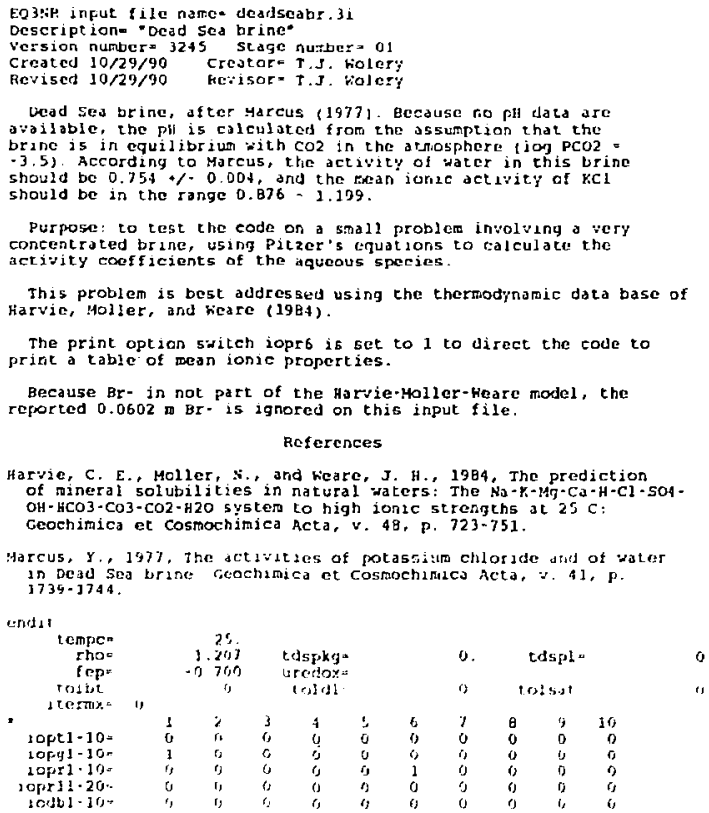




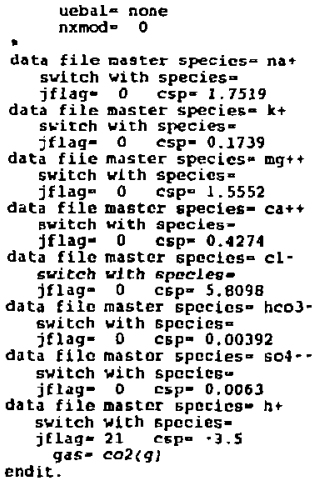

The EQ3NR input file (deadseabr,3i), the Dead Sea brine test case (" $D$ " format):

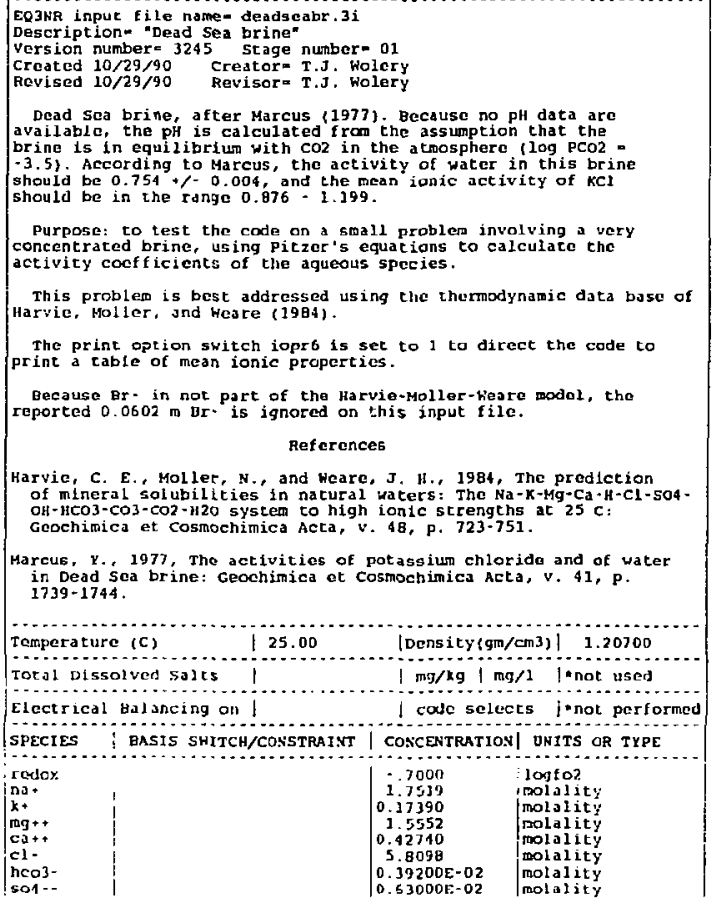

This problem is best addressed using the thermodynamic data boso of Harvie, Holler, und Heste (1984).

The print option switch iopro 1 s set to 1 to direct the code to print a table of mean ionic properties.

Because Br- in not part of the Harvie-Moller-heare modol, the

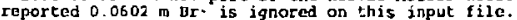

References

Harvie, C. E. Moller, N., and weare, J, H., 1984, The prediction of mineral solubilities in nacural waters: The Na-K-Mg-Ca+H-Cl-5O4OH-HCO3-CO3-CO2-H2O system to high ionle strengths at $25 \mathrm{C}$ : Geochimica et Cosmochimica Acta, v. 4B, P. 723-751.

marcus, $y ., 1977$, The activities of potassiun chlorida and of water in Dead Sea brine: Ceochimica et Cosmochimica Acta, v. 41, p. $1739+1744$.

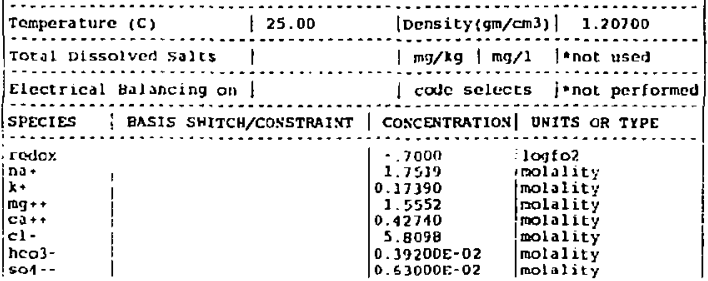




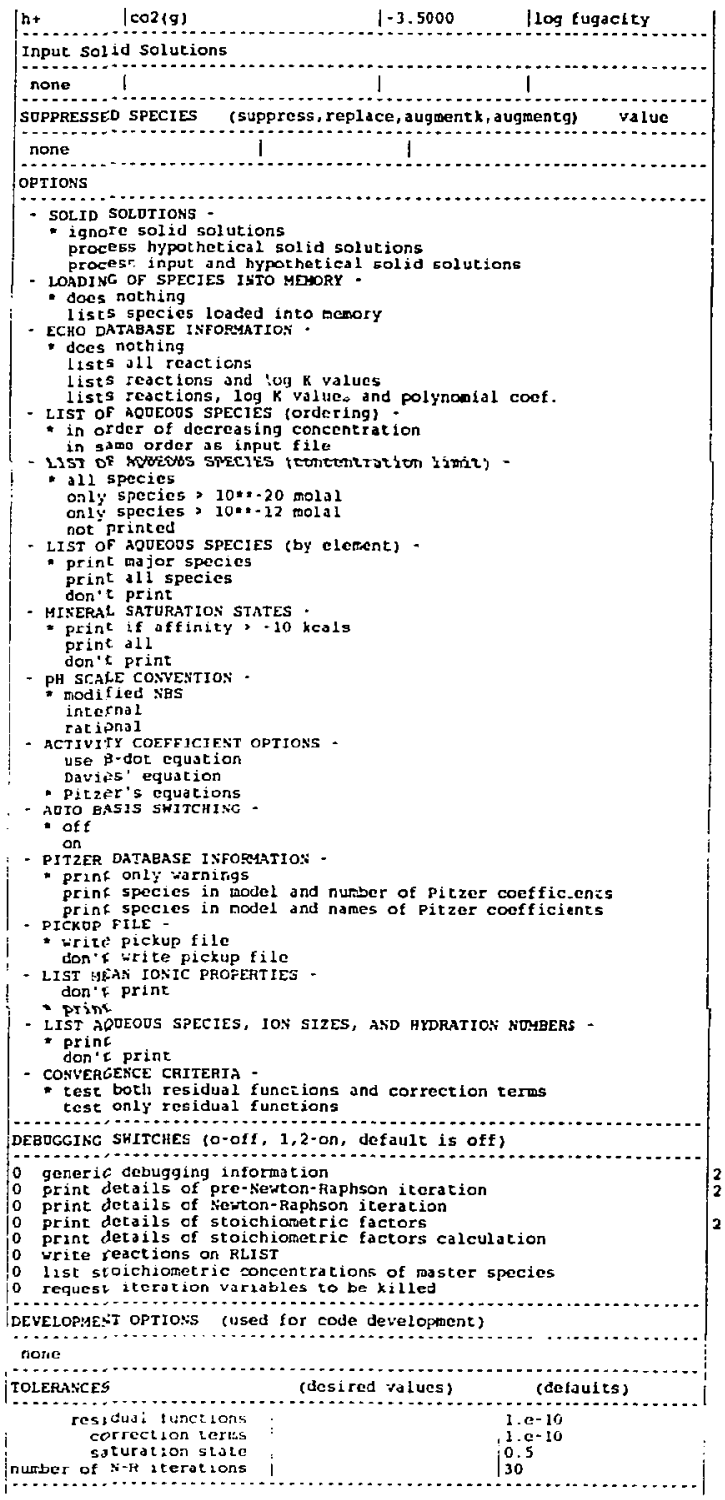




\section{The EQ3NR outnut file for the Dead Sea brine testcase (beginning with the message announcing} the end of Newton-Raphson iteration):

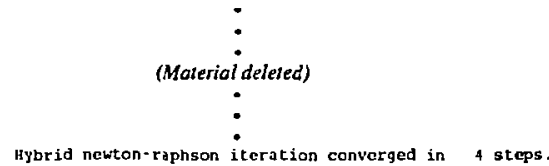

Summary of the Aqueous Phaso $\ldots . .$.

.... Elemental composition of the aqueous phase .....

element

$m g / 1$

$\mathrm{mg} / \mathrm{kg}$

moles/kg

$\begin{array}{ll}\text { ca } & 0,10727 E * 07 \\ \text { cl } & 20675 . \\ \text { h } & 0.24 B 61 E+06 \\ \text { c } & 0.13506 E * 06 \\ \text { h } & 56.829 \\ \text { ing } & 8206.6 \\ \text { ni } & 45624 . \\ \text { s } & 48613 . \\ & 243.8 j\end{array}$

0. 8887 OE +06

$$
\text { i7129. }
$$

$0.20597 \mathrm{E}+05$

0. 20597E+06

47.283

6799.2

37799

40276 .

202.02

$0.5554602070 E+02$

$0.4274000000 \mathrm{E}+00$

$0.5809 B 00000 \mathrm{E}+01$

$0.11101 \mathrm{BO} 110 \mathrm{E}+03$

$0.3920000001 \mathrm{E}+02$

$0.1739000000 \mathrm{E}+00$

$0.1555200000 E+01$

0.1751900000E+01

s

243.83

$0.6300000000 \mathrm{E}-02$

.... olemental composition as striet basis species .....

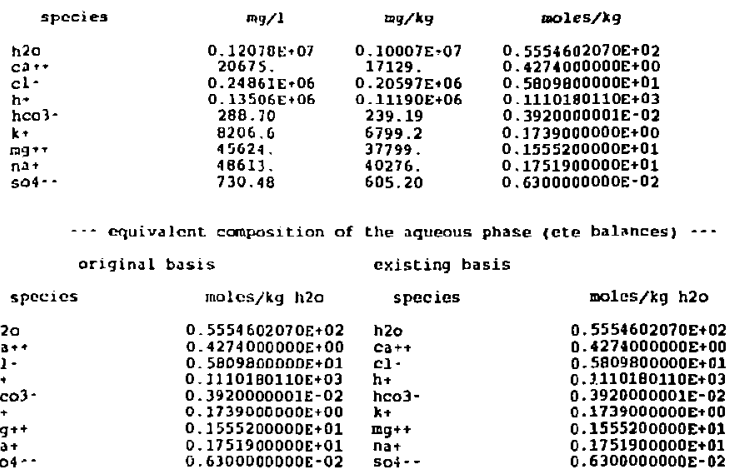

single ion setivities and activity coeficients are here defined with respect to the modified nbs ph scale

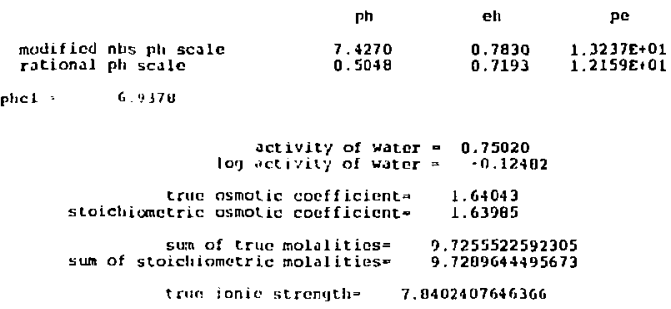


staichiometric ionic strength= 7.8486287476994

..... electrical balance totals ....

equiv/kg

$\begin{array}{rr}\text { sigma (mz) catlors }= & 0.5884630387 \mathrm{E}+01 \\ \text { sigma (mz) anions }= & -0.5823980803 \mathrm{E}+01 \\ \text { total charge }= & 0.117086119 \mathrm{E}+02 \\ \text { mean charge }= & 0.5854305595 \mathrm{E}+01 \\ \text { charge imbalance }- & 0.6064958353 \mathrm{E}-01\end{array}$

total charge - sigma (mz) cations + abs (sigma(mz) anions)

mean charge $=1 / 2$ cotal charge

the electriesl imbalance is

0.518
1.04
1.03
1.04

per cent of the total charge

per cent of the mean charge

per cont of signa (mx) cations

per cent of abs ( sfoma(mz) anions)

.... activity ratiog of lons -...
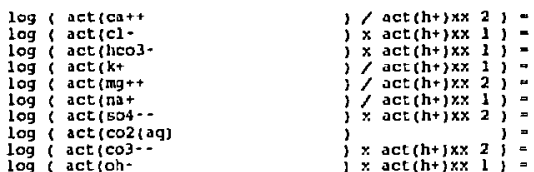

15.3905

-6.9378
.11 .4440

11.4440
6.7726

16.2165

B. 1563

$-19.7229$

$-4.9818$

-9.9818
-21.7633

log 6 act ioh-

$\{x \operatorname{act}(h+) x x \quad 1\}$

$+14.12 .15$

.... distribution of aqueous species ....

\begin{tabular}{|c|c|c|c|c|c|}
\hline species & molal cone & $\log \operatorname{con} c$ & $\log 9$ & jetivity & $\log$ act \\
\hline 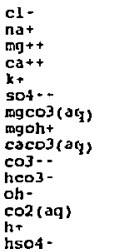 & $\begin{array}{l}0.5 B 10 E+01 \\
0.1752 E+01 \\
0.1552 E+01 \\
0.426 B E+00 \\
0.1339 E+00 \\
0.6300 E-02 \\
0.2259 E-02 \\
0.6245 E-03 \\
0.5735 E-03 \\
0.5322 E-03 \\
0.5115 E-03 \\
0.4793 E-05 \\
0.3702 E-05 \\
0.3120 E-0 B \\
0.795 B E-10\end{array}$ & $\begin{array}{r}0.7642 \\
0.2435 \\
0.1910 \\
-0.3697 \\
-0.7597 \\
-2.2007 \\
-2.6394 \\
-3.2044 \\
-3.2415 \\
-3.2739 \\
-3.2911 \\
-5.3194 \\
-5.4316 \\
-8.5048 \\
-10.0992\end{array}$ & $\begin{array}{r}-0.2750 \\
0.4858 \\
1.1716 \\
0.9063 \\
0.1055 \\
-2.6683 \\
0.0000 \\
0.0599 \\
0.0000 \\
-3.6555 \\
-0.7259 \\
-1.3751 \\
0.1498 \\
1.0778 \\
-0.2102\end{array}$ & $\begin{array}{l}0.3084 \mathrm{E}+01 \\
0.5362 \mathrm{E}+01 \\
0.2304 \mathrm{E}+02 \\
0.3440 \mathrm{E}+01 \\
0.2217 \mathrm{E}+00 \\
0.1352 \mathrm{E}-04 \\
0.2299 \mathrm{E}-02 \\
0.7169 \mathrm{E}-03 \\
0.5735 \mathrm{E}-03 \\
0.1177 \mathrm{E}-06 \\
0.9615 \mathrm{E}-04 \\
0.2020 \mathrm{E}-06 \\
0.1043 \mathrm{E}-04 \\
0.3741 \mathrm{E}-07 \\
0.4816 \mathrm{E}-10\end{array}$ & $\begin{array}{r}0.4692 \\
0.7293 \\
1.3625 \\
0.5366 \\
-0.6542 \\
-4.8690 \\
-2.6394 \\
-3.1445 \\
-3.2415 \\
-6.9294 \\
-4.0170 \\
-6.6945 \\
-4.9610 \\
-7.4270 \\
-10.3173\end{array}$ \\
\hline
\end{tabular}

.... tpeas ianic properties .....

true (a)

In $(+/-) \quad g(+/-)$

2. $433 \mathrm{E}+00$

1. $315 \mathrm{E}+00$

$\begin{array}{ll}\text { 4. } 116 \mathrm{E}-03 & 6.579 \mathrm{E}-01 \\ 5.166 \mathrm{E}-02 & 1.315 \mathrm{E}-01\end{array}$

1. 507E-02

2. $140 \mathrm{E}-04$

1.34BE-04

1. 265E-06

3. 950E-07

1. $005 \mathrm{E}+00$

$1.005 E+00$
$9.431 E-03$

9. $431 E-03$
5. $754 E-02$

5. 7 SAE-02

$2.525 \mathrm{E}=02$
$9.130 \mathrm{E}=04$

9. $130 \mathrm{E}=04$
$3.742 \mathrm{E}+0$

$7.406 \mathrm{E}-0.3$

2. 689E-02

2. 074 E-02

3. 29$] \mathrm{E} \cdot 0$.

3. $190 \mathrm{E}+00$

2. $94.4 \mathrm{E}-02$

2. $684 \mathrm{E}-01$

1.178E-01
4.221E-02

2. $429 \mathrm{E}-01$

1. $500 E+00$

$6.747 \mathrm{E} \cdot 0$

3. $163 \mathrm{E}-0$

B. $227 \mathrm{E}-01$

4. $895 \mathrm{E}-01$

1. $517 \mathrm{E}-0$

2. 318E-0.

1. $611 E+00$

8. $065 \mathrm{E}-0$

$5.728 \mathrm{E}-02$

$5.728 \mathrm{E}-02$

?. $977 \mathrm{E}-01$

1. $.255 E+00$
$7.585 \mathrm{E}-01$

2. $719 E-0$ ]

1. 275E-0]

3. $592 \mathrm{E}-0$ ]
7. 109E-02 stoichionet ric (b)

$m(+/-)$

$9(+/-)$

2. $434 \mathrm{E}+00 \quad 1.314 \mathrm{E}+00$

1. B73E-02 1.692E-01

5. I69E-02 $1.314 \mathrm{E} \cdot 01$

B. $452 \mathrm{E}-02$ G. $150 \mathrm{E}-0$

1.796E+01 $1.892 \mathrm{E}-05$

4.665E-01 4.066E-06

2. $688 \mathrm{BE}+00$ 9.916E-0B

1.005E+00 8.227E-0

2.611E-02 1.768E-01

5.75AE-02 1.517E-01

0.085E-02 2.610E-03

3.744E-00 1.6J0E+00 2. $300 \mathrm{E}-02$ 2.073E-01

$9.898 E-02 \quad 1.793 E-01$

1.300E-01 7.537E-04

3.10OE+0O 1.235E+00

$8.2 B 7 E-02 \quad 2.740 E \cdot 01$

\begin{tabular}{ll}
$2.684 \mathrm{E}-01$ & $2.740 \mathrm{I} \cdot 01$ \\
\hline
\end{tabular}

2. $560 \mathrm{E} \cdot 01 \quad 4.056 \mathrm{E} \cdot 03$

(a) true guantitios consistent rith the speriation model

(b) stoichiometric quantitics consistent fith the cle mass 
batance lumpings, except that

1. ef Eective ete(h+) - cte(h+) - conc (hzo)

2. effective cte(oh-) a cte(h2o) - conc(h2o)

.... major aquoous species contributing to mass balanses -...

aqueous species accounting for 991 or thore of cat+

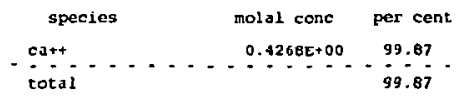

aqueous species accounting for g9t or more of cl-

spectes molal conc per cent

c1. . . . $0.5810 \mathrm{z}+01 \quad 100.00$

total 100.00

aqueous specles accounting for g9s or more of hool-

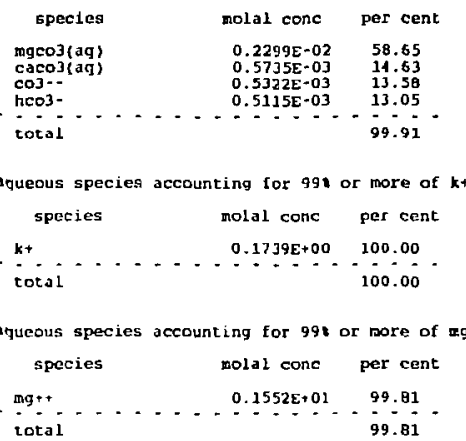

atqueous sfw cies accounting for 991 or more of nat

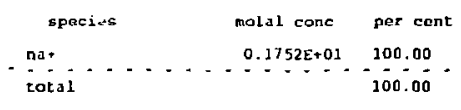

aqueous species accounting for 991 or more of so4--

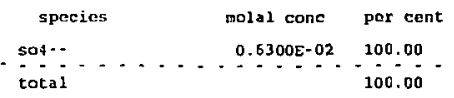

-... sunnary of aqueous redox reactions -...

$\begin{array}{ccccc}\text { couple } & \text { ch, volts } & \text { PE } & \log \text { fo2 } & \text { ah, kcal } \\ \text { Jefult } & 0.783 & \text { 0.1324E }+02 & -0.700 & 18.059\end{array}$

.... sumnary of aqueous non-equilibrium non-redox reactions ....

couple affinity. keal

none

-... summary of staichimetric ciencral saturation stotes ....

(mererals Fith affinities. Lt. - 10 keal are not listed, 


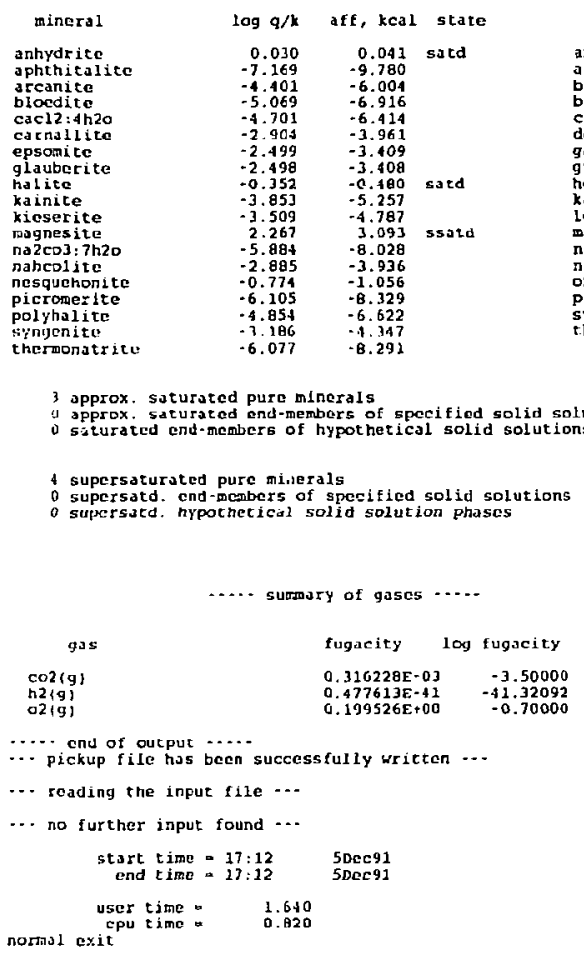

\subsection{Using $\mathrm{pHCl}$ as an Input: An Example}

In the earlier example treating sea water with Pitzer's equations, we found that the $\mathrm{pHCl}$ was computed to be 8.6722 . The $p H$ value that was used as an input in this example was 8.22 on the NBS scale. The purpose of the present example is to show how $\mathrm{pHCl}$ can be used in place of $\mathrm{pH}$. Here we partially invert the earlier problem by entering the $\mathrm{pHCl}$. We should then get back the original $p H$. Other results (i.e., the calculated electrical imbalance, values of the mineral saturation indices) should also match the results from the previous example. This problem was run using the same hmw data file. The input file is presented in both formats. The output file is given starting with the message announcing the end of Newton-Raphson iteration.

The computed $p H$ on the NBS scale is printed on the output file as " 8.2200 ." The correctness of the inversion is therefore verified. One may compare other computed results to verify that the computed model is otherwise identical to that obtained previously. 
The EQ3NR input file (swphcl.3i), the $p H C l$ test case ("W" format):

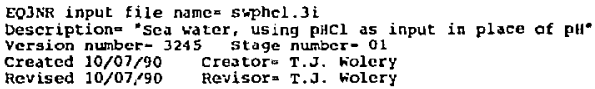

Sea water, including only the mujor ions. This is a considerably pared-down version of swtst. $3 i$, which contains the full benchmark sea water test case of Nordstrom et al. (1979, Tuble III). This input flle is a variant of simajp.inf, which uscs Pitzer's equations. Here the input for pH $(\theta .22$ ) has been repluced by one for pHcl $(8.6717)$, the value obtained Js output $\mathrm{E}$ om running swmajp. 31 and using the thermodynamic Jata base of Iarvic, Molles, and weare \{1984\}. The output of running the present input file should include $\mathrm{pH}$ of Q. 22 .

Purpose; to test the pHcl-typa (loq activity combination\} input option.

Feferences

Harvic, C. E., Moller, N., and Weare, J. H., 1984, The prodlet lon of mineral solubilities in nacural wacers: The Na-K-Hg-Ca-H-Cl-5O4-

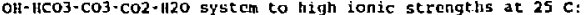
Geochimica et Cosmochimice Acts, v. 48, p. 723-751.

Nordstram, D. K., et a 1 , 1979, A comparisan of computerized cheraical models for equilibrium calculations in aqueous systems, in Jenne, E. A.. editor, Chemical Modeling in Aqueous systens, ACs Symposium Series, v. 93, Mncricsn Cliemicol society, washington, D.C..
p. $357-892$.

endit.

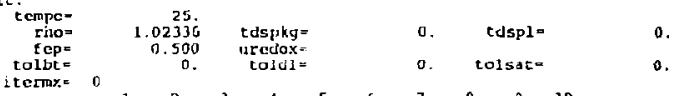

\begin{tabular}{|c|c|c|c|}
\hline 1 & 2 & 3 & 4 \\
\hline$-\overrightarrow{1}$ & 0 & 0 & 0 \\
\hline 1 & 0 & 0 & 0 \\
\hline 0 & 0 & 0 & 0 \\
\hline D & 0 & D & 0 \\
\hline 0 & D & 0 & 0 \\
\hline
\end{tabular}

iopr $1-70$

iop $11-30=$

ic. $\mathrm{bl} \cdot 1 \mathrm{Dc}$

uebal= none

nxpod= 0

to file moter species= no,

switeh with species =

jflago 3 cspr 10760 .

data filc daster species $k$.

switch wich species\%
jflag 3 cspo 34,1

cata file moster species cat switeh with species=

jflage 3 cspe 412.3

data file waster species $\mathrm{mgt}^{+}$

switch vith speciesm

jflog: 3 c5p=1291.

data file master speciesw h*

switcli with spocies=

jflag= 17 ssp=-6.6722

ion= 01 -

data file master species: heo3-

switch with species"

jflog= 0 esp .002022

dota filc moster species= clswitch with species

switch with speciesf
jflag 3 cspo 19353 .

data file master spccies so4 -

switch with species:

endit.

jlage 3 aspa 2712 .

The EQ3NR innut file (swohcl.3i), the $\mathrm{pHCl}$ test case ("W" format):

EOJNR input file nange sinplacl

Descriptione "Sea iater. Usini rilfel as anput in place of pll"

version number $=3275$ stajo number = 01

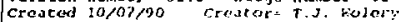

Reviser 10\%O?/90 [erisor T.J. haler'; 


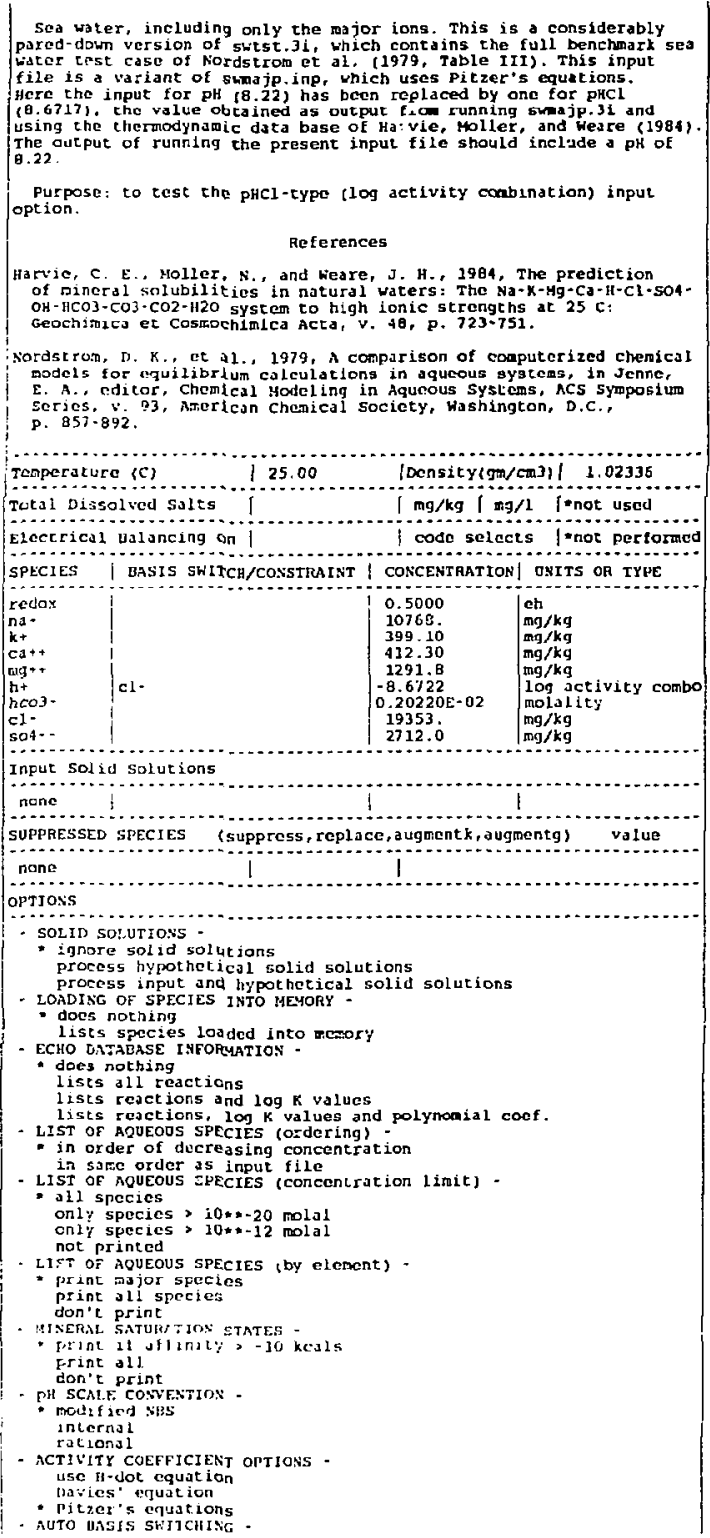




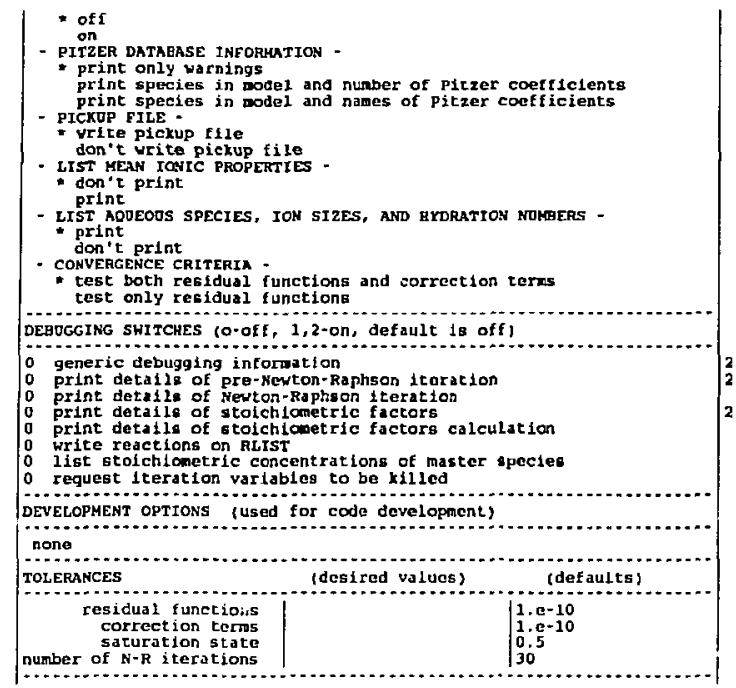

The EQ3NR output file (swohcl.30), the $\mathrm{pHCl}$ cest case (beginning with the message announcing the end of Newton-Raphson iteration):

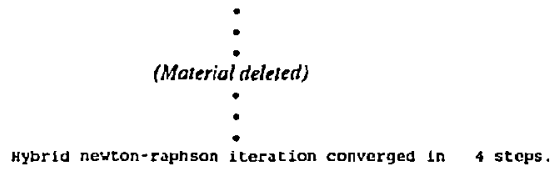

Hybrid newton-raphson iteration converged in 4 steps.

- Sumary of the aqueous Phase...-

Elemental composition of the aqueous phaso

\begin{tabular}{|c|c|c|c|}
\hline element & $\mathrm{mg} / \mathrm{l}$ & $\mathrm{mg} / \mathrm{kg}$ & moles/kg \\
\hline $\begin{array}{l}0 \\
\text { ca } \\
c 1 \\
h \\
c \\
\mathbf{h} \\
\text { mg } \\
\text { na } \\
\text { s }\end{array}$ & $\begin{array}{l}0.91080 E+06 \\
421.93 \\
19805 \\
0.11451 E+06 \\
24.854 \\
408.42 \\
1322.0 \\
11020 \\
926.41\end{array}$ & $\begin{array}{l}0.89001 \mathrm{E}+06 \\
412.30 \\
19353 . \\
0.11190 \mathrm{E}+06 \\
24.286 \\
399.10 \\
1291.8 \\
1076 \mathrm{~B} \\
905.26\end{array}$ & $\begin{array}{l}0.5552742040 E+02 \\
0.1028743949 E-01 \\
0.5458822600 E+00 \\
0.1110186924 E+03 \\
0.2022000000 E-02 \\
0.1020760493 E-01 \\
0.5314955770 E-01 \\
0.4683822413 E+00 \\
0.2823129677 E-01\end{array}$ \\
\hline
\end{tabular}

... elemental composition as strict basis species ....

\begin{tabular}{|c|c|c|c|}
\hline species & $\pi 0 / 1$ & $\mathrm{mg} / \mathrm{kg}$ & $\operatorname{moles} / \mathrm{kg}$ \\
\hline $\begin{array}{l}\text { h2o } \\
\text { cat+ } \\
\text { cl- } \\
\text { h+ } \\
\text { heol. }\end{array}$ & $\begin{array}{l}0.10256 E+07 \\
421.93 \\
19805 \\
0.11451 E+06 \\
126.26\end{array}$ & $\begin{array}{l}0.10021 E+07 \\
412.30 \\
19353 \\
0.11390 E+06 \\
123.30\end{array}$ & $\begin{array}{l}0.5562742040 \mathrm{E}+\mathrm{D2} \\
0.1028743949 \mathrm{E}+\mathrm{DI} \\
0.5458822600 \mathrm{E}+00 \\
0.11101 \mathrm{B6924E}+03 \\
0.2022000000 \mathrm{E}-02\end{array}$ \\
\hline
\end{tabular}




$\begin{array}{llll}\mathrm{k+} & 408.42 & 399.10 & 0.1020760493 E-01 \\ \mathrm{ng}++ & 1322.0 & 1291.8 & 0.531495570 E-01 \\ \mathrm{nat} & 11020 . & 10768 . & 0.468382213 E+00 \\ \text { sot- } & 2775.4 & 2712.0 & 0.2823129677 \mathrm{E}-01\end{array}$

- equivalent composition of the aqueous phase (cte balanced) -.. original basie

existing bag1s

\begin{tabular}{|c|c|c|c|}
\hline spectes & moles/kg h2o & spacies & moles/kg h2o \\
\hline $\begin{array}{l}\text { h2o } \\
\mathrm{cat}+ \\
\mathrm{cl}- \\
\mathrm{ht} \\
\text { heol= } \\
\mathrm{xt} \\
\text { ngt+ } \\
\text { nat } \\
\text { sot-. }\end{array}$ & $\begin{array}{l}0.5562742040 E+02 \\
0.102874394 E-01 \\
0.5458922600 E+00 \\
0.1110186924 E+03 \\
0.2022000000 E-02 \\
0.2020760493 E-01 \\
0.5314955770 E-01 \\
0.4683822413 E+00 \\
0.2823129677 E-01\end{array}$ & $\begin{array}{l}\text { h2o } \\
\text { cat+ } \\
\text { cl- } \\
\text { ht } \\
\text { heoj- } \\
\text { k+ } \\
\text { ng+t } \\
\text { nd+ } \\
\text { got. }\end{array}$ & $\begin{array}{l}0.5562742040 E+02 \\
0.1028743949 E-01 \\
0.5458622600 E+00 \\
0.1110186924 E+03 \\
0.2022000000 E-02 \\
0.1020760493 E-01 \\
0.5314955770 E-01 \\
0.4683922413 E+00 \\
0.2823129677 E-01\end{array}$ \\
\hline
\end{tabular}

alngle ton activities and activity cooffleionte aro horo defined

with respect to the modleded nos ph veaid

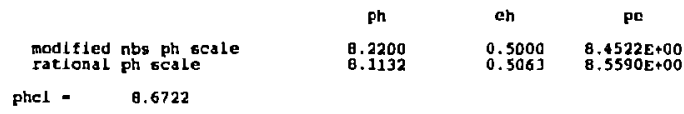

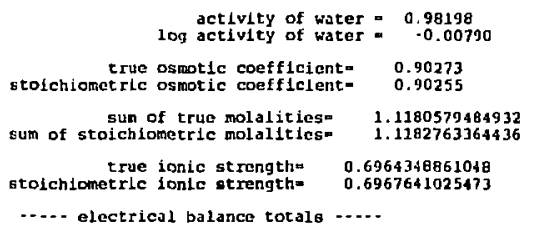

equiv/kg

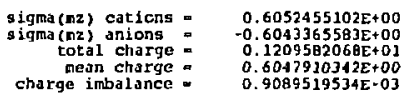

total charge = sigma(mz) cations + abs (sigma(mz) anions)

mean charge * 1/2 total charge

the electrical imbalance is

0.751E-01 per cent of the total charge

0.150 per cent of the mean charge

0.150 per cent of sigma(nz) cationg

0.150 per cent of abs (algma(mz) anions)

.... activity ratios of lons .....

\begin{tabular}{|c|c|c|c|c|c|c|}
\hline $\begin{array}{l}\log ! \\
\log ! \\
\log ! \\
\log ! \\
\log ! \\
\log ! \\
\log ! \\
\log ! \\
\log ! \\
\log !\end{array}$ & 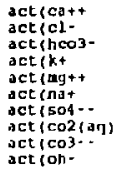 & $\left\{\begin{array}{l}x \\
x \\
\vdots \\
\vdots \\
x \\
x \\
x\end{array}\right.$ & $\begin{array}{l}\operatorname{act}(h+) \times x \\
\operatorname{act}(h+) \times x \\
\operatorname{act}(h+) x x \\
\operatorname{act}(h+) x x \\
\operatorname{act}(h+) \times x \\
\operatorname{act}(h+) x x \\
\operatorname{act}(h+) x x \\
\operatorname{act}(h+) x) \\
\operatorname{act}(h+) x x\end{array}$ & $\left.\begin{array}{l}2 \\
1 \\
1 \\
1 \\
2 \\
1 \\
2 \\
2 \\
2 \\
1\end{array}\right\}$ & $\begin{array}{l}: \\
: \\
: \\
: \\
: \\
:\end{array}$ & $\begin{array}{r}13.7845 \\
-8.6722 \\
-11.2062 \\
6.0292 \\
11.5350 \\
7.7243 \\
-19.0097 \\
-4.8609 \\
-21.5455 \\
-24.0046\end{array}$ \\
\hline
\end{tabular}

... distritution of aqueous specios ....

\begin{tabular}{|c|c|c|c|c|c|}
\hline species & molal conc & $\log$ conc & $\operatorname{lo}\} g$ & jctlvity & log act \\
\hline $\begin{array}{l}\text { c1: } \\
\text { na* } \\
\text { mg": } \\
\text { soi:- }\end{array}$ & $\begin{array}{l}0.5459 \mathrm{E}+00 \\
0.4604 \mathrm{E}+00 \\
0.5306 \mathrm{E}-01 \\
0.2823 \mathrm{E}-01\end{array}$ & $\begin{array}{l}-0.2629 \\
-0.3294 \\
-1.2752 \\
-1.5493\end{array}$ & $\begin{array}{l}-0.1093 \\
-0.1663 \\
-0.6291 \\
-1.0204\end{array}$ & $\begin{array}{l}0.3530 E+00 \\
0.3194 E+00 \\
0.1247 E-01 \\
0.2694 E-02\end{array}$ & $\begin{array}{l}-0.4522 \\
-0.4957 \\
-1.9043 \\
-2.5696\end{array}$ \\
\hline
\end{tabular}




\begin{tabular}{|c|c|c|c|c|c|}
\hline 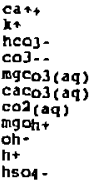 & $\begin{array}{l}0.1026 \mathrm{E}-01 \\
0.1021 \mathrm{E}-01 \\
0.1816 \mathrm{E}-02 \\
0.8641 \mathrm{E}-04 \\
0.8292 \mathrm{E}-04 \\
0.2456 \mathrm{E}-04 \\
0.1224 \mathrm{E}-04 \\
0.3362 \mathrm{E}-05 \\
0.3029 \mathrm{E}-05 \\
0.7706 \mathrm{E}-08 \\
0.2332 \mathrm{E}-08\end{array}$ & $\begin{array}{l}-1.9887 \\
-1.9911 \\
-2.7409 \\
-4.0634 \\
-4.0813 \\
-4.6097 \\
-4.9121 \\
-5.4734 \\
-5.5187 \\
-6.1132 \\
-6.6322\end{array}$ & $\begin{array}{r}-0.6668 \\
-0.1998 \\
-0.2452 \\
-1.0420 \\
0.0000 \\
0.0000 \\
0.0512 \\
-0.0279 \\
-0.2658 \\
-0.1068 \\
-0.1788\end{array}$ & $\begin{array}{l}0.2210 E-02 \\
0.6444 E-02 \\
0.1032 E-02 \\
0.7844 E=05 \\
0.6292 E-04 \\
0.2456 E-04 \\
0.1376 E=04 \\
0.3152 E=05 \\
0.1642 E=05 \\
0.6025 E-00 \\
0.1545 E=08\end{array}$ & $\begin{array}{l}-2.6555 \\
-2.1909 \\
-2.9662 \\
-5.1054 \\
-4.0013 \\
-4.6097 \\
-4.0609 \\
-5.5014 \\
-5.7846 \\
-6.2200 \\
-0.0111\end{array}$ \\
\hline
\end{tabular}

... major aqueous species contributing to mass balances -...-

aqueous species accounting for 99 or more of catt

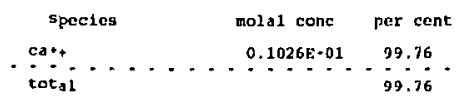

aqueous species accounting for 99 or more of $\mathrm{cl}$.

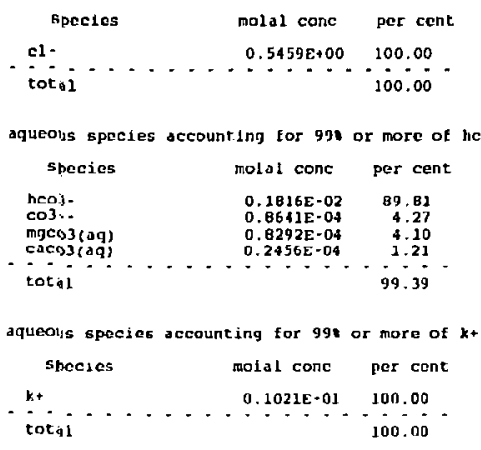

aqueous species accounting for 992 or more of mg+t

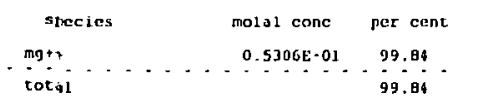

aqueous species accounting $f \circ 5991$ or more of hat

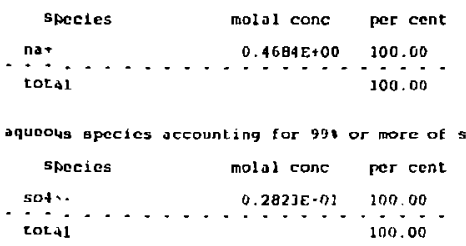

\begin{tabular}{|c|c|c|c|c|}
\hline couple & cil, volls & pe- & lou Jo? & $a h, k \in a l$ \\
\hline tef a & 0.500 & $0.8452 \mathrm{E}+01$ & -16.432 & 11.531 \\
\hline
\end{tabular}

.... sumarary of arguedots non-eruilibrium non-redor reactions ....

couplo jotinity, xeal. 
(minerals with affinities. 1t. - 10 kcal are not listed)

mLneral

antiydrite

arcanitc

bloedite

calctio

glubuer 1

halle

kalnitg.

mirablite

natca (sot) 3 : 2h20

natron

oxychloridc-m

piessonito

oyngenlte

thermonatito $\log q / x$

aff. xeal state

$-0.663$

$-5.175$

$-5.720$

0.645

$-2.640$

$-3.542$

$-2.518$

$-6.948$

.4 .359

$-2.412$

$-6.691$

$-5.351$

$-5.686$

$-4.630$

$-4.736$

$-6.587$
$-1.177$

$-7.060$

$-7.803$

0.860 sgatd

$-3.61$

$-4.832$

$-3.436$

-3.436
-9.479

$-5.947$

$-3.291$

$-9.126$

$-7.300$

$-7.757$

$-6,317$

$-6.461$

$-0.986$ minera i

$\log q / x$

aragonite

bischof $1 \mathrm{t}$

brucite

dolomite

gaylussite

gypsu

hexahydrito

kalicinite

magnesite

na2cos:7h2o

nahcolite

nesquehonite

picronerito

Byluito

thonardito
0.459

$-7.311$

$-2.589$

2. 312

2.312
-4.476

$-0.660$

$-2.8 B 6$

$-5.450$

0,024

$-5,692$

$-3,079$

-1.866

$-7,145$

$-3.543$

$-3.274$
Jff, keal seate

0.626 seatd

.9 .975

$-3.532$

3.154 ssatd

.6 .107

$-0.901$

$-3.938$

$-7.44$

1.124
-7.265

$-4.200$

$-2.546$

$-9.746$

$-4.834$

.4 .466

o approx. saturated pure ininerals

0 approx. suturated end-nembers of specif 1 ed solid solutions

0 saturoted end-members of hypothetical solid solutions

4 supersaturated pure ninerals

0 supersatd. ond-rocmbers of specified bolid enlutions

o supersatd. hypothecieal solid solution phases

.... sumary of gases $\ldots . .$.

gas fugacity log fugacity

$\begin{array}{llr}\operatorname{col}(g) & 0.417753 E-03 & -3.37908 \\ h 2(g) & 0.459200 \mathrm{E}-3] & -3] .33800\end{array}$

$0.369030 E-16 \quad-16.43200$

-... end of output -....

.. pickup file has been successfully written ...

-. - reoding the input file ...

... no further input found ...

$$
\text { start time }=11: 11 \text { 17Dec91 }
$$

end $t$ ime $=11: 12$ 170ecs

normal exit

user time - $\quad 1.580$

cpu time - 1.060




\section{The EQ3NR to EQ6 Connection: The Pickup File}

EQ3NR creates a model of an aqueous solution. The EQ6 code may then be used to calculate models of geochemical processes in which this solution is an initial component. The two codes are constructed so that the initial model constraints on the aqueous solution are input only to EQ3NR. The user does not repeat this information in the user-created portion of the EQ6 input file. Instead, EQ3NR writes the necessary data on a small file called pickup.

EQ6 normally reads the information from the EQ3NR pickup file as the bottom part of the EQ6 input file. It can also read it directly under an option that can be specified on the EQ6 input file. The contents of the EQ3NR pickup file will be discussed in more detail in the EQ6 Theoretical Manual and User's Guide (Wolery and Daveler, 1992b). EQ3NR automatically writes a pickup file unless the user specifies otherwise (by setting iopt $3=-1$ ).

The example given in this chapter is the pickup file written for the sea water example presented in Chapter 7. This is given in both "W" and "D" formats; the code writes the pickup file in the format corresponding to the input file used. Discussion here will focus primarily on the example in "W" format.

The following discussion assumes that the reader is familiar with the parameters that appear on the EQ3NR input file (Chapter 6). The pickup file begins with a repetition of the original title (utitl). This is useful because it carries any documentation identifying the particular aqueous solution model.

The following line contains the temperature $\left({ }^{\circ} \mathrm{C}\right)$ of the EQ3NR run, which is specified in the variable tempci. The EQ6 run that uses this input may actually start at a different temperature. If so, there is said to be a temperature jump. The tempci input allows EQ6 to recognize when this is the case and to issue a warning to the user.

The alter/suppress options follow in the same format as on the EQ3NR input file. In this example, no such options are specified, hence nxmod $=0$. If any had been specified, two lines would have followed for each option, one for the uxmod parameter and another for the jxmod, kxmod, and xlkmod parameters.

The next five inputs (kct, kmt, kxt, kdim, and kprs) are key parameters in the scheme by which EQ6 organizes important variables. Here ket is the number of chemical elements in the system. The variable kdim is the number of total master species, which in EQ6 includes not only the aqueous master species discussed in this report, but also any mineral species in equilibrium with the aqueous solution. The variables $\mathbf{k m t}$ and $\mathbf{k x t}$ mark, respectively, the last pure mineral and last solid-solution end-member that are in the master species set. These species must have corresponding masses present in the geochernical model. This is never the case in EQ3NR, and consequently no such species can appear among the master spacies written on an EQ3NR pickup file. This condition is marked by $\mathbf{k m t}$ and $\mathbf{k x t}$ having values equal to that of $\mathbf{k d i m}$. The variable kprs is the number of pure mineral and solid-solution end-member species in the "physically removed system," a concept relating only to the flow-through open system model in EQ6. Hence, kprs must always be zero on the EQ3NR pickup file. 
Following the parameters discussed above are the symbols of the kct chemical elements in the system and their masses in moles relative to one kilogram of solvent (mte). The calculated electrical imbalance (electr) is given in the same format.

The last part of the EQ3NR pickup file gives the names of the "run" master iteration variables (the "run" basis species), the corresponding "data file" basis species, and the values of the corresponding variables. These are the base ten logarithms of the molal concentrations of the "run" basis species. If a "run" basis species is not the same as the corresponding "data file" master species, it marks the pair of species for a basis switch.

\section{Example of an EO3NR pickun file in "W" format:}

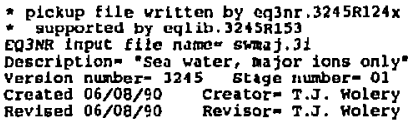

sea water, including only the major ions. This is a considerably pared-down version of swtst. 31, which contalns the $f$ ul 1 benchmark sea water test case of Nordstrom et al. (1979, Table III).

Purpose: to test the code on a small problem involving a moderately concentrated solution. The activity coefficients of the aqueous species are calculated from the $B$-dot equation and related equations.

References

Nordstrom, D. K., et al., 1979, A comparison of computerized cherical models for equilibriun calculations in aqueous systems, in Jenne, E. A., editor, Chemical wodeling in Aqueous Systems, Acs symposium series, Y. 93, American Cherical society, washington, D.C.. p. $857-892$.

endit.

Cempci $=0.25000 E+02$

nxmod-

iopg1=

iopg4 - 0

iopg7. 0

$10 p g 10=0$

kct $=9$

$\circ$

$k \times t=10$

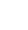

iopg2= 0

iopg6= 0

ksq= 10

opg9= 0

KMt $=10$

o. $556274103683972 \mathrm{E}+02$

kprs* 0

ca

$0.102874395765819 \mathrm{E}-01$
$0.545882260556139 \mathrm{E}+00$

$0.545882260556139 \mathrm{E}+00$

$0.202200001512726 \mathrm{E}-02$

$0.102026049472423 \mathrm{E}-01$

D. $531495584904613 E-01$

0. $531495584904613 \mathrm{E}-01$

$0.282312979158034 \mathrm{E}-01$

0.954079658003204 E- 03

h20

electr

h2o

$\mathrm{Cat+}$

cl.

cat+

cl-

ht

heo3- heos-

mg+t

nat

$504=$

kt

mg+

nat--

02 ( 9 )

$0.174435898252698 E+0$

$-0.203301523866778 E+01$

$-0.280373596842018 E+00$

$-0.810794436314582 E+01$

- 0 2892436344923985401

$0.200002837042004 E 01$

-

$-0.1390173513623758+0$

-0.187880994095832540

026

$-0.164317709391211 E 402$ 


\section{Example of an EQ3NR pickup file in "D" format:}

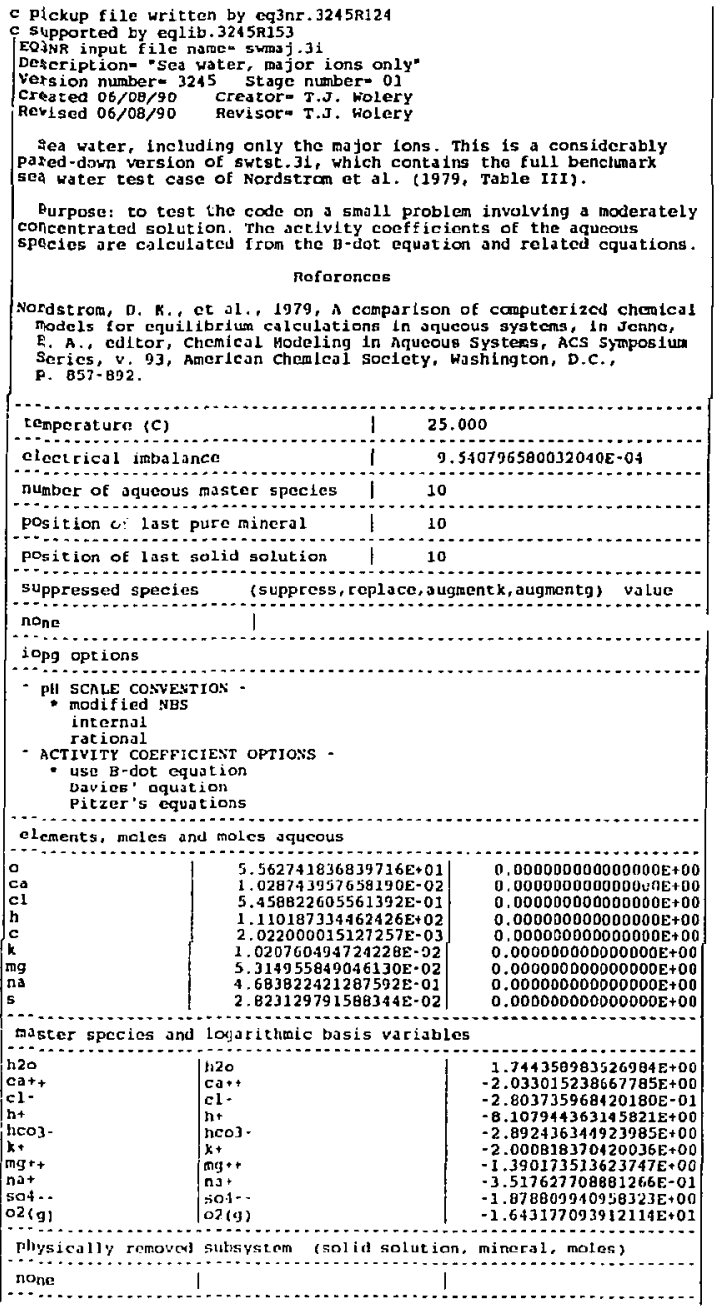




\section{Solving the Governing Equations}

\subsection{Introduction}

The governing equations that apply to speciation-solubility modeling were introduced in Chapters 2,3 , and 4 . The purpose of this chapter is to derive the mathematics necessary to solve them. The approach is to set up the problem in terms of $n$ equations in $n$ unknowns (or "iteration variables") and solve them. Technically, there are a large number of equations and corresponding unknowns to deal with. The unknowns include the concentrations of the all the species appearing in the model and their thermodynamic activity coefficients. The comesponding equations are algebraic, and these must be solved using appropriate methods.

In EQ3NR, the set of unknowns is first reduced to a relatively small set of unknowns, from which the remaining unknowns can be calculated. These are the primary iteration variables. They are defined in this code as the log concentrations of the species in the active basis set. The algebraic equations are solved by a combination of two iterative methods which are applied in sequence. The first method, called pre-Newton-Raphson optimization, has the characteristic of rapid convergence far from the solution, and slow (limiting first order) convergence near the solution. It is used primarily to get all of the primary iteration variables within an order of magnitude of the solution. The second method, a hybrid Newton-Raphson method, has the characteristic of poor convergence behavior far from the solution, and very fast (limiting second order) convergence near the solution. These methods thus complement one another. We will discuss these, as well as supplementary methods designed to aid convergence. Lastly, we will briefly discuss the subject of crash diagnostics.

\subsection{The Set of Master Iteration Variables}

In the EQ3NR code, the number of equations and unknowns is reduced by substituting all governing aqueous mass action equations into the mass balance and electrical balance equations. The remaining aqueous species giving rise to unknowns then comprise the relatively small active basis set. These master iteration variables teside in the vector 3 , which has the following structure:

$$
z=\left[\begin{array}{c}
\log x_{w} \\
\cdot \\
\cdot \\
\log m_{s^{\prime}}, s^{\prime}=1, s_{B}-1, s \neq w \\
\cdot \\
\log f_{O_{2}} \\
\cdot \\
\cdot \\
\log m_{s^{\prime}}, s^{\prime}=s_{B}+1, s_{Q}
\end{array}\right]
$$


The first part of this vector contains entries for the $s_{B}$ strict basis species appearing in a given problem. The second part contains any auxiliary basis variables which appear in the problem and for which $\mathbf{j f l a g} \neq 30$ (those with jflag $=30$ are treated as non-basis species). The structure is further simplified if $s_{Q}=s_{B}$ (no active auxiliary basis set). For a problem in which this is the case, it is implied that the aqueous solution is in a state of complete internal (homogeneous) equilibri$u m$. If $s_{Q}=s_{B}+1$, complete internal equilibrium is also implied if the $s_{Q}$ th species is part of a redox couple used to define the redox state of the fluid (the ioptl $=1$ option). In all other cases, partial internal disequilibrium is implied.

We will first look at the aqueous mass action equations that are to be eliminated. Here $s^{\prime \prime}$ denotes a non-basis species and $r$ denotes the corresponding reaction. As index labels, these are related according to:

$$
r=s^{\prime \prime}-s_{B}
$$

This reflects the fact that strict basis species appear first in the list of all aqueous species and that they have no associated reactions. A mass action equations takes the following logarithmic form:

$$
\begin{gathered}
\log K_{r}=b_{w r}\left(\log x_{w}+\log \lambda_{w}\right)+b_{s_{A} r} \log f_{O_{2}}+b_{s^{\prime \prime} r}\left(\log m_{s^{\prime \prime}}+\log \gamma_{s^{\prime \prime}}\right) \\
+\sum_{\substack{s^{\prime}=1 \\
s^{\prime} \neq w, s_{B}}}^{s_{Q}} b_{s^{\prime} r}\left(\log m_{s^{\prime}}+\log \gamma_{s^{\prime}}\right)
\end{gathered}
$$

where $K_{r}$ is the thermodynamic equilibrium constant for the reaction $b_{s r}$ is the reaction coeffcient for the $s$-th species, $\lambda_{w}$ is the activity coefficient of water, and $\gamma_{s}(s \neq w)$ is the molal activity coefficient of the $s$-th species. Note that $s^{\prime}$ implies a basis species, and that $s^{\prime \prime}$ denotes the only non-basis species appearing in the reaction. This equation can be rearranged to give:

$$
\begin{gathered}
\log m_{s^{\prime \prime}}=\frac{\log K_{r}}{b_{s^{\prime \prime} r}}-\log \gamma_{s^{\prime \prime}}-\frac{b_{w r}}{b_{s^{\prime \prime} r}}\left(\log x_{w}+\log \lambda_{w}\right)-\frac{b_{s_{B} r}}{b_{s^{\prime \prime} r}} \log f_{O_{2}} \\
-\sum_{\substack{s^{\prime}=1 \\
s^{\prime} \neq w, s_{B}}}^{s_{Q}} \frac{b_{s^{\prime} r}}{b_{s^{\prime \prime} r}}\left(\log m_{s^{\prime}}+\log \gamma_{s^{\prime}}\right)
\end{gathered}
$$

Recall the relation:

$$
\frac{d x}{d \log x}=2.303 x
$$

It follows that: 


$$
\frac{d m_{s^{\prime \prime}}}{d x}=2.303 m_{s^{\prime \prime}} \frac{d \log m_{s^{\prime \prime}}}{d x}
$$

Treating the activity coefficients as constants, this can be used to show that:

$$
\begin{aligned}
& \frac{\partial m_{s^{\prime \prime}}}{\partial \log x_{w}}=-2.303 m_{s^{n}} \frac{b_{w r}}{b_{s^{\prime \prime} r}} \\
& \frac{\partial m_{s^{\prime \prime}}}{\partial \log f_{D_{2}}}=-2.303 m_{s^{n}} \frac{b_{s_{B} r}}{b_{s^{\prime \prime} r}} \\
& \frac{\partial m_{s^{\prime \prime}}}{\partial \log m_{s^{\prime}}}=-2.303 m_{s^{\prime \prime}} \frac{b_{s^{\prime} r}}{b_{s^{\prime \prime} r}}, \quad s^{\prime}=1, s_{Q}, s^{\prime} \neq w, s_{B}
\end{aligned}
$$

These relations will be used later in this chapter in deriving the Jacobian matrix elements corresponding to the mass and charge balance residual functions. This matrix is used in Newton-Raphson iteration.

Certain factors will appear repeatedly in some of the derivations below and will be given special symbols. In the EQ3NR code, these parameters are themselves evaluated before the calculation of the Jacobian matrix elements in which they appear. This is done to avoid repetitive arithmetic in the code. These are defined as follows:

$$
\begin{gathered}
H_{s^{\prime} r}=\frac{m_{s^{\prime \prime}} u_{s^{\prime \prime} s^{\prime}}}{b_{s^{\prime} r}}, s^{\prime}=1, s_{Q}, s^{\prime} \neq w \\
H_{z r}=\frac{m_{s^{\prime \prime}} z_{s^{\prime \prime}}}{b_{s^{\prime \prime} r}}
\end{gathered}
$$

All of the so-called alternative constraints involve equations which are written only in terms of the concentrations (or activities) of species in the active basis set. Therefore, it is not necessary to make any substitutions of the sort noted above in dealing with the mass balance equations and the charge balance equation.

\subsection{Expanding the System from the Set of Master Iteration Variables}

If one knows the vector $\underline{z}$, one may "expand the system" by computing the concentrations of all non-basis species (all species not in the active basis set; this includes any auxiliary basis species with jflag values of 30 ) and the activity coefficients of all species in solution. The $\underline{z}$ vector uniquely defines all system properties. However, the process of expanding the system is not exactly straightforward. In order to calculate the concentrations of the non-basis species, one must evaluate the corresponding mass action equations. The activity coefficients appear in these equations. Hence, the activity coefficients must be evaluated first. However, the activity coefficients depend in general on the concentrations of all solute species, both basis and non-basis. So to deal with these, one must compute the concentrations of the non-basis species first. 
This creates a problem analogous to the old puzzle, "Which came first, the chicken or the egg?" In previous versions of EQ3/6 (e.g., Wolery, 1983), this problem was overcome by treating the ionic strength as a master iteration variable. The equation defining the ionic strength has the same form as a mass balance equation, and this equation was treated in like form. However, this only works if the activity coefficient model depends only on the ionic strength, not the specific composition of the solution. Thus, this approach works for the Davies equation and the B-dot equation, but not for Pitzer's equations or any other set of equations likely to be valid in concentrated solutions.

The concentrations of non-basis species may vary over many orders of magnitude. The activity coefficients of aqueous species gerierally vary over about two orders of magnitude or less. The approach taken in EQ3/6 is to start by computing a set of reasonable values for the activity coefficients, then hold these constant until the concentrations of the non-basis species become reasonably stable. The activity coefficients are then updated. In the pre-Newton-Raphson optimization algorithm, the computed concentrations of the basis and non-basis species are typjcally adjusted 3-7 times before the activity coefficients are recalculated. In the hybrid NewtonRaphson method, they are recalculated between each Newton-Raphson step (it is because of this treatment that we refer to our usage of the Newton-Raphson method as a hybrid). In either case, the system is expanded by first calculating the new concentrations of the non-basis species, using the existing values of the activity coefficients. The activity coefficients are then recalculated. An exception to this order occurs when starting values are constructed (see below).

One could view the expansion itself as an iterative process. One could recalculate the concentrutions of the non-basis species, recalculate the activity coefficients, and then repeat the process o.1e or more times. In the context of the pre-Newton-Raphson optimization method, this makes li tle sense because this method is only used to get in the neighborhood of the solution. The merits of a single update, a double update, and a multiple update (repeating the process until a convergence tolerance is satisfied) were examined in the context of the hybrid Newton-Raphson methc .. Significant differences in performance were only observed in the case of highly concentrat 1 electrolyte solutions. Thus, in the case of less concentrated solutions, the single update me ،od was best because it gave the same performance for the lowest cost. In the case of the more concentrated solutions, it was found that the single update also gave the best performance, fo'lowed by the multiple update method. In such solutions, the double update method often led to failure to converge. The method which has been adopted, therefore, is the single update method.

\subsection{Beginning the Process: Computing Starting Values}

The whole process must begin by assigning starting values. This is done in mod le arrset.f. Initially, this is done as follows. For every basis species with a jflag value of less than 15 , either the total or free molality is known. The concentration of each such individuai basis species is assigned this corresponding value. This value is an upper bound if the corresponding concentration is the total molality, and the actual value if it is a free molality. For species 1 aving a jflag value of 16 , the $\log$ accivity is known. The concentration of each such species is assigned a value equal to the thermodynamic activity, thus assuming that the activity coefficient has unit value. All other basis species are initially assigned a value of $1 \times 10^{-7}$ molal. All non-basis species at this point have assigned concentrations of zero. 
The charge imbalance is computed. The furctions $\Sigma m$ and the ionic strength are then estimated. The estimate of the ionic strength at this point includes a term in which the computed charge imbalance is treated as though it were due to an unmeasured monovalent ion. The mole fraction of water is computed from the value of $\Sigma m$. Then the activity coefficients are computed. The concentrations of non-basis species are taken as zero until the code enters the pre-Newton-Raphson optimization stage.

Assuming that the concentration of a basis species is equal to its total concentration may or may not be a good approximation. If it turns out to be a very poor approximation, then the first estimate of the concentration of at least one non-basis species will have to be large, typically a few to a few tens of orders of magnitude greater than the limit on its actual concentration imposed by the corresponding mass balance constraint. This first estimate is very often quite large in an absolute as well as a relative sense, often on the order of $10^{+10}$ to $10^{+60}$ molal. It is critical not to compute functions such $\Sigma m$, the ionic strength, and the activity coefficients until the concentrations for such species have been brought down to physically realistic values.

\subsection{Methods to Aid Convergence}

Several techniques are used in EQ3NR to aid convergence, both in pre-Newton-Raphson optimization and hybrid Newton-Raphson iteration. These are:

- Use of logarithmic iteration variables.

- Under relaxation techniques.

- Automatic and user-specified basis switching.

We have not found it necessary to employ other methods, such as the "curve-crawler" technique discussed by Crerar (1975).

The physical quantities that correspond to the iteration variables are intrinsically positive. Use of logarithmic iteration variables restricts the generated values to the physically reasonable range. Also, logarithmic corrections are effectively relative corrections to $t$. orresponding physical quantities. Recall that $d \log x / d x=1 /(2.303 x)$. It follows that:

$$
\Delta \log x \approx \frac{\Delta x}{2.303 x}
$$

Because of this, effective under-relaxation techniques are especially easy to implement when using logarithmic iteration variables.

Under-relaxation is the technique of judiciously reducing the magnitude of the computed correction terms. Assume that the unmodified method involves adding a correction term vector $\left(\delta_{k}\right)$, where $k$ is the iteration number. This is typical in Newton-Raphson iteration. The new vector of master iteration variables is obtained thusly:

$$
z_{k+1}=z_{k}+\underline{\delta}_{k}
$$


If the new vector of master iteration variables is obtained instead by evaluating some set of corresponding equations not in this format, one can still utilize under-relaxation by defining a correction term vector as follows:

$$
\underline{\delta}_{k}=z_{k+1}-z_{k}
$$

Global under-relaxation is effected by replacing the correction equation given above by:

$$
z_{i+1}=z_{i}+\kappa \delta_{i}
$$

where $\kappa$ is a positive number less than one. Non-global under-relaxation is also possible. This does not involve the use of an under-relaxation factor. Rather it involves truncating the magnitudes of individual correction terms to satisfy specified limits, which may be different depending on the species involved and on the direction of change.

There are several methods of applying global under-relaxation. ${ }^{2} Q^{3} ! R$ uses two relatively simple ones in making Newton-Raphson steps. The first of these places a limit on the element of the correction term vector having the largest magnitude:

$$
\kappa=\frac{\delta^{\prime}}{\delta_{\max }}
$$

where $\delta^{\prime}$ is the imposed limit and $\delta_{\max }$ is the max norm of $\delta$. In a Newton-Raphson iteration step (which occurs in the EQLIB module nrstep.f), this imposed limit is represente $\mathrm{A}$ by the variable screwd. In EQ3NR, this is set in the main program (module eq3nr.f) at a value of 2.0 . Besides aiding convergence, this method causes divergenee, when it does occur, to occur more slowly. In such cases, it helps to yield useful information aovut the cause of divergence.

The other global under-relaxation method is applied for only the first 8 iterations. The under-relaxation factor is cut in half if the residual vector max norm $\beta_{\text {mux }}$ exceeds the value of the variable screwn. Initially, $\kappa$ is set to a value of unity; when the current method of under-relaxation is applied, this factor may have been reduced as a result of applying the method described above. In EQ3NR, screwr. is set to 0.5 in the main program (module eq3nr.f).

Some degree of non-global under-relaxation is also employed in pre-Newton-Raphson optimization. This optimization function takes place in EQ3NR in module arrset.f. Here uncier-relaxation is effected by imposing truncation limits on changes for individual master variables. The master variables for species constrained by mass balance equations are not permitted to decrease by more than $20.0 \log$ units in a given step. A master variable constrained by the charge balance equation may not change by more than $2.0 \mathrm{log}$ units.

Some truncation limits also apply to the activity coefficients and the functions $\Sigma m$ and the ionic strength. These limits are applied during both pre-Newton-Raphson optimization and hybrid Newton-Raphson iteration. These limits are defined in the variable chgfac, which is in the calling sequence of the EQLIB module ngcadv.f. The value of this variable is set in the calling modules, and is usually scaled inversely with the value of $\Sigma \mathrm{m}$. Values range from 1.3 to 100. 
If automatic basis switching is turned on (iopt2 = 1), EQ3NR (in the module arrset.f) will attempt to improve the starting values by means of basis switching. The methodology here is quite simple. Consider the case of dissolved aluminum. The data file basis species is $A l^{3+}$. At iow temperature, typically low values of dissolved aluminum, and moderate to high $p H$, the mass balance is typically very strongly dominated by the species $\mathrm{AllOH})_{4}^{-}$. The concentration of $\mathrm{Al}^{3+}$ is many orders of magnitude below the concentration of this species. If one assumes that the concentration of this species is instead essentially equal to the total concentration, the computed concentration of $\mathrm{Al}(\mathrm{OH})_{4}^{-*}$ may be something on the order of $10^{20}-10^{40}$ molal. The value of the corresponding residual function will be similarly extremely large. On the other hand, when $\mathrm{Al}(\mathrm{OH})_{4}{ }_{4}$ is in the basis set, the initial assumption is that its concentration is equal to the total concentration, and the computed concentration of $\mathrm{Al}^{3+}$ is an appropriately much smaller number.

In the present version of EQ3NR, the total concentration quantity associated with a mass balance is redefined in terms of the new basis species. Assuming this quantity is expressed as molality, this change has no numerical significance in the above example. However, if a species to be switched into the basis set contributes to the original total concentration by a factor different from that of the original basis species, the difference is quite significant. For example, if $\mathrm{Al}_{13} \mathrm{O}_{4}(\mathrm{OH})_{24}^{7+}$ is switched into the basis set in place of $\mathrm{Al}^{3+}$, then the associated total concentration must be redefined as:

$$
m_{T, A l_{13} O_{4}(O H)_{24}^{7+}}=\frac{m_{T, A l^{3+}}}{13}
$$

Automatic basis switching is accomplished in a loop structure. More than one switch may be done each time through the loop. After this, the activity coefficjents are recomputed (again assuming that the concentrations of non-basis species are zero), the residual functions are recomputed and more switches may be made. In the process, some switches may be undone by later ones. For example, $\mathrm{Al}_{13} \mathrm{O}_{4}(\mathrm{OH})_{24}^{7+}$ may first be switched into the basis set in place of $\mathrm{Al}^{3+}$, and then $\mathrm{Al}(\mathrm{OH})_{4}{ }^{-}$in place of, $\mathrm{Al}_{13} \mathrm{O}_{4}(\mathrm{OH})_{24}^{7+}$. When one switch replaces another, the original switch is first undone. In this example, $A l^{3+}$ is switched back into the basis in place of $\mathrm{Al}_{13} \mathrm{O}_{4}(\mathrm{OH})_{24}^{7+} ; \mathrm{Al}(\mathrm{OH})_{4}$ is then switched into the basis in place of $\mathrm{Al}^{3+}$. This loop continues until there are no candidates for basis switching or the loop has been passed through nlopmx times. This variable is currently set to 12 in a data statement in arrset.f.

To be a candidate for automatic basis switching, a species must have a computed concentration ten times that of the corresponding basis species. Furthermore, it can not already be in the basis set. A data file basis species which has been switched out of the basis set can only be brought back into the basis set by undoing an earlier switch. The involvement of a basis species in the input constraint associated with another basis species may prevent switching it out of the basis set. For example, if the input constraint for $\mathrm{H}^{+}$is a value for the $\mathrm{pHCl}$ function, then $\mathrm{Cl}$ is locked into the basis set. Conflicts may arise in candidate basis switches. For example, the same species 
could dominate more than one mass balance. It is then switched into the basis so as to reduce the affected mass balance residual which has the highest value.

The user may specify certain basis switches on the input file (see Chapter 5). These switches are executed prior to entry to module arrset.f and the construction of the initial starting estimates. If certain switches really need to be made, it is more efficient to make them in this manner. If the software is run on a machine with a small floating point exponent range, such as a VAX system lacking the G_FLOATING option, the code may stop execution because of a floating point overflow (in the computation of one of the elements of the residual function vector $\beta$ ) unless such switches are directed on the input file. In such a situation, one may have to guess which switches are necessary to avoid such overflow.

If automatic basis switching is turned off (jopt2 $=0$ ), the code will proceed directly from having made the initial starting estimates into pre-Newton-Raphson optimization. If it is tumed on and the user has directed certain switches so be made on the input file, it may undo one or more of those switches, as well as make additional switches.

\subsection{The Pre-Newton-Raphson Optimization Algorithm}

After any automatic basis switching is completed, arrset.f uses an optimization algorithm. This process occurs in a loop structure, the times through which are known as passes. At the end of a pass, the activity coefficients are recomputed. If the concentration of an ion is to be adjusted to satisfy electrical balance, this adjustment is also recomputed at this point. Within each pass is another loop structure, the times through which are called cycles. Here, adjustments are made to the concentrations of the basis species (other than one which is constrained to satisfy electrical balance), A pass is completed after some number of cycles. The cycles within a pass terminate if some rather rough convergence criteria are satisfied, or if the maximum number of cycles in a pass have been completed. This is determined by the variable ncylim, which is currently set to 15 in a data statement in arrset.f. The passes terminate if rough convergence criteria applying to both the cycles and passes are satisfied, or if the maximum number of passes has been completed. This is determined by the variable nplim, which is currently set to 7 in a data statement in arrset.f.

The cycle algorithm is applied only to basis species which are constrained by mass balances. It is an example of what is sometimes called a "continued fraction" method. A variation on this approach (Wolery and Walters, 1975) was in fact the principal method used to solve speciation-solubility problems in a an early version of what is now EQ3NR. The derivation to be given here is different than that previously given elsewhere, and includes an important modification that has apparently not been previously noted.

Consider the case of dissolved aluminum. The total dissolved aluminum is expressed as total $A l^{3+}$. The normalized mass balance residual is:

$$
\beta_{A l^{3+}}=\frac{m_{T, c a l c, A l^{3+}-m_{T, A l^{3+}}}}{m_{T, A l^{3+}}}
$$


where: $m_{T, \text { calc, } A l^{3+}}$ is the total concentration of $A l^{3+}$ as calculated from a mass balance expression, using the current estimated values of the concentrations of the basis species and estimates of the concentrations of non-basis species as calculated from the associated mass action equations, usi $\mathrm{g}$ in these the current estimated values of the concentrations of the basis species. In contrast, $m_{T, A l^{3+}}$ is one of the model constraints.

We will assume, for the moment, that $A l^{\beta+}$ dominates this calculated mass balance. We may express this by writing;

$$
m_{A l^{3+}} \approx m_{T, \text { calc, } A l^{3+}}
$$

We may then write:

$$
\beta_{A l^{3+}} \approx \frac{m_{A l^{3+}}-m_{T, A l^{3+}}}{m_{T, A l^{3+}}}
$$

We can rearrange this to:

$$
\frac{m_{A l^{3+}}}{\beta_{A l^{3+}}+1} \approx m_{T, A l^{3+}}
$$

We take the current iteration in the cycle to be the $k$-th. Applying the above equation to this iteration, we may write:

$$
\frac{m_{A l^{3+}, k}}{\beta_{A l^{3+}, k}+1} \approx m_{T, A l^{3+}}
$$

Similar, applying it to the next iteration (the $k+1-$ th) gives:

$$
\frac{m_{A l^{3+}, k+1}}{\beta_{A l^{3+}, k+1}+1} \approx m T, A l^{3+}
$$

Combining these equations then gives:

$$
m_{A l^{3+}, k+1} \approx \frac{m_{A l^{3+}, k}\left(\beta_{A l^{3+}, k+1}+1\right)}{\beta_{A l^{3+}, k}+1}
$$

We would like the residual function to approach zero quickly. This desire can be written as: 


$$
\boldsymbol{\beta}_{A l^{3+}, k+1} \approx 0
$$

Substitution of this into the above equation gives the following the iteration equation:

$$
m_{A l^{3+}, k+1}=\frac{m_{A l^{3+}, k}}{\beta_{A l^{3+}, k}+1}
$$

In a dilute, acid solution, the species $A l^{3+}$ will indeed dominate its own mass balance. The starting assumption based on this should be a good one. Also, this species should dominate the calculated mass balances during the iteration process. We would expect the above iteration equation to work well. However, in solutions of moderate to high $\mathrm{pH}$, the species $\mathrm{Al}(\mathrm{OH})_{4}{ }^{-}$dominates the mass balance of $A l^{3+}$. What happens then? Note that we could do a basis switch, replacing $A l^{3+}$ with $A l(O H\rangle_{4}$. The aboye eg̣uations would then apply to $A l(O H)_{4}{ }^{-}$and all should again work well.

If we do not make this basis s witch, we would still assume that $\mathrm{Al}(\mathrm{OH})_{4}^{-}$dominates the calculated mass balances. Following the previous approach, we are led to an equation of the form:

$$
m_{\mathrm{Al}(\mathrm{OH})_{4}^{-}, k+1}=\frac{m_{\mathrm{Al}(\mathrm{OH})_{4}^{;}, k}}{\beta_{\mathrm{Al}^{3+}, k}+1}
$$

This is almost what we would have if we had made the basis switch. However, in this case, the normalized residual is still defined in terms of $\mathrm{Al}^{3+}$, not $\mathrm{Al}(\mathrm{OH})_{4}^{-}$. However, ve can not directly use this equation, because it is written in terms of molalities of $A l(O H)_{4}$, not $\mathrm{Al}^{3+}$. However, we can conver it into a form in terms of molalities of $\mathrm{Al}^{3+}$. The two species are related by the reaction:

$$
\mathrm{Al}(\mathrm{OH})_{4}^{-}+4 \mathrm{H}^{+}=\mathrm{Al}^{3+}+4 \mathrm{H}_{2} \mathrm{O}_{(l)}
$$

The corresponding mass action equation is:

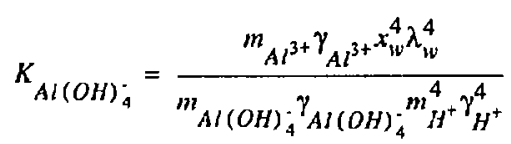

Let us consider all activity coefficients to be fixed, as well as the concentrations of other basis species appearing in this relation. Then the concentrations of the two aluminum species of interest satisfy the following proportionality:

$$
m_{A l^{3+}} \propto m_{A l(\mathrm{OH})_{4}}
$$


Presuming that the proportionality constant does not change significantly allows us to use this relation to obtain the same iteration equation that we had before:

$$
m_{A l^{3+}, k+1}=\frac{m_{A l^{3+}, k}}{\beta_{A l^{3+}, k}+1}
$$

The only significant difference in the iteration process in this case (versus either that in which $\mathrm{Al}^{3+}$ is dominant or that in which $\mathrm{Al}(\mathrm{OH})_{4}^{-}$is dominant but switched into the basis set) is that the starting estimate is not so good. In fact, it might be very bad, off by many orders of magnitude. However, it tums out that this algorithm is very good for quickly getting to about the right order of magnitude, even if the starting estimate is off (high) by several tens of orders of magnitude. On the other hand, it is not so efficient in a close neighborhood of the solution. This makes it a good complement to Newton-Raphson iteration, which is very efficient near the solution, but which often fails to converge at all if the starting estimates are far from the solution.

Now suppose the complex $\mathrm{Al}_{13} \mathrm{O}_{4}(\mathrm{OH})_{24}^{7+}$ dominates the calculated mass balance of $\mathrm{Al}^{3+}$. Again, we do not make a basis switch. This then leads us to a result of the form:

$$
m_{A l_{13} O_{4}(O H)_{24}^{7+}, k+1}=\frac{13 m_{A l_{13} O_{4}(O H)_{24}^{7+}, k}}{\beta_{A l^{3+}, k}^{+1}}
$$

The species $\mathrm{Al}_{13} \mathrm{O}_{4}(\mathrm{OH})_{24}^{7+}$ and $\mathrm{Al}^{3+}$ are related by the reaction:

$$
\mathrm{Al}_{13} \mathrm{O}_{4}(\mathrm{OH})_{24}^{7+}+32 \mathrm{H}^{+}=13 \mathrm{Al}^{3+}+28 \mathrm{H}_{2} \mathrm{O}_{(l)}
$$

The corresponding mass action equation is:

$$
K_{A l_{33} O_{4}(O H)_{24}^{7+}}=\frac{m_{A l^{3+}}^{12} \gamma_{A l^{3+}}^{13} x_{w}^{28} \lambda_{w}^{28}}{m_{A l_{13} O_{4}(O H)_{24}^{7+} \gamma_{A l_{13} O_{4}(O H)_{24}^{7+}} m_{H^{+}}^{32} \gamma_{H^{3}}^{32}}}
$$

Using the same assumptions as before leads to the following proportionality:

$$
m_{A l^{3+}}^{13} \propto m_{A l_{13} O_{4}(O H)_{24}^{7+}}
$$

Using this as before leads to the following iteration equation:

$$
m_{A l^{3+}, k+1}=\frac{13^{\frac{1}{13}} m_{A l^{3+}, k}}{\left(\beta_{A l^{3+}, k}+1\right)^{\frac{1}{13}}}
$$


This differs from the previous results in two ways. First, an exponent now appears on the $(\beta+1)$ term. This exponent is the factor expressing the stoichiometric equivalence of the basis species corresponding to a mass balance expression to the species which dominates the calculated mass balance. Also, a factor appears in the numerator on the right hand side which is the inverse of the same exponent with a matching exponent This is the general case. These "new" elements of the equation did not explicitly appear in the previous results because the stoichiometric equivalence happened to be unity.

The exponent on the $(\beta+1)$ term is critical to the success of this method. This is because it has a large effect on the exponent of the resultant calculated concentration of the basis species. When one starts the iteration process, the value of $\beta$ may initially be something like $10^{+60}$. Use of the iteration equation ignoring this exponent would result in the calculated concentration of the basis species being lowered by 60 orders of magnitude. The effect of the exponellt on the $(\beta+1)$ is to cause it to be lowered only about 4.6 orders of magnitude. The effect of ignoring this exponent on the remainder of the calculation woukd be wild ascillation.

The effect of the factor in the numerator is less extreme. It does not affect the resulting order of magnitude. For example, $1^{\frac{1}{1}}=1,2^{\frac{1}{2}} \approx 1.41,3^{\frac{1}{3}} \approx 1.44,{ }^{\frac{1}{4}}=1.41$, and $5^{\frac{1}{5}} \approx 1.38$. In the case shown above, $13^{\frac{1}{13}} \approx 1.22$. The absence of this factor doesn't have much effect if all one is trying to do is get within about an order of magnitude of the solution before switching to another algorithm. However, it would cause convergence to fail in a close neighborhood of the solution if one attempted to use this algorithm to obtain a final solution.

Not all basis species are constrained by mass balance relations. If a species is constrained by a value for its $\log$ activity, the concentration is simply estimated from this value using the current value for the corresponding activity coefficient:

$$
\log m_{i, k+1}=\log a_{i}-\log \gamma_{i}
$$

The concentration of such a species can not change during a pass, because activity coefficients are only recalculated as the end of a pass.

A basis species whose concentration is to be adjusted to satisfy electrical balance has its concentration recalculated in a cycle after the concentrations of all other basis species have been recalculated. This will be discussed below.

The concentrations of basis species which are constrained by any other types of constraints are calculated simultaneously by solving a matrix equation. This is the case for $O_{2(g)}$ when an $E h$ or pe value is input, for cases in which heterogeneous or homogeneous equilibria must be satisfied, and cases in which a combination activity function such as $\mathrm{pHCl}$ is utilized. It is not always striclly necessary to make these calculations simultaneously, but it is more convenient to always do it this way than to deal otherwise with those cases which would allow complete or partial solution by a sequence of indjvidual calculations (whose order would have to be determined in each individual case). 
For example, suppose dissolved calcium is constrained to satisfy equilibrium with calcite and bicarbonate is constrained to satisfy a specified fugacity of carbon dioxide. We will assume that chloride is constrained by a total concentration and that the hydrogen is constrained by an input value of $p H$. The respective relevant governing equations for $\mathrm{Ca}^{2+}$ and $\mathrm{H}^{+}$are:

$$
\begin{gathered}
\log K_{\text {calcile }}=\log _{\mathrm{Ca}^{2+}}+\log \gamma_{\mathrm{Ca}^{2+}}+\log m_{\mathrm{HCO}_{3}^{-}}+\log \gamma_{\mathrm{HCO}_{3}^{-}}-\log \mathrm{H}_{\mathrm{H}^{+}}-\log \gamma_{\mathrm{H}^{+}} \\
\log K_{\mathrm{CO}_{2(\mathrm{~g})}}=\log \mathrm{HCO}_{3}^{+}+\log \gamma_{\mathrm{HCO}_{3}^{-}}+\log m_{\mathrm{H}^{+}}+\log \gamma_{\mathrm{H}^{+}}-\log \mathrm{CO}_{2}-\log x_{\mathrm{w}}-\log \lambda_{\mathrm{w}}
\end{gathered}
$$

These may be rearranged to give:

$$
\begin{gathered}
\log _{\mathrm{Ca}^{2+}}+\log m_{\mathrm{HCO}_{3}^{-}}=\log K_{\text {calcile }}-\log \gamma_{\mathrm{Ca}^{2+}}-\log \gamma_{\mathrm{HCO}_{3}^{-}}+\log m_{\mathrm{H}^{+}}+\log \gamma_{\mathrm{H}^{+}} \\
\log _{\mathrm{HCO}_{3}}-\log x_{s w}=\log K_{\mathrm{CO}_{2(g)}}-\log \gamma_{\mathrm{HCO}_{3}^{-}}-\log m_{\mathrm{H}^{+}}-\log \gamma_{\mathrm{H}^{+}}+\log {\mathrm{CO}_{2}}_{\mathrm{O}}+\log \lambda_{w}
\end{gathered}
$$

where the variables treated as the unknowns are on the left hand sides. As all these concentration values pertain to the $k+1$-th iteration, they could be so marked in the rearranged equations (but we leave them out for clarity). The mole fraction of water may also be adjusted as part of the process, using the approximate relation:

$$
\log x_{w, k+1}=\log x_{w, k}+\sum_{s^{\prime} \in S} \frac{\partial \log x_{w}}{\partial \log m_{s^{\prime}}}\left(\log m_{s^{\prime}, k+1}-\log m_{s^{\prime}, k}\right)
$$

where $S$ is the set of solute basis species whose concentrations must be solved for in this manner. This is a Taylor's series truncated to first order. For notational convenience, we make the following definition:

$$
W_{s^{\prime}}=\frac{\partial \log x_{w}}{\partial \log m_{s^{\prime}}}, \quad s^{\prime} \neq w, s_{B}
$$

Then the equation in the present example may be written as:

$$
\begin{aligned}
& \log x_{w}-W_{\mathrm{Ca}^{2+}}{ }^{\log m} \mathrm{Ca}^{2+}-W_{\mathrm{HCO}_{3}^{-}}^{\log m} \mathrm{HCO}_{3} \\
& =\log x_{w, k}-W_{\mathrm{Ca}^{2+}}{ }^{\log m_{C a^{2+}, k}}-W_{\mathrm{HCO}_{3}^{-}}{ }^{\log m_{H C O}, k}
\end{aligned}
$$

where again all the variables treated as unknowns are on the left hand side. For notational consistency, we have dropped the " $k+1$ " subscripts.

We now evaluate the necessary partial derivatives. The mole fraction of water must be expressed as a function of the concentrations of the basis species other than water. We first write this in the form: 


$$
\log x_{w}=\log \left(\frac{\Omega}{\Omega+\sum_{\substack{s^{\prime}=1 \\ s^{\prime} \neq w}}^{s_{s^{\prime}}}+\sum_{r=1}^{r_{T}} m_{s^{\prime \prime}}}\right)
$$

where $s^{\prime \prime}$ denotes the non-basis species associated with the $r$-th aqueous reaction. Partial differentiation leads to the following intermediate result:

$$
W_{s^{\prime}}=-\frac{x_{w}}{\Omega}\left(m_{s^{\prime}}-\sum_{r}^{r_{T}} \frac{b_{s^{\prime} r m_{s^{\prime \prime}}}}{b_{s^{\prime \prime} r}}-W_{s^{\prime}} \sum_{r}^{r_{T}} \frac{b_{w r} m_{s^{\prime \prime}}}{b_{s^{\prime \prime} r}}\right)
$$

where $s^{\prime}$ is a basis species other than water. Rearranging then gives the final result:

$$
W_{s^{\prime}}=\frac{-\frac{x_{w}}{\Omega}\left(m_{s^{\prime}}-\sum_{r}^{r_{T}} \frac{b_{s^{\prime} r} m_{s^{\prime \prime}}}{b_{s^{\prime \prime} r}}\right)}{\left(1-\frac{x_{w}}{\Omega} \sum_{r}^{r_{T}} \frac{b_{w r} m_{s^{\prime \prime}}}{b_{s^{\prime \prime} r}}\right)}
$$

Technically, these partial derivatives should be evaluated using data corresponding to the $k$-th iteration. However, as the method is not exact anyway and the mole fraction of water does not really vary much in the process, this is not critical.

In the above example, we have three equations in three unknowns. These equations are linear in the four log concentration variables; hence, they can be solved simultaneously by solving a corresponding matrix equation. This takes the form:

$$
\left[\begin{array}{ccc}
1 & 1 & 0 \\
0 & 1 & -1 \\
-\mathrm{W}_{\mathrm{Ca}^{2+}}-\mathrm{W}_{\mathrm{HCO}_{3}^{-}} & 1
\end{array}\right]\left[\begin{array}{c}
\log m_{\mathrm{Ca}^{2+}} \\
\log m_{\mathrm{HCO}_{3}^{-}} \\
\log x_{w}
\end{array}\right]=\left[\begin{array}{c}
R_{\mathrm{Ca}^{2+}} \\
R_{\mathrm{HCO}_{3}} \\
R_{w}
\end{array}\right]
$$

where the elements in the right hand side vector are equal to the expressions on the right hand side of eqs (266), (267), and (270). Matrix equations of this form are evaluated after new values have been calculated for the concentrations of the other basis species.

At the end of a cycle, a full set of residual functions is computed. This includes the $\beta$ array and its max norm, $\beta_{\max }$. The only non-zero elements of $\beta$ at the end of a cycle are those which pertain to mass and charge balance constraints. The elements of this vector corresponding to the former have been previously introduced. In the case of the latter, the relevant equation is: 


$$
\beta_{s_{z}}=\frac{\alpha_{s_{z}}}{\sum_{c} z_{c} m_{c}+\sum_{a}\left|z_{a}\right| m_{a}}
$$

where $s_{z}$ is the basis species so constrained, $c$ denotes cations and $a$ anions, and:

$$
\alpha_{s_{z}}=\sum_{c} z_{c} m_{c}+\sum_{a} z_{a} m_{a}
$$

The concentration of this species is then adjusted so as to make these residuals zero:

$$
m_{s_{z}, k+1}=m_{s_{z}, k}-\frac{\alpha_{s_{z}, k}}{\left|z_{s_{z}}\right|}
$$

A pass ends when one of the following occurs:

- The non-zero (mass balance) elements of $\beta$ satisfy a loose convergence test (all fall in the range $-10 \%$ to $+50 \%$ ).

- The maximum number of cycles per pass have been completed.

- A convergence function $\beta_{f u n c}$ (betfnc) indicates that iteration in the present cycle is diverging. This convergence function will be discussed later in this chapier.

At the end of a pass, the $\Sigma m$ function and the ionic strength are recalculated, the mole fraction of water is recalculated, and the activity coefficients are recaiculated. The code defines residual functions based on the magnitude of the changes in $\Sigma m$, the ionic strength, and the activity coefficients from the values pertaining to the previous pass. The sequence of passes is stops when one of the following occurs:

- The residuals defined for $\Sigma m$, the ionic strength, and the activity coefficients satisfy a loose conyergence test (all less than or equal to 0.1 ).

- The maximum number of passes have been completed.

The optimization is deemed successful if both sets of loose convergence tolerances are satisfied. The code will then execute hybrid Newton-Raphson iteration. If optimization is not successful, the code checks to see if any equilitrium constraints appear to imply unrealistically high solute concentrations. If this is the rase, the code will not attempt hybrid Newton-Raphson iteration and a message to this effect is written to the screen and output files. Otherwise, the code will execute hybrid Newton-Raphson iteration, which often succeeds even when the optimization step fails to satisfy its own convergence tolerances.

The cycle algorithm discussed in this section is an example of a first order method. This means that in a close neighborhood of the solution, one has that: 


$$
\delta_{i, k+1}=\sum_{j=1}^{n} p_{i j} \delta_{j k}
$$

where $n$ is the number of iteration variables and the $p_{i j}$ are constants.

\subsection{The Newton-Raphson Method}

The Newton-Raphson method is a well-known iterative technique for solving non-linear systems of algebraic equations (see for example Van Zeggeren and Storey, 1970; Carnahan, Luther, and Wilkes, 1969; or any introductory text on numerical methods). We will not discuss the derivation of the method here, only its application. Given a set $\boldsymbol{n}$ goveming equations and $\boldsymbol{n}$ unknowns (represented by a vector $z$ of iteration variables), one may construct a set of residual functions (represented by the vector $\alpha$ ), each member of which has a value of zero when the $n$ equations are satisfied. Both $z$ and $\alpha$ are of length $n$.

A simple example will illustrate this. Suppose we wish to solve the general quadratic equation:

$$
a x^{2}+b x+c=0
$$

This is a case in which $n=1$. Here $a, b$, and $c$ are given coefficients, and $x$ is the only unknown. The residual function can be defined as:

$$
\alpha=a x^{2}+b x+c
$$

Other definitions are possible, however. The only requirement is that they take on a value of zero when the governing equation is satisfied. We note here that the choice of definition may affect the convergence behavior.

Let $k$ be the number of iterations, such that $z_{k}$ and $\alpha_{k}$ are the iteration variable and residual function vectors on the $k$-th iteration. Let $z_{0}$ represent the set of starting estimates. An iteration step is made by calculating $z_{k+1}$ from $z_{k}$. The Newton-Raphson method does this by computing a vector of correction terms, $\delta$, by solving the matrix equation:

$$
\underline{J} \delta=-\underline{\alpha}
$$

Here $\mathcal{L}$ is the Jacobian matrix, defined by:

$$
J=\left(\frac{\partial \alpha_{i}}{\partial z_{j}}\right)
$$

where $i$ and $j$ are the matrix coordinates. In our example, this becomes:

$$
J=2 a x+b
$$

The correction term is then applied:

$$
z_{k+1}=z_{k}+\underline{\delta}_{k}
$$


If the iteration converges, all elements of both $\alpha$ and $\delta$ approach zero. It is useful to define another residual function vector $\beta$ which is identical to $\alpha$, except that some elements may be normalized to provide a better measure of convergence. It is then convenient to define $\boldsymbol{\beta}_{\max }$ and $\delta_{\max }$ as the largest absolute values of the elements of $\beta$ and $\delta$, respectively. Both $\beta_{\max }$ and $\delta_{\max }$ may then be used in tests to determine if the iteration has converged satisfactorily.

A useful measure of how well convergence is proceeding may also be constructed. The NewtonRaphson method is a second order method.This means that in a close neighborhood of the solution, one has that:

$$
\delta_{i, k+1}=\sum_{j=1}^{n} p_{i j} \delta_{j k}^{2}
$$

where the $p_{i j}$ are constants. In practice, there is usually no attempt to actually evaluate them. The significance of this is that in a close neighborhood of the solution, $\delta_{\max , k+1}$ should be much less than $\delta_{\max , k}$. The function $\delta_{f u n c}$ (the variable delfnc) is defined:

$$
\delta_{\text {func, } k+1}=1-\left(\frac{\delta_{\text {max, } k+1}}{\delta_{\text {max, }, k}}\right)
$$

may therefore be expected to approach (from below) a value of unity if the iteration is converging very rapidly (as theory suggests when the cross terms describing the evolution of the correction vector are small; i. e., the $p_{i j}$ are small for the case $i \neq j$ ). Convergence to a lesser value, say $\approx$ 0.72 instead of $=0.99$ is not unknown. This may imply non-negligible cross terms or an error in writing the Jacobian matrix. It also may result from modifications to the basic Newton-Raphson method, such as we have introduced by updating the activity coefficients between Newton-Raphson steps. The function, $\beta_{f u n c}$ (the variable betinc) is defined similarly:

$$
\beta_{f u n c, k+1}=1-\left(\frac{\beta_{\max , k+1}}{\beta_{\max , k}}\right)
$$

and has essentially the same properties.

The use of a pure Newton-Raphson method would require the activity coefficients and their associated model equations to be brought directly into the set of $n$ equations and $n$ unknowns solved by the method, either directly or by substitution. In a previous section in this chapter, we noted that there was a problem in expanding the current set of master iteration variables in that the activity coefficients would have to be calculated uefore the concentrations of the non-basis species, and vice versa. This problem precludes taking care of the activity coefficients by a substinution mechanism that leaves the current set of master iteration variables unchanged. One would have to instead treat the activity coefficients themselves as master iteration variables. These are many, so this would not be very convenient. Alternatively, one could treat the ionic strength ar a master iteration variable, but this would only suffice for simple extended Debye-Hückel formalisms that are only valid in dilute solutions. We have instead chosen to hybridize the Newton-Raphson 
method by simply updating the activity coefficients be:ween Newton-Raphson steps. In practice, this seems to work quite well, except in some extremely concentrated solutions.

The EQLIB module newton.f oversees the Newton-Raphson iteration for EQ3NR. Fellow EQLIB module nrstep.f is called to make a single Newton-Raphson step, and fellow EQLIB module ngcadv. $f$ recomputes activity coefficients and computes the number of moles of dependent species. The latter module is called between Newton-Raphson steps, in accordance with the single update method that was described earlier in this chapter. The EQ3NR module betas.f computes the residual functions, and the EQ3NR module matrix.f writes the Jacobian matrix.

The maximum number of iterations in a Newton-Raphson calculation is determined by the input file variable itermx. This has a default value $r .30$ in EQ3NR. Convergence is achieved when $\beta_{\text {max }}$ is less than the tolerance parameter tolbt, $\delta_{\text {max }}$ is less than the tolerance parameter told!, and max norms on the changes in the $\Sigma m$ function, the ionic strength, and the activity coefficients are all fess than tofbt. The tolerance parameters tolbt and told both appear on the input file, and both have a default value of $1 \times 10^{-6}$.

\subsection{Derivation of Residual Functions and the Jacobian Matrix}

In this section, we shall derive the residual functions and the Jacobian matrix for the NewtonRaphson iteration procedures used by the EQ3NR code. Given a set of governing equations and an equal number of unknowns, there is no unique way to formulate residuals and Jacobians. The number of equations and unknowns may be reduced by means of substitutions. Furthermore, one may then construct the residual functions in any number of ways. Once the residual functions have been chosen, the form of the Jacobian is determined according to the partial derivatives of these functions.

We will now take each remaining governing equation, construct a corresponding pair of residual functions ( $\alpha$ and $\beta$ ), and derive the corresponding row of elements in the Jacobian matrix by partial differentiation. The $\alpha$ residuals are the true Newton-Raphson residual functions and are the subject of partial differentiation to define the Jacobian matrix. The $\beta$ residuals are better measures of satisfactory convergence.

\subsubsection{Mass Balance}

This may be applied to any aqueous species in the basis set $\left(s=1\right.$ through $\left.s_{Q}\right)$ except water (w), $H^{+}$, and $O_{2(g)}$ (the $s_{B}$-th species). Mass balance is specified as the governing equation by setting the corresponding jflag value to 0 and entering a total concentration on the molal scale $\left(m_{T, s}\right)$. Alternatively, one may enter total concentration in other units using other jflag values (molar, jflag $=1 ; \mathrm{mg} / \mathrm{L}$, jflag $=2 ; \mathrm{mg} / \mathrm{kg}$ of solution, $\mathrm{jflag}=3$ ), which EQ3NR will then recompute into molality and set jflng to zero. The governing equation can be written as:

$$
m_{T, s}=\sum_{s^{\prime}=1}^{s_{Q}} u_{s^{\prime} s} m_{s}+\sum_{s^{\prime \prime}=s_{Q}+1}^{s_{T}} u_{s^{\prime \prime} s^{m}} m_{s^{\prime \prime}}
$$

The residual functions are defined by: 


$$
\begin{gathered}
\alpha_{s}=-m_{T, s}+\sum_{s^{\prime}=1}^{s_{Q}} u_{s^{\prime} s^{m_{s}}}+\sum_{s^{\prime \prime}=s_{Q}+1}^{s_{T}} u_{s^{\prime \prime} s^{m_{s}}} \\
\beta_{s}=\frac{\alpha_{s}}{m_{T, s}}
\end{gathered}
$$

where in the last part of eqs (288) and (289) it is implied that $s^{\prime \prime}=s_{r}$. From this point, we may use the relations developed above to derive the following Jacobian elements:

$$
\begin{aligned}
& J_{s w}=\frac{\partial \alpha_{s}}{\partial \log x_{w}} \\
& =\sum_{r=1}^{r} u_{s^{\prime \prime}} \frac{\partial m_{s^{\prime \prime}}}{\partial \log x_{w}} \\
& =-2.303 \sum_{r=1}^{r_{T}} \frac{u_{s^{\prime \prime} s^{m^{\prime \prime}} b_{w r}}}{b_{s^{\prime \prime} r}} \\
& =-2.303 \sum_{r=1}^{r_{T}} b_{w r} H_{s r} \\
& J_{s s_{B}}=\frac{\partial \alpha_{s}}{\partial \log f_{O_{2}}} \\
& =\sum_{r=1}^{r_{T}} u_{s^{n}} \frac{\partial m_{s^{\prime \prime}}}{\partial \log f_{O_{2}}} \\
& =2.303 \sum_{r=1}^{r} \frac{u_{s^{\prime \prime} s} m_{s^{\prime \prime}} b_{s_{B}} r}{b_{s^{\prime \prime} r}} \\
& =2.303 \sum_{r=1}^{r_{T}} b_{s_{B} r} H_{s r}
\end{aligned}
$$

and for $s \neq w, s_{B}$ : 


$$
\begin{aligned}
& J_{s s^{\prime}}=\frac{\partial \alpha_{s}}{\partial \log m_{s^{\prime}}} \\
& =\sum_{s^{n \prime}=1}^{s_{Q}} u_{s^{\prime \prime \prime}} \cdot \frac{\partial m_{s^{\prime \prime \prime}}}{\partial \log m_{s^{\prime}}}+\sum_{s^{\prime \prime}=s_{Q}+1}^{s_{T}} u_{s^{\prime \prime}} \frac{\partial m_{s^{\prime \prime}}}{\partial \log m_{s^{\prime}}} \\
& =-2.303\left(u_{s^{\prime} s^{m}} m_{s^{\prime}}-\sum_{r=1}^{r_{T}} \frac{u_{s^{\prime \prime}} m_{s^{\prime \prime}} b_{s^{\prime} r}}{b_{s^{\prime \prime} r}}\right) \\
& =-2.303\left(u_{s^{\prime} s^{\prime}} m_{s^{\prime}}-\sum_{r=1}^{r_{T}} b_{s^{\prime} r} H_{s r}\right)
\end{aligned}
$$

Here $u_{s^{n 1}} s^{\prime}=1.0$ if $s^{\prime}=s^{\prime \prime \prime}$, otherwise $u_{s^{* 11}} s^{\prime}=0$.

\subsubsection{Electrical Balance}

This governing equation may be applied to one of the ions in the aqueous species basis set, here denoted by $s$. Apart from the definition of the $\beta$ residual, the treatment is exactly analogous to that for mass balance. The governing equation can be written as:

$$
\sum_{s^{\prime}=1}^{s_{Q}} z_{s^{\prime}} m_{s}+\sum_{s^{n}=s_{Q}+1}^{s_{T}} z_{s^{\prime \prime}} m_{s^{\prime \prime}}=0
$$

The residual functions are defined by:

$$
\begin{aligned}
& \alpha_{s}=\sum_{s^{\prime}=1}^{s_{Q}} z_{s^{\prime}} m_{s}+\sum_{s^{\prime \prime}=s_{Q^{+1}}}^{s_{T}} z_{s^{\prime \prime}} m_{s^{\prime \prime}} \\
& \beta_{s}=\frac{\alpha_{s}}{\sum_{s^{\prime}=1}^{s_{Q}}\left|z_{s^{\prime}}\right| m_{s^{\prime}}+\sum_{s^{\prime \prime}=s_{Q}+1}^{s_{T}}\left|z_{s^{\prime \prime}}\right| m_{s^{\prime \prime}}}
\end{aligned}
$$

The corresponding Jacobian elements are as follows: 


$$
\begin{aligned}
J_{s w} & =\frac{\partial \alpha_{s}}{\partial \log x_{w}} \\
& =-2.303 \sum_{r=1}^{r_{T}} b_{w r} H_{z r} \\
J_{s s_{B}} & =\frac{\partial \alpha_{s}}{\partial \log f_{O_{2}}} \\
& =2.303 \sum_{r=1}^{r_{T}} b_{s_{U} r} H_{z r}
\end{aligned}
$$

and for $s \neq w, \mathrm{~S}_{B}$ :

$$
\begin{aligned}
J_{s s^{\prime}} & =\frac{\partial \alpha_{s}}{\partial \log m_{s^{\prime}}} \\
& =-2.303\left(z_{s^{\prime}} m_{s^{\prime}}-\sum_{r=1}^{r_{T}} b_{s^{\prime},} H_{z r}\right)
\end{aligned}
$$

\subsubsection{The Mole Fraction Of Water}

The governing equation can be written as:

$$
x_{w}=\frac{\Omega}{\Omega+\sum_{\substack{s=1 \\ s \neq w}}^{s_{T}} m_{s}}
$$

where $\Omega$ is the number of moles of solvent water comprising a mass of $1 \mathrm{~kg}(\Omega \approx 55.51)$ and $s_{T}$ is the number of aqueous species in the solution. The corresponding residual functions are defined as: 


$$
\alpha_{w}=\log \left(\frac{\Omega}{\Omega+\sum_{\substack{s=1 \\ s \neq w}}^{s_{T}} m_{s}}\right)-\log x_{w}
$$

Because it is necessary to distinguish between basis species and non-basis species, it is helf $i l$ to write the equation for the first residual function in the slightly expanded form:

$$
\alpha_{w}=\log \left(\frac{\Omega}{\Omega+\sum_{\substack{s^{\prime}=1 \\ s^{\prime} \neq w}}^{s_{Q}} m_{s^{\prime}}+\sum_{r=1}^{r_{T}} m_{s^{\prime \prime}}}\right)-\log x_{w}
$$

In the following equations, we will take $s^{\prime}$ and $s^{\prime \prime \prime}$ to be basis species other than water. The following Jacobian elements are then obtained:

$$
\begin{aligned}
& J_{w w}=\left(\frac{1}{\Omega}\right)\left(\frac{\Omega}{\Omega+\sum_{s^{\prime}=1}^{s_{Q}} m_{s^{\prime}}+\sum_{r=1}^{s^{\prime} \neq w} m_{s^{\prime \prime}}}\right)\left(\sum_{r=1}^{\left.\sum_{T} \frac{b_{w r} m_{s^{\prime \prime}}}{b_{s^{\prime \prime} r}}\right)-1.0}\right. \\
& J_{w s^{\prime \prime}}=\left(-\frac{1}{\Omega}\right)\left(\frac{\Omega^{s_{Q}}}{\Omega+\sum_{s^{\prime}=1}^{m_{s^{\prime}}+\sum_{r=1}^{r_{T}} m_{s^{\prime \prime}}}}\right)\left(m_{s^{\prime \prime \prime}}-\sum_{r=1}^{r_{T} \frac{b_{s^{\prime \prime \prime}} m_{s^{\prime \prime}}}{b_{s^{\prime \prime} r}}}\right)
\end{aligned}
$$

Note that these Jacobian elements differ from the corresponding set of partial detivatives used in the truncated Taylor's expansion as part of the pre-Newton-Raphson optimization. In the present case, $\log x_{w}$ is treated as an independent variable. In the previous case, it was not. 


\subsubsection{Specified Free Concentration}

This represents the jflag $=4$ option for $s=1$ through $s_{Q}$, except when $s$ is water $(w)$ or $O_{2(g)}\left(s_{B}\right)$. The free concentration is in molality. If it is initially entered in molarity (jflag $=S$ ), EQ3NR will converts it to molality and resets jflag to 4 before beginning Newton- Raphson iteration. The governing equation is just the identity:

$$
m_{s}=m_{s}
$$

Hence, the corresponding residual functions are given by:

$$
\begin{aligned}
& \alpha_{s}=0 \\
& \beta_{s}=0
\end{aligned}
$$

In order to prevent singularity in the Jacobian, we set:

$$
J_{s s}=1.0
$$

\subsubsection{Specified Thermodynamic Activity}

This represents the jflag $=16$ option for $s=1$ through $s_{Q}$, except when $s$ is water $(w)$ or $O_{2(g)}\left(s_{B}\right)$. This option is most frequently employed with $H^{+}$in order to specify a $p H$ value $\left(p H=-\log a_{H^{+}}\right.$). The governing equation is:

$$
\log m_{s}+\log \gamma_{s}=\log a_{s}
$$

The residual functions are:

$$
\begin{gathered}
\alpha_{s}=-\log a_{s}+\log m_{s}+\log \gamma_{s} \\
\beta_{s}=\alpha_{s}
\end{gathered}
$$

The only non-zero Jacobian element is:

$$
J_{s s}=1.0
$$

\subsubsection{Log Activity Combination}

Recall that the activity combination parameter is defined by:

$$
\kappa_{i j}=\left|z_{j}\right| \log a_{i}-\frac{z_{i}{ }^{2} j}{\left|z_{j}\right|} \log a_{j}
$$

We will identify $i$ as the basis species $s$ to which this constraint is applied, and $s^{*}$ as the other basis species invoiveci. The governing equation can then be written as: 


$$
\log m_{s}=\frac{x_{s s^{*}}}{\left|z_{s *}\right|}-\log \gamma_{s}+\frac{z_{s}}{z_{s *}} \log m_{s^{*}}+\frac{z_{s}}{z_{s^{*}}} \log \gamma_{s^{*}}
$$

Hence, the corresponding residual functions are given by:

$$
\begin{gathered}
\alpha_{s}=\frac{x_{s s^{*}}}{\left|z_{s^{*}}\right|}-\log \gamma_{s}+\frac{z_{s}}{z_{s^{*}}} \log m_{s^{*}}+\frac{z_{s}}{z_{s^{*}}} \log \gamma_{s^{*}}-\log m_{s} \\
\beta_{s}=\alpha_{s}
\end{gathered}
$$

The corresponding non-zero elements of the Jacobian matrix are then:

$$
\begin{aligned}
& J_{s s}=-1.0 \\
& J_{s s^{*}}=\frac{z_{s}}{z_{s^{*}}}
\end{aligned}
$$

\subsubsection{Mean Log Activity}

The mean log activity of two oppositely charge ions $i$ and $j$ can be written as:

$$
\log a_{ \pm, i j}=\frac{\left|z_{j}\right| \log a_{i}+\left|z_{i}\right| \log a_{j}}{\left|z_{i}\right|+\left|z_{j}\right|}
$$

We will identify $i$ as the basis species $s$ to which this constraint is applied, and $s^{*}$ as the other basis species involved. The governing equation can then be written as:

$$
\log m_{s}=\frac{\left|z_{s}\right|+\left|z_{s^{*}}\right|}{\left|z_{s^{*}}\right|} \log a_{ \pm, s s^{*}}-\log \gamma_{s}-\left|\frac{z_{s}}{z_{s^{*}}}\right| \log m_{s^{*}}-\left|\frac{z_{s}}{z_{s^{*}}}\right| \log \gamma_{s^{*}}
$$

Hence, the corresponding residual functions are given by:

$$
\begin{gathered}
\alpha_{s}=\frac{\left|z_{s}\right|+\left|z_{s *}\right|}{\left|z_{s *}\right|} \log a_{ \pm, s s^{*}}-\log \gamma_{s}-\left|\frac{z_{s}}{z_{s^{*}}}\right| \log m_{s^{*}}-\left|\frac{z_{s}}{z_{s^{*}}}\right| \log \gamma_{s^{*}}-\log m_{s} \\
\beta_{s}=\alpha_{s}
\end{gathered}
$$

The corresponding non-zero elements of the Jacobian matrix are then:

$$
\begin{gathered}
J_{s s}=-1.0 \\
J_{s s^{*}}=-\left|\frac{z_{s}}{z_{s^{*}}}\right|
\end{gathered}
$$




\subsubsection{Equilibrium With A Pure Mineral}

This option (jflag $=19)$ may be specified for any aqueous species denoted by $s=1$ through $s_{Q}$, except when $s$ is water $(w)$. Let $\phi$ denote the mineral in question. The goveming equation is:

$$
\log K_{\phi}=b_{w \phi}\left(\log x_{w}+\log \lambda_{w}\right)+b_{s_{B} \phi} \log f_{O_{2}}+\sum_{\substack{s^{\prime}=1 \\ s^{\prime} \neq w, s_{B}}}^{s_{Q}} b_{s^{\prime} \phi}\left(\log m_{s^{\prime}}+\log \gamma_{s^{\prime}}\right)
$$

The residual functions are defined as:

$$
\begin{gathered}
\alpha_{s}=\frac{1}{b_{s \phi}}\left(\log K_{\phi}-b_{w \phi}\left(\log x_{w^{\prime}}+\log \lambda_{w^{\prime}}\right)-b_{s_{B^{\phi}}} \log f_{O_{2}}\right. \\
\left.-\sum_{\substack{s^{\prime}=1 \\
s^{\prime} \neq w, s_{B}}}^{s_{Q}} b_{s^{\prime} \phi}\left(\log m_{s^{\prime}}+\log \gamma_{s^{\prime}}\right)\right) \\
\beta_{s}=\alpha_{s}
\end{gathered}
$$

The corresponding Jacobian elements are then:

$$
J_{s s^{\prime}}=-\frac{b_{s^{\prime} \phi}}{b_{s \phi}}
$$

The residual function $\alpha_{s}$ defined in eq (327) has in a sense been normalized by dividing by the stoichiometric reaction coefficient $b_{s \phi}$. This makes the residual equivalent to the difference between the calculated and current values of $\log m_{s}$, independent of how the reaction has been written. This avoids some potential numerical scaling problems. Other options involving mass action equations are treated in the same manner.

\subsubsection{Equilibrium With A Solid Solution End-member Component}

This option (jflag $=20$ ) may be specified for any aqueous species denoted by $s=1$ through $s_{Q}$, except when $s$ is water ( $w$ ). The treatment is closely analogous to that for equilibrium with a pure mineral. Let $\sigma$ and $\psi$ denote the end-member and solid solution phase, respectively. The governing equation contains an additional term in the mole fraction and activity coefficient of the solid solution end-member and is given by: 


$$
\begin{aligned}
& \log K_{\sigma \psi}=b_{\sigma \psi \sigma \psi}\left(\log x_{\sigma \psi}+\log \lambda_{\sigma \psi}\right)+b_{w \sigma \psi}\left(\log x_{w}+\log \lambda_{w}\right)+b_{s_{B} \sigma \psi} \log f_{O_{2}} \\
& +\sum_{\substack{s^{\prime}=1 \\
s^{\prime} \neq w, s_{P}}}^{s_{Q}} b_{s^{\prime} \sigma \Psi}\left(\log m_{s^{*}}+\log \gamma_{s^{*}}\right)
\end{aligned}
$$

The residual functions are defined as:

$$
\begin{gathered}
\alpha_{s}=\frac{1}{b_{s \sigma \psi}}\left(\log K_{\sigma \psi}-b_{\sigma \psi \sigma \psi}\left(\log x_{\sigma \psi}+\log \lambda_{\sigma \psi}\right)-b_{\psi \sigma \psi}\left(\log x_{w}+\log \lambda_{w}\right)-\right. \\
-b_{s_{B} \sigma \psi} \log f_{O_{2}}-\sum_{s^{\prime}=1}^{s_{Q}} b_{s^{\prime} \sigma \psi}\left(\log m_{s^{\prime}}+\log \gamma_{s^{\prime}}\right) \\
s^{\prime} \neq w, s_{B} \\
\beta_{s}=\alpha_{s}
\end{gathered}
$$

The corresponding Jacobian elements are then:

$$
J_{s s^{\prime}}=-\frac{b_{s^{\prime} \sigma \Psi}}{b_{s \sigma \psi}}
$$

\subsubsection{Equilibrium With A Gas}

This option (jflag $=21$ ) may be specified for any aqueous species denoted by $s=1$ through $s_{Q}$, except when $s$ is water $(w)$. The treatment is closely analogous to that for equilibrium with a pure mineral. Let $g$ denote the gas in question. The governing equation contains an additional term in the fugacity of this gas and is given by:

$$
\log K_{g}=b_{g g} \log f_{g}+b_{w g}\left(\log x_{w}+\log \lambda_{w}\right)+b_{s_{B} g} \log f_{O_{2}}+\sum_{\substack{s^{+}=1 \\ s^{\prime} \neq w, s_{B}}}^{s_{Q}} b_{s^{\prime} g}\left(\log m_{s^{\prime}}+\log \gamma_{s^{\prime}}\right)
$$

The residual functions are defined as: 


$$
\begin{gathered}
\alpha_{s}=\frac{1}{b_{s g}}\left(\log K_{g}-b_{g g} \log f_{g}-b_{w g}\left(\log x_{w}+\log \lambda_{w^{\prime}}\right)\right. \\
-b_{s_{B} g} \log f_{O_{2}}-\sum_{\substack{s^{\prime}=1 \\
s^{\prime} \neq w, s_{B}}}^{s_{Q}} b_{s^{\prime} g}\left(\log m_{s^{\prime}}+\log \gamma_{s^{\prime}}\right) \\
\beta_{s}=\alpha_{s}
\end{gathered}
$$

The corresponding Jacobian elements are then:

$$
J_{s s^{\prime}}=-\frac{b_{s^{\prime} g}}{b_{s g}}
$$

\subsubsection{Concentration Fixed By Internal Equilibrium}

This option (jflag $=27$ ) excludes the species to which it is applied, which must be in the auxiliary basis set, and its ion-pairs and complexes from the mass balance of the corresponding basis species to which it is linked by its own associated reaction (usually a strict basis species). This is a good choice for dissolved gas species such as $O_{2(a q)}$ and $H_{2(a q)}$ If $H S$ is an auxiliary basis species with jflag $=27$ and it is linked to $\mathrm{SO}_{4}{ }^{2-}$, then $\mathrm{HS}$ and its "complexes" (other species whose reactions link them to this species) are not included in calculating the $\mathrm{SO}_{4}{ }^{2-}$ mass balance. If instead one chooses $\mathbf{j f l a g}=30$ for $H S$, they are included.

Let $s$ be the auxiliary basis species constrained by jffag $=27$, and let $r$ denote its associated reaction. The governing is then:

$$
\begin{gathered}
\log K_{r}=b_{s r}\left(\log m_{s^{\prime}}+\log \gamma_{s}\right)+b_{w r}\left(\log x_{w}+\log \lambda_{w}\right) \\
+b_{s_{B} r} \log f_{O_{2}}+\sum_{\substack{s^{\prime}=1 \\
s^{\prime} \neq w, s_{B^{\prime}} s}}^{s_{Q}} b_{s^{\prime} r}\left(\log m_{s^{\prime}}+\log \gamma_{s^{\prime}}\right)
\end{gathered}
$$

The residual functions are defined as: 


$$
\begin{gathered}
\alpha_{s}=\frac{1}{b_{s r}}\left(\log K_{r}-b_{s r}\left(\log m_{s}+\log \gamma_{s}\right)-b_{w r}\left(\log x_{w}+\log \lambda_{w}\right)\right. \\
\left.-b_{s_{B} r} \log f_{O_{2}}-\sum_{\substack{s^{\prime}=1 \\
s^{\prime} \neq w, s_{B}, s}}^{s_{Q}} b_{s^{\prime} r}\left(\log m_{s^{\prime}}+\log \gamma_{s^{\prime}}\right)\right) \\
\beta_{s}=\alpha_{s}
\end{gathered}
$$

The corresponding Jacobian elements are then:

$$
J_{s s^{\prime}}=-\frac{b_{s^{\prime} r}}{b_{s r}}
$$

\subsubsection{Specified Log Oxygen Fugacity}

This option (iopt1 $=0$ ) allows direct specification of the log oxygen fugacity. The governing equation is just the identity:

$$
\log f_{O_{2}}=\log f_{O_{2}}
$$

The residuals are:

$$
\begin{aligned}
& \alpha_{s}=0 \\
& \beta_{s}=0
\end{aligned}
$$

The only non-zero Jacobian element in the corresponding row is:

$$
J_{s s}=1.0
$$

\subsubsection{Specified $E \boldsymbol{h}$}

This option (iopt1 = -1) allows indirect specification of the log oxygen fugatity. If pe is specifed (iopt1 $=-2$ ), EQ3NR converts it to $E h$ before it does the Newton-Raphson iteration. Letting $F$ be the Faraday constant, $R$ the gas constant, and $T$ the absolute temperature, the governing equation can be written as:

$$
\log f_{O_{2}}=\frac{4 F E h}{2.303 R T}+\log K_{E h}+2\left(\log x_{w}+\log \lambda_{w}\right)-4\left(\log m_{H^{+}}+\log \gamma_{H^{+}}\right)
$$

The residual functions are: 


$$
\begin{gathered}
\alpha_{s_{B}}=\frac{4 F E h}{2.303 R T}+\log K_{E h}+2\left(\log x_{w}+\log \lambda_{w}\right)-4\left(\log m_{H^{+}}+\log \gamma_{H^{+}}\right)-\log f_{O_{2}} \\
\beta_{s}=\alpha_{s}
\end{gathered}
$$

The non-zero Jacobian elements in the corresponding row are:

$$
\begin{gathered}
J_{s_{B} w}=2 \\
J_{s_{B} H^{+}}=-4 \\
J_{s_{B} s_{B}}=-1
\end{gathered}
$$

\subsubsection{Oxygen Fugacity Fixed by An Aqueous Redox Couple}

.This represents the iopt1 $=1$ option. Here $s$ is restricted to $s_{B}$. The couple is specified on the input file by setting uredox to the name of the auxiliary basis species which comprises half of the couple (the other half is automatically the corresponding strict basis species). Let $r$ denote the reaction associated with the auxiliary basis species $\left(s_{r}\right)$ in the desired couple. The governing is then:

$$
\begin{gathered}
\log K_{r}=b_{s_{r} r}\left(\log m_{s_{r}}+\log \gamma_{s_{r}}\right)+b_{w r}\left(\log x_{w}+\log \lambda_{w}\right) \\
+b_{s_{B} r} \log f_{O_{2}}+\sum_{\substack{s^{\prime}=1 \\
s^{\prime} \neq w, s_{B}, s_{r}}}^{s_{Q}} b_{s^{\prime} r}\left(\log m_{s^{\prime}}+\log \gamma_{s^{\prime}}\right)
\end{gathered}
$$

The residual functions are defined as:

$$
\begin{gathered}
\alpha_{s}=\frac{1}{b_{s_{B} r}}\left(\log K_{r} \cdot b_{s_{r} r}\left(\log m_{s_{r}}+\log \gamma_{s_{r}}\right)-b_{w r}\left(\log x_{w}+\log \lambda_{w}\right)\right. \\
\left.-b_{s_{B} r} \log f_{O_{2}}-\sum_{\substack{s^{\prime}=1 \\
s^{\prime} \neq w^{\prime}, s_{n^{\prime}, s_{r}}}}^{s_{Q}} b_{s^{\prime} r}\left(\log m_{s^{\prime}}+\log \gamma_{s^{\prime}}\right)\right) \\
\beta_{s}=\alpha_{s}
\end{gathered}
$$


The corresponding Jacobian elements are then:

$$
J_{s s^{\prime}}=-\frac{b_{s^{\prime} r}}{b_{s r}}
$$

\subsection{Methods for Crash Diagnostics}

The iteration procedure is good enough that divergence almost always results from the input of a bad set of input constraints. EQ3NR screens the input prior to Newton-Raphson iteration, but some cases are not sufficiently obvious to be caught at this stage. When the iteration diverges, EQ3NR calls the module ndiagx.f, which examines fnal state of the iteration process in an attempt to generate diagnostics to write on the screen and output îles. Barring the generation of a useful diagnostic, the user should examine the iteration suinmary on the output file for clues to the cause of the situation.

An iteration crash is generally the result of an iteration variable "blowing up" or, more commonly, "blowing down." "Blowing up" means that the value of an iteration variable or its corresponding residual function, usually a mass balance residual, is increasing to the point where the matrix equation can no longer be solved by the computer and iteration must terminate. Sometimes in this situation the operating system may terminate the code execution due to an overflow condition. If a variable "blows down," it probably means that no physical solution exists for the problem as posed. Because the primary iteration variables are logarithmic, underflow can only occur when these variables are exponentiated. When this happens, the code is usually stopped by encountering what appears to be a singular Jacobian matrix.

"Blow down" occurs for example when the problem calls for the concentration of a species to be adjusted to satisfy electrical balance, but this balance can only be achieved if the resulting concentration takes a negative value. An iteration process which only allows adjustments to log concentrations will never allow the generation of concentration value which is non-positive. What happens instead is that the calculated adjustments to the log concentration become large and negative. These are truncated to a value of $-\delta^{\prime}$ (-screwd). As this adjustment truncation parameter has a magnitude of 2.0 , the largest adjustment (element of the del array) takes on a value of -2.0 . This can be seen in the iteration summary that is printed on the output file as the code executes Newton-Raphson iteration.

An iteration block prinied during this iteration is exemplified by the following:

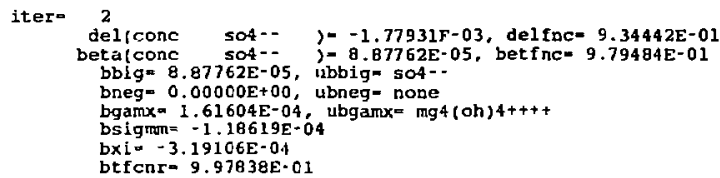

This is taken from the sea water test case that was presented as the first example in Chapter 7. The del element with the largest magnitude and the beta element with the largest magnitude are printed first. We see that in both cases, these are the elements corresponding to the concentration of the basis species $\mathrm{SO}_{4}^{2-}$. "Blow down" would be evident if the del output in this block were to 
take on a value of -2.0 (usually for the last several iterations before the process is terminated). "Blow up" would be evident if the beta output in this block were to take on very high positive values. The variables delfnc and betfnc are convergence functions for the del and beta arrays, respectively. They usually start at small positive values less than unity and then approach unity if the iteration process converges. If one or the other takes on sustained negative values, the iteration process usually diverges. Here bbig is the largest positive mass balance tesidual (uimbig identifying the corresponding species) and bneg is the negative mass balance residual (if any) with the largest magnitude (ubneg identifying the corresponding species). The variable bgamx is an aqueous species activity coefficient residual function (the max norm on the absolute values of the differences between current and previous values of the activity coefficients of the aqueous species); ubgamx identifies the corresponding species. Also, bsigm is a residual on the $\Sigma m$ function (difference between the current and previous values), and bxi is a similar residual on the ionic strength. The variable btfncr is similar to betfnc, but measures the convergence in a pure Newton-Raphson step. Usually betfnc has a smaller value than btfncr because any adjustment of activity coefficient values in between Newton-Raphson steps tends to reduce the overall reduction in the residual functions. 


\section{Code Architecture and Flow of Execution}

The purpose of the present chapter is to describe the structure of the software itself. This material is primarily included because it is required as part of the documentation to satisfy NUREG-0856 (Silling, 1983). It does not provide anything necessary for the typical code user. It may be helpful to those few users who desire to modify the code for whatever purpose.

In the present description, we will not make it a point to describe the role and function of every module in the source code. For such descriptions, the reader is referred to the relevant glossaries of modules. For EQ3NR modules, see Appendix B of the present report. For EQLIB modules, see Appendix A of the EQ3/6 Package Overview and Installation Guide (Wolery, 1992). The purpose here is to describe the main features and essential aspects of the structure of the code. The purpose is not to provide detailed design documentation. Readers who want more detailed information are invited to examine the source code itself, which is reasonably well-documented internally.

The main program is the module eq3nr.f. It directs the overall process of code execution. This is illustrated in Figure 4, which is a simplified flow diagram of the code from the point of view of this module. The first function of eq $3 \mathrm{nr}$. $\mathrm{f}$ is to get the time and date, which it does by calling the EQLIB module timdat.f. This module contains UNIX-dependent code. The main program then writes the code name and version identification, the copyright notice, and the time and date information to the screen and output files. Its next step is to initialize the dimensioning variables which correspond to the dimensioning parameters. Dimensioning variables are used to pass dimensioning data in the calling sequences of called modules; FORTRAN does not generally allow parameters (in the special FORTRAN sense) to be passed in calling sequences.

Recall that EQ3NR will run multiple problems stacked on a single input file. The line " 20 continue" is a retum point in the main program to which execution flows after a single problem has been solved. After this point, the code initializes the relevant arrays and variables, setting their contents to zero or some other appropriate null value equivalent. The purpose of this is to provide a clean slate for the solution of the next problem. The main program then calls module rdinp.f to read the first or a following problem on the input file. If no new problem is found, the problem execution stage is presumed to be finished. The main program then gets the current time and date and writes the starting and ending times and dates to the screen and output files. After that, it makes a "normal exit" and execution stops.

Module rdinp.I does the following. If the input file has not beer opened on a previous call, it opens it. Using an EQLIB module called stripl.f, it copies the input file to a file called inputs, deleting any comment line:: The original input file is then closed. The code then subsequently reads the stripped input file instead. Module rdinp.f looks at the first line of this file to see if it is in "W" or " $D$ " form. t. It then repositions the file pointer at the top of the file. 


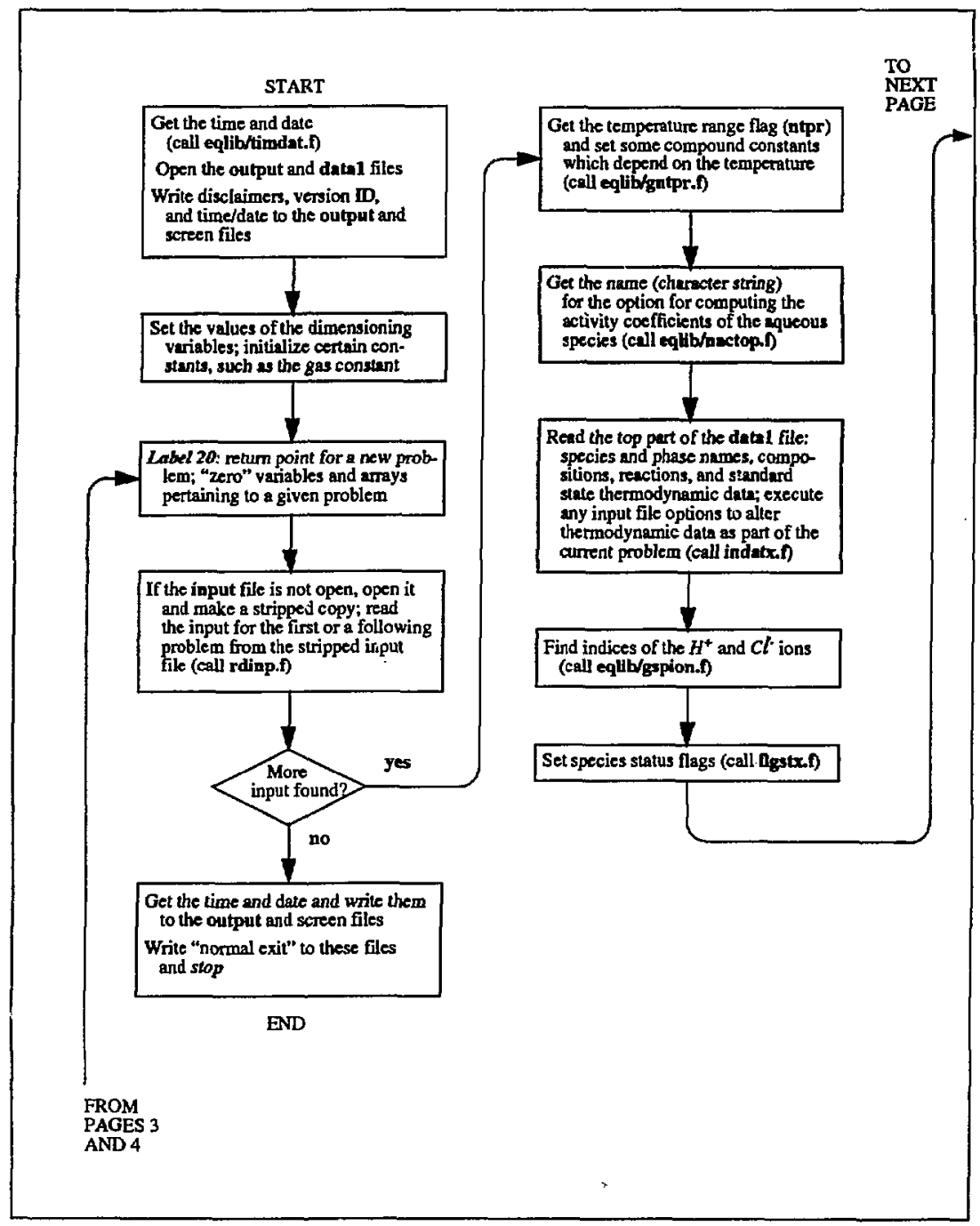

Figure 4 (page 1 of 4). Simplified flow diagram of the EQ3NR main program (eq3nr.f). 


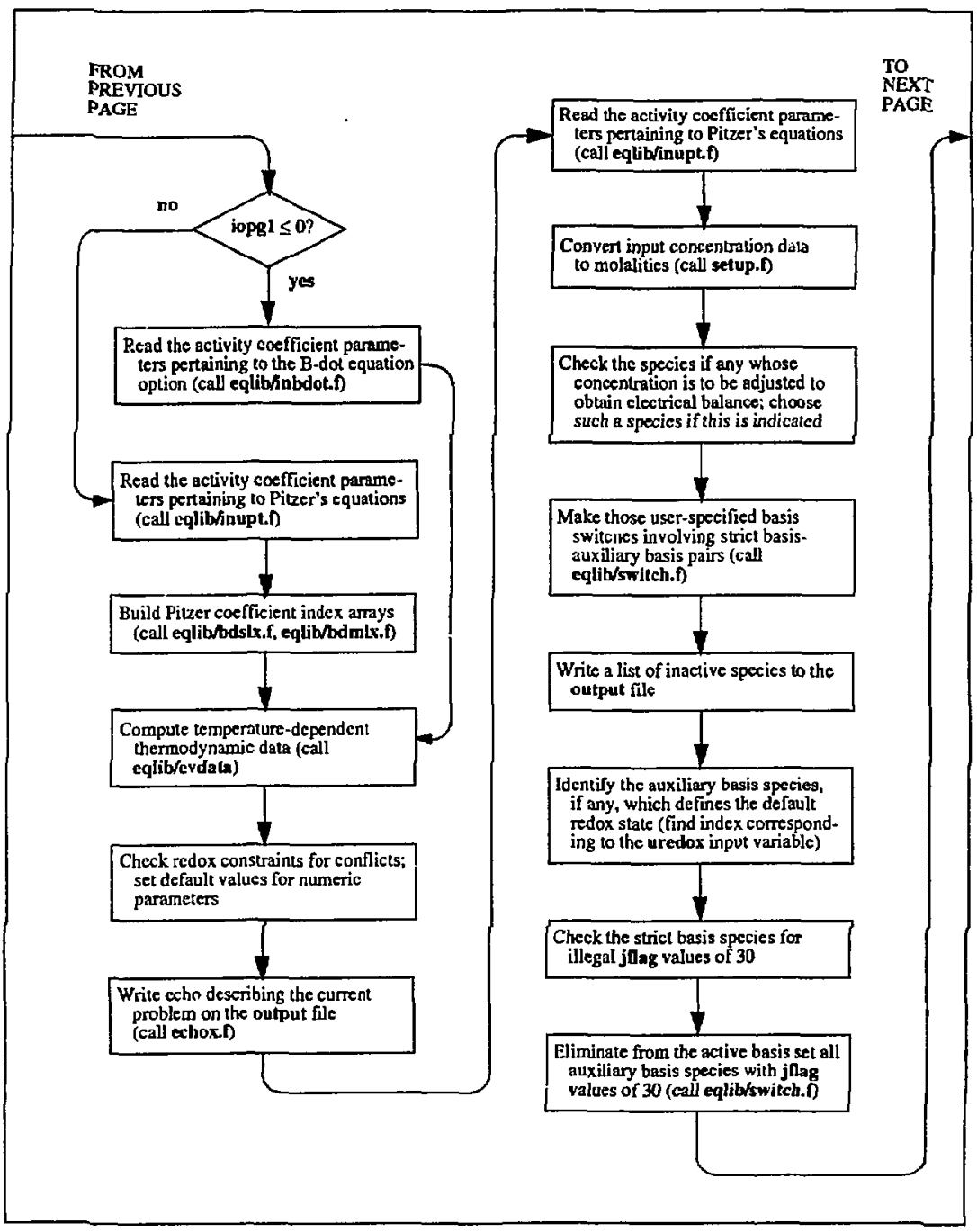

Figure 4 (continted, page 2 of 4). Simplified tlow diagram of the EQ3NR main program (eq3nr.f). 


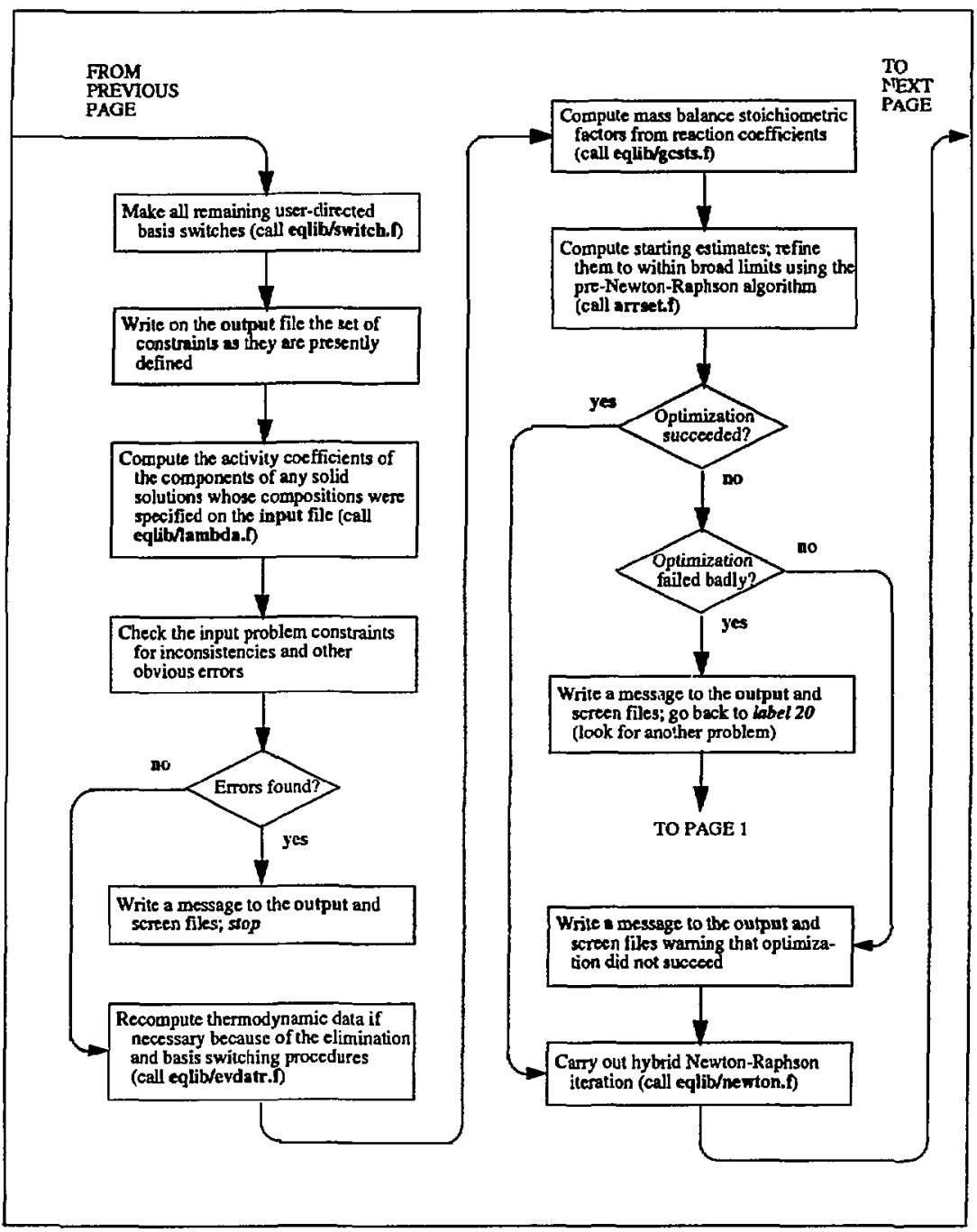

Figure 4 (continued, page 3 of 4). Simplified flow diagram of the EQ3NR main program (eq3nr.f). 


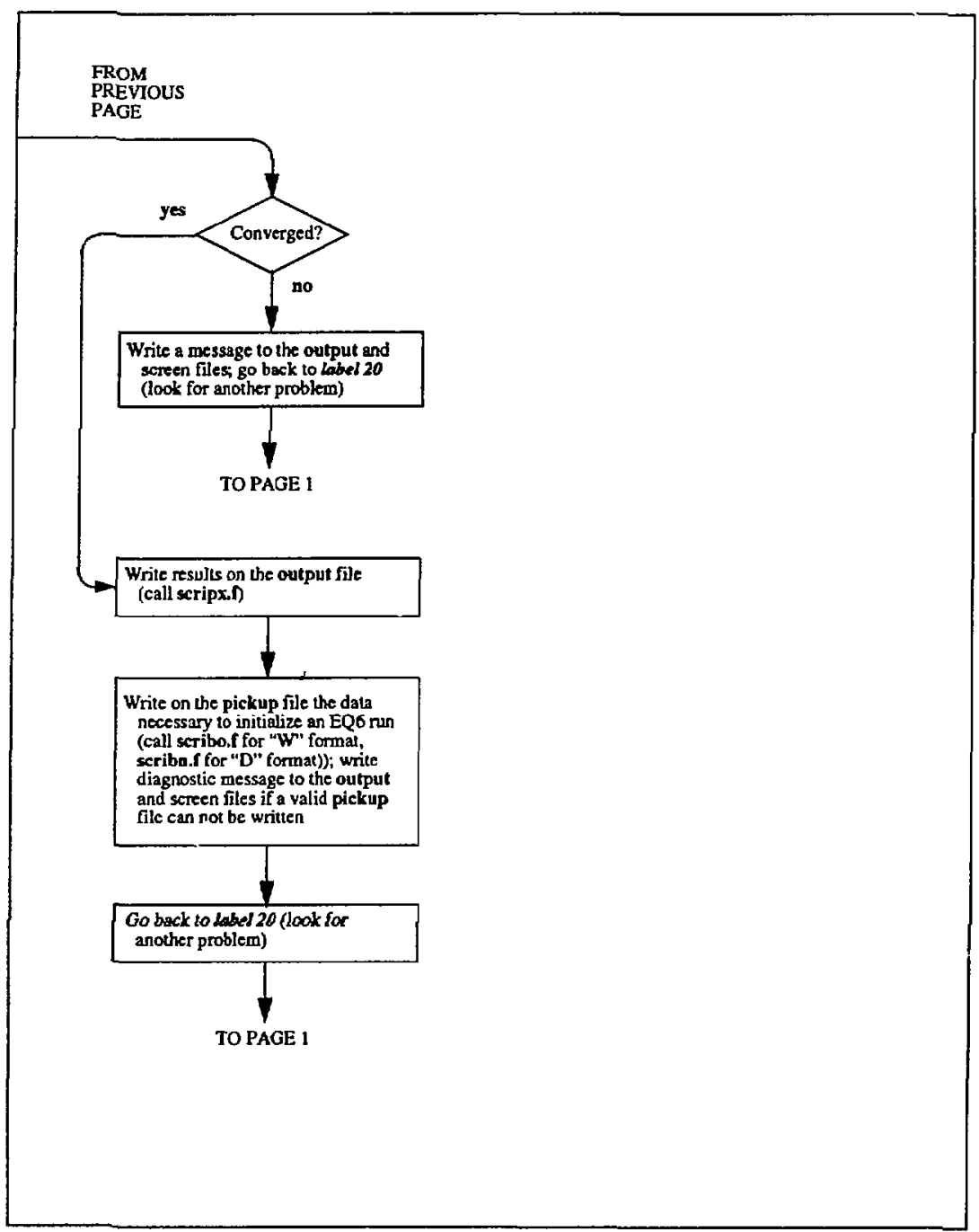

Figure 4 (continued, page 4 of 4). Simplified flow diagram of the EQ3NR main program (eq3nr.f). 
If the input file is in "W" format, module rdinp.f calls module readx.f to read the next problem. If it is in " $\mathrm{D}$ " format, it calls module rdninp.f to oversee the reading of the next problem. Unlike readx.f, rdninp.f carries out its function by calling a fairly large number of other modules, all of which have names beginning with "rd" or "get." Calls to either readx.f or rdinp.f result in the writing of an instant echo of the input file to the output file. If no problem is found at the current position of the file pointer, the main program is notified and it then proceeds as described above to make a normal exit.

If an input problem description is found on the input file, eq3nr.f sets the temperature range flag ninr calling the EQLIB module gntpr.f. It then gets the name of the chosen activity coeffi$\therefore$ tion corresponding to the iopgl activity coefficient option switch. This is a descriptive c.. : ier string that will be used subsequently. The code then calls module indatx, $f$ to read the supporting data file, datal. This module checks a flag contained on the data fle to insure that the kind of data file provided is consistent with the activity coefficient option selected on the input file. If it is sot, this module writes an error message to the screen and output files and execution stops. Otherwise, it proceeds to read the standard state thermodynamic data on the data file. All basis species are loaded into memory (even if they are not needed). All other types of species are loaded into memory only if they are relevant to the current problem.

The primary method of indexing data pertinent to species, reactions, etc., follows from the reading of the data file. Correspunding data read from the input file are stored in holding anrays. For example, the names of the basis species to include in the model and the corresponding "csp" inputs are initially stored in ho'ding arrays called uspecb and cspb, respectively. The names of the aqueous species read from the data file are kept in the uspec array. The uctual csp array is constructed by name matching between the uspecb and uspec arrays. This is done by the module gesp.f, which is called by indatx.f. The module indatx.f also calls the EQL IB module alters.f to execute any nxmod "alter" options that may have been included on the input file.

The main program then calls the EQLIB module gspion.f to find the indices of the hydrogen and chloride ions, and follows this by calling the module figstx.f to set the species status flags. The latter module may in turn call the EQLIB module supprs.f to execute any nxmod "suppress" options that may have been included on the input file.

Execution then returns to the main program. If iopg $1 \leq 0$, ea3nr.f calls the module inbdot.f to read from the data file the hard core diameter (azero) and insgfl flag data required for the B-dot activity coefficient model. This is done even if iopgl $=-1$ (the Davies equation option, in which case these data are not used). If jopgl $=1$, eq3nr.f calls the module inupt.f to read from the data file the relevant interaction parameters needed for Pitzer's equations. This is followed by calls to the EQLIB modules bdslx.f and bdmlx.f, which build index arrays used in the evaluation of Pitzer's equations. Only the data needed for the current problem are loaded into memory by either inhdot.f or inupt.f.

The thermodynamic data that have been read in at this point are in various forms which do not generally correspond to the data reguired at the temperature for the yiven problem. For example, the equilibrium constants as read from the data file are only represented as the coefficients of interpolating polynomials. The main program then calls the EQL.1B module evdata.f to compute the needed data for the temperature specified for the current problem. 
The main program then checks the redox constraints for the current problem to see if there are any conficts. If any are found, it resolves them and writes appropriate warning messages to the screen and output files. It then sets default values as required for some of the input file parameters. The next action of the main program is to call module echox.f. This writes an echo of the current problem to the output file. This echo includes the values of any default parameters that have been assigned.

The next action of eq3nr.f is to call module setup.f. This converts concentration data which are not in terms of molalities to molalities. The main program then identifies the species to be adjusted for electrical balance, or finds one which is suitable for this purpose if that is what the user requested on the input file. A choice made by the user is then checked for suitability. Some warnings and notes may be written to the screen and output files if any problems are apparent, such as the specified species not having any electrical charge (in which case the calculation may well fail, but not necessarily, as the concentrations of charged non-basis species will change in response to adjustments to the concentration of the species defined in the balance constraint).

The main program then executes those basis switches that were specifed on the input file and involve switching the roles of strict and auxiliary basis species (other basis switches specified on the input file are done laier). In doing this, it makes use of the EQLIB module switch.f. Then it writes a list of inactive species to the output file. These include species which have been suppressed by user options on the input file and species which were loaded from the data file, but for which the requisite thermodynamic data are lacking.

Module eq3nr.f then identifes the auxiliary basis species which defines the redox couple to be used to calculate the default redox state, if this option has been selected on the input file. It then checks to insure that there are no cases of a jflag value of 30 being assigned to a strict basis species. It then salls the EQLlB module elim.f to eliminate all auxiliary basis species with jflag = 30 from the active basis set. Then it executes (again by calling the EQLIB module switch.f) those basis switches which were specified on the input file and bring non-basis species into the basis set. The main program then writes to the output file a table describing the input constraints as it presently understands them. If solid solution compositions have been entered on the input file, the main program now calls the EQLIB module lambda.f to evaluaie the activity coefficients of the components of these phases.

The main program then makes a rather extensive set of tests on the current problem inputs, looking for inconsistencies and other obvious errors. If problems are found, error messages are written to the screen and output files. At the end of this process, if one or more errors have been detected, the code stops execution.

The next action of eq3nr.f is to call the EQLIB module evdatr.f to recor pute those thermodynamic data which have to be changed in response to the rewriting of reactions due to elimination of auxiliary basis species from the active basis set and basis switching. During the actual rewriting of reactions associated with such activities, the associated interpolating polynomials are recalculated, but the corresponding eguilibrium constants are not immediately recalculated. The equilibrium constants that were evaluated previously by evdata, $\mathrm{f}$ (which itself called evdatr.f to do this) are presently not used in any code function. In the past, they have been used to verify that the data were being properly reconstructed in the code prior to the steps in which the reactions 
were rewritten. Depending on the choice of the input file option switch iopr 2 , the reactions and associated data may be written to the output file after these actions have taken place. Module eq3nr.f then calls the EQLIB module gests.f, which computes the stoichiometric mass balance factors, which are kept in the storl array.

At this point, the code is ready to solve the system of algebraic equations posed by the current problem. The main program calls module arrset.f, which sets up the appropriate computational structures (e.g., the array defining the master iteration variables), assigns starting values, and attempts to refine them using the pre-Newton-Raphson optimization algorithm discussed in Chapter 9. The flow of execution in this module is illustrated in the simplified flow diagram in Figure 5. Note that there is an initial estimation procedure, followed by a fairly complex optimization process. This consists of an outer loop structure (loops) in which automatic basis switching is carried out (this happens only if iopt 2 is set to 1 on the input file). Inside this is a middle loop structure (passes) in which $\Sigma m, I$, and the activity coefficients of aqueous species are updated. Inside this in tum is an inner loop (cycles) in which the primary optimization (adjustment of concentrations of the basis species) is carried out. In applying the optimization algorithm, arrset.f calls module arrsim.f to compute refined values for those basis species concentrations which must be determined simultaneously (instead of by successively evaluating individual equations for the basis species concentrations, as is the case in the rest of the algorithm).

The goal of the optimization procedure in arrset.f is only to get the iterated values to within about an order of magnitude of the solution, so that subsequent Newton-Raphson iteration will have sufficiently good values to start with.This optimization process may succeed or not. If no:, this module checks to see if any computed basis species concentrations are outrageously high. If this is the case, the problem as stated is almost assuredly ill-posed (has no realistic solution).

If the problem appears to be ill-posed, eq3nr.f writes a note to the screen and output files and gives up on the current problem and loops back to see if there is another problem on the input file. Otherwise, whether or not the optimization step succeeded, it continues by calling the EQLIB module newton.f to compute the final solution. This executes the hybrid Newton-Raphson algorithm that was also discussed in Chapter 9. The flow of execution in newton.f is illustrated in the simplified flow diagram in Figure 6 . This module in tum calls the module betas.f and matrix.f to compute, respectively, the residual functions and the Jacobian matrix. These are known to newton.f as "hetae" and "matrxe," respectively. Module newton.f also calls the module ncmpx.f to expand the system. This routine is known to it as "ncmpe." Module newton.f calls the EQLIB module ngcadv.f to recompute the activity coefficients. It also calls the EQLIB module nrstep.f to execute a single Newton-Raphson step. The flow of execution in nrstep.f is illustrated in the simplified flow diagram in Figure 7. Note that this is actually a fairly complex piece of coding.

If hybrid Newton-Raphson iteration fails, the main program writes an error message to the screen and output files. It then calls module ndiagx.f to examine the results of the failed calculation in an attempt to generate useful diagnostics. If any are generated, they are written to the screen and output files. Most of the possible messages that might be written identify the relevant aspects of ill-posed input. The code then loops back to look for another problem on the input file. 
If hybrid Newton-Raphson iteration succeeds, the code continues processing the current problem. The next action of the main program is to call the module scripx.f. This writes a description of all relevant results to the output file. As necessary, it calculates various secondary parameters before writing them. What this module writes is essentially all of what the user sees as the results of the calculation.

The next action of eq3nr.f is to see if a valid pickup file can be written. To be valid for transmittal to EQ6, the aqueous solution model must include each strict basis species which is linked to each auxiliary basis species in the model. If a valid pickup file can not be written, the main program writes a note to the screen and output n̂les. Otherwise, a pickup file is then written. If the input file was in "W" format, the main program calls the module scribo.f, which writes the pickup file in the corresponding format. If the input file was in " $D$ " format, it calls the module scribx.f, which writes the pickup file in the corresponding format.

This terminates all code activity on the current problem. The main program then loops back to look for another problem on the input tile. 


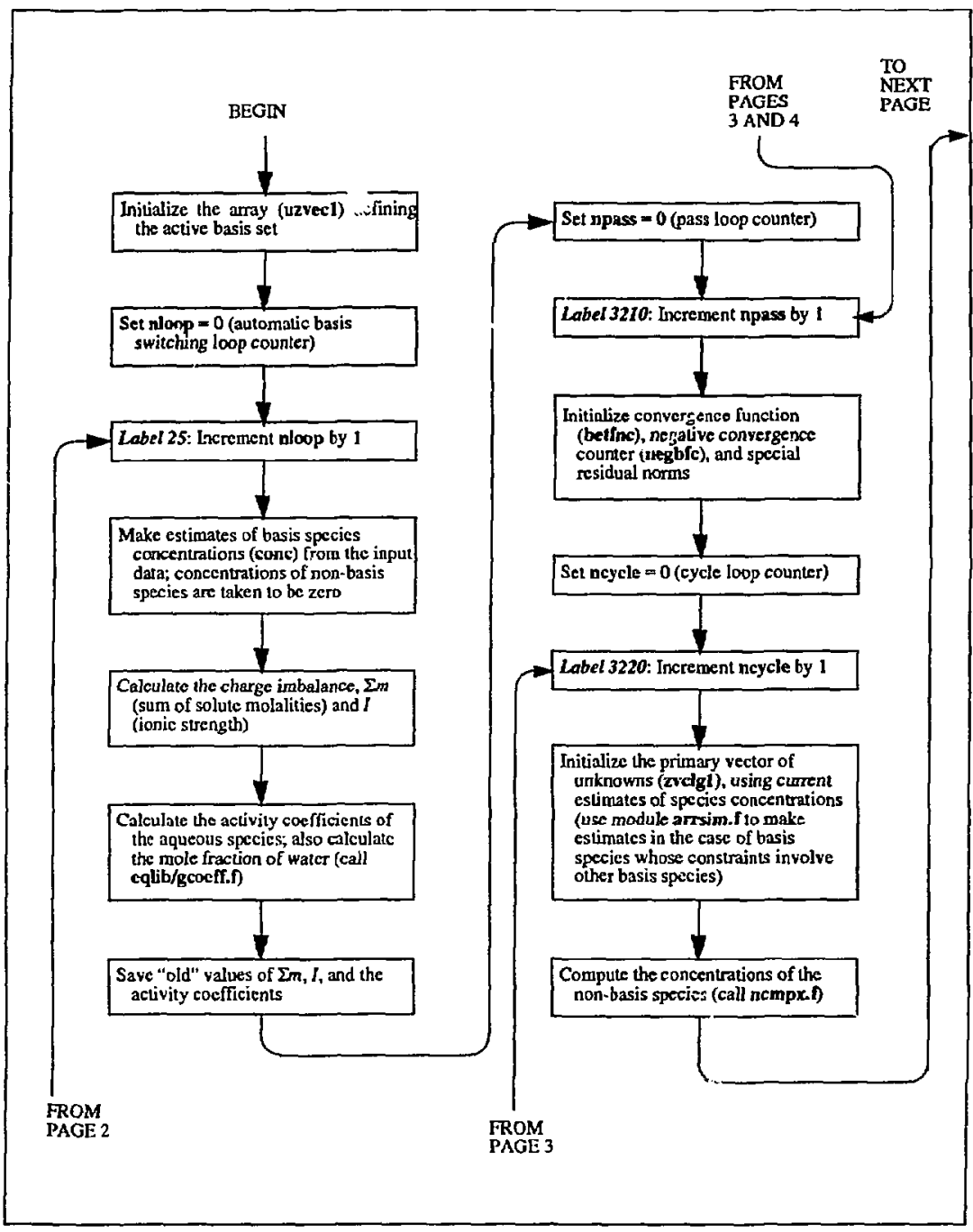

Figure 5 (page 1 or 4). Simplified flow diagram for creation of starting valtes and pre-Newton-Raphson optimization (from the viewpoint of module arrsetn). 


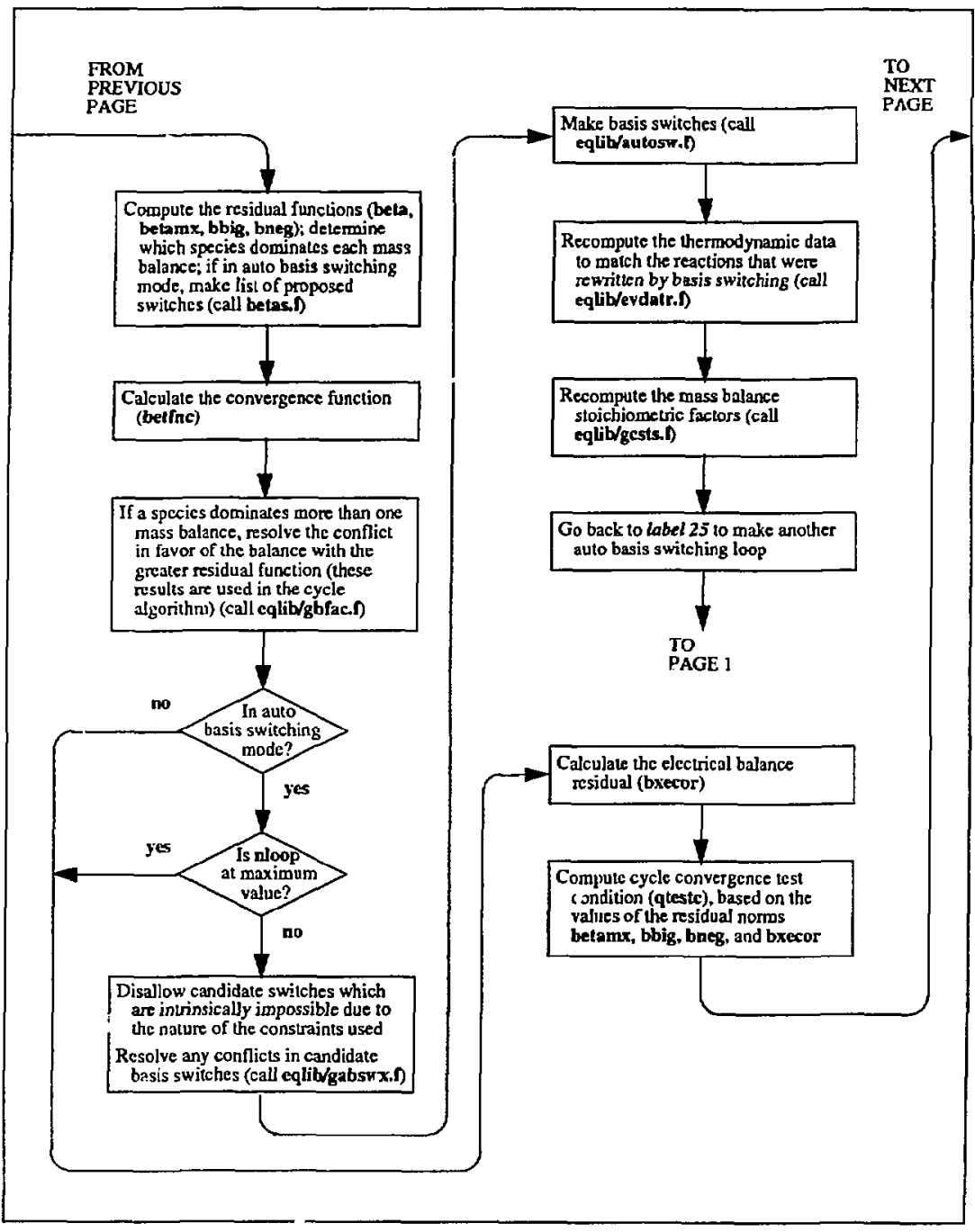

Figure 5 (continued, page 2 of 4). Simplified flow diagram for creation of starting values and pre-Newton-Raphson optimization (from the vicwpoint of modlulc arrset. I). 


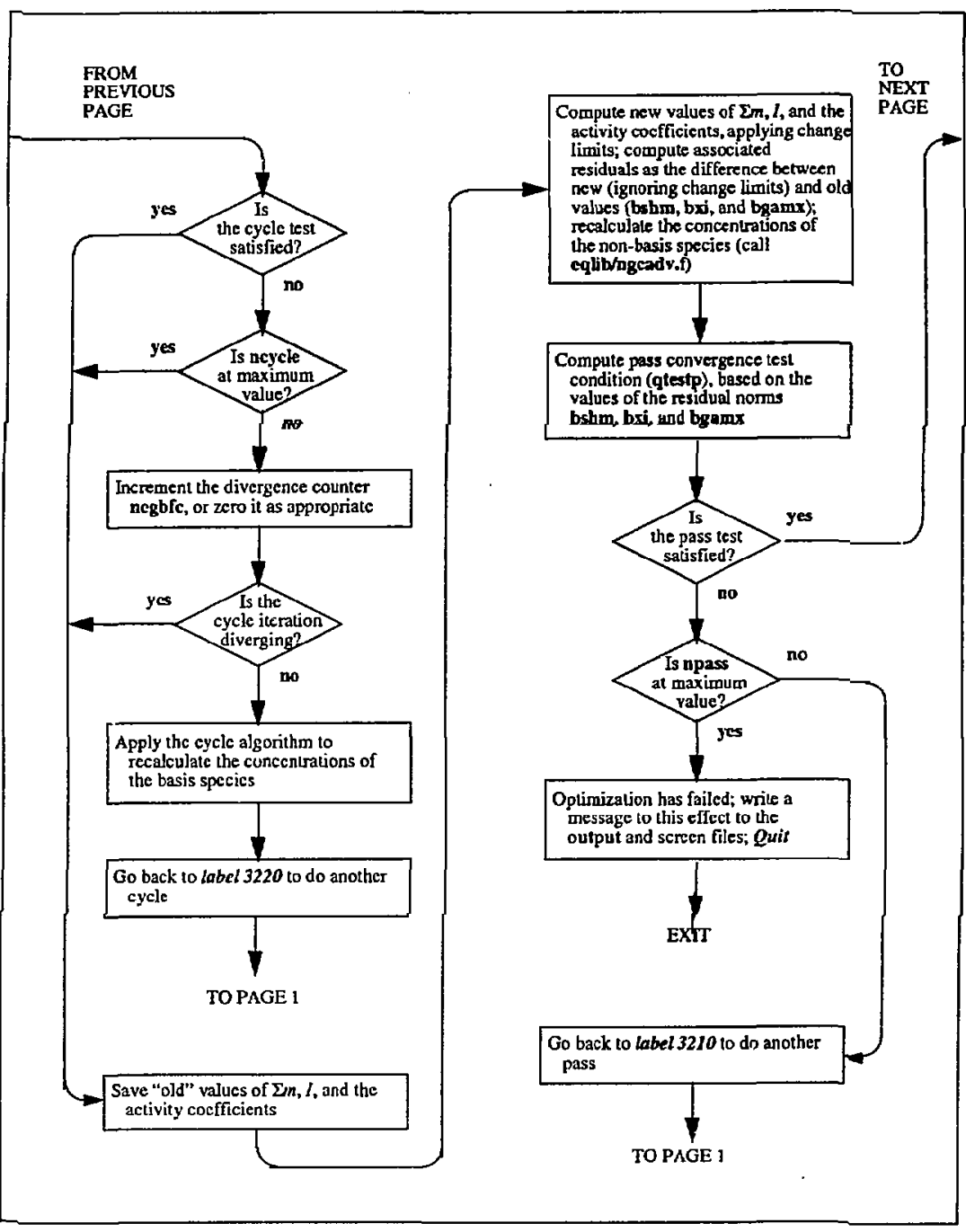

Figure 5 (continued, page 3 or 4). Simplified now diagram for creation of starting values and pre-Newton-Raphson optinization (from the viewpoint of module arrset.f). 


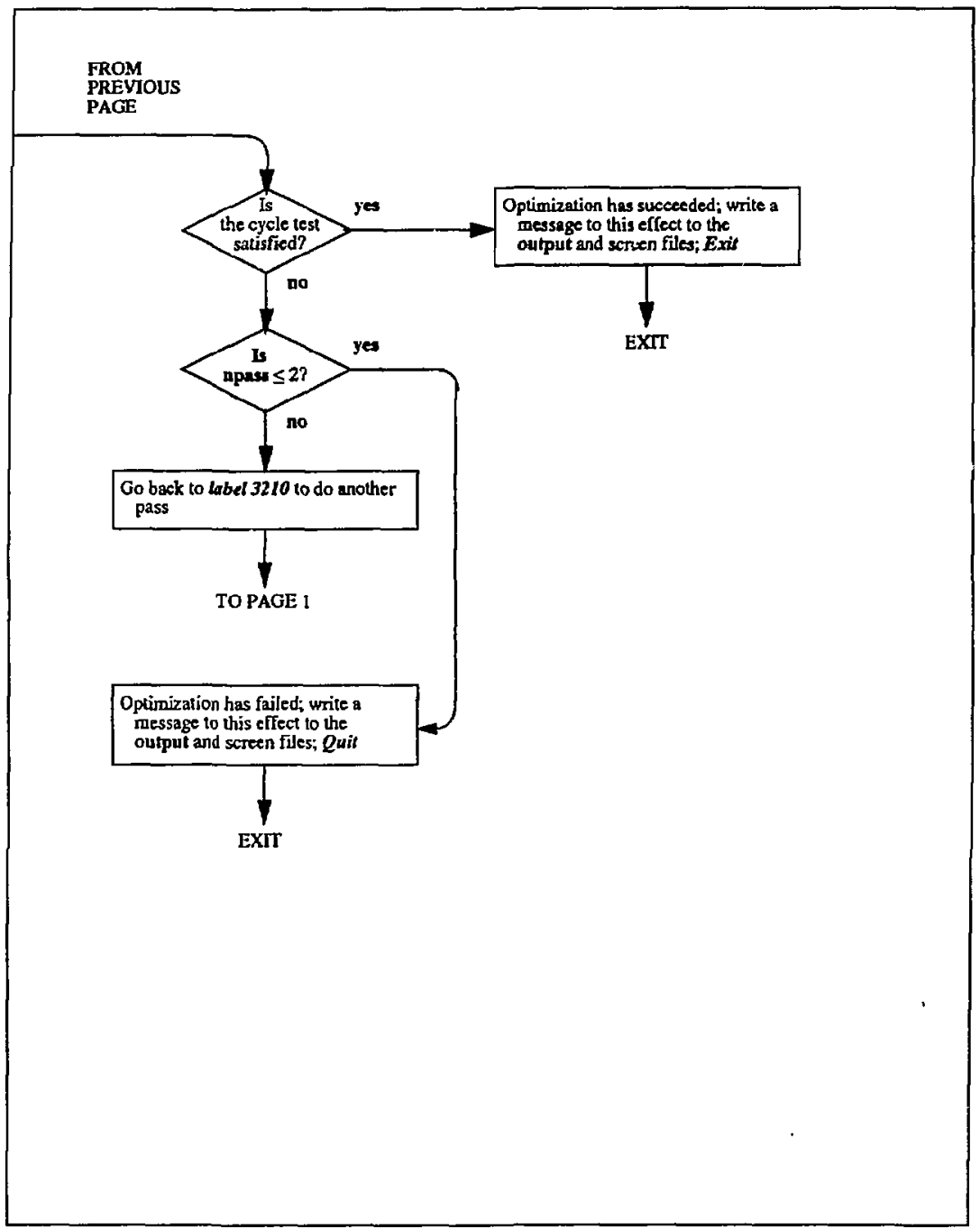

Figue 5 (conlinued, page 4 of 4). Simplified flow diagram for creation of slarting values asd pre-Newton-Raphson optimization (from the viewpoint of nodule arrset.). 


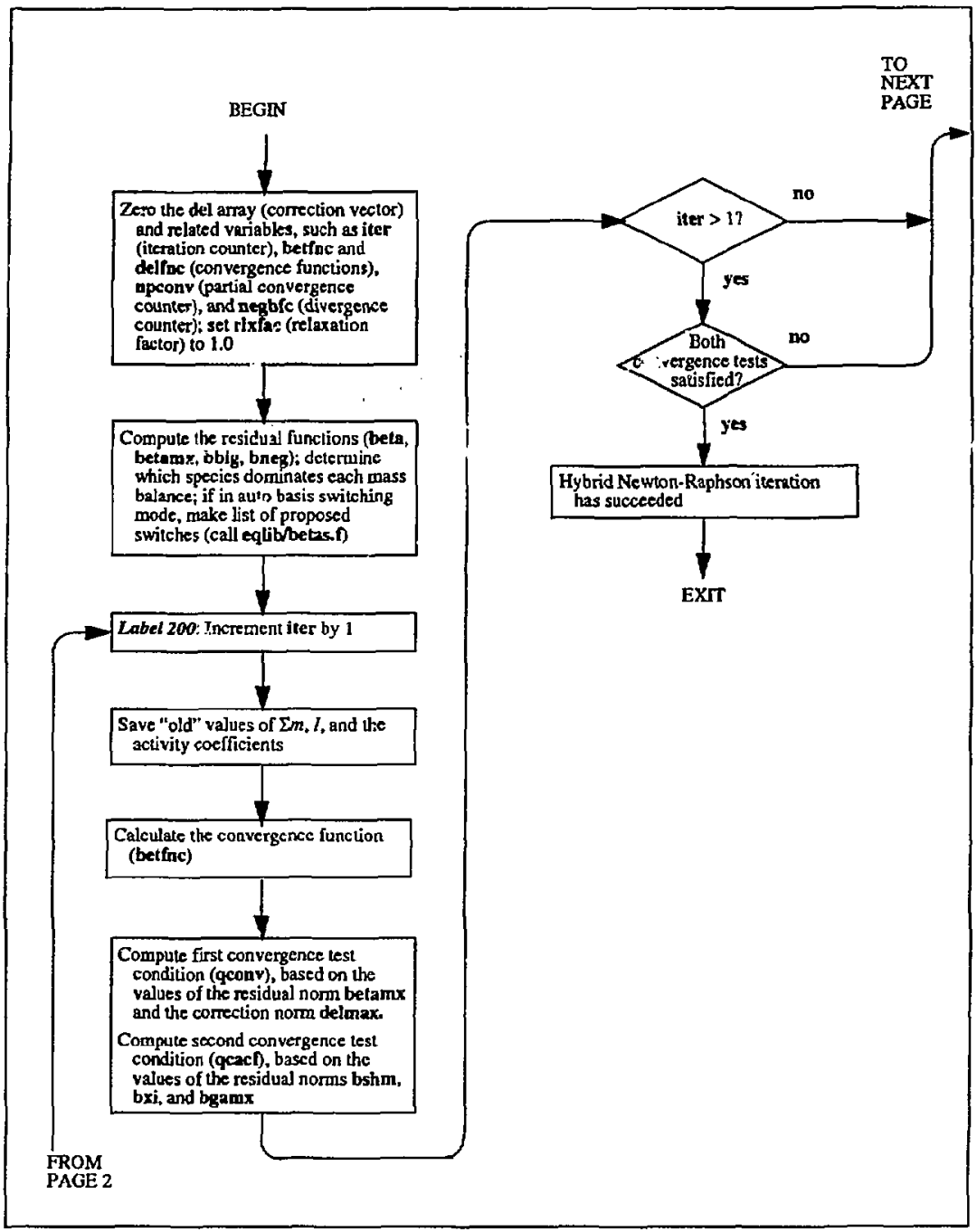

Figure 6 (page 1 of 2). Simplified flow diagram for hybrid Newton-Raphsson iteration (from the viewpoint of the EQLIB module newton.f). 


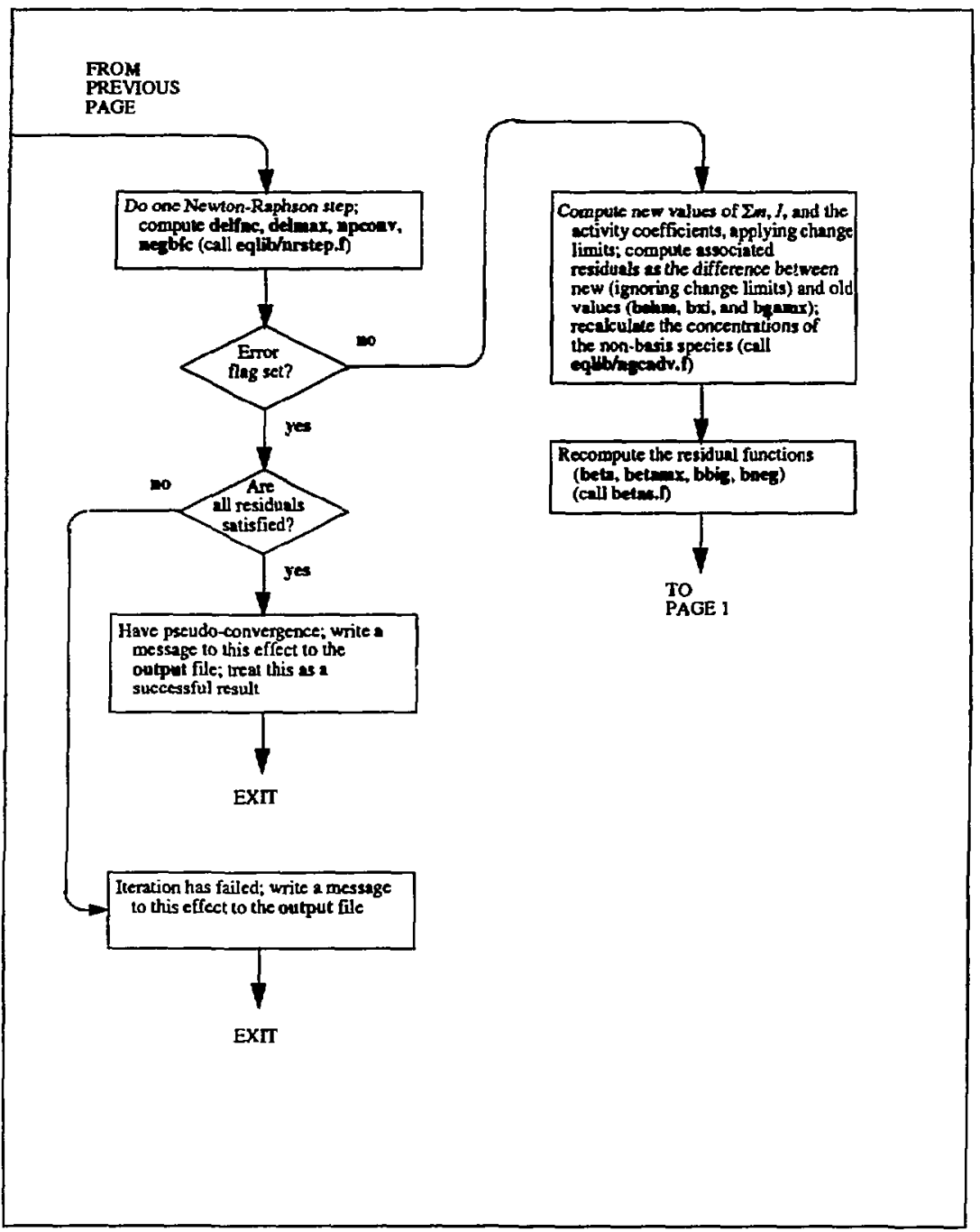

Figure 6 (continued, page 2 of 2). Simplified flow diagram for hybrid Newton-Raphson itcration (from the viewpoint of the EQLIB module newton.f). 


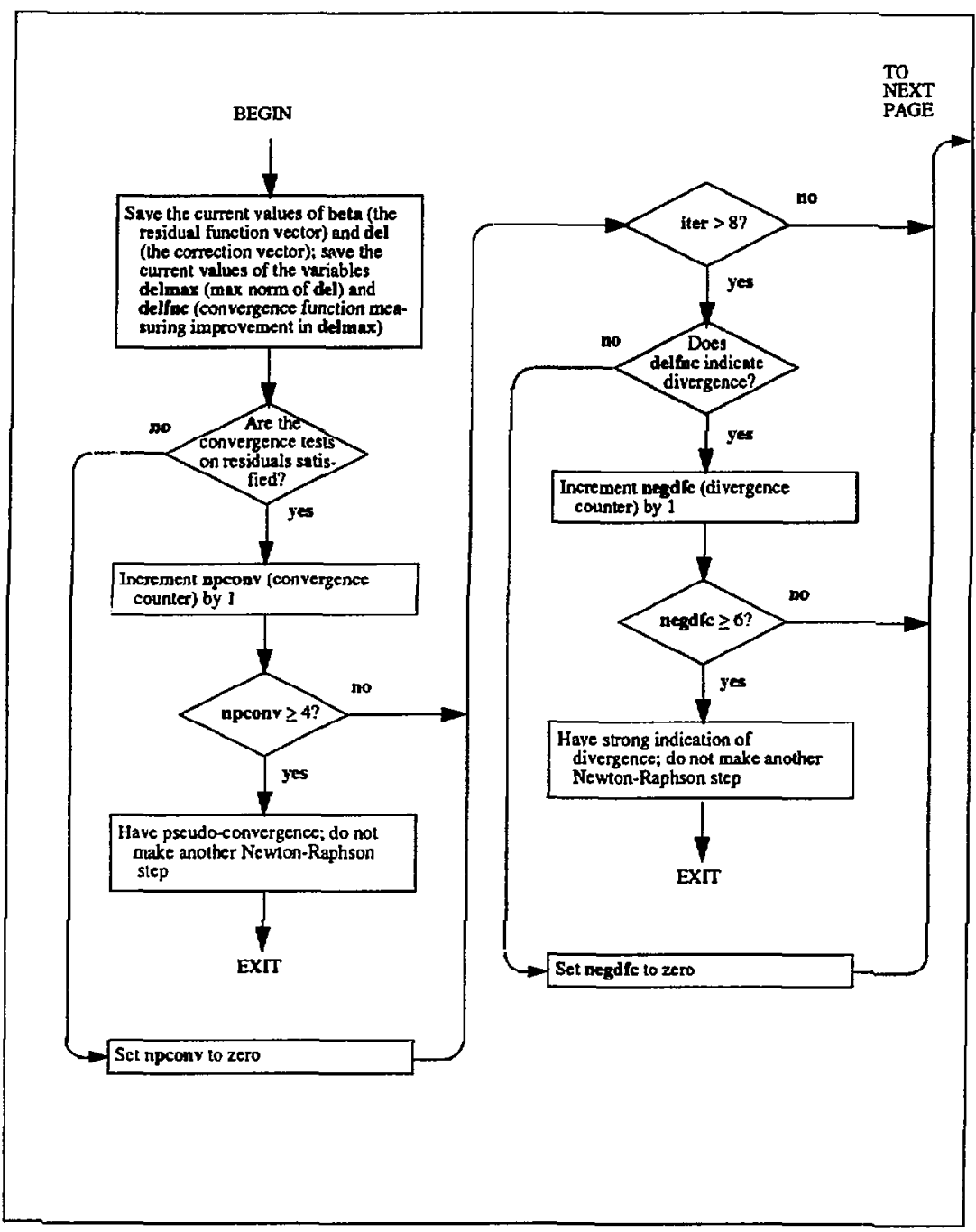

Figure 6 (page 1 of 4). Sirrplified flow diagram for a Newton-Raphson step (from the viewpoint of the EQLIB module nrstep. $)$. 


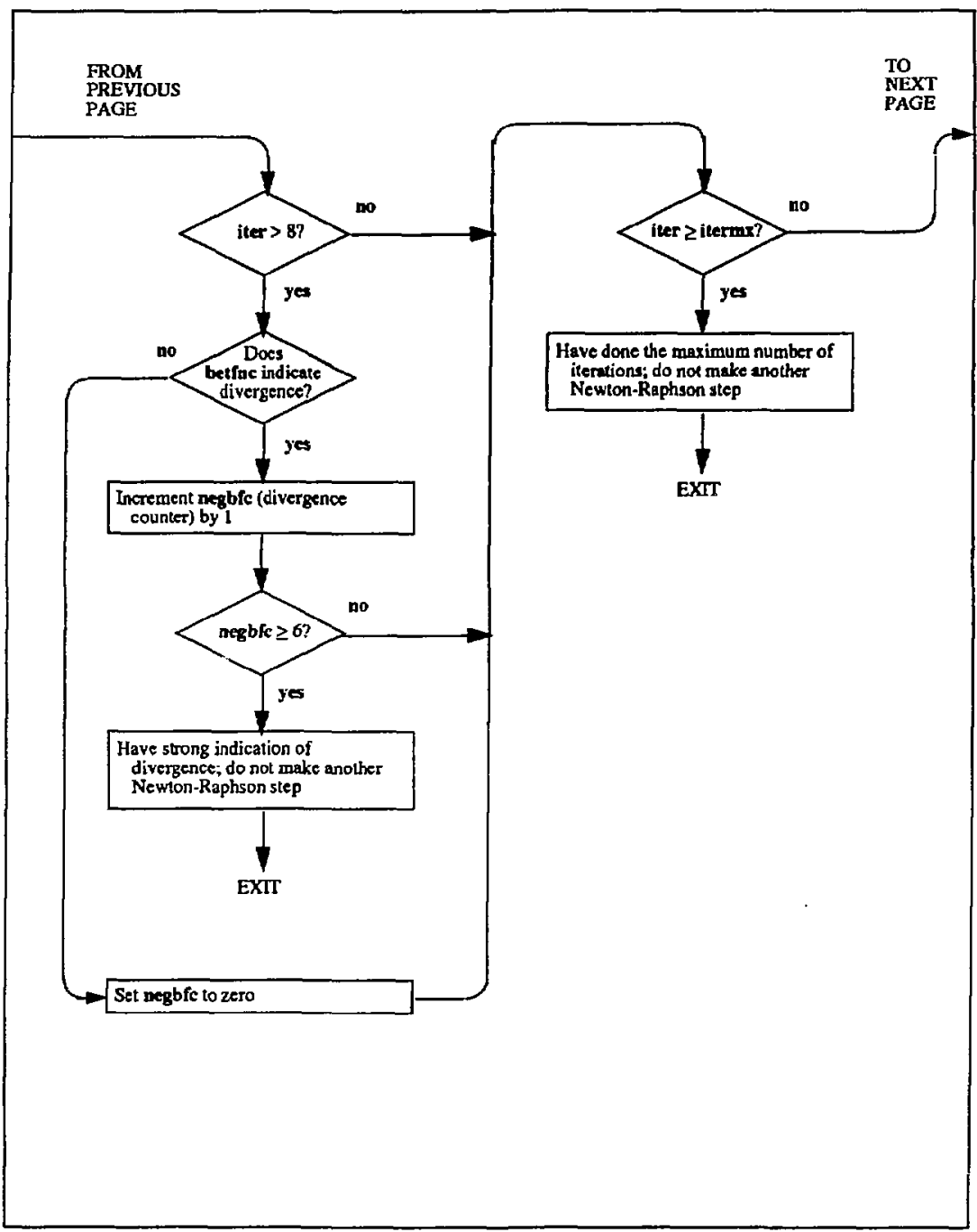

Figure 6 (page 2 of 4). Simplified how diagram for a Neviou-Raphson step (from the viewpoint of the EQLIB modulc nrstep.f). 


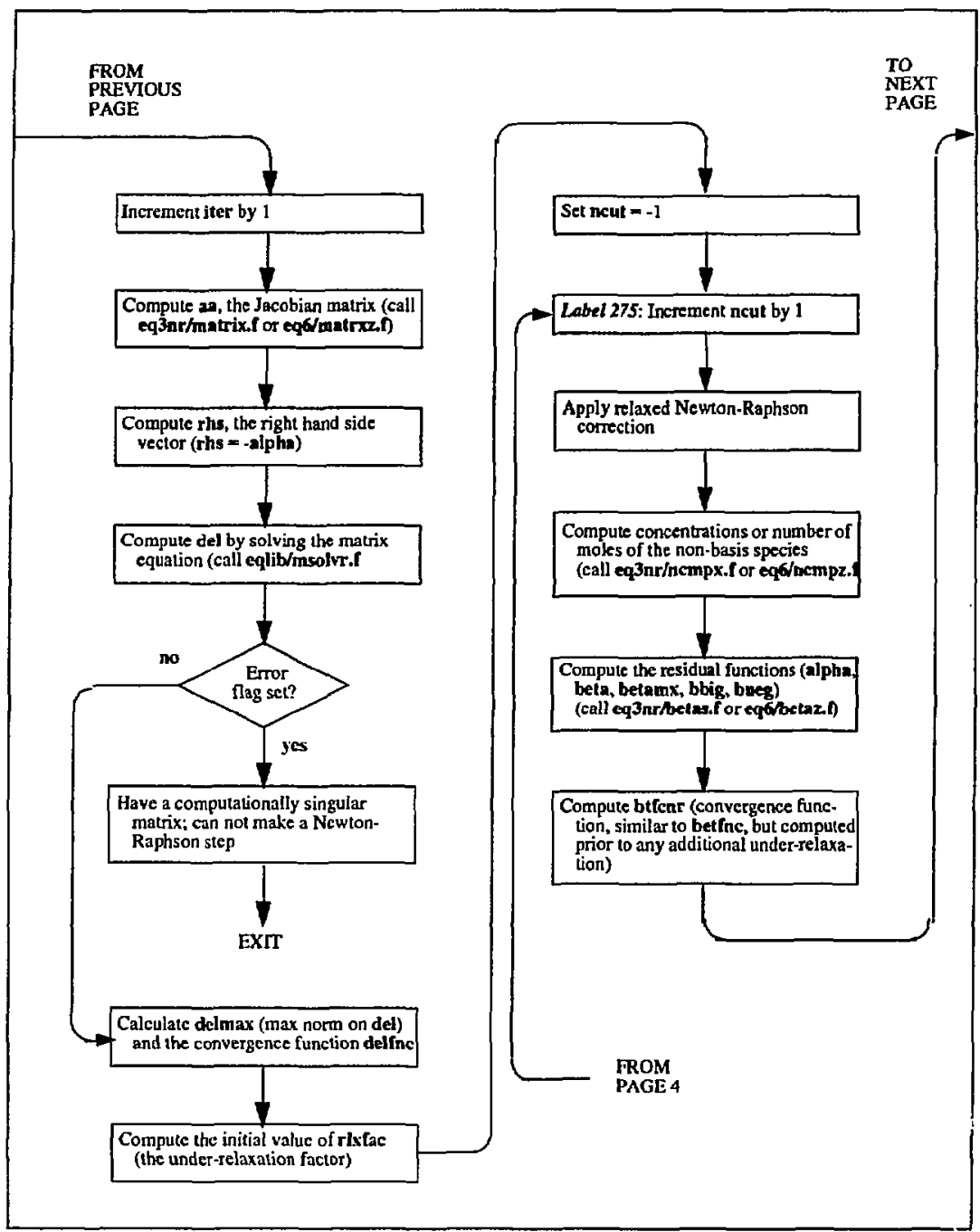

Figure 7 (page 3 of 4). Simplificd flow diagrant for a Newton-Raptsson siep (from the viewpoint of the EQLIB module nrstep.i). 


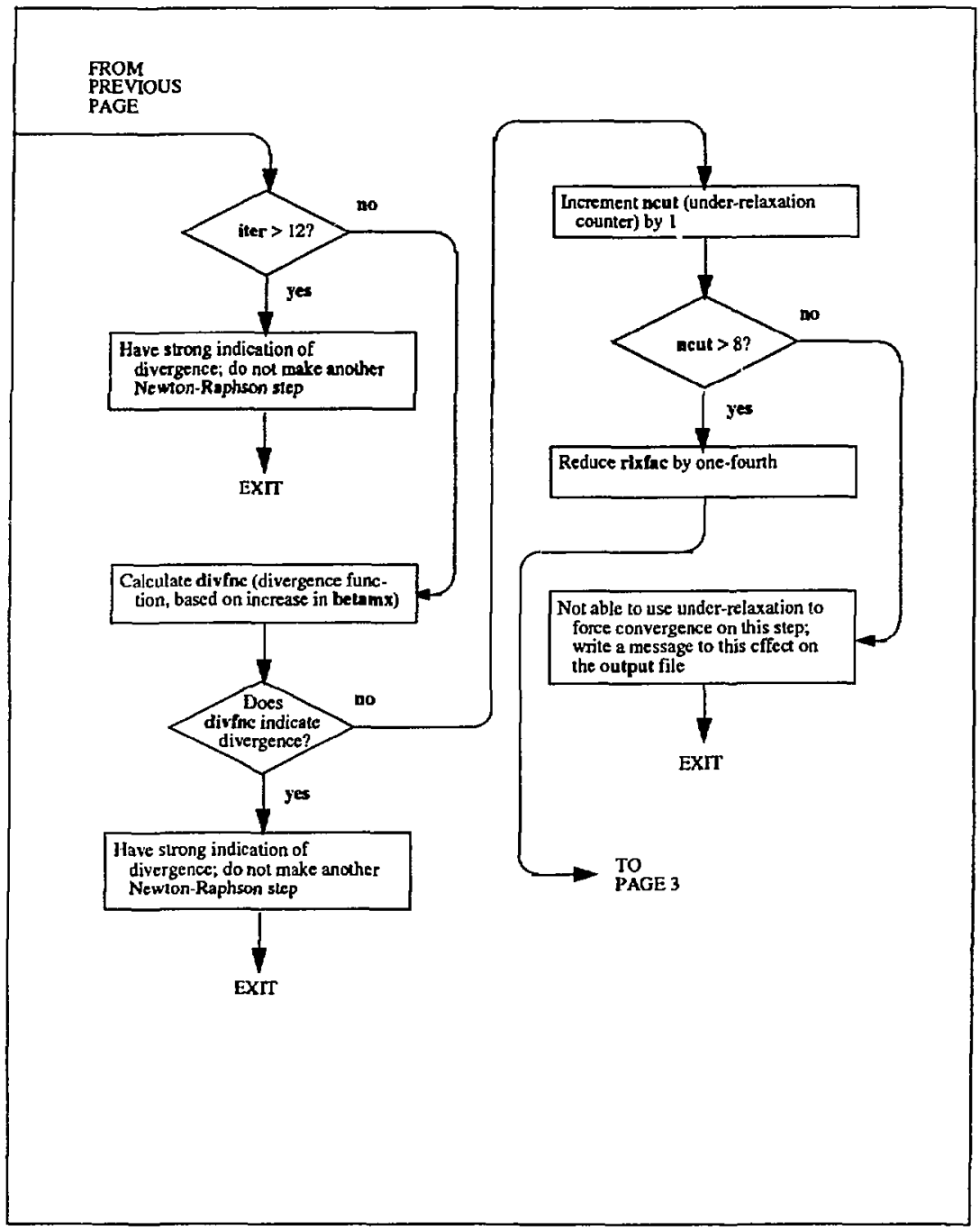

Figure 7 (page 4 of 4). Simplified flow diagram for a Newton-Raphson step (from the viewpoint of the EQLIB module nrstep. $)$. 


\section{Acknowledgments}

Thanks are due to many individuals for their contributions in one form or another to the development of EQ3/6. Among these are Roger Aines, Carol Bruton, Bill Bourcier, Manny Clinnick, Paul Cloke, Stephanie Daveler, Joan Delany, Don Emerson, Mandy Goldner, Bob Herrick, Dana Isherwood, Ken Jackson, Jim Johnson, Suzanne Lundeen, Bill McKenzie, Judith Moody, Miki Moore, Ignasi Puigdomenech, Larry Ramspott, Terry Steinborn, and Brian Viani. I thank Brian Viani for his technical review of this report, which led to a number of improvements. I also thank Jim Blink for his comments on the manuscript. 


\section{References}

Ananthaswamy J., and Atkinson, G., 1984, Themnodynamics of concentrated electrolyte mixtures. 4. Pitzer-DebyeHückel limiting slopes for water from 0 to $100^{\circ} \mathrm{C}$ and from $1 \mathrm{~atm}$ to $1 \mathrm{kbar}$. Journal of Chemical and Engineerjig Data, v. 29, p. $81-87$.

Archer, D. G., 1990, Effect of revisions of Debye-Hückel limiting law coefficients on the thermodynamic paramcters for strong-electrolyte solutions: Journal of Chemical and Engineering Data, v. 35, p. 340-344.

Baes, C. F, Jr., and Mesmer, R. E., 1976, The Hydrolysis of Cations: Wiley Interscience, New York.

Ball, J. W., Jenne, E. A., and Nordstrom, D. K., 1979, WATEQ2: A computerized chemical model for trace and major clement speciation and mineral equilibria for natural water, in Jenne, E. A., cditor, Chemical Modeling in Aqueous Systems, American Chemical Society Symposium Scries, y. 93, American Chemical Sociely, Washington, D. C., p. 815-835.

Barta, L., and Bradley, D. J., 1985, Extension of the specific interaction model to include gas solubilities in high temperature brines: Geochimica et Cosmochimica Acta, v. 49, p. 195-203.

Bassett, R. L., and Melchior, D.C., 1990, Chemical modeling of aqueous systems: An overview, in Melchior, D. C., and Bassett, R. L., editors. Chemical Modeling of Aqueous Systems II, American Chemical Society Symposiun Series, v. 416, American Chemical Society, Washington, D. C., p. 1-14.

Bates, R. G., 1964, determination of pH: Wilcy and Sons, New York.

Bates, R. G., 1973, Ion activity scales for use with ion-selective electrodes: Pure and Applied Chemistry, v. 36, p. 407-420.

Bates, R. G., and Alfenaar, M., 1969, Activity standards for ion-selective electrodes, in Durst, R. A., cditor, IonSelective Electrodes, Proccedings of an NBS Symposium, NBS Special Publication 314, p. 191-214.

Bates, R. G., and Robinson, R. A., 1974, An approach to conventional scales of ionic activity for the standardization of ion-sclective clectrodes: Pure and Applied Chemistry. v. 37, p. 575-577.

Ben-Yaakov, S., and Sass, E., 1977, Independent estimatc of the pH of Dead Sea brine: Limnology and Oceanography, v. 22, p. 374-376.

Besner, R. A., 1971, Principles of Chemical Sedimentology: McGraw-Hill, New York.

Bourcier, W. L., 1985, Improvements in the Solid Solution Modeling Capabilities of the EQ3/6 Geochemical Code: UCID-20587, Lawsence Livernore National Labnratory, Livermore, Califomia.

Bourcier, W. L., 1989, Improvements in the solid solution modeling capabilities of EQ3/6, in Jackson, K. J., and Bourcier, W. L, editors. Proceedings of the Workshop on Gcochemical Modeling, CONF-8609134, Lawtence Livermore National Laboratory, Livermore, Califomia p. 41-48.

Busenberg, E., 1978, The products of the interaction of feldspars with aq̣ueous solutions at $25^{\circ} \mathrm{C}$. Gcochimica et Cosmochimica Acts, 1.42, p. 1679-1686.

Camahan, B., Luther, H. A., and Wilkes. J. O., 1969. Applied Numerical Methods: John Wiley \& Sones, New York.

Carothers, W. W., and Kharaka, Y. K., 1978, Aliphatic acid anions in oil-field waters- Implications for origin of natural gas: The American Association of Pelrolcum Gcologists Bulletin, v. 62, p. 2441-2453. 
Chen, C.-T, A., and Marsball, W. L., 1981 , Amorpbous silica solubilities IV, Bebavior in pure water and aqueous sodium chloride, sodium sulfate. magnesium chloride, and magnesium sulfate solutions up $10350^{\circ} \mathrm{C}$ : Geochimica et Cosmochimica Acta, v. 46, p. 279-287.

Clegg, S. Lo, and Brimblecombe, P., 1989, Solubility of ammonia in pune aqueous and multicomponent solutions: Jourual of Physical Chemistry, v. 93, p. 7237.7248.

Clegg, S. L, and Brimblecombe, P., 1990, Solubility of volatilt electrolytes in multicomponent solutions with atmospheric applications, in Melchior, D. C., and Bassett, R. L., editors. Chemical Modeling of Aqueous Systems II, American Chemical Society Symposium Series, v. 416, American Chemical Society, Washington, D. C., p. 58-73.

Connaughton, L. M., Millero, F. J., and Pitzer, K. S., 1989, Volume changes for mixing the major sea salts: equations valid to ionic strength 3.0 and temperature $95^{\circ} \mathrm{C}$ : Journal of Solution Chemistry, v. 18, p. 1007-1017.

Crerar, D. A., 1975, A method for computing multicomponent equilibria based on equilibrium constants. Geochimica et Cosmochimica Acta, y, 39, p. 1375-1384.

Daveler, S. A., and Wolery. T. J., 1992, EQPT, A Data File Preprocessor for the EQ3/6 Software Package: User's Guide and Related Documentation (Version 7.0): UCRL-MA-110662-PT-II, Lawrence Livermore National Laboratory, Livermore, Califormia.

Davies, C. W., 1962, Ion Association: Butterwortlss, London.

Delany, J. M., and Lundeen, S. R., 1991, The LLNL Themochemical Data Base-Revised Data and File Format for the EQ3/6 Package: UCID-21658, Lawrence Livemore National Laboratory, Livermore, Califomia.

Drummond, S. E.,Jr., 1981, Boiling and Mixing of Hydrothermal Fluids: Chemical Effects on Mineral Precipitation: Ph.D. thesis, The Pennsylvania State University, University Park, Pennsylvania.

Felmy, A. R., and Weare, J. H., 1986, The prediction of borate mineral equilibria in natural waters: Application to Searles Lake, California: Geochimica et Cosmochimica Acta, v. 50, p. 2771-2783.

Freeze, R. A., and Cherry, J. A., 1979. Groundwater: Prentice-Hall, Inc., Englewood Cliffs, New Jersey.

Garrels, R. M., and Cbrist, C. L., 1965, Solutions, Minerals and Equilibria: Freeman, Cooper and Company, San Francisco.

Garrels, R. M., and Thompson, M. E., 1962, A chemical model for sca water at $25^{\circ} \mathrm{C}$ and one atmosphere total pressure: American Joumal of Science, v. 260, p. 57-66.

Greenberg, J. P., and Moller, N., 1989, The prediction of nineral solubilities in natural waters: $A$ chemical equilibrium model for the $\mathrm{Na}-\mathrm{K}-\mathrm{Ca}-\mathrm{Cl}-\mathrm{SO}_{4}-\mathrm{H}_{2} \mathrm{O}$ system to high concentration from 0 to $250^{\circ} \mathrm{C}$ : Geochimica et $\mathrm{Cos}-$ mochinica Acta, v. 53, p. 2503-2518.

Grenthe, I., Lemire, R. J., Muller, A. B., Nguyen-Trung, C., and Wanner, H., 1989, NEA-TDB Chenical Themodynamics of Uranium, OECD-Nuclear Energy Agency, Saclay. France (drafi manuscript; NNA.900815.0013).

Grenthe, I., Fuger, J., Lemire, R. J., Muller, A. B., Nguyen-Trung, C., and Wanner, H., editors, 1992. Chemical Thermodynamics of Uranium: Elscvier Science Publishing Company, Now York.

Harned, H. S., and Owen, B. B., 1958, The Physical Chenistry of Electrolyte Solutions, 3rd Edition: ACS Monograph 137, Reinhold, New York. 
Harvie, C. E, 1981. Theoretical Investigations in Geochemistry and Atom Surface Scattering: Ph.D. thesis, University of California, San Diego.

Harvie, C. E, Meller, N., and Weare, J. H., 1984, The prediction of mineral solubilities in natural watcrs: the Na-K$\mathrm{Mg}-\mathrm{Ca}-\mathrm{H}-\mathrm{Cl}-\mathrm{SO}_{4}-\mathrm{OH}-\mathrm{HCO}_{3}-\mathrm{CO}_{3}-\mathrm{CO}_{2}-\mathrm{H}_{2} \mathrm{O}$ system to high ionic strengths at $25^{\circ} \mathrm{C}$ : Geochimica et Cosmochimica Acta, v. 48, p. 723-751.

Harvie, C. E., and Weare, J. H., 1980, The prediction of mineral solubilities in natural waters: the $\mathrm{Na}-\mathrm{K}-\mathrm{Mg}-\mathrm{Ca}-\mathrm{Cl}$ $\mathrm{SO}_{4}-\mathrm{H}_{2} \mathrm{O}$ system from zero to high concentrations at $25^{\circ} \mathrm{C}$. Geochimica et Cosmochimica Acta, y. 44, p. 981 . 997.

Helgeson, H. C., 1967, Thermodynamics of complex dissociation in aqueous solutions at elevated temperatures: Joumal of Physical Chemistry, v. 71, p. 3121-3136.

Helgeson, H. C., 1969, Themodynamics of hydrothermal systems at elevated temperatures and pressures: American Journal of Science, v. 267, p. 729-804.

Helgeson, H. C., Brown, T. H., Nigrini, A., and Jones, T. A., 1970, Calculation of mass transfer in geochemical processes involving aqueous solutions: Geochimica et Cosmochimica Acta, v. 34, p. 569-592-877.

Helgeson, H. C., Delaney, J. M., Nesbitt, H. W., and Bird, D. K., 1978, Summary and critique of the thermodynamic properties of rock-forming minerals: American Joumal of Science, v. 278-A, p. 1-229.

Helgeson, H. C., and Kirkham, D. H., 1974a, Theoretical prediction of the thenmodynamic behavior of aqueous electrolytes at high pressures and temperatures: I. Summary of the themodynamic/electrostatic properties of the solvent: Amcrican Joumal of Science, v. 274, p. 1089-1198.

Helgeson, H. C., and Kirkham, D. H., 1974b, Theoretical prediction of the thermodynamic behavior of aqueous electrolyles at higl pressures and temperatures: IL. Debye-Hückel parameters for activity coefficients and relative partial-molal properties: American Journal of Science, y. 274, p. 1199-1261.

Helgeson, H. C., and Kirkham, D. H., 1976, Theoretical prediction of the themodynamic behavior of aqueous electrolyes at high pressures and temperatures: IV. Calculation of activity coefficients, osmotic coefficients, and apparent molal and slandard and relative partial molal properties to $600^{\circ} \mathrm{C}$ and $5 \mathrm{~kb}$ : American Journal of Science. v. 281 , p. $1249-1516$.

Helgeson, H. C., Kirkham, D. H., and Flowers, G. C., 1981, Theoretical prediction of the themodynamic bethavior of aquecous electrolytes at high pressures and temperatures: 1Il. Equation of state for aqueous species at infinite dilution: American Joumal of Science, v. 276, p. 97-240.

Henley, R. W., Truesdell, A. H., Barton, P. B., Jr., and Whitncy, J. A., 1984, Fluid-Mineral Equilibria in Hydrothermal Systems: Keviews in Economic Geology, v. 1, Society of Economic Geologists, The Economic Geology Publishing Company, El Paso, Texas.

Holmes, H. F., and Mesmer, R. E., 1986a, Isopiestic studies of aqueous soletions at elevated temperatures VIll. The alkali-metal sulfates: Joumal of Chemical Thermodynamics, v. 18, p. 263-275.

Holmes, H. F., and Mesmer, R. E., 1986b, Themoxlynanics of ayjueous solutions of the alkali metal sultates: Journal of Solution Chenisitry, v. 15, p. 495-5I8.

Hosteter, J. D. 1984, Electrode electrons, atueous electrons, and redox potentials in natural waters: Ancrican Journal of Science, v. 284, p. 734-759. 
Huang, H.-H., 1989, Estimation of Pitzer's ion interaction parameters for electrolytes inyolved in complex formation using a chemical equilibrium model: Journal of Solution Chemistry, v. 18, p. 1069-1084.

INTERA Eavironmental Consultants, Inc., 1983, Geocbemical Models Suitable for Perfomance Assessment of Nuclear Waste Storage: Comparison of PHREEQE and EQ3/EQ6: ONWI-473, Office of Nuclear Waste Isolation, Battelle Project Management Division, Columbus, Obio.

Jackson, K. J., 1988, Verification and Validation Studies of the Addition of Pitzer's Equations to the EQ3/6 Brine Model: UCRL-53841, Lawrence Livermore National Laboratory, Livermore, California.

Jenne, E. A., 1981, Geocbemical Modeling: A Review: PNL,-3574, Battelle Pacific Northwest Laboratory, Richland. Washington.

Johnson, J. W., and Norton, D., 1991, Critical phenomena in hydrothermal systems: State, themodynamic, electrostatic, and transport properties of $\mathrm{H}_{2} \mathrm{O}$ in the critical region: American Joumal of Science, v. 291, p. 541-648.

Johnson, J. W., Oelkers, E. H., and Helgeson, H. C., 1992, SUPCRT92: A software package for calculating the standard molal thermodynamic properties of minerals, gases, aqueous species, and reactions from 1 to 5000 bars and $0^{\circ}$ to $1000^{\circ} \mathrm{C}$ : Computers and Geosciences, v. 18, p. 899-947.

Kharaka, Y. K., and Barnes, I., 1973, SOLMNEQ: Solution-Mineral Equilibrium Computations: United States Geological Survey Computer Contributions Publication 215-899.

Knauss, K. G., and Wolery, T. J., 1986, Dependence of albite dissolution kinetics on pH and time at $25^{\circ} \mathrm{C}$ and $70^{\circ} \mathrm{C}$ : Geochimica et Cosmochimica Acta, v. 50, p. 2481-2497.

Knauss, K. G., Wolery. T. J., and Jackson, K. J., 1990, A new approach to measuring pH in brines and other concentrated electrolytes: Geochimica el Cosmochimica Acta, v. 54, p. 1519-1523.

Knauss, K. G., Wolery, T. J., and Jackson, K. J., 1991, Reply to comment by R. E. Mesmer on "A new approach to measuring pH in brines and other concentrated electrolytes": Geochimica et Cosmochimica Acta, v. 55, p. 1177 1179.

Kodytek, V., and Dolejs, V., 1986, On the Pitzer equation for the osmotic coefficient of aqueous solutions of 3:1 rare earth clectrolytes; Zeitschrift für Physikalische Chemie (Leipzig), v. 267, p. 743-746,

Krauskopf, K., 1967, Introduction to Geochemistry: McGraw-Hill, New York.

Kumar, A., 1986, Prediction of densities of concentrated brines by Pitzer theory: Joumal of Chemical and Engineering Data, v. 31, p. 19-20.

Laxen, D. P. H, and Chandler, I. M., 1982, Comparison of filtration techniques for size distribution in freshwaters: Analytical Chemistry, v. 54, p. 1350-1355.

Langmuir, D., 1979, Techniques of estimating thermodynamic properties for some aqueous complexes of geochemical interest, in Jenne. E. A., editor, Chemical Modeling in Aqueous Systems, American Chemical Society Symposium Serics, v. 93, American Chemical Society, Washington, D. C., p. 353-387.

Lindberg, R. D., and Runnells, D. D., 1984, Ground water redox reactions: An analysis of equilibrium statc applied to Eh measurements and geochemical modeling: Science, v. 225, p. 925-927.

MacInnes, D. A., 1919, The activities of the ions of strong electrolytes: Joumal of the Chemical Society, v. 41, p. 1086-1092. 
Marcus, Y., 1977, The activities of potassium cbloride and of water in Dead Sea bribe: Geocbimica et Cosmochimica Acta, v. 41, p. 1739-1744.

Merino, E., 1979, Internal consistency of a wate! analysis and uncertainty of the calculated distribution of aqueous species at 25을 Geochimica et Cosmochimica Acta, v. 43, p. 1533-1542.

Mesmer, R. E., 1991, Comments on "A new approach to measuring pH in brines and other concentrated electrolytes" by K. G. Knauss, T. J. Wolery, and K. J. Jackson: Geochimica et Cosmocbimica Acta, v. 55, p. 1175-1176.

Millero, F. J., 1977, The use of the specific interaction model to estimate the partial molal volumes of electrolytes in seawater. Geochimica ct Cosmochimica Acta, v. 41, p. 215-223.

Millero, F. J., and Byme, R. H., 1984, Use of Pitzer's equations to determine the media effect on the formation of lead chloro complexes: Geochimica et Cosmochimica Acta, v. 48, p. 1145-1:50.

Monain, C., 1989, An ion interaction model for the volumetric propenies of natural waters: Density of the solution and partial molal volumes of electrolytes to high concentrations at $25^{\circ} \mathrm{C}$ : Geochimica et Cosmochimica Acta, r. 53, p. $1177-1188$.

Monain, C., and Schott, J., 1984, Determination of the solubility products of sodium carbonate minerals and an application to trona deposition in Lake Magadi (Kenya): Geocbimica et Cosmochimica Acta, v. 48, p. 571-581.

Morris, J. C., and Sturnm, W., 1967, Redox equilibria and measurements of potentials in the aquatic environment, in Gould, R. F., editor, Equilibrium Concepts in Natural Water Systems, Advances in Chemistry Series, v. 67, Anerican Cbemical Socicty, Washington, D. C., p. 270-285.

Nordstrom, D. K., and Jenne, E. A., 1977. Fluorite solubility equilibria in selected geothermal waters: Geochinica et Cosmocbimica Acta, v. 41. p. 175-188.

Nordstrom, D. K., Jenne, E. A., and Ball, J. W., 1979, Redox equilibria of iron in acid mine waters, in Jenne, E. A., editor, Chemical Modeling in Aqueous Systems, American Chemical Society Symposium Series, v. 93, s.merican Chemical Socicty, Washington, D. C., p. 51-79.

Nordstrom, D. K. and Munoz, J. L., 1985, Geochenical Thermodynamics: Benjamin/Cummings Publishing Company, Inc., Menlo Park, Califomia, 477 p.

Nordstrom, D. K., Plummer, L. N., Wigley, T. M. L., Wolery, T. J., Ball, J, W., Jenne, E. A., Bassett, R. L., Crerar, D. A., Florence, T. M., Fritz, B., Hoffman, M., Holdren, G. R., Jr., Lafon, G. M., Mattigod, S. V., McDuff, R. E., Morel, F., Reddy, M. M., Sposito. G., and Thralkill, J., 1979, A comparison of compurerized chemical models for cquilibrium calculations in anucous systems, in Jenne, E. A., editor, Chemical Modeling in Aqueous Systems, Annerican Chemical Society Symposium Series, v. 93, American Chemical Society, Washington, D.C. p. 857-892.

Pabalan, R. T., and Pitzer, K. S., 1987. Thermodyyamics of concentrated electrolyte mixtures and the prediction of mineral solubilitics to high temperatures for mixtures in the system Na-K-Ca-Mg-Cl-SO $4-\mathrm{OH}-\mathrm{H}_{2} \mathrm{O}$ : Geochimica et Cosmochimica Acta, v. 51, p. 2429-2443.

Pabalan, R. T., and Pitzer, K. S., 1988. Heal capacity and other themodynamic properties of $\mathrm{Na}_{2} \mathrm{SO}_{4(\text { aq }}$ in hydrothermal solutions and the solubilities of sodium sulfate minerals in the system Na-Cl-SO $4-\mathrm{OH}_{4}-\mathrm{H}_{2} \mathrm{O}$ : Geochimica ct Cosmochimica Acta, v. 52, p. 2393-2904.

Pabalan, R. T., and Pitzer, K. S., 1990, Mode's iur alyueous electrolyte mixiures for systems extending from dilute solutions to fused salts, in Mclchior, D. C., and Basselt, R. L., editors, Chemical Modeling of Aqueous Systems 
Il, American Chemical Society Symposium Serics, v. 416, American Chemical Society, Washington, D. C., p. 4457.

Parkhurst, D. L., Thorstenson, D. C., and Plurnmer, L. N., 1980, PHREEQE-A Computer Program for Geochemical Calculations: United States Scological Survey Water Resources Investigations 80-96.

Pitzer, K. S., 1973, Thermodynamics of electrolytes - I. Theoretical basis and general equations: Journal of Physical Chemistry, v. 77, p. 268-277.

Pitzer, K. S., 1975, Thermodynamics of electrolyies. V. Effects of higher-order electrostatic terms: Joumal of Solution Chemistry, v. 4, p. 249-265.

Pitzer, K. S., 1979, Theory: ion interaction approach, in Pytkowicz, R. M., editor, Activity Cocfficients in Electrolyte Solutions, CRC Press, Boca Raton, Florida, p. 157-208.

Pitzer, K. S., 1984, A consideration of Pitzer's equations for activity and osmotic coeflicients in mixed electrolytes: Joumal of the Chemical Society, Faraday Transactions 1, v. 80, p. 3451-3454.

Pitzer, K. S., 1987. Thermodynamic model for aqueous solutions of liquid-like densily, in Carmichael, I. S. E., and Eugster, H.P., cditors, Themodynamic Modeling of Geological Matcrials: Mincrals, Fluids and Melts, Reviews in Mineralogy, v. 17, Mineralogical Socicty of America, Washington, D. C., p. 97-142

Pitzer, K. S., 1992, Ion interaction approach: Theory and Data Comelation, in Pitzer, K. S., edilor, Activity Coefficients in Electrolyte Solutions, 2nd edition, CRC Press, Boca Raton, Florida, p. 75-153.

Pitzer, K. S., and Kim, J. J., 1974, Thermodynanics of clectrolytcs. IV. Activity and osmotic coefficients for mixed electrolytes: Joumal of the American Chemical Society, v. 96, p. 5701-5707.

Pitzer, K. S., and Mayorga, G., 1974, Themodynamics of electrolytes. III. Activity and osmotic coefficients for 22 electrolytes: Joumal of Silution Chemistry, v. 3, p. 539-546.

Pitzer, K. S., Roy, R. N., and Silvester, L. F., 1977, Thermodynamics of electrolytes. 7. Sulfuric acid: Journal of the American Chemical Society, v. 99, p. 4930-4936.

Pitzer, K. S., and Silvester, L. F., 1976, Thennolynamics of electrolytes. VI. Weak electrolytes including $\mathrm{H}_{3} \mathrm{PO}_{4}$ : Joumal of Solution Chemistry, v. 5, p. 269-278.

Plummer, L. N., and Parkhurst, D. L., 1990, Application of the Pitzer equations to the PHREEQE geochemical model, in Melchior, D. C., and Bassett, R. L., editors, Chemical Modeling of Aqueous Systems II, American Chemical Society Symposium Series, v. 416, American Chemical Society, Washington, D. C., p. 128-137.

Plummer, L. N., Parkhurst, D. L., Fleming, G. W., and Dunkle, S. A., 1988, A Computer Program Incorporating Pitzer's Equations for Calculation of Geochemical Reactions in Brines: United States Geological Survey WaterResources Investigations Report 88-4153, 310 p.

Prigogine, l., and Defay, R., 1954, Chemical Thermodynamics: Longmans, Green, and Company, London.

Reardon, E. J., and Langmuir, D., 1976, Activity coetficients of $\mathrm{MgSO}_{4}{ }^{\circ}$ and $\mathrm{CaSO}_{4}{ }^{\circ}$ ion pairs as a function of ionic strength: Geochimica et Cosnochimica Acta, v. 40, p). 549-554.

Saxena, S. R., 1973, Themnodyulunics of Rock-Foming Crystalline Solutions: Springer-Verlag, New York. 
Shock, E. L., and Helgeson, H. C., 1988. Calculation of the thermodynanic and transport properties of aqueous species at high pressures and temperatures. Correlation algorithms for ionic species and equation of state predictions to $S \mathrm{~kb}$ and $1000^{\circ} \mathrm{C}$ : Geochimica et Cosmochimica Acta, v. 52, p. 2009-2036.

Strock, E. L., and Helgeson, H. C., 1989, Corrections to Shock and Helgeson (1988) Geachimica et Cosmochimico Acta 52, 2009-2036: Geochimica et Cosmochimica Acta, v. S3, p. 215.

Sbock, E. L., and Helgeson, H. C., 1990, Calculation of the thermodynamic and transport properties of aqueous species at high pressures and temperatures: Standard partial molal properties of organic species: Geochimica et Cosmochimica Acta, y. 54, p. 915-945.

Shock, E. L., Helgeson, H. C., and Sverjensky, D. A., 1989, Calculation of the thermodynamic and transport properties of aqueous species at high pressures and temperatures: Standard partial molal properties of inorganic species: Geochimica et Cosmochimica Acta, v. 53, p. 2157-2183.

Shock, E. L., Oelkers, E. H., Johnson, J. W., Sverjensky, D. A., and Helgeson, H. C., 1992, Calculation of the thermodynamic properties of aqueous species at high pressures and temperatures: Effective electrostatic radii, dissociation constants and standard partial molal propertics to $1000^{\circ} \mathrm{C}$ and $5 \mathrm{kbar}$. Jourual of the Chemical Society Faraday Transactions, v. 88, p. 803-826.

Silling, S. A., 1983, Final Techuical Position on Documentation of Computer Codes for High-Level Waste Management: NUREG-0856, United States Nuclear Regulatory Comnission, Washington, D. C.

Silvester, L. F., and Pitzer, K. S., 1977, Thermodynamics of electrolytes. 8. Enthalpy and the effect of temperature on the activity coefficients, Joumal of Solution Chemistry, v. 7, p. 327-337.

Silvester, L. F., and Pitzer, K. S., 1978, Thermodynamics of elcetrolytes. X. High-temperature properties, including enthalpy an heat capacity, with application to sodium cbloridc: Joumal of Physical Chemistry, v. 7, p. 327-337.

Simonson, J. M., Roy, R. N., Roy L. N., and Johnson, D. A., 1987, The thermodynamics of aqueous borate solutions I. Mixtures of boric acid with sodium or potassium borate and chloride: Joumal of Solution Chemistry, v. 16, p. 791-803.

Spencer, R. J., Moller, N., and Weare, J. H., 1990, The prediction of mineral solubilities in natural wates: A chemical equilibrium model for the $\mathrm{Na}-\mathrm{K}-\mathrm{Ca}-\mathrm{Mg}-\mathrm{Cl}-\mathrm{SO}_{4}-\mathrm{H}_{2} \mathrm{O}$ system at temperatures below $25^{\circ} \mathrm{C}$ : Geochimica et Cosmochimica Acla. v. 54, p. 575-590.

Standard Methods for the Examination of Water and Wastewater, 14th edition, 1976, prepared and published jointly by the American Public Health Association, American Water Works Association, and Water Pollution Control Federation: American Public Health Association, Washington, D. C.

Stumun, W., and Morgan, J. J., 1981, Aquatic Chemistry, second edition: John Wiley and Sons, New York

Tanger, J. C., and Helgeson, H. C., 1988, Calculation of the thermodynamic and transport properties of aqueous species at bigh pressures and temperatures: Revised equations of state for the standard partial molal properties of ions and electrolytes: American Journal of Science, v. 288, p. 19-98.

Tardy, Y., and Garrels, R. M., 1974, A method of estimating the Gibbs energies of tomation of layer silicates. Geocbimica et Cosmochimica Acta, v. 38, p. 1101-1116.

Thorstenson, D. C.. 1970, Equilibriun distribution of small organic malecules in natural waters; Geochinica ct Cosmochimica Acta, v. 34, p. 745-770. 
Thorstenson, D. C., 1983, The concept of electron activity and its relation to redox potentials in aqueous geochemical systems: United States Geological Survey Open-Filc Report 84-072 (available from Opcn-Filc Services Section, Western Distribution Brancb, Box 25425, Federal Center, Denver, CO 80225).

Truesdell, A. H., 1968, The advantage of using pe rather than Eh in redox equilibrium calculations: Joumal of Geological Education, v. 16, p. 17-20.

Yan Zeggeren, F, and Storey, S. H., 1970, The Computation of Chemical Equilibrium: Cambridge University Press.

Viani, B. E., and Bruton, C. J., 1992, Modeling Fluid-Rock Intcraction at Yucca Mountdin, Nevada: A Progress Repor: UCRL-ID-I0992I, Lawrence Livemon: National Laboratory, Livemone, Califomia.

Willey, L. M., Kharaka, Y. K., Presser, T. S., Rapp, J. B., and Banes, I., 1975, Shon claain aliphatic acid anions in oil field waters and their contribution to the measured alkalinity: Geochimica ct Cosmochimica Acta, v. 39, p. 1707-1711.

Wolery, T. J., 1978, Sone Chemical Aspects of Hydrothermal Processes at Mid-Ocean Ridges-A Tbeoretical Study: Ph.D. thesis, Northwestem Universily, Evanston, Illinois.

Wolery, T. J., 1979, Calculation of Chemical Equilibrium between Aqucous Solutions and Miterals: The EQ3/6 Software Package: UCRL-52658, Lawrence Livemore National Laboratory, Livermore, Califomia.

Wolery, T. J., 1983, EQ3NR, A Computer Program for Gcocliemical Aqueous Speciation-Solubility Calculations. User's Guide and Documentation: UCRL-53414, Lawrence Livemore National Laboratory, Livermore, Califomia.

Wolery. T. J., 1990, On the thermodynamic framework of solutions (with special reference to aqueous electrolyte solutions: American Joumal of Science, v. 290, p. 296-320.

Wolery, T. J., 1992, EQ3/6, A Software Package for Geochemical Modeling of Aqucous Systems: Package Overview and Installation Guide (Version 7.0): UCRL-MA-1 10662-PT-1, Laurence Livermore National Laboratory, Livermore, Califomia.

Wolery, T. J., and Daveler, S. A., 1992, EQ6, A Computer Code for Rcaction-Path Modcling of Aqueous Geochemical Systems (Version 7.0): Theoretical Manual, User's Guide, and Related Documettation: UCRL-MA110662-PT-IV, Lawrence Livennore National Laboratory, Lisemore, Califomia.

Wolery, T. J., Jackson, K. J., Bourcier, W. L., Bruton, C. J., Viani, B. E., Knauss, K. G., and Delany, J. M., 1990, Current status of the EQ3/6 software package for geochemical modeling, in Melchior, D. C., and Basset, R. L., editors, Chemical Modeling of Aqueous Systems II, Americau Chenical Society Symposium Series, v. 416, Ankrican Cbemical Socicty, Washington, D. C., p. 104-116.

Wolery, T. J., and Walters, L. J., Jr., 1975, Calculation of equilibrium distributions of chemical species in aqueous solutions by means of monotone scquences: Mathematical Geology, y. 7, p. 99-115.

Wood, B. J., and Fraser, D. G., 1977, Elementary Thermodynamics for Geologists: Oxford University Press, Oxford, United Kingdom. 


\section{Appendix A: Glossary of Major Variables in EQ3NR}

This glossary covers the major variables in EQ3NR. Almost all of these are also shared by EQ6 (Wolery and Daveler, 1992). Those which are unique to EQ3NR are so marked. This glossary does not include variables which are of a purely local nature, such as a foating point variable used to accumulate sums in a do loop. Logarithmic quantities are frequently used. All refer to base ten, unless otherwise specified.

The variable names may be preceded by the corresponding algebraic symbols used in this report, if any. Following the conventions used universally in the EQ3/6 package, variables beginning with $\mathbf{i}, \mathbf{j}, \mathbf{k}$ or $\mathbf{n}$ are integer, those beginning with $\mathbf{q}$ are logical, and those beginning with $\mathbf{u}$ are character variables. Variables beginning with any other letters, including $\mathbf{l}$ and $\mathbf{m}$, should be real*8. On 32-bit machines, this corresponds to double precision; on 64-bit machines, this corresponds to single precision.

Sorre of the entries in this glossary are described as either "data file ordered" or "input file ordered". The former refers to the indexing of arrays that have been filled by reading the data file, data1. The latter refers to the indexing of arrays that have been filled by reading the input file. Since the two forms of indexing are not equivalent, EQ3NR must correlate them. This is done by mapping "input file ordered" data into a form which corresponds to "data file ordering," which is the ordering which is used in the code calculations. Most data arrays which are "input file ordered" are therefore treated as holding arrays and play no further role in the calculation once the data in them has been mapped to corresponding arrays which are "data file ordered."

Most arrays in EQ3/6 are dimensioned using FORTRAN parameters. The value of a parameter is assigned once in a code (in a PARAMETER statement) and can not be changed elsewhere.

Some FORTRAN compilers permit parameters to be passed through calling sequences, but others do not allow this, as a means of protecting the parameter's assigned value. A called module often requires the cimensions of passed arrays. This is handled in EQ3/6 by the use of "dimensioning variables," which are ordinary integer variables whose values have been set equal to the corresponding parameters. The names of the dimensioning parameters in EQ3/6 typically end in par, pa, or pa1. The names of the corresponding dimensioning variables end, respectively, in max, $\mathbf{m x}$, and $\mathbf{m x l}$. Thus, the dimension of the an matrix is given by the pair $\mathbf{k p a r} / \mathbf{k m a x}$. The maximum number of aqueous species is given by nstpar/nstmax. The parameter suffixes are used exclusively by dimensioning parameters. However, the corresponding variable suffixes are not used exclusively by dimensioning variables. For example, itermx is the maximum number of Newton-Raphson iterations.

$2 \quad 20$

aadh
The Jacobian matrix. Dimensioning: aa(kpar,kpar). Usage: aa(krow,kcol) is the element corresponding to the krow-th row and the kcol-th column. Note: In EQ3NR, this is also used as the matrix that is required for simultaneous solutions in pre-Newtol1-Raphson optimization.

Inteqolating polynonial coefficients for conı̧uting the Dehye-Hũckel paranketer $A_{\text {Y. } O}$ (adh) as a function of temperature. Dincensioning: andh(narxpa,ntprpa). Usage: aadh(narx,ntpr) refers to the narx-th coefficient for the ntpr-th temperature range. 
aaphi Interpolating polynomial coeficients for computing the Debye-Hückel parameter $A_{Q}$ (aphi) as a function of temperaturc. Dimensioning: asphi(narxpa,ntprpa). Usage: anphi(narx,ntpr) refers to the garx-th coefficient for the ntpr-th ternperature range.

abdh Interpolating polynomial coefficients for computing the Debye-Hückel parameter $B_{\gamma}$ (bdh) as a function of temperature. Dimensioning: aldh(narxpa,ntprpa). Usage: abdh(marx,ntpr) refers to the narx-th coefficient for the ntpr-th temperature range.

abdot Interpolating polynomial coefficients for computing the extended Debye-Hückel parameter $\dot{B}$ (abdot) as a function of tempcrature. Dimensioning: abdot(narxpa, ntprpa). Usage: abdot(narx,ntpr) refers to the narx-th coefficient for the ntpr-th temperature range.

$a_{w}, a_{i} \quad$ act

$\log a_{m n} \log a_{l} \quad$ acug

A $\quad$ adh

aeh

afenst

$A, A$ alf

$A, A$.

$A, A$.

altx

$\underline{\alpha}$ afficx

ags

alpha

al10

The activity of an aqueous species. Dinensioning: act(nstpar). Usage: act(ns) rcfers to the activity of the ns-th aqueous species.

The log activity of an aqueous species. Dimensioning: actlg(nstpar). Usage: actlg(ns) refers to the log activity of the ns-th aqueous species.

Debye-Hückel $A_{\gamma}$ parameter for computing the base ten logarithm of the molal activity coefficient of an aqueous species.

Interpolating polynomial coefficients for computing the special cquilibrium constant $\log K_{E h}$ (xlkeh) as a function of temperature. Dimensioning: aeh(narxpa, niprpa). Usage: aeh(narx,ntpr) refers to the narx-lh coefficient for the ntpr-th temperature range.

The affinity constant; equal to $0.001(2,303) R T$.

Affinity of a pure mineral to precipitatc, kcal. Dimensioning: aff(nmtpar). Usage: aft(nm) refers to the affinity of the am-th pure nineral.

Affinity of a solid solution phase to precipitate, kcal. Dimensioning: afflex(nxtpar). Usage: afficx(bx) refers to the affinity of the ax-th solid solution.

Afrinity of an end-member component of a solid solution. Dimensioning: allx(iktpar,xtpar). Usage: affx(ik,nx) refers to the affinity of the ik-th end-member component of the $\mathrm{nx}$-th solid solution.

Interpolating polynomial coeficients for computing the array of equilibrium constants xlkg. Dimensioning: ags(narxpa,ntprpa, ngtpar). Usage: ags(narx,ntpr,ng) refers to the narx-th cocfticient for the ntpr-th temperature range for the ng-th gas specics.

Residual funcion rector. Dimensioning: alpha(kpar). Usage: alpha(kcol) refers to the kcol-th master iteration variable.

In 10; note- the value 2.303 is geserally an insuffeient approximation; this parameter should be calculated to the full machine precision to avoid problems with numcrical consisiency. 
Interpolating polynomial cocfficients for computing the array of equilibrium constants xlkm. Dimensioning: ama(narxps,ntprpa,nmitpar). Usage: amn(narx, ntpr,nm) refers to the aarx-th coefficient for the ntpr-th temperature range for the nm-th pure mineral.

$A_{\phi}$

aphi

apress

ard

$p_{k w}$

ars

atwt

$a_{i}$

$B_{\gamma}$

B

$B_{\text {func }}$

$\beta_{\max }$

$\dot{b}$

bdh bdot Extended Debye-Hūckel B-dat parameter.

bfac Array of $(\beta+1)^{\frac{1}{n}}$ factors used in pre-Newton-Raphson optimization. Dimension-

Debye-Hückel $A_{\phi}$ parameter.

Interpolating polynomial cocfficients for computing the pressure (press) as a function of temperattre. Dimensioning: apress(narxpa,ntprpa). Usage: apress(narx, ntpr) refers to the narx-th cocfficient for the ntpr-th temperature range.

Holding array used to read in the interpolating polynomial cocficients used to computc equilibrium constants of various kinds of reactions. Dimensioning:

ard(narxpa, ntprpa). Usage: ard(narx, atpr) is the narx-th coefncient for the ntprth temperature range of the current reaction.

Solid solution activity coefficient model parameters. Dimensioning: apx(iapxpa,nxtpar). Usage: apx(iapx,nx) refers to the iapx-th cocfficient for the nx-th solid solution.

Interpolating polynomial coefficients for computing the array of equilibrium constants xlks. Dimensioning: ars(narxpa,ntprpa,nstpar). Usage: ars(marx,ntpr,ns) refers to the narx-th coefficient for the nipr-th temperature range for the ns-th aqueous species.

Atomic weight of a chemical element. Dimensioning: atwt(nctpar). Usage: atwl(nc) refers to the nc-th chemical element.

azero Hard core djamcter of an aqueous species. Dimensioning: azero(nstpar). Usage: azero(ns) refers to the ns-th aqueous species.

bbig The mass balance jesidual (an element of the beta array) which has the most extreme positive value; if none is positive, this is assigned a value of zero. The name of the associated basis species is given in the variable ubbig.

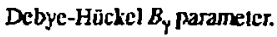

Residual function vector, with mass balance elements nomalized. Dimensioning: beta(kpar). Usagc: beta(kcol) refers to the $k$ col-th riaster iteration variable.

Convergence function that measures the improvement in betamx, defined as

\section{(J.0 - (betnmx/betmxo))}

betamx Max norm of B.

betmxo The previoas value of betamx. ing: bfac(kpar). Usage: brac(kcol) refers to the kcol-th master iteration variable. 
bgamx The max norm of activity coefficient residual functions; the name of the associated species is given in the variable ubgamx.

$\mu_{i j k}$ bmu Array of third order primitive virial coefficients of Pilzer's cquations $\left(25^{\circ} \mathrm{C}\right.$ values).
Dimensioning: bmu(nmupar). Usage: bmu(k) is the coefficient for the $k$-th species
triplet. See dmu1, dmu2, nmu, and pmu.

bnes The mass balance residual (an element of the beta array) which has the most extreme negative value; if none is negative, this is assigned a value of zero. The name of the associated basis species is given in the variable ulmeg.

$\begin{array}{ll}s_{\lambda}(n) & \text { bslm } \\ b & \text { bt }\end{array}$

$\mathrm{cco} 2$

$b_{s g} \quad$ cdre

$b_{s \phi} \quad$ cdrm

br edrs

$c_{\mathrm{Es}}$

cess

cessr
Array of second order "short range" prinutive virial coefficient parameters of Pitzer's cquations ( $25^{\circ} \mathrm{C}$ values). Dimensjoning; bslm(J,nslpar). Usagc: bsim(n,k) is the $n$-th such paraneter for the $k$-th species pair. Sec dslm1, dslm2, nslmx, pslm, and pslam.

The $b$ factor appearing in the Debye-Hinckel term in Pitzer's equations; it is assigned a constant value of 1.2 .

Parameters of the Drummond (1981) equation for computing $\log \gamma_{\mathrm{CO}_{2(a q)}}$ as a function of temperature and ionic strength. Dimensioning: $\operatorname{cco2}(5)$. Usage: $\operatorname{cco2}(i)$ refers to the $i-$ th coefficient.

Reaction cacficient for dissolution reactions of gas species. Dimensioning: cdrg(nsqpa1,ngtpar). Usage: cdrg(ns,ng) is the coefficient of the ns-th aqueous basis species appearing in the dissolution reaction for the ng-th gas; cdrg(nsq1,ng) is the coefficient of the ng-th gas itsclf.

Reaction coefficient for the dissolution reactions of pure minerals, Dimensioning: cdrm(nsqpa 1,nmtpar). Usage: cdrm(ns, am) is the coefficient of the ns-th aqueous basis species appearing in the dissolution reaction of the nm-th pure mineral; cdrm(nsq1,nm) is the coefficient of the nm-th pure mineral itself.

Reaction coefficient for aqueous redox and dissociation reactions. Dimensioning: cdrs(nsqpal,nrstpa). Usage: cdrs(ns,nrs) is the coefficient of the ns-th aqueous basis species appearing in the nrs-th aqueous dissociation/destruction reaction; edrg(nsql,nrs) is the coefficient of the associated species (of index nse = nrs + nsb). If the ns-th species is an auxiliary basis species and nrs is its own formally associated reaction, edrs(ns,nrs) $=0$ and cors(nsq1,nrs) is its coefficient.

cdrsd Holding array used to read in the coefficients of reactions listed on the data file. Dimensioning: cdrsd(nsqpal). Usage: cdrsd(n) is the n-th coeficient in the cursent reaction as it is written on the data file; it corresponds to the species whose name is udrxd(n).

Array containing the elenxental composition coefficients of aqueous species. Dinensioning: cess(nctpar,nstpar). Usagc: cess(nc,ns) is the coefficient of the ne-th chemical clement for the ns-th aqueous species.

Holding aray used to read in the elemental composition cocficients of species listed on the data file. Dimensioning: cessd(nstipal). Usage: cessd(n) is the $n$-th coef- 
ficient for the current species as it is written on the data file; it corresponds to the element whose name is uelemd(n).

cessi Temporary storage array used to decode the clemental composition coefficients of species listed on the data filc. Dimensioning: cessi(nsqpa1). Usage: cessi(nc) is the coefficient for the nc-th element. This array is used to transfer data between the cessd array and the cess array.

$m_{i} \quad$ con

$\log m_{l} \quad$ conclg

$m_{i} \quad$ csort

csp

cspb

$\operatorname{cstor}$

$u_{s}{ }^{\prime}$

$\operatorname{csls}$

cte

$\underline{\delta}$

$\delta_{\text {fune }} \quad$ delfinc

$\delta_{\max }$

delmax

delmxo
Molal concentration of an aqueous species. Dimensioning: conc(nstpar). Usage: conc(ns) is the molal concentration of the ns-th aqueous species. This is defined as zero for water, cven its molality is tecinnically fixed at $\Omega(\approx 55.51)$.

Log molal concentration of an aqueous species. Dinkensioning: concig(nstpar). Usage: conclg(ns) is the log molal concentration of the ns-th aqueous species.

Molal concentrations of the aqueous species, sorted in order of increasing valuc. Dimensioning: csort(nstpar). Usage: primarily as a storage array required for the sor1ing; $\operatorname{csort}(\mathbf{n})=\operatorname{conc}(j \operatorname{sor}(\mathbf{n}))$. It is unique to EQ3NR.

Constraining parancter array: the meaning of cach member of this array is determined by tle corresponding mernber of the jflag array. Dimensioning: esp(nsqpa1). Usage: csp(ns) is the constraining parameter for the ns-th aqueous basis species. This array is data filc ordered. It is unique to EQ3NR.

Holding array into which the esp values are read from the input file. Dimensioning: $\operatorname{cspb(nsqpa1).~Usage:~} \operatorname{cspb}$ (n) is the constraining parameter for the $n$-lb aqueous basis species listed on the input file. This array is input file ordered. It is unique to EQ3NR.

Stoichiometric mass balance factors of aqueous specics for oxygen in the $O^{2-}$ valence form and hydrogen in the $\mathrm{H}^{+}$valence form. Dimensioning: cstor(2,nstpar). Usage: cstor(1,ns) is the factor for the ns-th aqueous species in the mass balance for $O^{2} ;$ cstor $(2, \mathrm{~ns})$ is the corresponding factor in the mass balance for $\mathrm{H}^{+}$. This array is used to set up the corresponding parts of the csts array. It is unique to EQ3NR.

Mass balance coefficient array. Dimensioning: csts(nsqpa1,nsipar). Usage: ests(nse,ns) is the coefficient of the ns-th aqueous species in the mass balance equation for the nse-th basis species. It is unique to EQ3NR.

Equivalent total molality of a chemical element in a given valence form or combination of valence forms in mutual equilibrium. Dimensioning: cte(nsqpa1). Usage: cte(ns) is the equivalent total molality of the ns-th aqueous basis specics.

Newton-Raphson correction term vector. Dimensioning: del(kpar). Usage: del(keol) is the conection lerm for the kcol-th master itcration yariable.

Convergence function that meastires the improvensent in delmax, defined as (1,0 - (delmax/delmxo)).

Max nom of $\underline{\not}$.

The previous value of delmax. 
$\frac{d \mu_{i j k}}{d T}$

$\frac{d^{2} \mu_{i j k}}{d T^{2}}$

$\frac{d^{S} \lambda_{i j}^{(n)}}{d T}$

$\frac{d^{2} s_{\lambda}^{(n)}}{d T^{2}}$

$E h$

$E_{\lambda(1)}, E_{\lambda^{\prime}(1)} \quad$ elam

F

$f_{O_{2}}$ $d \operatorname{dog} x w$

dmul

dmu2

dslm1

dsim2

ee

efac

eh

eps100

farad

fo2
Array of partial derivatives of the log mole fraction of water with respect to the log molalitics of the solute basis species. Dimensioning: dlogxw(nsqpar). Usage: dlogxu(ns) is the partial derivative with respect to the ns-th basis species.

Amay of first derivatives with respect to temperature of third order primitive virial coefficients of Pitzer's equations ( $25^{\circ} \mathrm{C}$ values). Dimensioning: dmu1(nmupar). Usage: dmu l(k) is the derivalive for the $k$-th species triplet. Sce bmu, dmuz, nmux, and pmu.

Array of second derivatives with resqeet to tenukerature of third order primitive virjal coefficients of Pitzer's equations ( $25^{\circ} \mathrm{C}$ values). Dinensioning: dmuz(nmupar). Usage: dmu l(k) is the derivative for the k-th species triplet. Sec bmu, dmul, nmux, and pmu.

Array ol first derivatives witl respect to temperature of second order "short range" primitive virial coefficient parameters of Pitzer's equations $\left(25^{\circ} \mathrm{C}\right.$ values). Dimensioning: dslm1(3,nslpar). Usage: dslm $1(n, k)$ is the $n$-th such paranicter for the $k$ th species pair. Sec bslm, dsim2, nslmx, psinu, and pslam.

Array of second derivatives with respect to temperature of second order "short

range" primitive virial coefficiem parauneters of Pitzer's equations $\left(25^{\circ} \mathrm{C}\right.$ values). Dimensioning: dsIm2 23 , nslpar). Usage: dslm2(n,k) is the n-th such parameter for the k-th species pair. See bslm, dslm1, nslmx, pslm, and pslam.

A work array used in solving matrix eqtations. Dimensioning: ee(kpar).

Array of recifrocal stoichiometric weighting factors used in pre-Newton-Raphson optimization; this is the $\frac{1}{n}$ which appears in the array of $(\beta+1)^{\frac{1}{n}}$ factors used in this optimization method. Dimensioning: efac(nsqpal). Usage: efac(ns) is the factor for the ns-th basis species.

Redox potential, volts.

The higher order electrostatic lambda functions that appear in Pitzer's equations. Di-

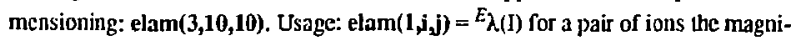
tude of whose clarges are $\mathbf{i}$ and $\mathbf{j}$; $\operatorname{clam}(2, \mathbf{i}, \mathbf{j})=E_{\lambda}$ (I) for the same pair of ions;

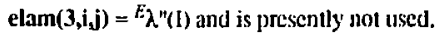

Onc hundred times the real $* 8$ machine epsilon.

The Faraday constam, 23062.3 cal/culuivalent-roll.

Oxygen fugacity, bars. 

$\log f_{O_{2}}$
forlg
Log oxygen fugacity.
$f_{8}$
fug
$\log f_{8} \quad$ fuglg
$g\left(\alpha_{n} \sqrt{\jmath}\right)$
$\boldsymbol{b}$
$g^{\prime}\left(\alpha_{n} \sqrt{l}\right)$
gp
$\log \lambda_{n} \log \gamma_{i} \quad$ glg
glgo
gm
iapxmx
iapxpa
ibasis
ibetmx
ibswx
$s_{z}$
Array of fugacities of gas species. Dimensioning: fug(ngtpar). Usage: fug(ng) is the fugacily of the ng-th gas species.
Array of $\log$ fugacitics of gas species. Dimensioning: fuglg(ngtpar). Usage: fuglg(ng) is the log fugacity of the ng-th gas species.
The funclion $g(x)$ which appears in Pitzer's equations. Dimensioning: $g(2)$. Usage: $g(1)=g\left(\alpha_{1} \sqrt{I)}\right.$ and $g(2)=g\left(\alpha_{2} \sqrt{I}\right)$.
The function $g^{\prime}(x)$ which appears in Pitzer's equations. Dimensioning: $g p(2)$. Usage: $E P(1)=g^{\prime}\left(\alpha_{1} \sqrt{I}\right)$ and $g p(2)=g^{\prime}\left(\alpha_{2} \sqrt{l}\right)$.
Amay of log activity coefficients of aqueous species; this is a mole fraction activity coefficient for water and a molal activity coefficient for all solute species. Dimen- sioning: $g \lg$ (nstpar). Usage: $g \operatorname{gg}(n s)$ is the log activity coefficient of the ns-th aque- ous species.
Array of previous values of $\log$ aclivity cocfficients of aqueons species Dimension- ing. glgo\{nstpar).
A work array that is used to store a copy of aa, the Jacobian matrix. Dimensioning: gm(kpar,kpar).
Maximum number of cocfficients for determining activity coefficient parameters per solid solution in the apx array. This is the variable equivalent to the parameter iapxpa.
Dimensioning parameter. the maximum number of coefficients for detennining ac- tivity cocficient parameters per solid solution in the apx array. Sec iapxmx.
Array that contains a record of basis switching. Dimensioning: ibasis(nstpar). Us- agc: if ibasis(ns) is not 0 , then the ns-th species was switched with the species whose index is given by ibasis(ns).
The index of the clement of the beta array corresponding to the max norm (betamx).
Array that contains the indices of species which are candidates for switching into the basis set. This is a part of the automatic basis switching mechanism. Dimensioning: ibswx(nsqpar). Usage: if ibswx(ns) is not 0 , then it gives the index of a species to switch with the ns-1h basis species.
iebal The index of the a(jucous species chosen for electrical balancing.
ielam Fag controlling whether or not higher order electrostatic terms are used in Pizzers erpations. It is sct by the uelam flag on the supporting data file and has the following meanings:
$=-1$ Higher order electrostatic tems are not used
$=1$ They are used 
An error flag parameter commonly found in subroutine calling sequences. Valucs greater than zero mark error conditions. Values less than zero mark warning conditions. The significance of this flag is only that the subroutine did or may have failed to carry out its primary function. For example, a matrix solver may have found a matrix to be computationally singular. This situation may or may not equate to an error in the context of the larger code. For example, the larger code may not have a problem with the fact that a matrix is computationally singular.

index Array containing the indices of tbe basis species for which the starting ilcration values must be estimated simultaneously. Dimensioning: iindex(nsqpar). Usage: index(n) is the $\mathbf{n}-$ th such species.

iindx 1 Array that contains the indices of the species for which the master iteration variables are deflocd. Dimensioning: findxl(kpar). Usage: indx 1 (kcol) is the index of the species corresponding to the kcol-th master iteration variable.

iktmax The maximum number of end members in a solid solution. This is the variable which corresponds to the dimensioning parameter jktpar.

iktpar Dimensioning parameter. the maximum number of cnd members in a solid solution. See iktmax.

insgf Flag array for determining how to compute the activity cocfficient of a neutral solutc species in conjunction with the B-dot equation. Dirnensioning: insgf(nstpar). Usage: insgf(ns) is the flag for the ns-th aqueous species:

$=0$ Set $\log \gamma_{i}=0$ (polar specics)

$=-1$ Use the Drumnond (1981) polynomial (non-polar species).

iodb1, etc. Debugging print option switches; sec Chapter 6. Some of these switches differ from those used in EQ6.

iopg 1, etc. Activity coefficient option switches; see Chapter 6.

iopr1, elc., Print oplion switches; see Chapter 6. Some of these switches differ from those used in EQ6.

iopt1, etc. Model option switches; see Chapter 6. Some of these swilches differ from those used in EQ6.

ir A work array used in solving matrix equations. Dimensioning: ir(kpar).

irang The real*8 inachinc exponent range paraneter (the exponent range is \pm irang).

istack A work array used to sort aqueous specics in increasiıg order of concentration. Dimensioning: istack(nstpar).

itermx Linit on the number of Newton-Rajplsson iterations.

izm The max norm on the electrical charges of ule agucous specics.

jcflag Status tlag array for clentical clencents. Dimensioning: jctlag(nctpar). Usagc: jeflag(nc) is the flag for the nc-th element:

$=0$ An clement does not appear in the current inodel

$=1$ It does appcar 
jflag Switch array that defines the type of constraint (governing equation) applied to each basis species. Dimensioning: jflag(nsqpar). Usage: jfag(ns) is the flag for the nsth basis species. See Chapter 6 .

jflagb Array used to read in jifag values from the input file. Dimensioning: jflagh(nsqpar). Usage: jnagb(n) is the jtlag value for the $n$-th basis species read from the input filc. This array is thus input file ordered. It is unique to EQ3NR.

jeflag Status switch array for gas species. Dimensioning: Jgflag(ngtpar). Usage: jeflag(ng) is the flag for the ag-th gas:

$=\mathbf{0}$ The gas species appears in the current model

$=2$ It is thermodynamically suppressed

jkflag Status switch array for solid solution end-member components. Dimensioning; jkflag(iktpar,pxtpar). Usagc: jkflag(ik,nx) is the flag for the ik-th end-member component of the nx-th solid solution:

= 0 The component appears in the current model

$=2 \mathrm{It}$ is thermodynamically suppressed

$=4$ It could not be found among the pure minerals

jmflag Status switch array for pure mincrals. Dimensioning: jmflag(nmtpar). Usage: jmflag(nm) is the flag for the nn-th pure mincral:

$=0$ The mineral appears in the current model

$=2 \mathrm{I} I$ is themmodynamically suppressed

jrs The indices of the aqueous reactions, sorted in increasing order of concentration of the associated species. Dimcosioning: jrs(nrstpa). Usage: jrs(n) is the index of tie reaction which corresponds to the non-basis aqueots species whose concentration is n-1h in this order. This array is used in nuaking sorted summalions.

jsfiag Status switch array for aqueous species. Dimensioning: jsflag(nstpar). Usage: jsflag(ns) is the flag for the ns-th aqueous species:

$=0$ The species appears in the current model

$=2$ It is themodynamically suppressed

$=3$ It does not appear in the curment model

jsol Array whose valucs define chosen models of activity coefficicnts in solid solutions. Dimensioning: jsol(nx/par). Usage: jsol(nx) is the flag for the $n x-$ th solid solution: $=0$ Ideal solution (Tbe data files currently support no other options.)

jsort The indices of the agueous species, sorted in increasing order of concentration. Dimensioning: jsort(nstpar). Usage: jsort( $n$ ) is the index of the aqueous species whosc concentration is $\mathbf{n}-\mathbf{t b}$ in this order. This array is used in making sorted summations.

jstack A work array uscd in sorting agueous species in increasing order of concentration. Dimensioning: jstuck(nstpar).

jxflag Status switch array for solid solutions. Dimensioning: jxfag(nxtpar). Usagc:

jxflag(nx) is the flag tor the nx-th solid solution;

$=0$ The phase appears in the current noolel

$=4$ It has no aclive cnd-member components 
jxmod Flag specifying the type of a species/reaction affected by an alter/suppress option specified on the input filc. Dimcnsioning: jxmod(nxmdpa). Usage: jxmod(n) is the flag for the n-th such option specified (see kxmod, uxmod, xlkmod, and nxmod):

$=0$ Agucous species/reaction

$=1$ Pure nuneral

$=2$ Gas

$=3$ Solid solution

ket Number of chemical elements present in the aqueous system curnently being modcled.

ketion A pointer array used in pre-Newton-Raplson optinization. It points to entrics in the subset of master iteration variables which nust be solved sinuliancously which correspond to basis species used to complete the delinition of input constraints for other basis species. Dimknsioning: kction(nsqpar). Usagc: kction(n) is the index in this subset for the basis species whiclu completes the constraint definition for the basis species whose index in this subset is $n$. It is unique to EQ3NR.

kdim Dimension of the Jacobian nutrix; $k$ dim $=\mathbf{k s q}$ in EQ3NR.

kebal Variable denoting the position of uhe species selected for clectrical balancing in the set of master iteration variables. It is tuigue to EQ3NR.

khydr Variable denoting the position of $\mathrm{H}^{+}$in the set of master iteration variables.

kkndex Array which narks those basis species whose concentrations must be initially estimated simultancously. Dimensioning:

kkndex(nsqpar). Usage: kkndex(ns) is the flag for the ns-th such species:

$=0$ Do not estimate simulianepusly

$=1$ Estimatc sinultancously

It is unique to EQ3NR.

kmax The maxinum number of master varialbles redable by EQ6. This is the variable corresponding to the jaranketer kpar.

kpar Dimensioning parameter: the miximum number of master variables readable by EQ6. See kmax.

ksb Variable denoting the position of the species used as the redox variable, currently $O_{2(g)}$ only; $\mathbf{k s b}=\mathbf{k c t}+1$.

ksq The nunber of active basis speics.

kxmod Flag defining the tyje of alter/suppress option specified on the input file. Dimensioning: kxmod(nxmdpa). Usage: $\operatorname{kxmod}(n)$ is the flag for the $n$-th such option specified (see j.mod, uxmod, xlkmod, and nxmod):

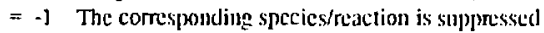

- 0 Its log $K$ is replaced by xlkmod

$=1$ Its $\log K$ is augnented by xlkmod

$=2$ Samc as $=1$, bu xlknum is given in '!njts of kcal/mole

$\log \lambda_{\text {ow }} \quad$ lamig Array of $\log$ activity cocficients of solid solution cul-member components. Dimensioning: lamlg(iktpar,nxtpar). Usage: lamglg(ik,nx) is the activity coefficicnt for the ik-th eud-member componcut ol the nx-th solid solution. 

$m_{T, \varepsilon} \quad$ mte
$M_{i} \quad$ mwiss
nadI
nalpha A pointer array used to find the $\alpha_{1}$ and $\alpha_{2}$ parameters of Pizer's equations for a spe- cies pair for which $S_{\lambda_{l}}$ and $S_{\lambda^{\prime}}$ values are to lec calculated. Dimensioning: ualpha(uslpar). Usage: palpha(1,nalpha(n)) $=\alpha_{l}$ and palpha $\left(2\right.$, alpha(n)) $=\alpha_{2}$ for the n-th such solute species pair.
nappar
napt
Array of total molalities of dissolved chemical elements. Dimensioning: mte(nctpar). Usage: mte(nc) is the molality of the ne-th element.
Array of molecular weights of aqueous species. Dimensioning: mwtss(nstpar). Us- age: mwtss(ns) is the molecular weight of the ns-th aqueous species.
The unit number of the datal file.
Dimensioning parameter: the maximum number of distinct pairs of $\alpha_{1}, \alpha_{2}$ parame- ters of Pitzer's equations. Sce napt.
The maximum number of distinet pairs of $\alpha_{1}, \alpha_{2}$ parameters of Pizer's equations. This is the variable corresponding to the dimensioning parameter nappar.

barxmx

$\sigma_{T, 4}$

$\varepsilon_{T}$

The maximum number of coefficients per temperature range for an interpolating polynomial coefficient array such as ars and amn. This is the variable comesponding to the parameter narxpa.

narxpa Dimeusioning parameter. the naximum number of coefficients per temperature range for an interpolating polynomial coefficient array. Sec narxmx.

nchlor The index of the species $\mathrm{Cr}$.

ncomp Array giving the number of end-member components in a solid solution. Dimensioniug: ncomp(nxtpar). Usage: ncomp(nx) is the number of such components for the $\mathrm{nx}$-th solid solution.

ncompb Array giving the number of solid solution end-member components of solid solutions for which nole fractions are read from the input fle. Dimensioning: ncompb(nxipar). Usage: ncompb(nxb) is the number of such components for the nxb-th solid solution so listed on the input file.

net Total number of chemical elements. netmax The nuximum number of chemical elements. This is a variable which comesponds to the parameter nctpar.

netpar Dimensioning paraneter. the maximum number of chemical elements. Sec nctmax.

nend Array that stores the indices of pure minerals that correspond to end-menber components of solid solutions. Dinensioning: nend(iktpar,nxtpar). Usage: nend(ik, nx) is the pure mineral index for the ik-th component of the ax-th solid solution.

nfac Artay of indices of doninant species. Dimensioniug: nfac(nsqpar). Usage: nfac(ns) is the index of the dominant species in the mass balance for the ns-th basis species. 
ngtmax The maximum number of gas species. This is a variable which corresponds to the parameter ngtpar.

ngtpar Dimensioning parameter: the maximum number of gas species. See ngtmax.

nhydr The index of the specics $H^{+}$.

ninpt The unit number of the input file.

ninpts The unit number of the file which is a copy of the input file that las been stripped of comment lines.

nmlx The number of entries in the nmxx pointer array, which is used in evaluating terms in the $\mu_{\text {ly }}$ cocfficients in Pilzer's cquations.

nmt

Total number of pure minerals.

nmtmax Maximum number of pure minerals.

nmtpar Dimensioning parameter: the maximum number of pure mincrals. See nmtmax.

nmupar Dimensioning parameter: the maximum number of species triplets with $\mu_{j ; f}$ cocflicients specified for use in evaluating Pitzer's equations. See nmul.

nmu The number of species triplets with $\mu_{i j k}$ coefficients specified for use in cvaluating Pitzer's equatious.

amut The maximum number of species triplets with $\mu_{i j k}$ coefficients specified for use in evaluating Pitzer's equations. This is the variable corresponding to the dimensioning parameter nmupar.

nmux Array identifying the specics in triplets with $\mu_{i j k}$ coefficients specilicd for use in evaluating Pitzer`s equations. Dimensioning: nmux(3,nmupar). Usage: nmux $(i, k)$ is the index of the $i-t h$ species in the $k$-th triplet.

nmxi A pointer array which gives the range in the nmux array. Dimensioning: nmxi(2,nstpar). Usage: nmxi( $1, n s)$ to $n m x i(2, n s)$ is the range in the $n m x x$ array corresponding to the species whose index is ns.

nmxpar Dimensioning parameter: the maximum number of entries in the nmxx pointer array, which is used in evaluating terms in the $\mu_{j j k}$ cocfficients in Pitzer's equations. To be absolutely safe, one could set nmxpar equal to 3*ninupar. Sec nmxt.

nmxx Alt ordered pointer array constneted from the nmux altay which, when used in conlkelion with the nmxi pointer array, gives for each solute species the indices of the other two specics appearing in a trij)let for a $\mu_{i j k}$ coefficient in Pitzer's equations and also the index of the triplet itsell. Dinnensioning: nmxx(3,nmapar). Usage: nmxx $(1, \mathbf{k k})$ is the index of the first spccies it the kk-th cutry, nmox $(2, k k)$ is the intdex of the second species in this entry, and $\operatorname{nmx}(3, \mathbf{k k})$ is the index of triplet itself. For a given species whose index is ns, the corresponding range in the nmxx artay is defined by $k \mathrm{k}$ in the range from nnxi(1,ns) to nmxi(2,ns). This combination of 
pointer anrays is useful in evaluating the sum, $3 \sum_{j} \sum_{k} \mu_{i j k} m_{j} m_{k}$, which appears in the expression for the solutc activity coefficient.

nmxt The maximum number of entries in the nmxx pointer array, which is used in evaluating terms in the $\mu_{i j k}$ coefficients in Pitzer's equations. This is the variable corresponding to the dimensioning parameter nmxpar.

noutpl The unit number of the output filc.

npkup The unit nun:ber of tue pickup fle.

$r_{T}$

$s_{B}$

nsim!

nsimx

nsk

nsp
Total number of reactiors among aqueous species.

nrstmx The maximum number cf aqueous reactions. This is a variable corresponding to the parameter nrsipa.

nrstpa Dimensioning paramct : the maximum number of aqueous reactions. See arsinx.

nsb The number of strict basis species. Also the index denoting the aqueous redox species (currently $\mathrm{O}_{2}$ ); nsb $=$ nct +1 .

nsbl Index denoting the first species in the auxiliary basis set; nsb1 $=\mathbf{n s b}+1$.

nslpar Dimensioning parametcr: the maximum number of species pairs having $s_{\lambda}^{(n)}$ parameters specified for er 1 luation of Pitzer's equations. Sec nslmt.

The number of species [ airs with $s_{\lambda_{i j}(n)}$ parameters speciffed for use in cvaluating Pitzer's cquations.

The naxinum number of sjecies pairs with $s_{\lambda}^{(n)}$ parameters specified for evaluation of Pitzer's equations. This is the variable correspoding to the dimensioning parametcr nslpar.

Array identifying the species in pairs with $s_{\lambda}(n)$ parameters specified for use in evaluating Pitzer's equatiuns. Dimensioning: nsimx(2,nslpar). Usage: $n s \operatorname{ma}(i, k)$ is the index of the $i$-th species in the $k-t h$ pair.

The number of entries $i_{i}$ the nsxx pointer anray, which is used in cvaluating terms in the $s_{\lambda}(n)$ parameters in Pitzer's equations.

Array contaituing the incices of the aqueous, mineral, solid solution end-member component, or gas species that are regutired to define certain kinds of constraints on basis species on the inp: file. Dimensioning: nsp(nsqpar). Usagc: nsp(ns) is the index of the species for the constraint place on the ns-th basis specics. This never refers to the ns-th basis pecies itself, but always a second species. Phase equilibrium (solubility) constrai ts and log activity combination and mean log activity constraints all require the s].ecification of such second spocics. This array is data file ordeced. It is tuingut to EQ3NR. 
nspec

$s_{e}$

nsq

nsq1

nsqb

nsqmax

nsqmx1

nsqpa1

nsqpar

$s_{T}$

nst

ustmax

nstpar

n5xi

nsxpar

ns.xt

ns.xx
Array that contains the indices of the basis species which appear on the input file. Dinensioning: nspec(nsquar). Usage: nspec(n) is the index of the $\mathbf{n}$-th basis species appearing on the input file. It is thus input file ordered. It is unique to EQ3NR.

Number of aqueous basis species.

The yariable equivalent $10 \mathrm{nsq}+1$. This is used in reaction coefficient arrays to mark the coefficient of the species formally associated with a given reaction. For example, cdrs(nsq1,nrs) refers to the coefficient of the associated aqueous species destroyed in the nrs-th reaction.

Number of basis species which appear on the input file.

The maximum number of aqueous basis species. This is the variable corresponding to the parameter nsqpar.

A variable equal to nsqmax +1 . This corresponds to the paraneter nsqpat.

Dimensioning parameter: the equivalent of nsqpar +1 . See nsqmx1.

Dimensioning parameter: the maximum number of aqueous basis species. See nsqmax.

Total number of aqueous species; this currenty includes all basis species but only those non-basis species appearing in the system for a given problem defined on the input file.

The maximum number of aqueous species. This is a variable conesjonding to the parameter nstpar.

Dimensioning parameter. the maximum number of aqueous species. See nstmax.

A pointer array which gives the range in the nsxx array. Dimensioning: nsxi(2,nstpar). Usage: nsxi(1,ns) to nsxi $(2, n s)$ is the range in the nsxx array corresponding to the species whose index is ns.

Dimensioning parameter: the maximum number of entries in the nsxi pointer array, which is used in evaluating tems in the $s_{\lambda_{i j}}$ coefficicnts in Pitzer's equations. To be absolutely safc, one could set nsxpar equal $102^{*}$ nmupar. See nsxt.

The maximum number of entries in the nsxi pointer array, which is used in evaluating terms in the $s_{\lambda_{i j}}$ coefficients in Pitzer's equations. This is the variable corresponding to the dimensioning parameter nsxpar.

An ordered pointer array constnucted from the nsimx array which, when used jll connection with the nsxi pointer array. gives for each solute species the index of the other species appearing in a pair for the $s_{\lambda_{i j}}$ cocfficients in Pitzer's equtations and also the index of the pair itsele. Dimensioning: nsxx(2,nsxpar). Usige: nsxx (1,kk) is the index of the other species in the kk-th cntry ant nsxx(2,kk) is the pair itself. For a given syecies whose index is ns, the corresjonding tange in the nsxx array is defined by $\mathbf{k k}$ in the range from nsxi( 1, ns) to nsxi $(2, n s)$. This conbinbtion of point- 
er arrays is useful in evaluating the sum, $2 \sum_{j} \lambda_{i j} m_{j}$, which appears in the expression for the solute activity coefficient.

ntill The number of lines of the tite on the input file.

ntild The number of lines of the tite on the datal file.

utitux The maximum number of lines of the tiule on the inpot or dalal file. This is a variable corresponding to the parameter ntitpa.

ntitpa Dimensioning parameter: the maximum number of lines of the titke on the input or datal filc. See atitmx.

nepr The index of the temperature range corresponding to the temperature specified on the input file.

atprrmx The maximum number of temperature ranges for an interpolating polynomial coetficient amay such as ars and amn. This is a variable corresponding to the parameter itprpa.

ntprpa Dimensioning parameter. the naximum number of temperature ranges for an interpolating polynomial co.fficient array such as ars and amn. See ntprmx.

ntiyo The unit number of the screen file.

uxumdmx The maximum number of alter/suppress options that cau be specified on the input file. This is a variable zorresponding to the parameter nxmdpa.

nxmdp2 Dimensioning parameter, the maximum number of alter/suppress options that can be specified on the input file. See nxmdmx.

nxmod The number of species/reactions affected by alter/suppress options specified on the input file. See jxmod, kxmod, uxmod, and xlkmod.

nxt Total number of solid solution phases.

nxtb Number of solid solutions whose compositions are read from the input filc. It is unique to EQ3NR.

extmax The maximum number of solid solutions. This is a variable corresponding to the parameter axtpar.

extpar Dimensioning parameter: the maximum number of solid solutions. See nxtmax.

$\Omega$

$\log \Omega$

$\phi$

$\alpha_{1}, \alpha_{2}$ om

omig

oscotr

palpha
The water constant, $1000+$ the molecular weight of water $(\Omega \approx 55.51)$.

Log water constant.

The osmotic coefficient.

The $\alpha_{1}$ and $\alpha_{2}$ parameters of Pitzct's equations. Dimensioning: palpha(2,nappar). Usage: palpha $(1, k)=\alpha_{1}$ and palpha $(2, k)=\alpha_{2}$ for the $k$-th distinct pair of $\alpha_{I}, \alpha_{2}$ 


\begin{tabular}{|c|c|c|}
\hline pe & pe & The eloctron activity function; $p e=-\log a_{e}$. \\
\hline$\mu_{i j k}$ & pmu & $\begin{array}{l}\text { Array of third order primitive virial coefficients of Pitzer's equations (values at the } \\
\text { current specified temperature). Dimensioning: pmu(nmupar). Usagc: pmu(k) is } \\
\text { the coefficicat for the k-th species triplet. Sce bmu, dmu1, dmu2, and nmux. }\end{array}$ \\
\hline $\boldsymbol{P}$ & press & Ptessure, bars. \\
\hline$s_{\lambda_{i j}}$ & pslam & $\begin{array}{l}\text { Array of second order "short range" primitive virial cocfficients of Pitzer's equa- } \\
\text { tions (values at the current specified temperature). Dimensioning: pslam(3,nslpar). } \\
\text { Usage: pslam(n,k) is the n-th such coefficient for the k-th species pair. Sce bslm, } \\
\text { dsim1, dsim2, nslmx, and psim. }\end{array}$ \\
\hline \multirow[t]{3}{*}{$s_{\lambda}(n)$} & psim & $\begin{array}{l}\text { Amay of second order "short range" primitive virial cocfficient parameters of } \\
\text { Pilzer's cquations (values at the cument specified temperature). Dimensioning: } \\
\text { pslm(3,nslpar). Usage: psim(n,k) is the n-th such parameter for the } k \text {-th species } \\
\text { pair. Sec bslm, dsim1, dslmz, nslmx, and pslam. }\end{array}$ \\
\hline & qbassw & Logical nag indicating whelher or not basis switching has taken place. \\
\hline & qbswx & $\begin{array}{l}\text { Logical flag indicating whetber or not there are candidates for automatic basis } \\
\text { switcining. }\end{array}$ \\
\hline \multirow[t]{2}{*}{$\boldsymbol{R}$} & rconst & The gas constant, $1.98726 \mathrm{cal} / \mathrm{mol}-{ }^{\circ} \mathrm{K}$. \\
\hline & res & A work array used in solving matrix equations. Dirmensioning: res(kpar). \\
\hline$P_{g / m l}$ & rho & Density of aqueous solution, $\mathrm{g} / \mathrm{m}$. It is unique to EQ3NR. \\
\hline$-\underline{\alpha}$ & rhs & A negative copy of the array alpha. Dimensioning: rhs(kpar). \\
\hline$J(x)$ & $\mathbf{r j}$ & The $J(x)$ funclion used in Pitzer's equations. \\
\hline \multirow[t]{2}{*}{$J(x)$} & rjp & The $S(x)$ function used in Pilzer's equations. \\
\hline & screwd & Under-rclaxation parameter that bounds delmax. \\
\hline \multirow[t]{2}{*}{$\log Q / K$} & si & $\begin{array}{l}\text { Saturation index of a solid solution end-member component. Dimensioning: } \\
\text { si(iktpar,nxtpar). Usage: si(ik,nx) } i \text { is the saturation index for the ik-th component } \\
\text { of the nx-th solid solution. }\end{array}$ \\
\hline & smp100 & 0 : hundred times the smallest positive real ${ }^{*} 8$ number. \\
\hline $\log Q I K$ & siss & $\begin{array}{l}\text { Array of saluration indices of solid solutions. Dimensioning: siss(nxtpar). Usage: } \\
\text { siss(nx) is the saturation index of the } n x-\text { th solid solution. }\end{array}$ \\
\hline$H_{s r} H_{z r}$ & stor1 & $\begin{array}{l}\text { The } H_{s} \text { or } H_{z r} \text { factors defined in Chapter 9. Dimensioning: stor } 1 \text { (nrstpa). Usage: } \\
\text { stor } 1 \text { (nrs) is the factor for the nrs-th reaction. Note that these are defined in terms } \\
\text { of molalitics in EQ3NR, whereas they are defincd in tenus of moles in EQ6 }\end{array}$ \\
\hline
\end{tabular}

values; $k=$ nalpha(n) for the $n$-th solute species pair for which ${ }^{s_{\lambda j}}$ and $s_{\lambda_{i j}^{\prime}}$ coeffcients arc to be computed.

The eloctron activity function; pe $=-\log a_{e}$.

Array of third onder primitive virial coefficients of Pitzer's equations (values at the current specified temperature). Dimensioning: pmu(nmupar). Usagc: pmu(k) is

Tressure, bars.

Array of second order "stort range" primitive virial cochicients er Pitaer's equations (values at the current specified temperature). Dimensioning: pslam(3,nslpar). Usage: pslam( $n, k)$ is the $n$-th such coefficient for the $k$-th species pair. See bsim, dsim1, dsim2, nsimx, and psim.

Pilzer's cquations (values at the curnent specified temperature). Dimensioning: pslm(3,nslpar). Usage: psim( $n, k)$ is the $n$-th such parameter for the $k$-th species pair. Sec bslm, dsim1, dsimz, nslmx, and pslam.

qbassw Logical nag indicating whelher or not basis switching has taken place.

Logical fag indicating woctber or not there are candidates for aulomatic basis The gas constant, $1.98726 \mathrm{cal} / \mathrm{mol}-^{\circ} \mathrm{K}$.

A work array uscd in solving matrix cquations. Dimeosioning: res(kpar).

Density of aqueous solution. $\mathrm{g} / \mathrm{m}$. It is unique to EQ3NR.

A negative copy of the array alpha. Dimensioning: rhs(kpar).

The $J(x)$ funclion used in Pitzer's equations.

The $N(x)$ function used in Pilzer's equations. si(iktpar,nxtpar). Usage: si(ik,nx) $i$ is the saturation index for the ik-th component of the nx-th solid solution.

of molalitics in EQ3NR, whereas they are defincd in tenms of moles in EQ6 
The nominal upper temperature limit of the data file, ${ }^{\circ} \mathrm{C}$.

tdamin The nominal lower temperature limit of the data file, ${ }^{\circ} \mathrm{C}$.

$C_{T 5, m g / k g} \quad$ tdspkg Total dissolved salts, $\mathrm{mg} / \mathrm{kg}$. This is unique to EQ3NR.

$C_{T \$, m g / L} \quad t d s p l \quad$ Total dissolved salts, $\mathrm{mg} / \mathrm{L}$. This is unique to EQ3NR.

tempe Temperature, ${ }^{\circ} \mathrm{C}$.

$T$

tempk Temperature, ${ }^{\circ} \mathrm{K}$.

tolbt Convergence bound on betamx.

Iołdl Convergence bound on delmax.

tolsat Limit on the absoiute value of the affinity of a mineral of nxed composition for the mineral to be listed as "saturated" on the output file. This is unique to EQ3NR.

tolxat Limit on the absolute value of the affinity of a solid solution for the phase to be listed as "salurated" on the output file. This is unique to EQ3NR.

uactop Character string describing the model selected by the user for computing the activity coefficients of aquecous specics; this is set by the iopgl value read from the input file.

ubasis Array of names of aqueous species that are to be switched into the basis set. Dimensioning: ubasis(nsqpar). Usage: ubasis(ns) is the name of the species to be switched into the basis set in place of the ns-ll basis species.

ubbig The name of the basis species corresponding to the mass balance residual with the most extreme positive value; if none is positive, ubbig is assigned a valuc of 'none!.

ubgamx The name of the species corresponding to the max norm (bgamx) of activity coefficient residual functions.

ubneg The name of the basis species corresponding to the mass balance residual with the most extreme negative value; if none is negative, ubneg is assigned a value of 'none'.

ucode A variable containing the name of the code.

udrxd Holding array used to read in the names of specics appearing in reactions listed on the data file. Dimensioning: udrxd(nsqpa1). Usage: udrxd(n) is the name of the species corresponding to the $\mathbf{n}$-th coefficient in the current reaction as it is writlen on the data file. See cdrsd.

uelam Flag read from the supponting data file indicating whelher or not higher order electrostatic terms are to be used in Piker's enuations:

= 'off Higher order electrostatic terms are not used

$=$ 'on' They ane used

uelem Array of names of chenical elements (their chemical symbols). Dimensioning: uelem(nctpar). Usage: uelem(nc) is the name of the ne-1h element. 
uelemd Hoiding array of names of chemical elements. Dimensioning: uelemd(nctpar). Usage: velemd(n) is the name of the $\mathbf{n}$-th element listed for the current species. See cessd.

pendit The string 'endit.'.

ueqlrn A string containing the release number of the supporting EQLIB library.

ueqlst A string containing the stage number of the supporting EQLIB library.

ugas Array of nanes of gas species. Dimensioning: ugas(ng(par). Usage: ugas(ng) is the nanxe of the ng-th gas species.

ujlype Array of character strings that describe the Jflag oplions. Dimensioning: ujtype(nsqpar).

umemb Array of names of solid solution end-member components appearing on the input filc. Dimensioning: umemb(iktpar,nxtpar). Usage: umemb(ikb,nxb) is the name of the ikb-th component in the nxb-th solid solution whose composition is given on the input file. It is thus input file ordered. It is unique to EQ3NR.

umin Array of name of pure minerats. Dimensioning: umin(nmtpar). Usage: umin(ng) is the name of the nm-th pure mineral.

undms Array of names of data file basis specics appearing on the EQ3NR pickup filc and the EQ6 input file. Dirnensioning: undms(nsqpar). Usage: undms(n) is the name of the n-th such species appearing on ouc of these files.

unone The string 'none'.

unrms Array of names of nun basis species appearing on the EQ3NR pickup file and the EQ6 input file. Dimensioning: unrms(nsqpar). Usage: unrms(n) is the name of the $n$-th such species appearing on onc of these files. If unrms(n) refers to a different species than undms(n), then the former is to be switched into the basis set in place of the latter.

uphasI Array of names of aqueous basis species or mineral, solid solution, or gas species required to complete the definition of an input constraint under the jflag $=17,18,19$, 20, and 21 options. Dimensioning: uphas1(nsqpar). Usage: uphasl(n) is the name of such a species required to complete the constraint for the a-th basis specics appearing on the input file. This array is input file ordered. It is unique to EQ3NR.

uphas2 Array of names of solid solution end-member components required to complete the definition of an input constraint under the jflag $=20$ option. Dimensioning: uphas2(nsquar). Usage: uphas2(n) is the name of such a species required to complete the constraint for the n-th basis species appearing on the input file. This array is input file ordered. It is unique to EQ3NR.

uredox The name of an auxiliary basjs sjecies whiclt is part of an agueous redox couple used to calculate the default redox state of aqueous redox couples. It is unique to EQ3NR.

urelno A string containing the relcase number of the code it is contained it. 
usolb Array of names of solid solution phases whose composition are specified on the input file. Dimensioning: usolb(nxtpar). Usage: usolb(nxb) is lbe name of the nxbth such phase. This aray is input filc ordered. It is unique to EQ3NR.

usolx Array of names of solid solutions. Dimensioning: usolx(nxtpar). Usage: usolx(nx) is the name of the nx-th solid solution.

uspec Array of names of aqueous species. Dimensioning: uspec(ustpar). Usage: uspec(ns) is the name of the ns-th aqueous species.

uspecb Array of names of aqueous basis species listed on the input file. Dimensioning: uspecb(nsb). Usage: uspecb(nsb) is the name of the nsb-th such species. This anay is input tile ordered. It is unique to EQ3NR.

ussnp Array of names of solid solution end members listed on the datal file which fail to correspond to any pure mineral on the same filc. Dimensioning: ussnp(iktpar, nxtpar). Usage: assap $(n, n x)$ is the name of the $n$-th such component in the ax-th solid solution.

ustage A string containing the stage number of the code it is contained in.

utill The title (text) from the input filc. Dimensioning: utitl(ntitpa). Usage; utill(n) is the $n-t h$ line of this title.

utitld The title (text) from the datal file. Dimensioning: utitld(ntitpa). Usage: utilld(n) is the n-th line of this tille.

uxmod The name of a species for which an alter/suppress option is specified on the input file. Dimensioning: uxmod(nxmdpa). Usage: uxmod(n) is the name of the species for the n-th such option specified (see jxmod, kxmod, xlkmod, and nxmod).

uxtype Array of character strings that describe the solid solution activity coefficient models defined by the jsol flag. Dimensioning: uxtype(10). Usage: uxtype (n) is the string which describes the moxtel identified by janl(n).

uxvec1 Array containing the names of the species corresponding to master itcration variables. Dimensioning; uzvec1(kpar). Usage: uzvec1 (kcol) is the species name corresponding to the kcol-th master iteration variable.

vmino Array containing the molar volumes of the pure minerals. Dimensioning: vmino(nmtpar). Usage: vmino (nm) is the molar volume of the nm-th pure mineral.

$V_{8} \quad$ volg

The volume of a perfect gas at $298.15^{\circ} \mathrm{K}$ and 1 bar pressure; $V_{g}=22413.6 \mathrm{~cm}^{3}$. It is unique to EQ3NR.

$W_{w,} w_{i \Psi,} w_{i j \psi} \quad w$

Array of solid solution aclivity coefficient model parameters. Dimensioning: $w(i k t p a r, n x t p a r)$. Usagc: $w(i, n x)$ is the $i$-th parameter for $t h e n x-$ th solid solution. These are computed from the coefficients in the apx array.

weight A work array containing weighting factors for aqueous species used in evaluating balance equalions. Dimensioning: weight(nstpar). Usage: weight (ns) is the factor for the ns-th agueous species. 

$N_{w} \quad$ wfs
$x_{04} \quad$ sbar
$x_{\text {ow }}$
xbarb
$x_{\text {oy }}$
xbarh
Weight fraction of solvent water in aqueous solution.
Array of mole fractions of solid solution end-member components. Dimensioning: xbar(iktpar,nxipar). Usagc: $x b a r(i k, n x)$ is the mole fraction of the ik-th compo- nent of the nx-th solid solution.
Array of mole fractions of solid solution encl-member componen!s read from the in- put file. Dimensioning: xbarb(iktpar,nxtpar). Usage: xbarb(ikb,nxb) is the mole fraction of the ikb-th componeat given for the nxb-lh solid solution listed. This aray is input file ordered. It is unique to EQ3NR.
Amay of mole fractions of solid solution end-member components comesponding to the compositions that maxinuize the saturation indices of the comesponcling phases. Dimensioning: xbarh(iktpar,nxtpar). Usage: $x b a r h(i k, n x)$ is the mole fraction of the lk-th component of the nx-th solid solution which maximizes the saturation in- dex of this phase.

$\log x_{\sigma \psi} \quad \quad \quad x b a r l g$

$\boldsymbol{x}_{\boldsymbol{w}}$

$\log x_{w}$

$I$

$\log K_{E h}$

$\log K_{8}$

$\log K_{\phi}$

xlkm

$\log K_{r}$

xiks

$\log Q / K \quad \quad \quad x i q k$

$\log Q / K \quad \mathbf{x q} \mathbf{k x}$

Array of log mole fractions of solid solution end-member components. Dimensioning: xbarjg(iktpar,nxtpar). Usage: $x$ barlg(ik,nx) is the log mole fraction of the ikth component of the nx-th solid solution.

The mole fraction of solvent water.

The log mole fraction of solvent water.

Ionic strength, molal.

Log equilibrium constant of the half seaclion relating the hypothetical electron and $\mathrm{O}_{2(g)}$

Array of log cquilibrium constants of the dissolution reactions of gas species. Dimensioning: $x$ lkg(ngtpar). Usage: $x l k g(a g)$ is the log cquilibrium constant for the ng-th gas.

Array of $\log$ equilibrium constants of the dissolution reactions of pune minerals. Dimcnsioning: $x$ lkm(nmlpar). Usagc: $x$ lkm(nm) is the log equilibrium constant for the nm-th pure mineral.

xlkmod The $\log K$ alter option parameter for an alter/suppress option specified on the input file. Dimensioning: xlkmod(nxmdpa). Usage: $x \mathrm{lkmod}(n)$ is the parameter for the n-th such option specified (sce jxmod, kxmod, uxmod, and nxmod).

Array of log equilibrium constants of the dissociation/destruction reactions of aqueous species. Dimensioning: xlks(nrstpa). Usage: xlks(nrs) is the log equilibrium constant for the nrs-th such species.

Amay of saturation indices of pure minerals. Dimensioning: xlqk(nmtpar). Usage: xlqk(nm) is the saturation index for the nm-th pure mineral.

Array of saturation indices of solid solution end-member components. Dimensioning: $x q k x$ (iktpar,nxtpar). Usage: $x q k x(i k, n x)$ is the saturation index for the ik-th component of the nx-th solid solution. 
Array of electrical charges of the aqueous species. Dimensioning: $\mathbf{z}$ (nstpar). Usage: $z$ (ns) is the electrical charge of the ns-th aqueous species.

$\frac{z_{i}^{2}}{2}$

zaq2 Array of values equal to one balf the charge squared of the aqueous species. Dimensioning: zsq2(nstpar). Usige: $\mathbf{z s q 2 ( n s )}$ is one half the charge squared for the ns-th aqueous specics.

$z$

2velg1 Array of logarithmic master iteration variables. Dimensioning: 2velg1(kpar). Us-

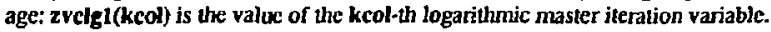




\section{Appendix B. Glossary of EQ3NR Modules}

EQ3NR is a moderately large code. The source code consists of the main program and a number of subroutines. In addition, EQ3NR uses a number of modules from the EQLIB library. These are described in Appendix B of the EQ3/6 Package Overview and Installation Guide (Wolery, 1992). The modules are described as " $r$ " files, as this is how they are normally worked with under a UNIX operating system. For a description of the code architecture, see Chapter 10.

arrsel.f This modulc is calied by eq3ec.f. It sets up the matrix structure for Newton-Rapbson iteration and estimates starting yalues for the iteration variables. The initial estimates are refined according to a preNewion-Raphson oplimization algorithm. They may also be relned by automatic basis switching, if this feature is enabled.

arrsim.f This module is called by arrset.f to compute those basis species concentralions which by the nature of the chosen constraints must be estimated simultanously.

balcon.f This module is called by matrix.f. It writes the part of the Jacobian matrix that consists of rows corresponding to balance equations (mass and charge balance).

betas.f This module is called by arrset.f and the EQLIB modulcs newton.f and nrstep.f (these latter two modules call it as betae). It compt'cs the residual functions $\left(\boldsymbol{\alpha}, \boldsymbol{\beta}\right.$, and $\beta_{\max }$ ).

dawfix.f This module is called by arrsim.f. It determines whether or not the problem input constraints directly fix the activity of water, as for example would happen if one constrained dissolved calcitum by cquilibrium with gypsum and dissolved sulfate by cquilibrium with anhydrite.

echox.f This module is called by eq 3nr.f. It writes a description ot the input problem on the output fite. Unlike the "instant echo" of the input file, this description includes data file statistics, any default values that were chosen by EQ3NR, and any changes in the inpul constraint options made by the code to resolve inconsistencies among thesc constraints or with truncation limits imposed on certain run parameters.

eq3nrif This is the main program of EQ3NR. It supervises the speciation-solubility calculation. It connects the necessary files (input, data1, oulput, pickup, and rlist). It jnitializes key arrays and sets default values for imporant nu parameters. It also does an extensive checking of the problem that has been input, looking for inconsistencies and missing inputs that are required by the options ehosen.

figstx.J This module is called by erg $3 \mathrm{nr}, \mathrm{f}$. It sets up the s]ecics status switch arrays jsfiag, jmflag, jkflag. jxflag and jgilag.

gases.I This module is called by seripx.f. It computes the eguilibrium fugacities of gas species.

gesp.f The module is called by indatx,f. It interprets the constraints (jflag, csp) for basis species listed on the input file by matching the species names with those read from the supporting data file.

getrdx.f This module is called by rityp4.f. It gets the redox constraints specificd on the input file in " $D$ " fornlat.

getspe.f This module is ealled by both getrdx.f and $r$ dtyl44.f. It gets input constraints for the basis species Jisted on the inpul file in "D" lormat.

getss.f This module is called by both getspc.f and rdtyp5.f. It gets solid solution data that are part of constraints listed on the inpul file in " $D$ " fomat. 


\begin{abstract}
indatx.f This module is called by eq3ar.f. It reads the data file data1.
init3v.f This module is called by rdninp.f.

matrix.f This module is called by the EQLIB routinc arstep. $f$ (which calls it by the name of matrxe). It computes the Jacobian matrix $(J$.
\end{abstract}

ncmpx.f This module is called by arrset.f and the EQLIB modules ugcadv.f and nrstep.f (which call it by the name of nempe). It computes all parameters that derive from the primary iteration variables and are necessary to writc the Jacobian matrix (c.g., all aqueous species concentrations and activities).

ndiagx.f This module is called by eq3nr.f. If Newton-Rapbson iteration fails, it attempts to generate diagnostics.

rd3ts.f This module is called b; rdninp.f. 11 gets the input for total dissolved salts from the input file in "D" format.

rdinp.f This module is called by eq3nr.f. $1 t$ oversees reading the input file. If the input file is in " $W$ " fomat, it calls readx. $f$ to carry out this function. If the input filc is in " $D$ " format, it calls rdninp. f to do this.

rdninp.f This modulc is called by rdinp.f. It carries out reading the inpul file in "D" format.

rdtypl.f This module is called by rdninp.f. It gets the input for temperalure and density fron the input file in "D" fomat.

rdtyp2,f This module is called by rdninp.f. It gets the input for electrical balancing from the input file in " $D$ " format.

rdtyp4.f This module is called by rdninp.f. It gets the input for the basis species constraints from the input file in " $D$ " fomat. It does this by calling getrdx.f and getspe.f.

rdlyp5.f This module is called by rdninp.f. It gets the input for solid solutions from the input file in " $D$ " format. It calls getss.f.

rdtyp9.f This module is called by rdninp.f. It gets the input for tolbt, toldl, toksat, and itermx from the input file in " $D$ " formal.

readx.f This module reads the input file in "W" format. It writcs an "instant echo" of this input data on the output file. It contains full intemal documentation.

scribo.f This module is called by eqJnr.f. It writes the pickup file in "W" format.

scribx.f This module is called by eq3nrf. It writes the pickup file in " $D$ " format.

scripx.f This module is called by eq3nr.f. This module writes the results of the speciation-solubility calculation on the output filc.

setup.I This module is called by eq3nr.f. This module converts input concentration dala other than molalities to molalities. 


\section{Appendix C. EQ3NR Error Messages}

All EQ3/6 error messages fit into one of three categories: error, warning, and note. An error implies a fatal error. Execution of the current input problem will cease without completion, immediately in some cases, later in others. Which is the case depends on whether it makes more sense to stop immediately or to continue checking for other errors before ceasing execution. A warning indicates a condition which may or may not represent a real error. A note indicates a condition which could possibly indicate an error, but normally does not. All three types of messages are written to both the screen file and the output file. If an error message is issued, analysis of the problem may be facilitated by checking any preceding error, warning, or note messages.

Each EQ3/6 error message has the following format:

* msgtype - (sourcelmodule) Message.

where msgtype $=$ error, warning, or note, source is the root name of the source file (e.g., eqlib, eqpt, eq3n), or eq6) containing the module, module is the name of the module (main program or subroutine) which writes the message, and Message is the message itself. The messages are designed to be as self-explanatory as possible. The messages are reproduced here using $A A A A$ to stand for a character variable, $I I I$ for an integer, and $R K R R$ for a floating point number.

Most of the error messages that users are likely to encounter deal with problems regarding the input file, the data file, or both of these. In most instances, the meaning of these messages should be immediately ciear to the user. In other instances, it may be necessary to search out other information. In such cases, there are three principal actions that users should take. The first is to check the output file for additional diagnostic messages (warnings and notes) which may bear on the matter. If this does not suffice to identify corrective action, compare the instant echo of the input fle on the output file with the original input file. You may find that certain data were not entered in the correct fields, that certain inputs fail to correspond with the necessary lines to follow, or that a line is missing or you have an extra line. In addition, it may help to re-run the problem with the debugging option switch iodb1 set to 1 or 2 . This will trigger the printing of additional information which should help to identify the problem. A small number of messages deal with installation errors. These should also be quite clear.

Some messages deal with programming enrors. The user should see these rarely if ever. These are likely to appear somewhat more cryptic to users. Problems of this type must be dealt with by diagnosing the problem (probably with the help of a symbolic debugger) and modifying the code. Most users should probably not attempt corrective action of this sort. The code custodian should be notified of suspected programming errors and may be able to provide fixes.

Some of the messages displayed in this appendix are followed by Comments that may help to explain them. Users of EQ3NR may also encounter error messages from EQLIB modules. These messages are listed in similar format in the EQ3/6 Package Overview and Installation Guide (Wolery, 1992). The errors are listed first, then the warnings and finally the notes.

Message: * crror - (ç3nr/arrset) Have encountered an automatic basis switcling error in loop LIII.

Commen: This is a programming error. 
Message: * error - (eq3nr/arrsim) The phase assemblage by the specified solubility constraints fixes the activity of water. The code is presently unable to solve problems of this type.

Message: * enor - (eg3nr/arrsim) The specified counterion "AAAA" in the jflag = 17 or 18 option for "AAAA" is not in the basis set.

Message: * error - (cq3nr/arrsim) The speciation model appears to be singular. There is probably a violation of the mineralogic phase rule in the set of solubility constraints specified on the input file.

Message: * error - (cq3nr/betas) Have encountered an illegal jflag valuc = IIII for the species "AAAA".

Message: * error - (eq3uy/eq3nr) Can not use the jllag= IIII option for "AAAA" because this species is suppressed.

Message: * error - (eq3nr/eq3nr) Can not use the jflag= IIII option for "AAAA" because this species has no charge.

Message: " eiror - (eq3nr/en3nr) Can not use the jllag= IIII option for "AAAA" because the specified counterion "AAAA" is not in the aclive basis set.

Message: * error - (cq3nr/eq3nr) Can not use the jflag= IIII option for "AAAA" because the specified counterion "AAAA" is not in the present system.

Message: * error - (eq3nr/eq3nr) Can not use the jllag= IIII option for "AAAA" because the specified counterion "AAAA" is suppressed.

Message: * error - (cq3nr/eq3n) Can not use the jlag= Ill option for "AAAA" because the specified counterion "AAAA" has zero charge.

Message: * error - (eq3ar/eq3nr) Can not use the jlag= IIII option for "AAAA" because the specified counterion "AAAA" has the same charge sign.

Message: * error - (eq3ar/eq3ar) Choosing iopt $1=-3$ requires setting jflag(o2(g)) to 19,20 , or 21 . The input file has jHag(02(g)) = IIII.

Message: * error - (eq3nr/eq3nr) The species "AAAA" was selected to determine the redox state, but the associated reaction is not a redox reaction.

Message: * error - (eq3ar/eq3nr) The specics "AAAA" was selected to determine the redox state, but it is not in the present system.

Message: * error - (eq3ar/eq3ar) The species "AAAA" was selected to specify the redox state, but it has a jlag value of III. The jflag for such species can not have a value of 27 or 30 .

Comment: You have to enter some actual data for this species. The jflag $=27$ and 30 options do not correspond to inpus of aciual dala.

Message: * error - (cq3n/eq3nr) The species "AAAA" was selected to specify the redox state, but the necessary corresponding strict basis species representing the complementary oxidation state is not in the present systen.

Comment: To use this option for the redor state, you must enter data for both the auxiliary basis species whose associated reaction defines the couple, and the sirict basis species which represents the other half of the redox couple and appears in that reaction. 
Mescage: * error - (eq3nr/eq3nr) Have illegal negative csp value of RRRR for the basis species "AAAA".

Message: * error - (eq3nr/eq3nr) Did you mean to specify alkalinity for "AAAA"? Because of theoretical problems with the concept and not uncommon analytical difficultics, EQ3/6 no longer allows the use of alkalinity.

Comment: If you have not already done so, read she section on alkalinity in Chapter 2.

Message: * error - (eq3nr/eq3ar) The species "AAAA" can not be constrained by the reaction-

Comment: This is followed by the reaction in question.

Message: * error - (eq3ar/eq3nr) The species "AAAA" can not be constrained by equilibrium with a solid solution because iopt $4=$ IIII, not 2.

Comment: In order to use such a constrain, it is necessary 10 inpur the composition of the solid solution.

Message: * error - (cq3ur/eq3nr) The species "AAAA" has a jllag value of mII. This is not permitted. This species is in the strict basis set and therefore has no associated reaction in which it is destroyed.

Message: * епог - (eq3nr/eq3nr) The following heterogeneous riaclion constraint has been used more than once-

Comment: This is followed by the reaction in quession.

Message: * error - (eq3nr/eq3nr) The input fle bas jflag(o2(g) = IIII. This requires ioptl to be -3 , but ioptl is now set to IIII.

Message: * error - (eq3nr/eq3nr) Hybrid newton-raphson iteration failed after IIII steps. Calling diagnostics routine.

Message: * error- (eq3nr/gcsp) The basis species "AAAA" was specified more than once on the input file.

Message: * error - (eq3nr/getrdx) When specifying redox parameters, column four must contain "eh", "pe", "fo2lg" or 'tedox couple". Check INPUT filc line: IIII

Message: * crrr - (eq3ns/getrdx) Brcak line or end-of-file found where redox couple input was expected. Check INPUT file linc: IIII

Message: * eло - (eq3nr/getspc) Species was not specified in column 1. Cherk INPUT file line: III

Message: * error - (eq3nr/getspc) Maximum number of allowed enlries: IIII. Have excceded this value on INPUT file line HII

Message: * error - (cq3nt/getspc) The "pH" species can only be " $\mathrm{b}+$ ". Check INPUT file line: IIII

Message: * error - (cq3nt/getspc) The "pHCl" species cau only be ' $\mathrm{h}+$ ". Check INPUT file line: IIII

Message: * error - (eq3n/getspc) Colunn 2 can not be blank for the "gas", "mineral", "log activity combo". and "log mean activity" constrailss. Check INPUT file like: IJII

Message: * error - (cq3nr/getspc) Column 2 must left blank for the "log activity", "ph", and "phcl" constraints. Check INPUT file line: IIII

Message: * error - (ę3nz/getss) Maximum number of allowed entries: IlII. Have exceeded this value on INPUT file line: IIII 
Message: * error - (eq3nu/getss) Must specify all end-members of a solid solution (mole fractions must sum to 1.0). Check above INPUT file line: IIII

Message: * error - (eq3ar/getss) Solid solution name can not be blank. Check INPUT file line: IIII

Message: * error - (eq3ar/getss) End-member names can not b blank. Check INPUT file line: IlII

Message: * error - (eq3n/getss) Maximum number of end-members per solid solution: IIII. Check above INPUT file line: IIII

Message: * error - (cq3n/indatx) Have wrong file beader = "AAAA" on the data file. The first five characters must be "datal".

Comment: Make sure you have nol defined datal to be a data0 file.

Message: * enor - (eq3ar/indatx) The number of chemical elements on the data fle is III. This excceds tie dimcnsioncd limit (nstparj) of IIII.

Message: * enror - (eq3nr/indatx) The number of basis species on the data file is IIn. This exceeds the dimensioned limit (nsqpar) of IIII.

Message: * error - (eq3nr/indatx) The composition of species "AAAA" on the data file is described in temus of an unrecognized chemical clement called "AAAA". If this is an actual element, it is not in the list of chemical elements on this data filc.

Message: * error - (cq3nr/indatx) The maximum number of aqueous species (nstpar) is llII. This bas been exceeded while trying to read the data filc.

Message: * error - (eq3nr/indatx) The reaction for the desinuction of the species "AAAA" is written on the data file in terms of an unrecognized basis species called "AAAA". If this is an actual species, it is not among the basis species on this data fite.

Message: * error - (eq3nr/indatx) The maximum number of fure minerals (nmtpar) is IIll. This has been exceeded while trying to read the data file.

Message: * eлror - (eq3n/indalx) The maximum number of gas sfexies (ngtpar) is IIII. This has been exceeded while trying to read the data file.

Message: * error - (eq3arfindatx) The basis species "AAAA" was referenced on the input file but was not read from the data file.

Comment: The name of the basis species may contain a rypographical error. Othenvise, the data file does not contain this species. You may be using the wrong data file.

Message: * error - (eq3nr/indatx) The maximum number of solid solutions (nxtpar) is III. This has been exceeded while trying to read the dala file.

Messagt: * ertor - (en3nr/indatx) The specits "AAAA" is specilied in uscr-directed basis switcles to switch with both "AAAA" and "AAAA".

Message: * error - (eņ3nrfindatx) The species "AAAA" is specilied in a user-directed basis switch to switch with "AAAA", but is not in the list of aquicous species read from the data file. 
Message: " error - (eq3nr/indatx) The species "AAAA" is required for an abs(zj)*log ai +/-abs(zi)*log aj constraint but was not read from the data file.

Message: * error - (eq3nr/indatx) The specics "AAAA" is required for a log a(t/-,ij) constraint but was not read from the data file.

Comment: "a(+/-,ij)" denotes the mean activity of ions $i$ and $j$.

Message: * crtor - (eq3nr/indatx) The species "AAAA" is required for a heterogeneous equilibrium constraint but was not read from the data file.

Message: * cror - (cq3nr/matrix $)$ Have encountered an illcgal jllag value $=111 \mathrm{l}$ for the species "AAAA".

Comment: This is a programmer error.

Message: * error - (eq3nr/rdninp) Looking for heading: "AAAA" Found string: "AAAA" Sec INPUT fi]e line: IIII

Message: * error - (eq3ns/rdninp) End-of-file unexpectedly cocountered. See INPUT file linc: III

Message: * error - (cq3nr/ndninp) Enors encountered while reading the INPUT file. Process stopped

Message: * error - (eq3nr/rdninp) The electrical balancing species "AAAA" was not found among the basis species listed on the input file.

Message: * error - (eq3nr/roninp) The species " $h+"$ must be specificd in the species list on the input file.

Message: * error - (eq3nr/rdtyp2) Only one electrical balancing option can be selected. Check INPUT file line: IIII

Message: * error - (eq3nr/rdtyp9) Unrecognized tolesance variable "AAAA". Check INPUT file line: IIII

Message: * error - (eq3nI/rityp9) Invalid tolerance descriptor string "AAAA". Check INPUT file line: IIII

Message: * error - (eq3nr/readx) The input file has no tille.

Message: * error - (eq3nr/readx) The maxirnum number of nxmod options (nxmdpa) is IIII. This has been exceeded while trying to read the input file.

Message: * crror - (eq3nr/readx) The maximum number of basis species (nsqpar) is IIIl. This bas been exceeded while trying to read the data filc.

Message: * crror - (eq3nz/scribo) Bad pickup file has been written. The basis species "AAAA" appears on it but is not in the model.

Commen: The pickup file contains a strict basis species that is not in the model. You used an auxiliary basis species which is linked to this strict basis species. The EQ3NR calculation is still valid, but you can not use the pickup file to input the solution model to EQ6. If you want to do this, re-run the problem switching the auxiliary basis species in question into the strict basis ser.

Message: * error (eq3nt/setuj)) An undefined jflag yalue of IIII was specificd for the basis species "AAAA".

Message: * warning - (e(p) 3nr/cq3nr) Tlx input file contains conflicting redox options - iopt $=$ IIII overrides jlla$g(02(g))=$ I]II. 
Message: * warning - (eq3nr/eq3nr) The input file contains conflicting redox options - ioptl = III overrides uredox = "AAAA".

Message: * waming - (eq3nr/eq3tur) The species "AAAA" was specified to be adjusted for electrical balance, but it is not in the set of basis species.

Message: * warning - (eq3nr/eq3nr) The specics "AAAA" was specified to be adjusted for electrical balance, but it has no electrical charge. Success will depend on its concentration affecting that of one or more charged species with which it is in equilibrium.

Message: * warning - (eq3nr/eq3nr) The species "AAAA" was specified to be adjusted for electrical balance, but it is not in the present model.

Message: * waming - (eq3nr/eq3nr) Tried to pick a species to be adjusted for electrical balance, but did not succeed.

Message: * waming - (eq3nr/eq3nr) The species "AAAA" was specified to deflne the default redox state, but is not in the active auxiliary basis set.

Message: * waroing - (eq3nr/eq3nr) The strict basis species "AAAA" has an illegal jllag value of 1111.

Message: * waraing - (eq3ur/rcadx) The input line for $02(\mathrm{~g})$ will be ignored because ioptl is not set $10-3$.

Message: * note - (eq3nranrset) Can not switch the species "AAAA" out of the basis because it is tied up in a jlag $=17$ or 18 option for another species.

Message: * note - (eq3nt/anset) Optimization ended outsidẹ requested linits.

Comment: Don't panic. Hybrid Newton-Raphson iteration may still succeed. If so, the results of the run are still perfectly good If it doesn't succeed, see the comment following the following message.

Message: * note - (eg3nt/anset) Optimization ended far outside requested limits.

Comment: Don't panic. Hybrid Netwon-Raphson iteration may still succeed, though the probability of this is significuntly diminished. If it does succeed, the results of the run are still perfectly good. If it doesn't succeed, re-run with iodb set to 1 or 2 in order to see what is going on during the optimization stage. You may be able to get the problem to converge by directing the code ro make certain basis switches.

Message: * note - (eq3n/arrsim) The matrix solver routine (eqlib/msolvr) failed.

Message: * note - (eq3nr/artsim) The species "AAAA" has a required concentration near RRRR molal.

Comment: Don't panic. This is not always fatal. If the code fails fo solve the problem, however, this probably means that one or more equilibrium constraints ( $\mathrm{j}$ fling $=19,20,21$, or 27) are such that no sensible answer exists to the problem currently posed. Think about what you are asking the code to do.

Message: * note - (eq3ny/echox) The species "AAAA" is not in the model.

Message: * note- (eg 3 mr/en3ur) The input value for the iopr9 print option llag is inconsistent with the s|ccified value of iopg 1 (choice of model for activity coefficients of aqueous species). The iopr 9 flag has been reset to 0 .

Message: * note - (ç3ur/eq3nr) Have picked the species "AAAA" to be adjusted for electrical balance. Any other specified constraint will be overridden. 
Message: ₹ note - (eq3nr/eq3nr) Could not compute acceptable starting estimates for Newton-Raphson iteration. Will therefore not attempt it.

Comment: Re-run with iodb set 101 or 2 in order ro see what is going on during the optimizotion stage. You may be able to ger the problem to converge by directing the code to make cerlain basis switches.

Message: * note - (eq3nr/eq3nr) Can not write a valid pickup file for the current problem because the auxiliary basis species in each of the following strict-auxiliary basis species pair(s) is present in the model while the corresponding strict basis species is not. Add a trivial amount of each such strict basis species to the model, or switch it from the strict set to the auxiliary set and suppress it if necessary.

Comment: The relevant data follow this message. The pickup fle contains a strict basis species that is not in the model. You used an auxiliary basis species which is linked so this strict basis species. The EQ3NR calculatlon is still valid, but you can not use the pickup file to input the solution model to EQ6. If you want to do this, re-run the problem switching the auxiliary basis species in question into the strict basis set.

Message: * note - (eq3ar/getspc) A non-zero concentration should not be specified as an input parameter for a "mineral", "solid solution", "dependent", or "eliminated" species. Chock INPUT file line: III

Message: * note - (eq3ur/ndiagx) The ion being adjusted to achieve electrical balance is crasbing to zero. Electrical balaucing most be done on an ion of opposite charge.

Message: * note - (eq3nr/ndiagx) The oxygen fugacity is crasling, probably because a bad electrical balance constraint on $h+$ is causing the concentration of that species to crash to zero.

Message: * note - (eq3ar/ndiagx) The oxygen fugacity is crashing, probably because of a bad constraint on one of the aqueous species appearing in the redox reaction that is being used to constrain the redox state.

Message: * note - (eq3nr/ndiagx) No diagnostics were generated from the failed iteration. Look at the del and beta data in the iteration summary for clues to why it did not work. 


\section{Appendix D. Notes on Known Bugs and Such}

This appendix presents notes on known bugs and other known unusual phenomena concerning EQ3NR.

1. The code tends to converge slowly or not at all in dealing with extremely concentrated electrolyte solutions. Roughly speaking, these are solutions whose ionic strengths are greater than about 12 molal. However, there is no simple way to accurately categorize the code's performance envelope.

For a complete list of known bugs and such for EQ3/6, see Appendix D of the EQ3/6 Package Overview and Installation Guide (Wolery, 1992). 